\title{
BTS guideline for oxygen use in adults in healthcare and emergency settings
}

\author{
B R O'Driscoll, ${ }^{1,2}$ L S Howard, ${ }^{3}$ J Earis, ${ }^{4}$ V Mak, ${ }^{5}$ on behalf of the British Thoracic \\ Society Emergency Oxygen Guideline Group
}

- Additional material is published online only. To view please visit the journal online (http://dx.doi.org/10.1136/ thoraxjnl-2016-209729).

${ }^{1}$ Respiratory Medicine, Salford Royal Foundation NHS Trust, Salford, UK

${ }^{2}$ Manchester Academic Health Sciences Centre (MAHSC), Manchester, UK

${ }^{3}$ Hammersmith Hospital, Imperial College Healthcare NHS Trust, London, UK ${ }^{4}$ University of Liverpool, Liverpool, UK

${ }^{5}$ Imperial College Healthcare NHS Trust, London, UK

Correspondence to Dr BR O'Driscoll, Respiratory Medicine, Level 4 Brooke Building (Orange Area), Salford Royal Foundation NHS Trust, Stott Lane, Salford M6 8HD, UK; ronan.0@driscoll@srft.nhs.

Received 15 November 2016 Revised 3 February 2017 Accepted 12 February 2017

\section{SLinked}

- http://dx.doi.org/10.1136/ thoraxjnl-2017-209951

- http://dx.doi.org/10.1136/

bmjresp-2016-000170

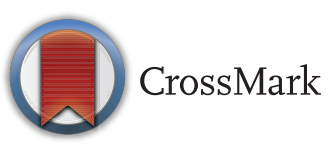

To cite: O'Driscoll BR, Howard LS, Earis J, et al. Thorax 2017:72:i1-i90.

\section{EXECUTIVE SUMMARY OF THE GUIDELINE}

Philosophy of the guideline

- Oxygen is a treatment for hypoxaemia, not breathlessness. Oxygen has not been proven to have any consistent effect on the sensation of breathlessness in non-hypoxaemic patients.

- The essence of this guideline can be summarised simply as a requirement for oxygen to be prescribed according to a target saturation range and for those who administer oxygen therapy to monitor the patient and keep within the target saturation range.

- The guideline recommends aiming to achieve normal or near-normal oxygen saturation for all acutely ill patients apart from those at risk of hypercapnic respiratory failure or those receiving terminal palliative care.

\section{Assessing patients}

- For critically ill patients, high-concentration oxygen should be administered immediately (table 1 and figure 1 (chart 1)) and this should be recorded afterwards in the patient's health record.

- Clinicians must bear in mind that supplemental oxygen is given to improve oxygenation but it does not treat the underlying causes of hypoxaemia which must be diagnosed and treated as a matter of urgency.

- The oxygen saturation should be checked by pulse oximetry in all breathless and acutely ill patients, 'the fifth vital sign' (supplemented by blood gases when necessary) and the inspired oxygen concentration should be recorded on the observation chart with the oximetry result. (The other vital signs are pulse rate, blood pressure, temperature and respiratory rate).

- Pulse oximetry must be available in all locations where emergency oxygen is used. Clinical assessment is recommended if the saturation falls by $\geq 3 \%$ or below the target range for the patient.

- All critically ill patients outside of a critical care area (eg, intensive care unit (ICU), high dependency unit (HDU), respiratory HDU) should be assessed and monitored using a recognised physiological track and trigger system such as the National Early Warning Score (NEWS).

\section{Target oxygen prescription}

- Oxygen should be prescribed to achieve a target saturation of $94-98 \%$ for most acutely ill patients or $88-92 \%$ or patient-specific target range for those at risk of hypercapnic respiratory failure (tables 1-4).

- Best practice is to prescribe a target range for all hospital patients at the time of admission so that appropriate oxygen therapy can be started in the event of unexpected clinical deterioration with hypoxaemia and also to ensure that the oximetry section of the early warning score (EWS) can be scored appropriately.

- The target saturation should be written (or ringed) on the drug chart or entered in an electronic prescribing system (guidance on figure 1 (chart 1)).

\section{Oxygen administration}

- Oxygen should be administered by staff who are trained in oxygen administration.

- These staff should use appropriate devices and flow rates in order to achieve the target saturation range (figure 2 (chart 2)).

- Staff should be trained in the use of a range of different oxygen delivery devices to ensure oxygen is delivered safely.

\section{Monitoring and maintenance of target saturation}

- Oxygen saturation and delivery system (including flow rate) should be recorded on the patient's monitoring chart.

- Oxygen delivery devices and flow rates should be adjusted to keep the oxygen saturation in the target range. Prompt clinical assessment is required if oxygen therapy needs to be initiated or increased due to a falling saturation level.

- Oxygen should be prescribed and a signature should be entered on the drug chart on each drug round.

\section{Weaning and discontinuation of oxygen therapy}

- Oxygen should be reduced in stable patients with satisfactory oxygen saturation.

- Oxygen should be discontinued once the patient can maintain saturation within or above the target range breathing air but the prescription for a target range should be left in place in case of future deterioration and to guide EWS/ NEWS.

\section{SUMMARY OF GUIDELINE RECOMMENDATIONS AND GOOD PRACTICE POINTS}

A Achieving desirable oxygen saturation ranges in acute illness (see sections 6 and 8 and figures 1-2)

A1: This guideline recommends aiming to achieve a normal or near-normal oxygen saturation for all 
Table 1 Critical illness requiring high levels of supplemental oxygen
Section 8.10
The initial oxygen therapy is a reservoir mask at $15 \mathrm{~L} / \mathrm{min}$ pending the availability of reliable oximetry readings.
For patients with spontaneous circulation and a reliable oximetry reading, it may quickly become possible to reduce the oxygen dose while maintaining a target saturation range of $94-98 \%$.
If oximetry is unavailable, continue to use a reservoir mask until definitive treatment is available.
Patients with COPD and other risk factors for hypercapnia who develop critical illness should have the same initial target saturations as other critically ill patients pending the results of blood gas results after which these patients may need controlled oxygen therapy with target range $88-92 \%$ or supported ventilation if there is severe hypoxaemia and/or hypercapnia with respiratory acidosis.

\begin{tabular}{lll}
\hline & Additional comments & \multicolumn{1}{c}{ Recommendations } \\
\hline Cardiac arrest or resuscitation & $\begin{array}{l}\text { Refer to resuscitation guidelines for choice of delivery device during active resuscitation. } \\
\text { Give highest possible inspired oxygen concentration during CPR until spontaneous } \\
\text { circulation has been restored. }\end{array}$ & Recommendation E1 \\
$\begin{array}{l}\text { Shock, sepsis, major trauma, drowning, anaphylaxis, } \\
\text { major pulmonary haemorrhage, status epilepticus }\end{array}$ & Also give specific treatment for the underlying condition & Recommendations \\
Major head injury & Early tracheal intubation and ventilation if comatose & \multicolumn{1}{c}{ Recommendation E5 } \\
Carbon monoxide poisoning & $\begin{array}{l}\text { Give as much oxygen as possible using a bag-valve mask or reservoir mask. Check } \\
\text { carboxyhaemoglobin levels. } \\
\text { A normal or high oximetry reading should be disregarded because saturation monitors } \\
\text { cannot differentiate between carboxyhaemoglobin and oxyhaemoglobin, owing to their } \\
\text { similar absorbances. } \\
\text { The blood gas PO2 will also be normal in these cases (despite the presence of tissue } \\
\text { hypoxia). }\end{array}$ \\
\hline
\end{tabular}

COPD, chronic obstructive pulmonary disease; $\mathrm{CPR}$, cardiopulmonary resuscitation; $\mathrm{PO}_{2}$, oxygen tension arterial or arterialised blood gases.

acutely ill patients apart from those at risk of hypercapnic respiratory failure (grade D).

A2: The recommended target saturation range for acutely ill patients not at risk of hypercapnic respiratory failure is $94-98 \%$ (grade D).

A3: For most patients with known chronic obstructive pulmonary disease (COPD) or other known risk factors for hypercapnic respiratory failure (eg, morbid obesity, cystic fibrosis (CF), chest wall deformities or neuromuscular disorders or fixed airflow obstruction associated with bronchiectasis), a target saturation range of $88-92 \%$ is suggested pending the availability of blood gas results (grade A for COPD, grade D for other conditions).

A4: Most non-hypoxaemic breathless patients do not benefit from oxygen therapy, but a sudden reduction of $\geq 3 \%$ in a patient's oxygen saturation within the target saturation range should prompt fuller assessment of the patient (and the oximeter signal) because this may be the first evidence of an acute illness (grade D).

A5: Since oxygenation is reduced in the supine position, fully conscious hypoxaemic patients should ideally be allowed to maintain the most upright posture possible (or the most comfortable posture for the patient) unless there are good reasons to immobilise the patient (eg, skeletal or spinal trauma) (grade D).

\section{B Clinical and laboratory assessment of hypoxaemia and hypercapnia}

B1: Fully trained clinicians should assess all acutely ill patients by measuring respiratory rate, pulse rate, blood pressure and temperature and assessing circulating blood volume and anaemia (see section 7). Expert assistance from specialists in intensive care or from other disciplines should be sought at an early stage if patients are thought to have major life-threatening illnesses and clinicians should be prepared to call for assistance when necessary including a call for a 999 ambulance in prehospital care or a call for the resuscitation team or ICU outreach team in hospital care (grade D).
B2: Oxygen saturation, 'the fifth vital sign', should be checked by trained staff using pulse oximetry in all breathless and acutely ill patients (supplemented by blood gases when necessary) and the inspired oxygen device and flow rate should be recorded on the observation chart with the oximetry result (grade D).

B3: Initial clinical assessment and subsequent monitoring of acutely unwell patients should include the use of a recognised physiological 'track and trigger' system, such as the NEWS which may trigger clinical review due to hypoxaemia, need for supplementary oxygen or for other reasons (grade D).

B4: For patients who are at risk of hypercapnic respiratory failure, it is recommended that the relevant section of the 2017 NEWS chart should be used. Points are awarded if the oxygen saturation is below or above the target range (grade D).

Good practice points for clinical assessment of patients with suspected hypoxaemia

$\checkmark$ The medical history should be taken when possible in an acutely breathless patient and may point to the diagnosis of a particular acute illness such as pneumonia or pulmonary embolism or an exacerbation of a chronic condition such as COPD, asthma or heart failure.

$\checkmark$ Never discontinue oxygen therapy to obtain an oximetry measurement on room air in patients who clearly require oxygen therapy.

$\checkmark$ Physical examination should be undertaken urgently. This may provide evidence of a specific diagnosis such as heart failure or a large pleural effusion, but it is common for the cause of breathlessness to remain undiagnosed until the results of tests such as chest radiographs are available.

$\checkmark$ Record arterial oxygen saturation measured by pulse oximetry $\left(\mathrm{SpO}_{2}\right)$ and consider blood gas assessment in patients with unexplained confusion and agitation as this may be presenting feature of hypoxaemia and/or hypercapnia (cyanosis is a difficult physical sign to record confidently, especially in poor light or with an anaemic or plethoric patient). 


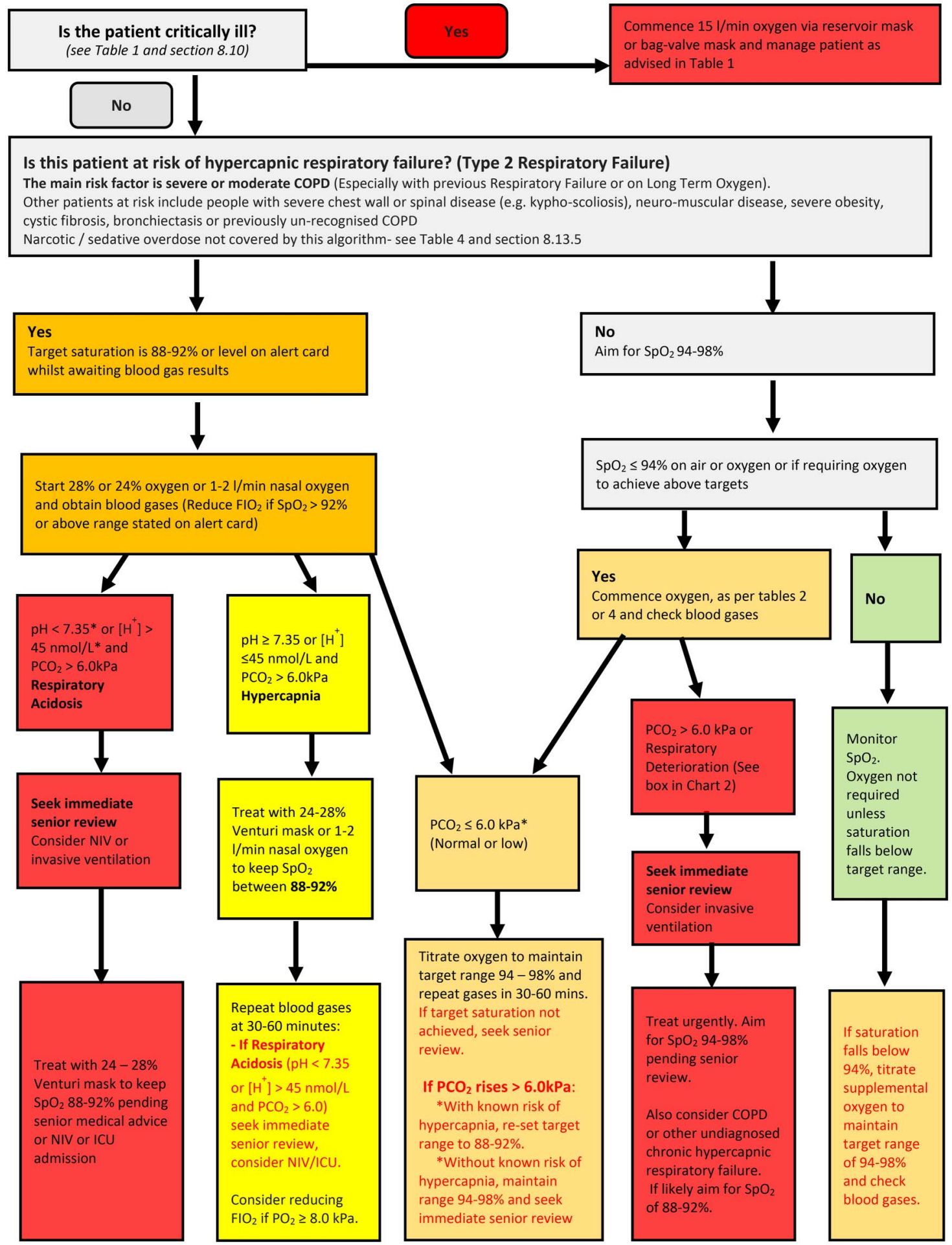

Figure 1 Chart 1 - Oxygen prescription for acutely hypoxaemic patients in hospital. Any increase in $\mathrm{FIO}_{2}$ must be followed by repeat blood gases in 1 hour (or sooner if conscious level deteriorates). * If $\mathrm{pH}$ is $<7.35([\mathrm{H}+]>45 \mathrm{nmol} / \mathrm{L})$ with normal or low PaCO2, investigate and treat for metabolic acidosis and keep $\mathrm{SpO}_{2}$ 94-98\%. ABG, arterial blood gas; COPD, chronic obstructive pulmonary disease; $\mathrm{FiO}_{2}$, fraction of inspired oxygen; $\mathrm{ICU}$, intensive care unit; NIV, non-invasive ventilation; $\mathrm{PaCO}_{2}$, arterial carbon dioxide tension; $\mathrm{PCO}_{2}$, carbon dioxide tension; $\mathrm{PO}_{2}$, oxygen tension; $\mathrm{SpO}_{2}$, arterial oxygen saturation measured by pulse oximetry.

$\checkmark$ Carefully measure respiratory rate and heart rate because tachypnoea and tachycardia are more common than a physical finding of cyanosis in hypoxaemic patients.

$\checkmark$ Appropriate changes should be made to any 'track and trigger' system used to allow for a lower target range in patients at risk of hypercapnic respiratory failure. These patients should score no EWS points for saturation if within their target range and they should score points if the oxygen saturation falls below the target range or if the saturation rises above the target range while breathing oxygen. The 2017 update of the NEWS chart has a special section for oximetry measurements for use with patients who have target range $88-92 \%$ and it is recommended that the 2017 NEWS chart should be used in all hospitals (see recommendation B4). 
Table 2 Serious illnesses requiring moderate levels of supplemental oxygen if the patient is hypoxaemic

\section{Section 8.11}

The initial oxygen therapy is nasal cannulae at 2-6 L/min (preferably) or simple face mask at 5-10 L/min unless stated otherwise.

For patients not at risk of hypercapnic respiratory failure who have saturation below $85 \%$, treatment should be started with a reservoir mask at $15 \mathrm{~L} / \mathrm{min}$ and the recommended initial oxygen saturation target range is $94-98 \%$. If oximetry is not available, give oxygen as above until oximetry or blood gas results are available. Change to reservoir mask if the desired saturation range cannot be maintained with nasal cannulae or simple face mask (and ensure that the patient is assessed by senior medical staff). If these patients have coexisting COPD or other risk factors for hypercapnic respiratory failure, aim at a saturation of $88-92 \%$ pending blood gas results but adjust to $94-98 \%$ if the $\mathrm{PCO}_{2}$ is normal (unless there is a history of previous hypercapnic respiratory failure requiring NIV or IMV) and recheck blood gases after 30-60 min, see table 4.

\begin{tabular}{lll}
\hline & Additional comments & Recommendations \\
\hline Acute hypoxaemia (cause not yet & Reservoir mask at $15 \mathrm{~L} / \mathrm{min}$ if initial $\mathrm{SpO}_{2}$ below $85 \%$, otherwise nasal cannulae or simple face & Recommendations $\mathrm{D} 1-\mathrm{D} 3$
\end{tabular}

diagnosed)

Acute asthma pneumonia lung cancer Deterioration of lung fibrosis or other interstitial lung disease

Pneumothorax

\section{mask}

Patients requiring reservoir mask therapy need urgent clinical assessment by senior staff.

Reservoir mask at $15 \mathrm{~L} / \mathrm{min}$ if initial $\mathrm{SpO}_{2}$ below $85 \%$, otherwise nasal cannulae or simple face mask

Needs aspiration or drainage if the patient is hypoxaemic. Most patients with pneumothorax are not hypoxaemic and do not require oxygen therapy.

Use a reservoir mask at $15 \mathrm{~L} / \mathrm{min}$ if admitted for observation. Aim at $100 \%$ saturation. (Oxygen accelerates clearance of pneumothorax if drainage is not required.)

Pleural effusions Most patients with pleural effusions are not hypoxaemic. If hypoxaemic, treat by draining the effusion as well as giving oxygen therapy.

Pulmonary embolism Most patients with minor pulmonary embolism are not hypoxaemic and do not require oxygen therapy.

Acute heart failure

Severe anaemia

Postoperative breathlessness
Recommendations D1-D3

Recommendations F1-F3 Recommendation F4

Recommendations F5-F6

Recommendation F7

Recommendation F8

Recommendations F9-F10

Recommendations F11-12

Recommendation J1

COPD, chronic obstructive pulmonary disease; CPAP, continuous positive airway pressure; IMV, invasive mechanical ventilation; NIV, non-invasive ventilation; $\mathrm{PCO}_{2}$, arterial or arterialised carbon dioxide tension; $\mathrm{SpO}_{2}$, arterial oxygen saturation measured by pulse oximetry.

Table 3 Conditions for which patients should be monitored closely but oxygen therapy is not required unless the patient is hypoxaemic

\section{Section 8.13}

If hypoxaemic, the initial oxygen therapy is nasal cannulae at 2-6 L/min or simple face mask at 5-10 L/min unless saturation is below $85 \%$ (use reservoir mask) or if at risk from hypercapnia (see below).

The recommended initial target saturation range, unless stated otherwise, is $94-98 \%$.

If oximetry is not available, give oxygen as above until oximetry or blood gas results are available.

If patients have COPD or other risk factors for hypercapnic respiratory failure, aim at a saturation of $88-92 \%$ pending blood gas results but adjust to $94-98 \%$ if the $\mathrm{PCO}_{2}$ is normal (unless there is a history of respiratory failure requiring NIV or IMV) and recheck blood gases after 30-60 min, see table 4 .

\begin{tabular}{|c|c|c|}
\hline & Additional comments & Recommendations \\
\hline $\begin{array}{l}\text { Myocardial infarction and acute coronary } \\
\text { syndromes }\end{array}$ & $\begin{array}{l}\text { Most patients with acute coronary artery syndromes are not hypoxaemic and the benefits/ } \\
\text { harms of oxygen therapy are unknown in such cases. Unnecessary use of high concentration } \\
\text { oxygen may increase infarct size. }\end{array}$ & Recommendation F13 \\
\hline Stroke & $\begin{array}{l}\text { Most patients with stroke are not hypoxaemic. Oxygen therapy may be harmful for } \\
\text { non-hypoxaemic patients with mild-moderate strokes. }\end{array}$ & Recommendation F14 \\
\hline Hyperventilation or dysfunctional breathing & $\begin{array}{l}\text { Exclude organic illness. Patients with pure hyperventilation due to anxiety or panic attacks } \\
\text { are unlikely to require oxygen therapy. } \\
\text { Rebreathing from a paper bag may cause hypoxaemia and is not recommended. }\end{array}$ & See section 8.13 .3 \\
\hline $\begin{array}{l}\text { Most poisonings and drug overdoses (see table } 1 \\
\text { for carbon monoxide poisoning) }\end{array}$ & $\begin{array}{l}\text { Hypoxaemia is more likely with respiratory depressant drugs, give antidote if available, for } \\
\text { example, naloxone for opiate poisoning. } \\
\text { Check blood gases to exclude hypercapnia if a respiratory depressant drug has been taken. } \\
\text { Avoid high blood oxygen levels in cases of acid aspiration as there is theoretical evidence that } \\
\text { oxygen may be harmful in this condition. } \\
\text { Monitor all potentially serious cases of poisoning in a level } 2 \text { or } 3 \text { environment (high } \\
\text { dependency unit or intensive care unit). }\end{array}$ & Recommendation F15 \\
\hline Poisoning with paraquat or bleomycin & $\begin{array}{l}\text { Patients with paraquat poisoning or bleomycin lung injury may be harmed by supplemental } \\
\text { oxygen. } \\
\text { Avoid oxygen unless the patient is hypoxaemic. } \\
\text { Target saturation is } 85-88 \% \text {. }\end{array}$ & Recommendation F16 \\
\hline Metabolic and renal disorders & Most do not need oxygen (tachypnoea may be due to acidosis in these patients) & Recommendation F17 \\
\hline $\begin{array}{l}\text { Acute and subacute neurological and muscular } \\
\text { conditions producing muscle weakness }\end{array}$ & $\begin{array}{l}\text { These patients may require ventilatory support and they need careful monitoring which } \\
\text { includes spirometry. If the patient's oxygen level falls below the target saturation, they need } \\
\text { urgent blood gas measurements and are likely to need ventilatory support. }\end{array}$ & Recommendation G4 \\
\hline Pregnancy and obstetric emergencies & Oxygen therapy may be harmful to the fetus if the mother is not hypoxaemic. & $\begin{array}{l}\text { Recommendations } \\
\mathrm{H} 1-\mathrm{H} 4\end{array}$ \\
\hline
\end{tabular}

COPD, chronic obstructive pulmonary disease; IMV, invasive mechanical ventilation; NIV, non-invasive ventilation; $\mathrm{PCO}_{2}$, arterial or arterialised carbon dioxide tension. 
Table 4 COPD and other conditions requiring controlled or low-dose oxygen therapy

\section{Section 8.12}

Prior to availability of blood gases, use a $24 \%$ Venturi mask at $2-3 \mathrm{~L} / \mathrm{min}$ or $28 \%$ Venturi mask at $4 \mathrm{~L} / \mathrm{min}$ or nasal cannulae at $1-2 \mathrm{~L} / \mathrm{min}$ and aim for an oxygen saturation of $88-92 \%$ for patients with risk factors for hypercapnia but no prior history of respiratory acidosis. Adjust target range to $94-98 \%$ if the $\mathrm{PCO}_{2}$ is normal (unless there is a history of previous NIV or IMV) and recheck blood gases after 30-60 min.

\begin{tabular}{|c|c|c|}
\hline & Additional comments & Recommendations \\
\hline $\begin{array}{l}\text { COPD and other conditions causing fixed } \\
\text { airflow obstruction (eg, bronchiectasis) }\end{array}$ & $\begin{array}{l}\text { May need lower range if acidotic or if known to be very sensitive to oxygen therapy. } \\
\text { Ideally use 'alert cards' to guide therapy based on previous blood gas results. Increase } \\
\text { Venturi mask flow by up to } 50 \% \text { if respiratory rate is above } 30 \text { breaths/min. }\end{array}$ & $\begin{array}{l}\text { Recommendations } \mathrm{G} 1-\mathrm{G} 2 \text { and } \\
\text { section } 8.12 .1\end{array}$ \\
\hline Exacerbation of CF & $\begin{array}{l}\text { Admit to regional CF centre if possible, if not discuss with regional centre or manage } \\
\text { according to protocol agreed with regional CF centre. Ideally use 'alert cards' to guide } \\
\text { therapy. Increase Venturi mask flow by up to } 50 \% \text { if respiratory rate is above } 30 \text { breaths/ } \\
\text { min. }\end{array}$ & Recommendations G1, G3, G6 \\
\hline $\begin{array}{l}\text { Neuromuscular disease, neurological condition } \\
\text { and chest wall deformity }\end{array}$ & $\begin{array}{l}\text { May require ventilatory support. } \\
\text { Risk of hypercapnic respiratory failure }\end{array}$ & Recommendations G1, G4, G6 \\
\hline Morbid obesity & & Recommendations G1,G5, G6 \\
\hline
\end{tabular}

$\checkmark$ The presence of a normal $\mathrm{SpO}_{2}$ does not negate the need for blood gas measurements especially if the patient is on supplemental oxygen therapy. Pulse oximetry will be normal in a patient with normal oxygen tension $\left(\mathrm{PO}_{2}\right)$ but abnormal blood $\mathrm{pH}$ or carbon dioxide tension $\left(\mathrm{PCO}_{2}\right)$ or with a low blood oxygen content due to anaemia. For this reason, blood gases and full blood count tests are required as early as possible in all situations where these measurements may affect patient outcomes.

$\checkmark$ All clinical staff who use oximeters must be trained in their use and made aware of the limitations of oximetry. (Oximetry is a valuable clinical tool but subject to artefact and errors of interpretation).

\section{Arterial and capillary blood gases}

C1: For critically ill patients or those with shock or hypotension (systolic blood pressure $<90 \mathrm{~mm} \mathrm{Hg}$ ), the initial blood gas measurement should be obtained from an arterial sample (see sections 7.1.3 and 8.4 and 8.5). For most patients who require blood gas sampling, either arterial blood gases (ABGs) or arterialised earlobe blood gases may be used to obtain an accurate measure of $\mathrm{pH}$ and $\mathrm{PCO}_{2}$. However, the $\mathrm{PO}_{2}$ is less accurate in earlobe blood gas samples (it underestimates the $\mathrm{PO}_{2}$ by $0.5-$ $1 \mathrm{kPa}$ ) so oximetry should be monitored carefully if earlobe blood gas specimens are used and a repeat arterial specimen should be taken if there is any concern about the accuracy of a capillary sample (grade D).

C2: Local anaesthesia should be used for all ABG specimens except in emergencies (grade A).

C3: Blood gases should be checked in the following situations:

- All critically ill patients (grade D).

- Unexpected or inappropriate fall in $\mathrm{SpO}_{2}$ below 94\% in patients breathing air or oxygen or any patient requiring oxygen to achieve the above target range. (Allowance should be made for transient dips in saturation to $90 \%$ or less in normal participants during sleep) (grade D).

- Deteriorating oxygen saturation (fall of $\geq 3 \%$ ) or increasing breathlessness in a patient with previously stable chronic hypoxaemia (eg, severe COPD) (grade D).

- Most previously stable patients who deteriorate clinically and require increased fraction of inspired oxygen $\left(\mathrm{FiO}_{2}\right)$ to maintain a constant oxygen saturation (grade D).
- Any patient with risk factors for hypercapnic respiratory failure who develops acute breathlessness, deteriorating oxygen saturation, drowsiness or other features of carbon dioxide retention (grade D).

- Patients with breathlessness who are thought to be at risk of metabolic conditions such as diabetic ketoacidosis or metabolic acidosis due to renal failure (grade D).

- Any other evidence from the patient's medical condition that would indicate that blood gas results would be useful in the patient's management (eg, an unexpected change in 'track and trigger' systems such as a sudden rise of several units in the NEWS or an unexpected fall in oxygen saturation of $3 \%$ or more, even if within the target range) (grade D).

Good practice point: patients requiring increased concentration of oxygen

$\checkmark$ The requirement for an increased concentration of oxygen is an indication for urgent clinical reassessment of the patient (and repeat blood gas measurements in most instances, see recommendations W13 and W18 for exceptions).

\section{Initial oxygen therapy; initial choice of equipment for patients who do not have critical illness}

Initial oxygen therapy in critical illness is covered in the next section.

D1: For acutely breathless patients not at risk of hypercapnic respiratory failure who have saturations below $85 \%$, treatment should be started with a reservoir mask at $15 \mathrm{~L} / \mathrm{min}$ in the first instance (see figures 1-2 (charts 1-2) and table 2 and sections 8.9 and 10).* The oxygen concentration can be adjusted downwards (using nasal cannulae at $1-6 \mathrm{~L} / \mathrm{min}$ or a simple face mask at $5-10 \mathrm{~L} / \mathrm{min}$ ) to maintain a target saturation of $94-98 \%$ once the patient has stabilised (grade D).

D2: In other cases of acute hypoxaemia without critical illness or risk factors for hypercapnic respiratory failure, treatment should be started with nasal cannulae (or a simple face mask if cannulae are not tolerated or not effective) with the flow rate adjusted to achieve a saturation of 94-98\% (grade D).

D3: If medium-concentration therapy with nasal cannulae or a simple face mask does not achieve the desired saturation, change to a reservoir mask and seek senior or specialist advice (grade D). 
See patient's drug chart and Chart 1 and tables 1-4 for starting dose and target saturation Choose the most suitable delivery system and flow rate

Titrate oxygen up or down to maintain the target oxygen saturation.

The table below shows available options for stepping dosage up or down.

The chart does NOT imply any equivalence of dose between Venturi masks and nasal cannulae.

Allow at least 5 minutes at each dose before adjusting further upwards or downwards

(except with major and sudden fall in saturation - falls $\geq 3 \%$ also require clinical review)

Once your patient has adequate and stable saturation on minimal oxygen dose, consider discontinuation of oxygen therapy.

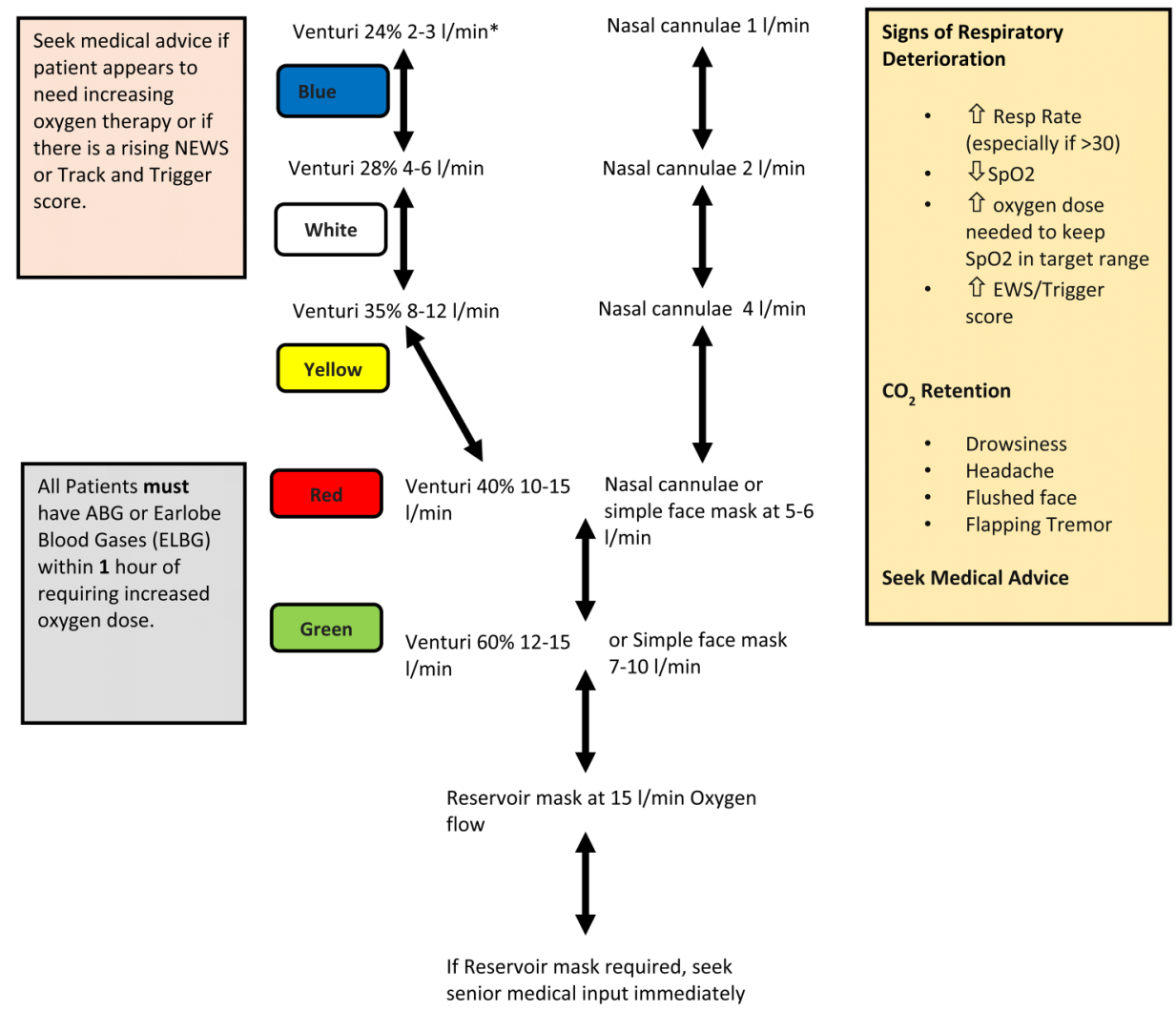

* For Venturi masks, the higher flow rate is required if the respiratory rate is $>30$

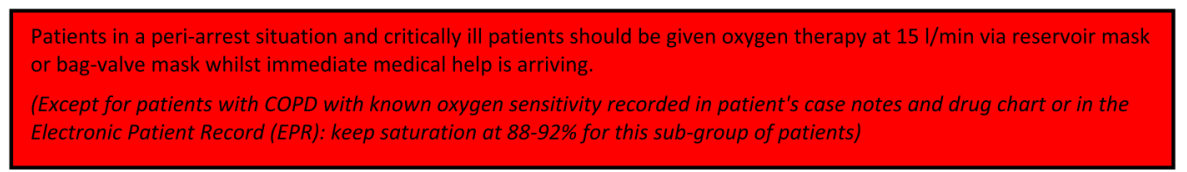

Figure 2 Chart 2 - Flow chart for oxygen administration on general wards in hospitals. * For Venturi masks, the higher flow rate is required if the respiratory rate is $>30$. $A B G$, arterial blood gas; COPD, chronic obstructive pulmonary disease; EPR, electronic patient record; EWS, early warning score; NEWS, National Early Warning Score; $\mathrm{SpO}_{2}$, arterial oxygen saturation measured by pulse oximetry.

\section{Good practice point}

$\checkmark$ High-flow nasal oxygen using specialised equipment should be considered as an alternative to reservoir mask treatment in patients with acute respiratory failure without hypercapnia.

*For initial management of patients at risk of hypercapnic respiratory failure, see recommendations G1 and G2.

\section{E Oxygen therapy in critical illness}

E1: Use the highest feasible inspired oxygen for ventilation during cardiopulmonary resuscitation (CPR; see table 1 and section 8.10). Once spontaneous circulation has returned and arterial blood oxygen saturation can be monitored reliably, aim for a target saturation range of $94-98 \%$ and take an $A B G$ sample to guide ongoing oxygen therapy. If the blood gas shows hypercapnic respiratory failure, reset the target range to 88-92\% or consider mechanical ventilation (grade D).

E2: In critical illness, including major trauma, sepsis, shock and anaphylaxis, initiate treatment with a reservoir mask at $15 \mathrm{~L} / \mathrm{min}$ and aim at a saturation range of $94-98 \%$. This advice also applies to patients with critical illness who have risk factors for hypercapnia pending the results of blood gas measurements and expert assessment. In patients with spontaneous circulation and a reliable oximetry reading it may be possible to maintain a saturation of $94-98 \%$ using lower concentrations of oxygen (grade D) 
E3: In cases of drowning, aim at an oxygen saturation of 9498\% once spontaneous circulation is restored (grade D).

E4: In patients with acute seizures due to epilepsy or other causes, high-concentration oxygen should be administered until a satisfactory oximetry measurement can be obtained and clinicians should then aim for an oxygen saturation of $94-98 \%$ or $88-92 \%$ if the patient is at risk of hypercapnic respiratory failure (grade D).

E5: In cases of major head injury, aim at an oxygen saturation of $94-98 \%$. Initial treatment should involve high-concentration oxygen from a reservoir mask at $15 \mathrm{~L} / \mathrm{min}$ pending availability of satisfactory blood gas measurements or until the airway is secured by intubation (grade D).

E6: In cases of carbon monoxide poisoning, an apparently 'normal' oximetry reading may be produced by carboxyhaemoglobin, so aim at an oxygen saturation of $100 \%$ and use a reservoir mask at $15 \mathrm{~L} / \mathrm{min}$ irrespective of the oximeter reading and arterial oxygen tension $\left(\mathrm{PaO}_{2}\right)$ (grade $\left.\mathrm{D}\right)$.

\section{F Oxygen therapy for specific conditions that frequently require oxygen therapy}

Respiratory conditions with low risk of hypercapnic respiratory failure

F1: In acute asthma, aim at an oxygen saturation of 94-98\% (see tables 2 and 3 and sections 8.11 and 8.13) (grade D).

F2: In cases of pneumonia who are not at risk of hypercapnic respiratory failure, aim at an oxygen saturation of $94-98 \%$ (grade D).

F3: In acute breathlessness due to lung cancer, aim at an oxygen saturation of 94-98\% unless there is coexisting COPD. See also 'Oxygen use in palliative care' section 8.17 (grade D).

F4: In acute deterioration of pulmonary fibrosis or other interstitial lung diseases, aim at an oxygen saturation of 94$98 \%$ or the highest possible if these targets cannot be achieved (grade D).

F5: In most cases of pneumothorax, aim at an oxygen saturation of $94-98 \%$ if the patient is not at risk of hypercapnic respiratory failure (grade D).

F6: In patients with pneumothorax having hospital observation without drainage, the use of high-concentration oxygen $(15 \mathrm{~L} /$ min flow rate via reservoir mask) is recommended unless the patient is at risk of hypercapnic respiratory failure (grade D).

F7: In pleural effusion, aim at an oxygen saturation of 94$98 \%$ (or $88-92 \%$ if the patient is at risk of hypercapnic respiratory failure) (grade D).

F8: In pulmonary embolism, aim at an oxygen saturation of $94-98 \%$ (or $88-92 \%$ if the patient is at risk of hypercapnic respiratory failure) (grade D).

Non-respiratory conditions

F9: In acute heart failure, aim at an oxygen saturation of 94$98 \%$ (or $88-92 \%$ if the patient is at risk of hypercapnic respiratory failure) (grade D).

F10: Continuous positive airway pressure (CPAP) with entrained oxygen or high-flow humidified nasal oxygen to maintain saturation $94-98 \%$ (or $88-92 \%$ if at risk of hypercapnia) should be considered as an adjunctive treatment to improve gas exchange in patients with cardiogenic pulmonary oedema who are not responding to standard treatment (or non-invasive ventilation (NIV) if there is coexistent hypercapnia and acidosis) (grade B).

F11: In anaemia, aim at an oxygen saturation of $94-98 \%$ or $88-92 \%$ if the patient is at risk of hypercapnic respiratory failure (grade D).
Good practice point

$\checkmark$ Correction of anaemia by blood transfusion should be based on national guidelines.

F12: In sickle cell crisis and acute chest syndrome, aim for an oxygen saturation of $94-98 \%$ or aim at the saturation level that is usual for the individual patient (grade D).

Good practice point regarding sickle cell crisis

Arterial or arterialised capillary blood gases should be sampled if there is any doubt about the reliability of oximetry during a sickle cell crisis.

F13: In myocardial infarction and acute coronary syndromes, aim at an oxygen saturation of $94-98 \%$ or $88-92 \%$ if the patient is at risk of hypercapnic respiratory failure (grade D).

F14: High concentrations of oxygen should be avoided in patients with stroke, unless required to maintain normal oxygen saturation. Aim at an oxygen saturation of $94-98 \%$ or $88-92 \%$ if the patient is at risk of hypercapnic respiratory failure (grade D).

Good practice points regarding stroke management

$\checkmark$ Oxygen saturation should be monitored at least every 4 hours throughout the day and night in patients with acute stroke and all episodes of hypoxaemia treated.

$\checkmark$ Patients with hypoxaemia post-stroke require medical review to establish and treat the cause.

$\checkmark$ Oxygen should only be given once the airway has been cleared and at the lowest concentration necessary to achieve an oxygen saturation of $94-98 \%$ or $88-92 \%$ if the patient is at risk of hypercapnic respiratory failure.

$\checkmark$ Oxygen should be given via nasal cannulae, unless there are clear indications for a different oxygen delivery system.

$\checkmark$ Patients with stroke and cardiorespiratory comorbidities should be positioned as upright as possible, in a chair if possible (see recommendation A5).

$\checkmark$ Patients with a reduced level of consciousness after stroke should be nursed in the recovery position with the paralysed side lowest.

\section{Suspected hyperventilation}

Good practice points regarding patients with suspected byperventilation

$\checkmark$ Organic illness must be excluded before making a diagnosis of hyperventilation.

$\checkmark$ Patients with a definite diagnosis of hyperventilation should have their oxygen saturation monitored. Those with normal or high $\mathrm{SpO}_{2}$ do not require oxygen therapy.

$\checkmark$ Rebreathing from a paper bag can be dangerous and is NOT advised as a treatment for hyperventilation.

F15: In most poisonings, aim at an oxygen saturation of 94 $98 \%$ unless the patient is at risk of hypercapnic respiratory failure (grade D).

F16: In poisoning by paraquat and poisoning by bleomycin, give oxygen only if the saturation falls below $85 \%$ and reduce or stop oxygen therapy if the saturation rises above $88 \%$ (grade D).

F17: In most metabolic and renal disorders, aim at an oxygen saturation of $94-98 \%$ unless the patient is at risk of hypercapnic respiratory failure (grade D).

F18: For patients with cluster headaches, oxygen should be administered using a flow of at least $12 \mathrm{~L} / \mathrm{min}$ from a reservoir mask and home oxygen should be provided (grade D).

\section{G Patients at risk of hypercapnic respiratory failure (See} table 4 and section 8.12 )

G1 (also A3): For most patients with known COPD or other known risk factors for hypercapnic respiratory failure (eg, morbid obesity, CF, chest wall deformities or neuromuscular 
disorders or fixed airflow obstruction associated with bronchiectasis), a target saturation range of $88-92 \%$ is suggested pending the availability of blood gas results (grade A for COPD, grade D for other conditions).

G2: Some patients with COPD and other conditions are vulnerable to repeated episodes of hypercapnic respiratory failure. In these cases it is recommended that treatment should be based on the results of previous blood gas estimations during acute exacerbations. For patients with prior hypercapnic failure (requiring NIV or intermittent positive pressure ventilation) who do not have an alert card, it is recommended that lowconcentration oxygen treatment should be started using a $24 \%$ Venturi mask at $2-3 \mathrm{~L} / \mathrm{min}$ (or a $28 \%$ Venturi mask at $4 \mathrm{~L} / \mathrm{min}$ or nasal cannulae at $1-2 \mathrm{~L} / \mathrm{min}$ if a $24 \%$ mask is not available) with an initial target saturation of $88-92 \%$ pending urgent blood gas results. These patients should be treated as a high priority by emergency services and the oxygen concentration should be reduced if the saturation exceeds $92 \%$ but increased if it falls below $88 \%$ (grade D).

Good practice points for COPD and other conditions that may cause hypercapnic respiratory failure

Diagnosis of COPD or suspected exacerbation of COPD

$\checkmark$ If the diagnosis is unknown, patients over 50 years of age who are long-term smokers with a history of chronic breathlessness on minor exertion such as walking on level ground and no other known cause of breathlessness should be treated as having suspected COPD for the purposes of this guideline.

$\checkmark$ Spirometry should be measured at least once during hospital admissions for suspected COPD (as per National Institute of Health and Care Excellence (NICE) COPD guideline ${ }^{1}$ ). Measurement of spirometry may confirm (or exclude) a diagnosis of airflow obstruction and the forced expiratory volume in $1 \mathrm{~s}\left(\mathrm{FEV}_{1}\right)$ level is a useful indicator of disease severity in COPD.

Immediate management of patients with known or suspected COPD

$\checkmark$ If the saturation remains below $88 \%$ in prehospital care despite a $28 \%$ Venturi mask, change to nasal cannulae at 2$6 \mathrm{~L} / \mathrm{min}$ or a simple face mask at $5 \mathrm{~L} / \mathrm{min}$ with target saturation of $88-92 \%$ and alert the accident and emergency (A\&E) department that the patient is to be treated as a high priority.

$\checkmark$ Patients with a respiratory rate $>30$ breaths/min should have the flow rate from Venturi masks set above the minimum flow rate specified for the Venturi mask packaging to compensate for the patient's increased inspiratory flow (see figure 11B). Increasing the oxygen flow rate into a Venturi mask does not increase the concentration of oxygen which is delivered.

$\checkmark$ Patients with a significant likelihood of severe COPD or other illness that may cause hypercapnic respiratory failure should be triaged as very urgent on arrival in hospital emergency departments and blood gases should be measured on arrival in hospital.

$\checkmark$ Prior to availability of blood gas measurements, use a $24 \%$ Venturi mask at $2-3 \mathrm{~L} / \mathrm{min}$ or nasal cannulae at $1-2 \mathrm{~L} / \mathrm{min}$ or $28 \%$ Venturi mask at $4 \mathrm{~L} / \mathrm{min}$ and aim for an oxygen saturation of $88-92 \%$.

Initial hospital management of patients with exacerbation of COPD

$\checkmark$ Patients with exacerbations of COPD need careful monitoring for hypercapnic respiratory failure with respiratory acidosis which may develop in the course of a hospital admission even if the initial blood gases were satisfactory. $\checkmark$ Avoid excessive oxygen use in patients with COPD. The risk of respiratory acidosis in patients with hypercapnic respiratory failure is increased if the $\mathrm{PaO}_{2}$ is above $10.0 \mathrm{kPa}$ due to previous excessive oxygen use.

$\checkmark$ If following blood gas measurements the $\mathrm{pH}$ and $\mathrm{PCO}_{2}$ are normal, aim for an oxygen saturation of 94-98\% unless there is a history of previous hypercapnic respiratory failure requiring NIV or intermittent positive pressure ventilation or if the patient's usual oxygen saturation when clinically stable is below 94\% (these patients should have a target range of $88-92 \%)$. Blood gases should be repeated at 30-60 min to check for rising $\mathrm{PCO}_{2}$ or falling $\mathrm{pH}$.

$\checkmark$ Recheck blood gases after 30-60 min (or if there is evidence of clinical deterioration) for all patients with COPD or other risk factors for hypercapnic respiratory failure even if the initial $\mathrm{PCO}_{2}$ measurement was normal.

$\checkmark$ If the $\mathrm{PCO}_{2}$ is raised but $\mathrm{pH}$ is $\left.\geq 7.35\left(\mathrm{H}^{+}\right] \leq 45 \mathrm{nmol} / \mathrm{L}\right)$ and/ or a high bicarbonate level $(>28 \mathrm{mmol} / \mathrm{L})$, the patient has probably got long-standing hypercapnia; maintain target range of $88-92 \%$ for these patients. Blood gases should be repeated at $30-60 \mathrm{~min}$ to check for rising $\mathrm{PCO}_{2}$ or falling $\mathrm{pH}$.

$\checkmark$ If the patient is hypercapnic $\left(\mathrm{PCO}_{2}>6 \mathrm{kPa}\right.$ or $\left.45 \mathrm{~mm} \mathrm{Hg}\right)$ and acidotic $\left(\mathrm{pH}<7.35\right.$ or $\left.\left[\mathrm{H}^{+}\right]>45 \mathrm{nmol} / \mathrm{L}\right)$, start NIV with targeted oxygen therapy if respiratory acidosis persists for more than $30 \mathrm{~min}$ after initiation of standard medical management.

$\checkmark$ For patients using Venturi masks, consider changing from Venturi mask to nasal cannulae once the patient has stabilised.

$\checkmark$ For patients who use long-term home oxygen (LTOT) for severe COPD, a senior clinician should consider setting a patient-specific target range if the standard range of $88-92 \%$ would require inappropriate adjustment of the patient's usual oxygen therapy while the patient is in hospital.

\section{Good practice points}

Management of hypercapnia or respiratory acidosis due to excessive oxygen therapy (avoidance of life-threatening rebound hypoxaemia)

$\mathcal{J}$ If a patient is suspected to have hypercapnic respiratory failure due to excessive oxygen therapy, the oxygen therapy must be stepped down to the lowest level required to maintain a saturation range of $88-92 \%$. This may be achieved using $28 \%$ or $24 \%$ oxygen from a Venturi mask or $1-2 \mathrm{~L} /$ min via nasal cannulae depending on oxygen saturation and subsequent blood gas measurements.

$\checkmark$ Sudden cessation of supplementary oxygen therapy can cause life-threatening rebound hypoxaemia with a rapid fall in oxygen saturations below the starting oxygen saturation prior to the start of supplementary oxygen therapy.

G3: Initial oxygen treatment of CF exacerbations should be similar to the initial oxygen treatment of COPD exacerbations with target saturation $88-92 \%$ (see sections 8.12.1-8.12.2; grade D).

G4: In the initial management of musculoskeletal and neurological disorders with acute respiratory failure or acute-on-chronic respiratory failure, aim at an oxygen saturation of $88-92 \%$ and measure blood gases to determine if NIV will be required (grade D).

Good practice point regarding patients with neurological disorders

$\checkmark$ Patients with respiratory failure due to neurological disorders or muscle disease are at high risk of dying and require urgent 
assessment to determine if they are likely to require noninvasive or invasive ventilator support rather than oxygen therapy. Monitor these patients with blood gases and regular spirometry (forced vital capacity). Patient's wishes regarding this form of treatment should be established as early as possible in the course of the illness, ideally before an acute episode has developed.

G5: Morbidly obese patients (body mass index (BMI) $>40 \mathrm{~kg} /$ $\mathrm{m}^{2}$ ), even without evidence of coexistent obstructive sleep apnoea (OSA) are at risk of hypoventilation and should be given titrated oxygen to maintain a target saturation of $88-92 \%$ (grade D).

G6: NIV should be considered for hypercapnic patients with COPD, CF, neuromuscular disorders or morbid obesity who are at risk of hypercapnic respiratory failure if the $\mathrm{pH}$ is $<7.35$ or $\left[\mathrm{H}^{+}\right]>45 \mathrm{nmol} / \mathrm{L}$ (grade D). See BTS/ICS Guideline for the ventilatory management of acute hypercapnic respiratory failure (ref 299).

\section{H Oxygen use during pregnancy (See section 8.14)}

H1: Women who suffer from major trauma, sepsis or acute illness during pregnancy should receive the same oxygen therapy as any other seriously ill patients, with a target oxygen saturation of $94-98 \%$. The same target range should be applied to women with hypoxaemia due to acute complications of pregnancy (eg, collapse related to amniotic fluid embolus, eclampsia or antepartum or postpartum haemorrhage) (grade D).

H2: Women with underlying hypoxaemic conditions (eg, heart failure) should be given supplemental oxygen during labour to achieve an oxygen saturation of 94-98\% unless they are at risk of hypercapnic respiratory failure (target range 8892\%) (grade D).

H3: Pregnant women who are fully conscious with no cardiovascular compromise may be managed in the sitting position or if lying down should use the full left lateral position (grade D).

H4: Pregnant women above 20 weeks gestation (uterine fundus at or above the level of the umbilicus) who are at risk of developing associated cardiovascular compromise (eg, trauma, vaginal bleeding, etc) should be positioned to avoid aortocaval compression by using left lateral tilt, manual uterine displacement or by placing them in a full left lateral position (grade D).

H5: Women who are more than 20 weeks pregnant with evidence of hypoxaemia associated with reduced consciousness or those requiring respiratory or cardiovascular support or CPR should be managed with left lateral tilt or manual uterine displacement (ideally to the left) to improve cardiac output and oxygen delivery (grade D).

H6: The use of oxygen supplementation during intrauterine fetal resuscitation during labour was widespread in the past but there is no evidence of benefit. There is weak evidence of harm to the fetus if supplemental oxygen is given for long periods during uncomplicated labour. Overall, the use of oxygen during labour is only required when there is evidence of maternal hypoxaemia (oxygen saturation <94\%) (grade D).

\section{J Oxygen use in perioperative care and during procedures requiring conscious sedation (see sections $8.15-8.16$ and 10.11)}

J1: Hyperoxaemia is not recommended routinely in the perioperative and postoperative period to reduce the incidence of postoperative nausea and vomiting (grade D).

J2: All procedures involving conscious sedation warrant routine continuous monitoring of oxygen saturation via pulse oximetry prior to and during the procedure, and in the recovery period, particularly fibre optic bronchoscopy and upper gastrointestinal (GI) endoscopy where a reduction in arterial oxygen saturation $\left(\mathrm{SaO}_{2}\right)$ is common, particularly with concurrent use of sedation (grade C).

J3: Significant arterial oxygen desaturation $\left(\mathrm{SpO}_{2}<90 \%\right.$ or fall of $4 \%$ or more that is prolonged ( $>1$ min during endoscopy procedures)) should be corrected by supplemental oxygen with the aim of achieving target oxygen saturations of $94-98 \%$, or $88-92 \%$ in those at risk of hypercapnic respiratory failure (grade D).

J4: Complicated upper GI endoscopy or procedures in patients with cardiorespiratory comorbidity are especially likely to lead to hypoxaemia and may also lead to hypercapnia, especially if the patient is heavily sedated. It is recommended that blood gases should be measured if such patients should require prolonged oxygen administration. The routine administration of oxygen is not recommended as it may delay the recognition of respiratory failure (grade $\mathrm{D}$ ).

J5: Constant clinical assessment of the patient is crucial at all stages of conscious sedation procedures and monitoring of capnography or transcutaneous carbon dioxide levels may be a useful adjunct to identify early respiratory depression (grade D).

J6: During the recovery period after procedures requiring conscious sedation, supplemental oxygen should be titrated to achieve target saturations of $94-98 \%$ in most patients and $88-$ $92 \%$ in those at risk of hypercapnic respiratory failure (see 10.5.1) (grade D).

Good practice points related to oxygen use in perioperative care

$\checkmark$ A target saturation of $94-98 \%$ is recommended for most surgical patients except those at risk of hypercapnic respiratory failure when a range of $88-92 \%$ should be achieved.

$\checkmark$ Pulse oximetry monitoring is recommended for postoperative patients despite the lack of evidence from randomised studies.

$\checkmark$ Patients using patient controlled analgesia (PCA) should have two-hourly oximetry observations because of the risk of hypoxaemia. Oxygen should be administered to keep patients within the appropriate target saturation range.

$\checkmark$ A target saturation of $94-98 \%$ is advised in most patients having PCA except those at risk of hypercapnic respiratory failure when a range of $88-92 \%$ should be achieved.

$\checkmark$ There is conflicting evidence concerning the balance of potential benefits and risks of perioperative hyperoxaemia to reduce the risk of surgical site infection in elective surgery and there is no evidence for this practice in patients having emergency surgical procedures. More trials are required for specific procedures and more information is required concerning long-term mortality risks to patients with cancer. In the meantime, oxygen should not be used for this indication outside of clinical trials.

\section{K Oxygen use in palliative care (see section 8.17 )}

K1: Oxygen use in palliative care patients should be restricted to patients with $\mathrm{SpO}_{2}$ consistently $<90 \%$ or patients who report significant relief of breathlessness from oxygen. In nonhypoxaemic patients, opioids and non-pharmacological measures should be tried before oxygen (grade B).

K2: In general, there is no role for the monitoring of oxygen saturation or $\mathrm{PaO}_{2}$ in comfort-focused care in the last few days of life. If the patient appears comfortable, oxygen levels are irrelevant and should not influence care (grade D). 
Good practice points related to oxygen use in palliative care

Oxygen therapy for the symptomatic relief of breathlessness in palliative care patients is more complex than the simple correction of hypoxaemia. Consider the following issues:

$\checkmark$ Consider early involvement of palliative care specialists and physiotherapists;

$\checkmark$ As breathlessness is a multifactorial sensation-a comprehensive assessment of contributing factors (such as anxiety) should be carried out.

$\checkmark$ Low-dose opioids should be considered because they are effective for the relief of breathlessness in palliative care patients.

$\checkmark$ A trial of a hand held fan to help relieve breathlessness is recommended prior to trial of oxygen.

$\checkmark$ Oxygen use has to be tailored to the individual and a formal assessment made of its efficacy for reducing breathlessness and improving quality of life for that person.

$\checkmark$ Oxygen therapy should not be continued in the absence of patient benefit or where its disadvantages (eg, discomfort of masks or nasal cannulae, drying of mucous membranes) outweigh any likely symptomatic benefit.

\section{Mixtures of oxygen with other gases (Heliox and Entonox)}

Use of helium-oxygen mixtures (Heliox) see section 8.18

L1: There is insufficient evidence to support the use of Heliox either as an inhaled gas or as the driving gas for nebuliser therapy in adult patients with acute exacerbations of asthma or acute exacerbations of COPD (AECOPD) except as part of randomised clinical trials or in exceptional circumstances (grade D).

L2: A therapeutic trial of Heliox is reasonable in patients with mechanical upper airway obstruction or postoperative stridor (grade D).

L3: Heliox use for patients with asthma or COPD should be considered only in clinical trials or in specialist hands for severe exacerbations that are not responding to standard treatment (and in patients with COPD where there are contraindications to intubation) (grade D)

\section{Use of nitrous oxide/oxygen mixtures (Entonox) for analgesia (see section 9.11)}

M1: The use of Entonox gas mixture for analgesia should be avoided if possible in patients at risk of hypercapnic respiratory failure (grade D).

\section{N CPAP and humidified high-flow nasal oxygen}

Use of CPAP in the perioperative period and for pulmonary oedema (see section 8.19)

N1: Patients with diagnosed sleep-disordered breathing established on CPAP undergoing surgery should bring their machines with them and use them in the preoperative and postoperative period. If adequate saturations are not achieved despite CPAP therapy then assess for worsening ventilation with blood gases and oxygen should be entrained to achieve a saturation of 88-92\% (grade D).

N2: CPAP with entrained oxygen to maintain saturation 94-98\% should be considered as an adjunctive treatment to improve gas exchange in patients with cardiogenic pulmonary oedema who are not responding to standard treatment in hospital care or in prehospital care (grade B).

Good practice point, high-flow humidified nasal oxygen via nasal cannulae

$\checkmark$ High-flow humidified nasal oxygen should be considered as a potentially superior alternative to reservoir mask treatment in patients with acute respiratory failure without hypercapnia.

The prefix $\mathrm{O}$ is not in use for recommendations.

\section{P Patients with tracheostomy or laryngectomy (see section 10.3)}

P1: When oxygen is required by patients with prior tracheostomy or laryngectomy, a tracheostomy mask (varying the flow as necessary) should achieve the desired oxygen saturation (tables 1-4). An alternative delivery device, usually a T-piece device fitted directly to the tracheostomy tube, may be necessary if the patient deteriorates (grade D).

\section{Q Humidification of oxygen (see section 10.2)}

Q1: Humidification is not required for the delivery of low-flow oxygen (mask or nasal cannulae) or for the short-term use of high-flow oxygen. It is not therefore required in prehospital care. Pending the results of clinical trials, it is reasonable to use humidified oxygen for patients who require high-flow oxygen systems for more than 24 hours or who report upper airway discomfort due to dryness (grade D).

Q2: In the emergency situation, humidified oxygen use can be confined to patients with tracheostomy or an artificial airway although these patients can be managed without humidification for short periods of time (eg, ambulance journeys) (grade D).

Q3: Humidification may also be of benefit to patients with viscous secretions causing difficulty with expectoration. This benefit can be achieved using nebulised normal saline (grade D).

Q4: Bubble bottles which allow a stream of oxygen to bubble through a container of water should not be used because there is no evidence of a clinically significant benefit but there is a risk of infection (grade D).

Good practice points related to humidified oxygen therapy

$\checkmark$ Consider use of a large volume oxygen humidifier device for patients requiring high-flow rates or longer term oxygen, especially if sputum retention is a clinical problem.

$\checkmark$ In the absence of an artificial airway the decision to humidify supplemental oxygen needs to be made on an individual basis but this practice is not evidence-based.

\section{R Driving gas for nebulised treatments (see section 10.4)}

R1: For patients with asthma, nebulisers should be driven by piped oxygen or from an oxygen cylinder fitted with a highflow regulator capable of delivering a flow rate of $>6 \mathrm{~L} / \mathrm{min}$. The patient should be changed back to his/her usual oxygen mask or cannulae when nebuliser therapy is complete. If the cylinder does not produce this flow rate, an air-driven nebuliser (with electrical compressor) should be used with supplemental oxygen by nasal cannulae at $2-6 \mathrm{~L} / \mathrm{min}$ to maintain an appropriate oxygen saturation level (grade D).

$R 2$ : When nebulised bronchodilators are given to patients with hypercapnic acidosis, they should be given using an ultrasonic nebuliser or else a jet nebuliser driven by compressed air and, if necessary, supplementary oxygen should be given concurrently by nasal cannulae to maintain an oxygen saturation of 88-92\%. The same precautions should be applied to patients who are at risk of hypercapnic respiratory failure prior to the availability of blood gas results and the oxygen saturation should be monitored continuously during treatment. Once the nebulised treatment is completed for patients at risk of hypercapnic respiratory failure, their previous targeted oxygen therapy should be reinstituted (grade D). 


\section{Good practice points}

Do not allow hypoxaemia to occur while administering nebulised treatments:

$\checkmark$ For hypoxaemic patients, oxygen therapy should continue during nebulised treatments.

Driving gas for nebulised treatment in ambulances

$\checkmark$ During treatment by ambulance staff oxygen-driven nebulisers should be used for patients with asthma and may be used for patients with COPD in the absence of an air-driven compressor system. If oxygen is used for patients with known COPD, its use should be limited to $6 \mathrm{~min}$. This will deliver most of the nebulised drug dose but limit the risk of hypercapnic respiratory failure (section 10.4). Ambulance services are encouraged to explore the feasibility of introducing battery-powered, air-driven nebulisers or portable ultrasonic nebulisers.

\section{S Prescribing oxygen therapy (see section 11)}

S1: Every healthcare facility should have a standard oxygen prescription document or, preferably, a designated oxygen section on all drug-prescribing cards or guided prescription of oxygen in electronic prescribing systems (grade D).

S2: A prescription for oxygen should always be provided, except in sudden illness when it must be started immediately and documented retrospectively (grade D).

S3: Doctors and other prescribers should prescribe oxygen using a target saturation range (sections 8, 9 and 11) and sign the drug chart or electronic prescribing order (grade D).

S4: An oxygen target saturation range should be prescribed for all patients who are admitted to hospital. This will ensure that every patient will receive appropriate oxygen therapy if it should be required. It will also ensure that all clinicians are aware of the appropriate oxygen target range for every patient under their care (grade D).

Good practice points related to prescribing and administering oxygen therapy to patients

$\checkmark$ Oxygen should be prescribed on the drug chart or electronic prescribing system using a target saturation range.

$\checkmark$ Oxygen should be prescribed to a target saturation range rather than prescribing a fixed concentration of oxygen or $\mathrm{FiO}_{2}$ (see recommendations A1, A2, A4 and A5)

$\checkmark$ In most emergency situations, oxygen is given to patients immediately without a formal prescription. The lack of a prescription should never preclude oxygen being given when needed in an emergency situation. However, a subsequent written record must be made of what oxygen therapy has been given to every patient in a similar manner to the recording of all other emergency treatment.

$\checkmark$ If a patient has an oxygen alert card, initial oxygen therapy should be based on the guidance on the card until the results of blood gases are available.

T Monitoring and adjusting oxygen therapy (see sections 9-11) T1: Pulse oximetry must be available in all locations where emergency oxygen is being used by healthcare professionals (see also the limitations of using pulse oximetry section 7.1.2) (grade D).

T2: All documents which record oximetry measurements or blood gas results should state whether the patient is breathing air or a specified oxygen delivery device and flow rate using the abbreviations shown in table 5 (grade D).

T3: In all situations where repeated blood gas measurements are required, they should be measured as soon as possible, usually within $30 \mathrm{~min}$ of any treatment change, to determine if the proposed target saturations are appropriate. Consider the use of an indwelling arterial catheter if multiple samples are likely to be required (grade D).

T4: Adjustments should only be made by registered staff who have been trained to administer oxygen. If the oxygen saturation falls below the prespecified range, the concentration of oxygen should be increased; if the saturation rises above this range, the oxygen concentration should be reduced. If the monitoring of oxygen saturation is performed by unregistered staff (eg, healthcare assistants), there must be a clear protocol in place which requires that they should inform staff who are trained to administer oxygen if the oxygen saturation is above or below the target saturation (grade D).

Good practice points related to administration of oxygen therapy

$\checkmark$ For hypoxaemic patients, oxygen therapy should continue during other treatments such as nebulised therapy. Clinicians should assess the clinical status of the patient prior to prescribing oxygen and the patient's condition should be reassessed frequently during oxygen use (see recommendations B1-B3).

$\checkmark$ The administering healthcare professional should note the oxygen saturation before starting oxygen therapy whenever possible but never discontinue or delay oxygen therapy for seriously ill patients (see recommendation B2).

$\checkmark$ The healthcare professional should start oxygen therapy using an appropriate delivery system and flow rate as specified in sections $8-10$ of this guideline. The target oxygen saturation should be documented on the respiratory section of the observation chart.

Table 5 Abbreviations for oxygen devices for use on bedside charts

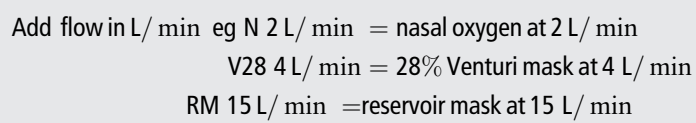

A, air

$\mathrm{N}$, nasal cannulae

V24, Venturi mask $24 \%$

V40, Venturi mask $40 \%$

$\mathrm{H} 28$, humidified $\mathrm{O}_{2} 28 \%$

$\mathrm{RM}$, reservoir mask

CPAP, continuous positive airway pressure
HFN, high-flow humidified oxygen via nasal cannulae

V28, Venturi mask $28 \%$

V60, Venturi mask $60 \%$

$\mathrm{H} 40$, humidified $\mathrm{O}_{2} 40 \%$

SM, simple face mask

NIV, non-invasive ventilation
V35, Venturi mask $35 \%$

$\mathrm{H} 60$, humidified $\mathrm{O}_{2} 60 \%$

$\mathrm{TM}$, tracheostomy mask 
$\checkmark$ Whenever possible, patients should be given an oxygen information sheet (example in web appendix 6 of this guideline on the BTS website).

$\checkmark$ Staff should check the oxygen supply and connections on a regular basis because there have been serious incidents due to disconnection or misconnection of oxygen supplies.

$\checkmark$ Staff must ensure that adequate oxygen is provided during transfers and while patients are in diagnostic departments. Additionally, oxygen saturation should be monitored continuously for seriously ill patients who require escorted transfers. This is because there have been serious incidents involving accidental discontinuation of oxygen or cylinders running out during interward transfers or transfers to other departments such as for x-rays.

\section{U Weaning and discontinuation of oxygen therapy}

U1: Lower the oxygen concentration if the patient is clinically stable and the oxygen saturation is above the target range or if it has been in the upper zone of the target range for some time (usually 4-8 hours) (grade D).

$U 2$ : If the target saturation is maintained, the new delivery system and flow should be continued. Repeat blood gas measurements are not required. If the patient is stable the process can be repeated and the patient can eventually be weaned off oxygen (see section 12) (grade D).

U3: Most stable convalescent patients will eventually be stepped down to $2 \mathrm{~L} / \mathrm{min}$ via nasal cannulae prior to cessation of oxygen therapy. Patients at risk of hypercapnic respiratory failure may be stepped down to $1 \mathrm{~L} / \mathrm{min}$ (or occasionally $0.5 \mathrm{~L}$ / min) via nasal cannulae or a $24 \%$ Venturi mask at $2 \mathrm{~L} / \mathrm{min}$ as the lowest oxygen concentration prior to cessation of oxygen therapy (grade D).

U4: Oxygen therapy should be stopped once a patient is clinically stable on low-concentration oxygen and the oxygen saturation is within the desired range on two consecutive observations (but the prescription for a target saturation range should remain active in case of future deterioration). It may be appropriate to alter the target range following senior review in patients with chronic cardiopulmonary disease who either have saturations $<94 \%$ when stable or in whom it is deemed sensible to discharge from hospital with saturations $<94 \%$ pending an outpatient oxygen assessment. Oxygen should also be stopped if the patient has come to the end of a written protocol of timed oxygen (eg, postoperatively) (grade D).

U5: Oxygen saturation on air should be monitored for $5 \mathrm{~min}$ after stopping oxygen therapy. If it remains in the desired range it should be rechecked at 1 hour (grade D).

U6: If the oxygen saturation and physiological 'track and trigger' score (eg, NEWS) is satisfactory at 1 hour, the patient has safely discontinued oxygen therapy. However, saturation and physiology should continue to be monitored on a regular basis according to the patient's underlying clinical condition (grade D).

U7: If the saturation falls below the patient's target range on stopping oxygen therapy, restart the lowest concentration that maintained the patient in the target range and monitor for $5 \mathrm{~min}$. If this restores the saturation into the target range, continue oxygen therapy at this level and attempt discontinuation of oxygen therapy again at a later date provided the patient remains clinically stable (grade $\mathrm{D})$.

U8: If a patient requires oxygen therapy to be restarted at a higher concentration than before to maintain the same target saturation range, the patient should have a clinical review to establish the cause for this deterioration (grade D).
U9: Some patients may have episodic hypoxaemia (eg, after minor exertion or due to mucus plugging) after they have safely discontinued oxygen therapy. An ongoing prescription for a target saturation range will allow these patients to receive oxygen as the need arises but transient asymptomatic desaturation does not require correction (grade D).

\section{Practical aspects of oxygen use in prehospital and hospital care and use of oxygen alert cards (see sections 9-11)}

V1: Emergency oxygen should be available in primary care medical centres, preferably using oxygen cylinders with integral high-flow regulators. Alternatively, oxygen cylinders fitted with high-flow regulators (delivering up to $15 \mathrm{~L} / \mathrm{min}$ ) must be used to allow use with reservoir masks (grade D).

V2: Healthcare organisations should take measures to eliminate the risk of oxygen tubing being connected to the incorrect wall oxygen outlet or to outlets that deliver compressed air or other gases instead of oxygen. Air flow meters should be removed from the wall sockets or covered with a designated air outlet cover when not in use. Special care should be taken if twin oxygen outlets are in use (grade D).

Good practice points related to practical aspects of oxygen therapy

Assessment and immediate oxygen therapy

$\checkmark$ Chronically hypoxaemic patients with a clinical exacerbation associated with a $3 \%$ or greater fall in oxygen saturation on their usual oxygen therapy should usually be assessed in hospital with blood gas estimations. $\mathrm{PaO}_{2}$ of $<7 \mathrm{kPa}$ equates to $\mathrm{SpO}_{2}$ below $\sim 85 \%$.

$\checkmark$ The initial oxygen therapy to be used in the various clinical situations is given in tables $1-4$.

$\checkmark$ If there is a clear history of asthma or heart failure or other treatable illness, appropriate treatment should be instituted in accordance with guidelines or standard management plans for each disease.

$\checkmark$ The oxygen saturation should be monitored continuously until the patient is stable or arrives at hospital for a full assessment. The oxygen concentration should be adjusted upwards or downwards to maintain the target saturation range.

$\checkmark$ In most emergency situations oxygen is given to patients immediately without a formal prescription or drug order. The lack of a prescription should never preclude oxygen being given when needed in an emergency situation. However, a subsequent written record must be made of what oxygen therapy has been given to every patient (in a similar manner to the recording of all other emergency treatment).

$\checkmark$ General practitioners (GPs) or first responders visiting a patient's home should carry a portable pulse oximeter to assess hypoxaemia and guide use of oxygen if available and should call emergency services if hypoxaemia or other serious illness is suspected.

$\checkmark$ Those attending patients as an emergency in rural or remote areas should consider carrying a portable oxygen cylinder as part of their emergency equipment.

Oxygen alert cards for patients with hypercapnic respiratory failure

$\checkmark$ Patients with COPD (and other at-risk conditions) who have had an episode of hypercapnic respiratory failure should be issued with an oxygen alert card and with a $24 \%$ or $28 \%$ Venturi mask. They should be instructed to show the card to the ambulance crew and emergency department staff in the event of an exacerbation. 
$\checkmark$ Oxygen alert cards with agreed content can be obtained via the BTS website.

$\checkmark$ The content of the alert card should be specified by the physician in charge of the patient's care, based on previous blood gas results.

$\checkmark$ The primary care team and ambulance service should also be informed by the hospital COPD team that the patient has had an episode of hypercapnic respiratory failure and carries an oxygen alert card. The home address and ideal oxygen concentration or target saturation ranges of these patients can be flagged in the ambulance control systems and information disseminated to ambulance crews when required.

$\checkmark$ When possible, out-of-hours services providing emergency primary care services should be informed by the hospital COPD team or by the primary care team that the patient has had an episode of hypercapnic respiratory failure and carries an oxygen alert card. Use of oxygen in these patients will be guided by the instructions on the alert card or by a patient-specific protocol which can be shared by hospital teams, the ambulance service and the primary care team.

\section{W Practical aspects of oxygen dispensing, documentation and monitoring}

W1: Registered nurses and others who dispense drugs in hospitals should sign the drug chart or electronic prescribing record at every drug round and check that the patient is receiving oxygen therapy. This is to check that the patient is within the target saturation and also to check whether weaning and discontinuation should be instituted (grade D).

W2: Most patients are prescribed an oxygen target range. If patients are on air at the time of the drug round, registered nurses should sign the drug chart using a code such as 'A' for air and the observation chart should also be filled in using the code A for air (see table 5 and figure 19) (grade D).

W3: All patients should have their oxygen saturation observed for at least $5 \mathrm{~min}$ after starting oxygen therapy or for patients who require an increased concentration of oxygen and after oxygen therapy has been decreased or stopped (grade D).

W4: If the oxygen saturation is above the target saturation range and the patient is stable, the delivery system or oxygen flow rate should be modified to return the saturation to within the target range (grade D).

W5: Patients who have a target saturation of $88-92 \%$ should have their blood gases measured within $30-60 \mathrm{~min}$. This is to ensure that the carbon dioxide level is not rising. This recommendation also applies to those who are at risk of developing hypercapnic respiratory failure but who have a normal $\mathrm{PCO}_{2}$ on the initial blood gas measurement (grade D).

W6: Stable patients whose oxygen saturation is within their target saturation range of $94-98 \%$ do not need repeat blood gas measurements within 30-60 min if there is no risk of hypercapnic respiratory failure and acidosis and may not need any further blood gas measurements unless there should be further deterioration including symptoms or signs of possible hypercapnia (grade D).

W7: Stable patients on oxygen treatment should have $\mathrm{SpO}_{2}$ and physiological variables (eg, NEWS) measured four times a day (grade D).
W8: In those who have signs of critical illness (eg, NEWS 7 or above), oxygen saturation should be monitored continuously and the patient may require level 2 or 3 care on a HDU or critical care unit (grade D).

W9: If the patient is clinically stable and the oxygen saturation is within the target range, treatment should be continued (or eventually lowered) depending on the clinical situation (grade D).

W10: Oxygen therapy should be increased if the saturation is below the desired range and decreased if the saturation is above the desired range (and eventually discontinued as the patient recovers) (grade D).

W11: The new saturation (and the new delivery system) and flow rate should be recorded on the patient's observation chart after $5 \mathrm{~min}$ of treatment at the new oxygen concentration. Each change should be recorded by the clinician trained to administer oxygen by signing the observation chart (only changes should be signed for) (grade D).

W12: Repeat blood gas measurements are not required for stable patients who require a reduced concentration of oxygen (or cessation of oxygen therapy) to maintain the desired target saturation (grade D).

W13: Patients with no risk of hypercapnic respiratory failure do not always need repeat blood gas measurements after an increase in oxygen concentration. However, the patient requires clinical review to determine why the oxygen saturation has fallen (grade D).

W14: Patients at risk of hypercapnic respiratory failure (usually those with a target range of $88-92 \%$; see table 4 ) require repeat blood gas assessment 30-60 min after an increase in oxygen therapy (to ensure that the carbon dioxide level is not rising) (grade D).

W15: For patients with no risk of hypercapnic respiratory failure, monitoring by pulse oximeter is sufficient (repeated blood gases not required) provided the patient is clinically stable and the oxygen saturation remains in the desired range, usually 94-98\% (grade D).

W16: If a patient's oxygen saturation is lower than the prescribed target range, first check all aspects of the oxygen delivery system and the oximeter device for faults or errors (grade D).

W17: If a patient's oxygen saturation is consistently lower than the prescribed target range, there should be a medical review and the oxygen therapy should be increased according to an agreed written protocol (grade D).

W18: If the oxygen saturation fails to rise following 5-10 min of increased oxygen therapy or if there is clinical concern following medical review, then blood gas measurements should be repeated (grade D).

\section{$X$ Training in oxygen prescribing and use}

X1: All clinicians prescribing oxygen should have appropriate training and access to written or electronic oxygen prescribing guidelines based on this national guideline (grade D).

(Training slides for doctors and nurses are available as online appendices 7 and 8 on the BTS website.)

$X 2$ : Every hospital should have a training programme to ensure that clinical staff are familiar with the hospital's oxygen administration policies. In view of the high number of adverse incidents related to oxygen therapy, it is recommended that all acute Trusts should include basic training in oxygen use in the mandatory training programmes for all clinical staff (grade D). 


\section{SECTION 1: INTRODUCTION}

\subsection{Aim of the guideline}

The key aim of this guideline is to make oxygen use in emergency and healthcare settings safer, simpler and more effective. Oxygen is probably the commonest drug used in the care of patients who present with medical emergencies. Prior to the publication of the first BTS guideline for emergency oxygen use in adult patients in $2008,{ }^{2}$ ambulance teams and emergency department teams were likely to give oxygen to virtually all breathless or seriously ill patients and also to a large number of non-hypoxaemic patients with conditions such as ischaemic heart disease or stroke based on custom and practice. About 34\% of UK ambulance journeys in 2007 involved oxygen use. ${ }^{3}$ This translated to about two million instances of emergency oxygen use per annum by all UK ambulance services, with further use in patients' homes, GP surgeries and in hospitals. Audits of oxygen use and oxygen prescription have shown consistently poor performance in many countries and most clinicians who deal with medical emergencies have encountered adverse incidents and occasional deaths due to underuse and overuse of oxygen. ${ }^{4-10}$

Historically, oxygen has been administered for three main indications of which only one is evidence-based. First, oxygen is given to correct hypoxaemia because severe hypoxaemia is clearly harmful to the human body. Second, oxygen has been administered to ill patients in case they might become hypoxaemic. Recent evidence suggests that this practice may actually place patients at increased risk if impaired gas exchange does actually develop (see section 6.3). Third, a very high proportion of medical oxygen was administered because most clinicians believed, prior to 2008, that oxygen can alleviate breathlessness in most circumstances. However, there is no good evidence that oxygen relieves breathlessness in nonhypoxaemic patients. There is evidence of lack of effectiveness or minimal effectiveness in mildly hypoxaemic breathless patients with COPD and advanced cancer (see sections 6 and 8.11.4).

Against this background, the Standards of Care Committee of the BTS established a working party in association with 21 other societies to produce an evidence-based guideline for emergency oxygen use in the UK. This led to the production of the 2008 BTS guideline for emergency oxygen use in adult patients which was the world's first guideline for emergency oxygen therapy. ${ }^{2}$ This guideline has been implemented throughout the UK and in many other countries leading to over 500 citations in the medical literature up to the end of 2016.

The purpose of the update to the 2008 guideline is to strengthen the evidence base of the previous guideline based on revised methodology (which meets criteria contained in the AGREE II instrument) and to extend the evidence base to the end of 2013. ${ }^{11}$ Additionally, the remit of the 2008 guideline has been broadened to cover several new aspects of oxygen use and a broader range of locations where oxygen might be used.

\subsection{Intended users of the guideline and target patient populations}

This guideline is mainly intended for use by all healthcare professionals who may be involved in emergency oxygen use. This will include ambulance staff, first responders, paramedics, doctors, nurses, midwives, physiotherapists, pharmacists and all other healthcare professionals who may deal with ill or breathless patients. Advice is also provided for first responders belonging to voluntary organisations or other non-National Health Service (NHS) bodies. Information based on this guideline is available on the BTS website for use in the following situations:

- Hospital use

- Primary care use
- Ambulance use (supplemented by ambulance service guidance based on this guideline) ${ }^{12}$

- Use by nursing staff and allied health professions.

These abbreviated versions of the guideline contain the key recommendations and tables and charts that are relevant to the particular situation. The 'mini guidelines' can be downloaded by healthcare organisations for use on Trust intranets and to produce paper versions of the guideline for key staff.

\subsection{Areas covered by this guideline}

The guideline addresses the use of oxygen in three main categories of adult patients in the prehospital and hospital setting and in other settings such as palliative care:

- Critically ill patients,

- Hypoxaemic patients and patients at risk of hypoxaemia,

- Non-hypoxaemic patients who may benefit from oxygen (eg, carbon monoxide poisoning).

\subsection{Areas not covered by this guideline}

- Oxygen use in paediatrics: the present guideline applies only to patients aged $>16$ years.

- Oxygen use for high altitude activities.

- Oxygen use during air travel.

- Underwater diving and diving accidents.

- Oxygen use in animal experiments.

- Oxygen use in HDUs.

- Oxygen use in ICUs.

- Interhospital level 3 transfers.

- Hyperbaric oxygen.

- Respiratory support techniques including tracheal intubation, invasive ventilation and NIV (CPAP is included).

- Self-initiated use of oxygen by patients who have home oxygen for any reason.

- Ongoing care of hypoxaemic patients at home.

\subsection{Key changes since the first edition of this guideline published in 2008 \\ Methodology}

The evidence review methodology has changed from NICE methodology to the BTS NICE accredited guideline production process which is based on the Scottish Intercollegiate Guideline Network (SIGN) methodology and adheres to AGREE methodology (see section 1).

Evidence levels and grade of recommendation

These are now in SIGN format (see section 1 and tables 6 and 7).

\section{Evidence base}

The evidence base for the guideline has been updated to August 2013 (and extended to late-2016 for key references). None of the 2008 recommendations have been challenged by new evidence but many of the existing recommendations are supported by new information. There have been many observational studies but few randomised trials directly relevant to the guideline since 2008 .

The remit of the guideline has been extended

The new guideline covers not just emergency oxygen use but most oxygen use in healthcare settings. It also covers short-term oxygen use by healthcare workers outside of healthcare settings but domiciliary oxygen use by patients is covered by the BTS guideline for home oxygen use in adults. ${ }^{13}$ 
Table 6 SIGN levels of evidence ${ }^{14}$

\begin{tabular}{|c|c|}
\hline $\begin{array}{l}\text { SIGN levels of } \\
\text { evidence }\end{array}$ & \\
\hline $1++$ & $\begin{array}{l}\text { High-quality meta-analyses, systematic reviews of RCTs, or } \\
\text { RCTs with a very low risk of bias }\end{array}$ \\
\hline $1+$ & $\begin{array}{l}\text { Well-conducted meta-analyses, systematic reviews or RCTs } \\
\text { with a low risk of bias }\end{array}$ \\
\hline 1 & $\begin{array}{l}\text { Meta-analyses, systematic reviews, or RCTs with a high } \\
\text { risk of bias }\end{array}$ \\
\hline $2++$ & $\begin{array}{l}\text { High-quality systematic reviews of case-control or cohort } \\
\text { studies } \\
\text { High-quality case-control or cohort studies with a very } \\
\text { low risk of confounding or bias and a high probability } \\
\text { that the relationship is causal }\end{array}$ \\
\hline $2+$ & $\begin{array}{l}\text { Well-conducted case-control or cohort studies with a low } \\
\text { risk of confounding or bias and a moderate probability } \\
\text { that the relationship is causal }\end{array}$ \\
\hline $2-$ & $\begin{array}{l}\text { Case-control or cohort studies with a high risk of } \\
\text { confounding or bias and a significant risk that the } \\
\text { relationship is not causal }\end{array}$ \\
\hline 3 & Non-analytic studies, for example, case reports, case series \\
\hline 4 & Expert opinion \\
\hline
\end{tabular}

Table 7 SIGN grades of recommendation

\section{Grades of recommendations}

A At least one meta-analysis, systematic review or RCT rated as $1++$, and directly applicable to the target population; or

A body of evidence consisting principally of studies rated as $1+$, directly applicable to the target population, and demonstrating overall consistency of results

B A body of evidence including studies rated as $2++$, directly applicable to the target population, and demonstrating overall consistency of results; or Extrapolated evidence from studies rated as $1++$ or $1+$

C A body of evidence including studies rated as $2+$, directly applicable to the target population and demonstrating overall consistency of results; or Extrapolated evidence from studies rated as $2++$

D Evidence level 3 or 4; or Extrapolated evidence from studies rated as $2+$

Good practice points

$\checkmark$ Recommended best practice based on the clinical experience of the Guideline Development Group

RCT, randomised controlled trial; SIGN, Scottish Intercollegiate Guideline Network.

The scope of the guideline has been widened

The present guideline includes the following new topics and settings which have been requested by guideline users:

- Postoperative and perioperative care including PCA,

- Endoscopy and other procedures requiring conscious sedation,

- Palliative care settings including hospices,

- Use of helium-oxygen mixtures (Heliox) and nitrous oxide/ oxygen mixtures (Entonox),

- Use of CPAP,

- Use of oxygen by healthcare professionals in patients' homes,

- Use of oxygen by voluntary rescue organisations and other non-NHS first responders,

- High-flow nasal cannulae (HFNC).

The structure and format of the guideline has been changed since 2008:

The 2008 guideline was published as a supplement in Thorax. ${ }^{2}$ Additional educational materials and other resources including audit tools were made available on the BTS website. The new guideline exists in two complementary formats.
- A concise guideline which contains recommendations and good practice points is published in BMJ Open Respiratory Research.

- The full guideline including evidence review, physiology overview, illustrations and references is published in this edition of Thorax and is available on the BTS website http:// www.brit-thoracic.org.uk.

\subsection{Limitations of the guideline}

This guideline is based on the best available evidence concerning oxygen therapy. However, a guideline can never be a substitute for clinical judgement in individual cases. There may be cases where it is appropriate for clinicians to act outwith the advice contained in this guideline because of the needs of individual patients, especially those with complex or interacting disease states. Furthermore, the responsibility for the care of individual patients rests with the clinician in charge of the patient's care and the advice offered in this guideline must, of necessity, be of a general nature and should not be relied on as the only source of advice in the treatment of individual patients. In particular, this guideline gives very little advice about the management of the many medical conditions that may cause hypoxaemia (apart from the specific issue of managing the patients' hypoxaemia). Readers are referred to other guidelines for advice on the management of specific conditions such as COPD, pneumonia, heart failure, etc. Some of these disease-specific guidelines may suggest slightly different approaches to emergency oxygen therapy whereas the present guideline aims to provide simple all-embracing advice about oxygen therapy.

\section{SECTION 2: METHODOLOGY OF GUIDELINE PRODUCTION \\ 2.1 Establishment of guideline team}

The need for a national guideline for emergency oxygen use was recognised by the BTS Standards of Care Committee in 2003. A working party was established with representatives from a wide range of professions involved in oxygen therapy and a lay representative. The original group was expanded in 2006 because it became clear that the development and implementation of a national guideline would require input from a very wide range of professional groups. This group agreed the remit of the 2008 guideline and a series of key questions which were addressed within the 2008 guideline. ${ }^{2}$ The group membership was expanded further and the remit was expanded for the 2016 update of the guideline. A full list of guideline group members is provided in annex 1. The methodology for the 2016 guideline adheres to the BTS Guideline Production Manual 2014 which is aligned to the AGREE criteria for guideline production. ${ }^{11} 14$

A MEDLINE search for 'oxygen' yielded over a quarter of a million 'hits', most of which were not relevant to this guideline. For this reason, the BTS commissioned the Centre for Reviews and Dissemination and Centre for Health Economics at the University of York to undertake bespoke literature searches based on the literature search strategies employed for the 2008 guideline. The search strategies are shown in detail in online supplementary appendix 1 on the BTS website (http://www. brit-thoracic.org.uk).

\subsection{Summary of key questions}

Key question 1: physiology and pathophysiology of oxygen

- What are the dangers of hypoxia/hypoxaemia (ie, what happens to the human body)?

- What level of hypoxaemia is dangerous to all patients (even healthy adults)? 
- What level of hypoxaemia is dangerous to vulnerable groups (eg, ischaemic heart disease, stroke, elderly)?

- Repeat the above searches with additional key words: elderly, stroke, myocardial infarction, heart failure, COPD, trauma, renal failure.

- Same questions for hypercarbia/hypercapnia:

- Search for 'hypercapnia' combined with terms implying a harmful outcome (death/tissue injury/brain damage/coma).

- What level of hypercapnia is dangerous to all patients?

- What level of hypercapnia is dangerous to vulnerable groups (as above)?

- Same questions for respiratory acidosis:

- Search for 'respiratory acidosis' combined with terms implying a harmful outcome (death/tissue injury/brain damage/coma).

- What level of respiratory acidosis is dangerous to all patients?

- What level of respiratory acidosis is dangerous to vulnerable groups (as above)?

Key question 2: clinical aspects of hypoxaemia and oxygen therapy for common medical emergencies

- How to assess hypoxaemia (clinical, EWS systems, oximetry, arterial and capillary blood gases).

- How to assess hypercarbia/hypercapnia.

- Use of oxygen to relieve symptomatic breathlessness.

- Use of oxygen in acute COPD.

- Use of oxygen in acute asthma.

- Use of oxygen in pneumonia.

- Use of oxygen for pulmonary embolus.

- Use of oxygen in trauma.

- Use of oxygen in heart failure.

- Use of oxygen in myocardial infarction and unstable coronary artery syndrome.

- Use of oxygen in angina.

- Use of oxygen for other patients with less common conditions were searched individually (eg, CF, muscular dystrophy, motor neurone disease, severe kyphoscoliosis, anaphylaxis, hyperventilation).

Key question 3: oxygen prescription, oxygen delivery systems and oxygen transport

- Oxygen carriage in transport (practical issues; safety issues).

- Oxygen delivery systems in ambulances.

- Prescription of oxygen.

- Local hospital guidelines for oxygen use.

- Oxygen delivery systems in hospitals and other healthcare and emergency settings.

- Advantages/disadvantages of each delivery system (Venturi masks, simple face masks, nasal cannulae, high-flow masks such as non-rebreathing reservoir masks). Use of oxygendriven nebulisers.

- Use of CPAP.

- Use of 'alert cards', alert bracelets or similar hazard warning systems for patients who are known to be at risk of hypercapnia.

\subsection{How the evidence was assimilated into the guideline}

The search strategy and guideline methodology for the 2008 guideline are described within the original guideline. ${ }^{2}$ The remit of the guideline was widened for this update. Significant new areas include the use of oxygen during conscious sedation procedures, the non-emergency use of oxygen in healthcare settings and the use of CPAP, Heliox and oxygen-nitrous oxide mixtures. The methodology used for the current guideline was based on SIGN methodology as outlined in the BTS Guideline Production Manual 2014. ${ }^{11} 1415$

The 2008 guideline had used NICE levels of evidence so all searches were rerun in November 2011 and again in August 2013. All abstracts retrieved by the literature search were screened by pairs of members and reprints of all relevant papers were obtained. Members of the Guideline Development Group worked in pairs to assign a SIGN level of evidence to all of the papers that were judged to be relevant to the guideline (see tables 6 and 7). Further references were obtained from the group's personal literature collections and from the references contained within the papers which the search yielded and by focused literature searches by members of the guideline group. The group continued to monitor the literature up to the end of 2016 for important new publications or very high-quality abstracts from international meetings that were thought to be relevant to this guideline.

The group was divided into subgroups to work on each chapter of the guideline. Contributions by each member of the group are acknowledged in annex 1. The Guideline Development Group corresponded by email on a regular basis to discuss the evidence and to update the guideline and its key recommendations over the course of 2011-2016. Oxygen therapy is unusual insofar as there are very few published trials where different levels of oxygen therapy have been compared in randomised studies which reported clinically relevant outcomes. Most advice concerning oxygen therapy is based on expert opinion guided by extrapolation from non-interventional studies that do not directly address guideline questions. For this reason, most of the recommendations in this guideline are at grade D and it is hoped that the deficiency of relevant evidence will stimulate researchers to conduct randomised trials of oxygen therapy. However, the fact that a recommendation is graded as 'grade D' due to lack of evidence does not imply that the recommendation is not important of that there is any uncertainty as to the correct course of action. For example, it would never be ethical to undertake a randomised controlled trial (RCT) of oxygen therapy in severe hypoxaemia, so the advice to use oxygen to correct severe hypoxaemia will always be a grade D recommendation but it is one of the most important recommendations in this guideline.

Meetings of the full group were held in November 2011 and September 2012. The updated guideline was reviewed by the BTS Standards of Care Committee in September 2014, March 2015, September 2015 and June 2016. The guideline was further refined by email discussion following comments by this committee. The draft was made available via the BTS website for 6 weeks from 7 December 2015 to 18 January 2016 for public and stakeholder consultation and comments were invited. The draft document was sent to two peer reviewers at that time. The revised document was then submitted to the British Thoracic Society for final approval in October 2016 and endorsement invited from the other stakeholder societies and colleges.

\subsection{Piloting, implementing and auditing the guideline}

The principles of the 2008 guideline (target saturation ranges, etc) were piloted at Salford Royal University Hospital and Southend University Hospital prior to UK-wide dissemination in 2008. A baseline audit of oxygen use in UK hospitals was undertaken in 2008 prior to the launch of the guideline and the process has been audited annually by the BTS each subsequent year, these audits have monitored implementation of guideline policies in addition to an audit of process at each hospital. ${ }^{16}$ 
Key features of successful policy implementation were:

- Trust-wide introduction of the agreed hospital policy.

- Each hospital in the UK has one or more 'oxygen champions' to implement and audit the safe use of oxygen locally (see section 14.6).

- Local induction and educational programme for doctors, nurses and other users of oxygen.

- Introduction of prescription charts and patient observation charts to facilitate the standardisation of oxygen therapy (figure 19). Production and implementation of a detailed hospital oxygen policy based on a model document on the BTS website (see online supplementary appendix).

- Use of standard charts to guide the prescription and administration of oxygen (figure 19).

The educational materials and lecture presentations in web appendices 7-11 have been used widely in the UK with feedback from the oxygen champions in each hospital.

\subsection{Planned review and updating of the guideline}

The guideline will be reviewed by the British Thoracic Society within 5 years from publication.

\subsection{Declarations of interest}

All members of the guideline group made declarations of interest in line with BTS policy and further details can be obtained on request from BTS.

\section{SECTION 3: NORMAL VALUES AND DEFINITIONS}

- Normal blood levels of oxygen and carbon dioxide.

- Normal oxygen saturation $\left(\mathrm{SaO}_{2}\right)$ and normal blood $\mathrm{pH}$.

- Definitions of hypoxaemia, hypoxia, hypercapnia, acidosis, respiratory failure.

Oxygen is transported in the blood in two forms: a small and negligible amount is dissolved in the plasma and the majority is bound to haemoglobin and delivered to the tissues. Oxygen demand and oxygen delivery increase during exercise and reduce during rest and sleep.

\subsection{Blood gas levels of oxygen and carbon dioxide in health and disease}

The human lung delivers oxygen to the blood and removes carbon dioxide. Several mechanisms exist to regulate breathing in such a way that both gases are maintained within quite a narrow range, although carbon dioxide levels are more tightly regulated than those of oxygen.

\subsubsection{Normal ranges for oxygen saturation $\left(\mathrm{SaO}_{2}\right.$ and $\left.\mathrm{SpO}_{2}\right)$ and} $\mathrm{PO}_{2}\left(\mathrm{PaO}_{2}\right)$ in the blood at sea level

As there is a fixed amount of haemoglobin circulating in the blood, the amount of oxygen carried in the blood is often expressed in terms of how saturated with oxygen the circulating haemoglobin is. This is what is meant by 'oxygen saturation level'. If this is measured directly from an arterial blood sample, it is termed $\mathrm{SaO}_{2}$. If it is measured from a pulse oximeter it is termed $\mathrm{SpO}_{2}$. Alternatively, one can measure the $\mathrm{PO}_{2}$ of the blood $\left(\mathrm{PaO}_{2}\right)$, known as the 'partial pressure of oxygen' in the blood. This measurement can be expressed in kilopascals ( $\mathrm{kPa}$; normal range $12.0-14.6 \mathrm{kPa}$ ) or in millimetres of mercury (normal range $90-110 \mathrm{~mm} \mathrm{Hg}$ for young adults). ${ }^{17}$ The precise normal ranges for oxygen saturation and $\mathrm{PO}_{2}$ are difficult to determine accurately due to a lack of data from the 'normal' population, that is, non-hospitalised healthy participants.

A US observational study of nearly 900 asymptomatic patients in the emergency department showed the median $\mathrm{SpO}_{2}$ value to be $99 \%$ with $\mathrm{SpO}_{2}$ values $<97 \%$ occurring in $5.7 \%$ of the study group. ${ }^{18}$ However, the study group were young (median age 38) and predominantly African-American, so these data are not generalisable to the UK population.

Another North American study measured blood gases and $\mathrm{SpO}_{2}$ at sea level in 96 healthy individuals aged between 18 and $81 .{ }^{17}$ They found that for adults 2SD range for $\mathrm{SaO}_{2}$ is $\sim 94-98 \%$ at sea level but this may decline gradually with advancing age (table 8 ).

A much larger observational study of over 37000 patients admitted to four acute medical admissions units across the $\mathrm{UK}^{19}$ found that median $\mathrm{SpO}_{2}$ was $98 \%$ for young adults aged 18 64 years (IQR 97-99\%). For adults aged $\geq 65$ years the median saturation was 96\% with IQR 95-98\% (table 9). While the authors of this study recommend setting a normal range of 96$98 \%$, their study excluded nearly $20 \%$ of patients who were receiving oxygen, many whom were likely to have a $\mathrm{SpO}_{2}$ in the lower end of the normal range. In further considering these study results, it is of note that the $\mathrm{PaO}_{2}$ is $0.8 \mathrm{kPa}(6 \mathrm{~mm} \mathrm{Hg})$ lower in the supine position than in the upright position and most emergency measurements are made in the supine position. ${ }^{20}$

\subsubsection{Oxygen saturation in older adults}

The mean $\mathrm{SaO}_{2}$ may be lower in older people than in young adults. However, it is difficult to dissociate the effects of advancing age from the effects of the diseases that become commoner in old age. Some papers have reported a fall in the blood $\mathrm{PaO}_{2}$

Table 8 Mean (SD) $\mathrm{PaO}_{2}(\mathrm{kPa}$ and $\mathrm{mm} \mathrm{Hg})$ and $\mathrm{SaO}_{2}(\%)$ values (with range)

Mean (SD) $\mathrm{PaO}_{2}$ and $\mathrm{SaO}_{2}$ values (with range) in $\mathrm{kPa}$ and $\mathrm{mm} \mathrm{Hg}$

\begin{tabular}{|c|c|c|c|c|}
\hline Age & $\begin{array}{l}\text { Mean (SD) } \mathrm{PaO}_{2} \\
\text { (kPa and } \mathrm{mm} \mathrm{Hg} \text { ) }\end{array}$ & $\begin{array}{l}\text { Range } \pm 2 \mathrm{SD} \mathrm{PaO}_{2} \\
(\mathrm{kPa} \text { and } \mathrm{mm} \mathrm{Hg})\end{array}$ & $\begin{array}{l}\text { Mean (SD) } \\
\mathrm{SaO}_{2}(\%)\end{array}$ & $\mathrm{SaO}_{2} \pm 2 \mathrm{SD}$ \\
\hline $18-24$ & $\begin{array}{l}13.4(0.71) \\
99.9(5.3)\end{array}$ & $\begin{array}{l}11.98-14.82 \\
89.3-110.5\end{array}$ & $96.9(0.40$ & $96.1-97.7$ \\
\hline $25-34$ & $\begin{array}{l}13.4(0.66) \\
99.8(4.9)\end{array}$ & $\begin{array}{l}12.08-14.72 \\
90-109.6\end{array}$ & $96.7(0.7)$ & $95.3-98.1$ \\
\hline 35-44 & $\begin{array}{c}13.18(1.02) \\
98.3(7.6)\end{array}$ & $\begin{array}{l}11.14-15.22 \\
83.1-113.5\end{array}$ & $96.7(0.6)$ & $95.5-97.9$ \\
\hline $45-54$ & $\begin{array}{c}13.0(1.07) \\
97(8)\end{array}$ & $\begin{array}{l}10.86-15.14 \\
81-113\end{array}$ & 96.5 (1) & $94.4-98.5$ \\
\hline 55-64 & $\begin{array}{c}12.09(0.60) \\
90.2(4.5)\end{array}$ & $\begin{array}{l}10.89-13.29 \\
81.2-99.2\end{array}$ & $95.1(0.7)$ & $94.5-97.3$ \\
\hline$>64$ & $\begin{array}{r}11.89(1.43) \\
88.7(10.7)\end{array}$ & $\begin{array}{l}9.02-14.76 \\
67.3-110.1\end{array}$ & $95.5(1.4)$ & $92.7-98.3$ \\
\hline
\end{tabular}

Table 9 Ranges, mean, SD, median and IQR values for $\mathrm{SpO}_{2}(\%)$ where measurements were made with the patient receiving air for age $\geq 18$ years $(n=37299)$ from Smith et $a l^{19}$

\begin{tabular}{|c|c|c|c|c|c|c|c|}
\hline \multirow{2}{*}{$\begin{array}{l}\text { Age } \\
\text { (years) }\end{array}$} & \multirow[b]{2}{*}{$\mathrm{n}$} & \multicolumn{6}{|l|}{$\mathrm{SpO}_{2}$} \\
\hline & & Minimum & Maximum & Mean & SD & Median & IQR \\
\hline $18-24$ & 1663 & 80 & 100 & 98.0 & 1.8 & 98 & $97-99$ \\
\hline $25-34$ & 2239 & 82 & 100 & 97.6 & 1.9 & 98 & $97-99$ \\
\hline $35-44$ & 3412 & 73 & 100 & 97.2 & 2.1 & 98 & $96-99$ \\
\hline $45-54$ & 4103 & 76 & 100 & 96.8 & 2.2 & 97 & $96-98$ \\
\hline $55-64$ & 5020 & 72 & 100 & 96.3 & 2.5 & 97 & 95-98 \\
\hline$\geq 65$ & 20862 & 70 & 100 & 95.8 & 2.7 & 96 & $95-98$ \\
\hline
\end{tabular}


in older participants. Indeed a fall in $\mathrm{SpO}_{2}$ in patients $>65$ was demonstrated in Crapo and Smith's studies, and the former study shows a decreasing $\mathrm{PaO}_{2}$ with age. However, others have failed to confirm this observation. ${ }^{21-23}$ The mean $\mathrm{SaO}_{2}$ in seated adults aged $>64$ years in one published study was $95.5 \%$ compared with $96.9 \%$ in adults aged $18-24$ years, and the SD was wider in the older age group with a $2 \mathrm{SD}$ range of 92.798.3\% (tables 8 and 9). ${ }^{17}$ The mean (SD) $\mathrm{SaO}_{2}$ for recumbent healthy men aged $>70$ years in another study was $95.3 \%$ (1.4\%) giving a 2SD range of $92.5-98.1 \%$ for men of this age. ${ }^{21}$ The mean (SD) $\mathrm{SaO}_{2}$ was $94.8 \%$ (1.7\%) for recumbent healthy women aged $>70$ years with a $2 \mathrm{SD}$ range of 91.5-98.2\%. The authors of this study did not observe any age-related decline in $\mathrm{SaO}_{2}$ beyond the age of 70 years. The mean $\mathrm{SaO}_{2}$ in this study of $\sim 95.0 \%$ for recumbent healthy men and women aged $>70$ years was below the normal range for seated healthy young adults. The mean $\mathrm{PaO}_{2}$ in older participants in this study was $10.3 \mathrm{kPa}$ for men and $9.8 \mathrm{kPa}$ for women, which is lower than two other studies which reported mean $\mathrm{PaO}_{2}$ values of $11.2 \mathrm{kPa}$ and $11.1 \mathrm{kPa}$ in healthy older participants. $^{22} 24$ Some of these differences are probably due to participant selection, but there may also be variations in the results obtained by different blood gas analysers. ${ }^{25}$

Further data from an unpublished audit of 320 stable hospital patients in Salford and Southend with no history of lung disease found a mean (SD) $\mathrm{SpO}_{2}$ of $96.7 \%$ (1.77\%; 2SD range 95.2$100 \%$ ) in patients aged $>71$ years ( $\mathrm{R}$ O'Driscoll, A Davison, $\mathrm{L}$ Ward, personal communication). These values were measured by pulse oximetry in UK hospitals in 2008 and are more likely to represent the expected normal range of pulse oximetry measurements in the elderly UK population. The variation with age, sex and posture makes it difficult to give a precise target range that will apply to all adults who might require oxygen therapy, but the guideline development committee believe that a target range of 94-98\% will achieve normal or near-normal $\mathrm{SpO}_{2} /$ $\mathrm{SaO}_{2}$ for most adults in the UK and will avoid any risk of clinically significant hypoxaemia.

\subsubsection{Oxygen saturation at altitude}

While the percentage of oxygen in inspired air is constant at 21\% and does not change with altitude, the fall in atmospheric pressure at higher altitudes decreases the partial pressure of inspired oxygen. The $\mathrm{SaO}_{2}$ at a given altitude varies with age, sex, ethnic group and degree of acclimatisation to altitude. For example, a sample of 3812 people of all ages living in Tibet at an altitude of about $4000 \mathrm{~m}$ had a mean $\mathrm{SaO}_{2}$ of only $88.2 \%$, but people native to the Andes had an $\mathrm{SaO}_{2}$ about $2.6 \%$ higher than Tibetans living at the same altitude. ${ }^{26} 27$ However, the physiology of hypoxaemia at altitude is very different to the physiology of hypoxaemia at sea level, for example, ventilator changes usually lead to a marked fall in arterial $\mathrm{CO}_{2}$ level. Millions of people live at these altitudes with $\mathrm{SaO}_{2}$ values that would cause concern at sea level. The city of $\mathrm{La} \mathrm{Paz}$ in Bolivia has a mean altitude of $3600 \mathrm{~m}$ and a population of $\sim 1.5$ million people. The $\mathrm{SaO}_{2}$ of climbers on Mount Everest $(8848 \mathrm{~m})$ can fall below $55 \% .^{28}$ Sudden exposure to altitudes above about $4000 \mathrm{~m}$ can cause mountain sickness, high altitude pulmonary oedema and high altitude cerebral oedema in unacclimatised individuals. Long-term exposure to high altitude (or to hypoxaemia for any other reason) can lead to pulmonary hypertension.

\subsubsection{0xygen saturation in acute and chronic diseases}

If the blood oxygen level falls to extremely low levels for even a few minutes (eg, during cardiac arrest), tissue hypoxia and cell death will occur, especially in the brain. The brain appears to be the most vulnerable organ during profound hypoxaemia; brain malfunction is the first symptom of hypoxia and brain injury is the most common long-term complication in survivors of cardiac arrests and other episodes of profound hypoxaemia. Sudden exposure to low $\mathrm{SaO}_{2}$ below about $80 \%$ can cause altered consciousness even in healthy participants. It is likely that other organs in patients with critical illness or chronic organ damage are vulnerable to the risk of hypoxic tissue injury at oxygen levels above $80 \%$.

Most experts emphasise the importance of keeping the $\mathrm{SaO}_{2}$ above $90 \%$ for most acutely ill patients. ${ }^{29-32}$ However, the degree of hypoxia that will cause cellular damage is not well established and probably is not an absolute value. Healthy older adults, for instance, have lower $\mathrm{SaO}_{2}$ values at rest than younger adults. Many patients with chronic lung disease, congenital cyanotic heart disease or chronic neuromuscular conditions have oxygen saturations substantially below the normal range, even when clinically stable.

However, although chronically hypoxaemic patients may tolerate an abnormally low $\mathrm{SaO}_{2}$ at rest when in a clinically stable condition, these resting oxygen levels may not be adequate for tissue oxygenation during acute illness when the tissue oxygen demand may increase (eg, sepsis, trauma, pneumonia, head injury; see section 8).

Acute hypoxaemia with $\mathrm{SaO}_{2}<90 \%$ and sometimes $<80 \%$ is seen in many acute illnesses such as pneumonia and heart failure and it is likely that the clinical manifestations of hypoxaemia in illness would be similar to those of experimental hypoxaemia in hypobaric chambers (impaired mental function followed by loss of consciousness). However, the clinical manifestations of the illness itself make it difficult to identify which symptoms and signs are due to hypoxaemia. Pure hypoxaemia, as seen in hypobaric chambers and at altitude, does not seem to cause breathlessness in resting participants. Patients with chronic diseases such as COPD, lung fibrosis, neuromuscular disorders or congenital heart disease may routinely attend outpatient clinics with $\mathrm{SaO}_{2}$ levels well below 90\% even at a time when their disease is stable. In an emergency, a clinician who was not familiar with such a patient (when stable) might interpret the low saturation as having occurred acutely and aim to achieve an oxygen saturation that was well above the patient's usual oxygen saturation level. Many such patients would qualify for long-term oxygen therapy. The UK COPD guideline recommends a threshold of $7.3 \mathrm{kPa}(55 \mathrm{~mm} \mathrm{Hg})$ below which most patients with COPD will benefit from long-term oxygen therapy (equivalent to $\mathrm{a} \mathrm{SaO}_{2}$ of about $88-89 \%$ ) and an $\mathrm{PaO}_{2}$ threshold below $8.0 \mathrm{kPa}(60 \mathrm{~mm} \mathrm{Hg})$ for patients with established cor pulmonale and some other subgroups. ${ }^{1}$

\subsubsection{Variation in oxygen saturation during sleep}

Healthy participants in all age groups have greater variation in $\mathrm{SaO}_{2}$ when sleeping than while awake. A study of 330 people referred to a sleep laboratory with normal results of overnight polysomnography (patients with cranial facial or neurological abnormalities or previously diagnosed pulmonary disease were excluded) showed that desaturation routinely occurred with a mean (SD) minimum $\mathrm{SaO}_{2}$ or 'nadir' of 90.4\% (3.1\%) during the night (2SD range $84.2-96.6 \%) .{ }^{33}$ The mean (SD) overnight $\mathrm{SaO}_{2}$ 'nadir' was $89.3 \% \quad(2.8 \%)$ for participants aged $>60$ years. ${ }^{33}$ In this study, participants aged $20-30$ years spent $10 \%$ of the night with $\mathrm{SaO}_{2}$ levels below $94.8 \%$ and half the night below $96.3 \%$, and those aged 60 years spent $10 \%$ of the night below $92.8 \%$ and half the night below 95.1\%. 
Furthermore, the authors of this study excluded obese patients with any features of sleep apnoea or hypopnoea because these patients are known to desaturate to very low levels during sleep (often below 70\%). The variation in $\mathrm{SaO}_{2}$ during sleep is exaggerated by alcohol and by sedative drugs. This makes it difficult to evaluate a 'spot reading' of $\mathrm{SaO}_{2}$ on a sleeping participant. It is suggested that $\mathrm{SaO}_{2}$ measurements of sleeping participants should be interpreted with caution and ideally observed for a few minutes to see if the participant has got sustained hypoxaemia or just a transient normal 'nocturnal dip'.

Oxygen saturation in acute and chronic disease is discussed in section 3.1.4.

Evidence statements (see recommendations $A 1-A 2$ ):

- Normal daytime haemoglobin $\mathrm{SaO}_{2}$ is $96-98 \%$ in young adults in the seated position at sea level but the lower limit falls slightly with age and is $\sim 94-98 \%$ in adults aged $>70$ years (evidence $2+$ ).

- All participants have transient dips in oxygen saturation at night with a mean nadir of $90.4 \%$ (2SD range 84.2-96.6\%) in healthy participants in all age groups (evidence level 3).

\subsubsection{Normal range for arterial carbon dioxide tension}

The reference range for arterial carbon dioxide tension $\left(\mathrm{PaCO}_{2}\right)$ is $4.6-6.1 \mathrm{kPa}(34-46 \mathrm{~mm} \mathrm{Hg})$ for healthy adult men aged $18-$ 38 years. ${ }^{34}$ Although this study was undertaken in 1948 , it is consistent with the clinical experience of the guideline group members and with most modern reference values for $\mathrm{PaCO}_{2}$. Although different laboratories and textbooks give slightly different reference values, all are within $0.2 \mathrm{kPa}$ of the above reference range. Any value of $\mathrm{PaCO}_{2}$ of $>6.1 \mathrm{kPa}(45 \mathrm{~mm} \mathrm{Hg})$ should be considered abnormal, but values up to $6.7 \mathrm{kPa}$ $(50 \mathrm{~mm} \mathrm{Hg})$ may be obtained by breath holding. ${ }^{35}$

\subsection{Definitions of hypoxaemia, hypoxia, type 1 respiratory failure and hyperoxaemia \\ Hypoxaemia}

Hypoxaemia refers to low $\mathrm{PO}_{2}$ or partial pressure of oxygen $\left(\mathrm{PaO}_{2}\right)$ in the blood. For practical reasons, hypoxaemia can also be measured in relation to oxyhaemoglobin saturation. In adults the normal range is influenced by age and comorbidity and the normal ranges for healthy adults are given in section 3.1.1. The precise level at which a patient becomes hypoxaemic is debatable. One could argue that any saturation below the lower limit of normal constitutes hypoxaemia. Various authors have defined hypoxaemia as $\mathrm{SaO}_{2}$ of (1) <94\%; (2) <92\%; (3) <90\%; or (4) $\mathrm{PaO}_{2}<60 \mathrm{~mm} \mathrm{Hg}$ or $8 \mathrm{kPa}^{4}{ }^{46-38}$ Most authors who have studied this area have defined hypoxaemia as $\mathrm{PaO}_{2}<60 \mathrm{~mm} \mathrm{Hg}$ $(8 \mathrm{kPa})$ or $\mathrm{SaO}_{2}<90 \% .{ }^{39}$ There is no known risk of hypoxic tissue injury above this level and other guidelines on critical care set $90 \%$ as the minimum below which $\mathrm{SaO}_{2}$ should not be allowed to fall. $^{31} 32$

Hypoxia: Hypoxia occurs when oxygen supplies are insufficient to meet oxygen demands in a particular compartment (eg, alveolar or tissue hypoxia). Tissue hypoxia may be subdivided into four main causes: hypoxaemic, anaemic, stagnant or histotoxic. Oxygen therapy may only correct hypoxia due to hypoxaemia, other ways to improve oxygen delivery to the tissues need to be considered.

Hypoxaemic hypoxia: Hypoxaemic hypoxia (sometimes also referred to as hypoxic hypoxia) is present when the oxygen content in the blood is low due to reduced partial pressure of oxygen. This occurs naturally at altitude or occurs secondary to right-to-left shunts, ventilation-perfusion (V/Q) mismatch, alveolar hypoventilation or diffusion impairment.
Type 1 respiratory failure, which is defined as $\mathrm{PaO}_{2}<8 \mathrm{kPa}$ or $60 \mathrm{~mm} \mathrm{Hg}$ (equivalent to $\mathrm{SaO}_{2}$ of $\sim 90 \%$ ) with a normal or low $\mathrm{PaCO}_{2}$ level is due to hypoxaemic hypoxia.

Anaemic hypoxia: Anaemic hypoxia results from a reduced level of haemoglobin available for oxygen transport. Although the patient may not be hypoxaemic (with a normal $\mathrm{PaO}_{2}$ and $\mathrm{SpO}_{2}$ ), the reduced oxygen content of the blood may lead to tissue hypoxia. Carbon monoxide poisoning may also produce a form of anaemic hypoxia by impairing the ability of haemoglobin to bind oxygen, thereby reducing oxygen-carrying capacity.

Stagnant hypoxia: Stagnant hypoxia is a low level of oxygen in the tissues due to inadequate blood flow (either globally or regionally). This condition may occur in the extremities if a person is exposed to cold temperatures for prolonged periods of time and it is the cause of gangrene in tissue that is deprived of blood in severe peripheral vascular disease. Stagnant hypoxia may occur in low cardiac output states.

Histotoxic hypoxia: Histotoxic hypoxia is an inability of the tissues to use oxygen due to interruption of normal cellular metabolism. The best known example of this occurs during cyanide poisoning which impairs cytochrome function. It is increasingly thought that mitochondrial dysfunction may lead to decreased oxygen usage in sepsis despite adequate oxygen delivery. This has also been termed 'cytopathic dysoxia'. ${ }^{40}$

Hyperoxia and hyperoxaemia: Hyperoxia and hyperoxaemia are the counterparts to the above terms and in this guideline refer to high oxygen content in a compartment and high $\mathrm{PO}_{2}$ in the blood, respectively. As stated above, for practical purposes the $\mathrm{PO}_{2}$ in the blood is often measured as oxyhaemoglobin saturation. Furthermore, this guideline is centred on providing target saturations for various conditions, but it should be noted that above a $\mathrm{PaO}_{2}$ of $\sim 16 \mathrm{kPa}(120 \mathrm{~mm} \mathrm{Hg})$ the oxyhaemoglobin saturation will obviously not change from $100 \%$, yet the effects of further increases in $\mathrm{PaO}_{2}$ may be important in certain conditions such as COPD. This is discussed in further detail in sections 5 and 6.

\subsection{Definition of hypercapnia and type 2 respiratory failure} Hypercapnia is present when the $\mathrm{PaCO}_{2}$ is above the normal range of 4.6-6.1 kPa (34-46 mm Hg) and patients with hypercapnia are said to have type 2 respiratory failure even if the oxygen saturation is in the normal range. A study of 3524 blood gas samples in a single hospital found that $27 \%$ had type 2 respiratory failure whereas $6 \%$ of samples had type 1 respiratory failure. Forty-one per cent of these samples exhibited hyperoxia $\left(\mathrm{PaO}_{2}>16 \mathrm{kPa}\right){ }^{41}$ Exclusion of emergency department samples (many of which were venous) and exclusion of repeat samples (which are common in patients with COPD) left $8.5 \%$ of samples showing type 1 respiratory failure and $22.7 \%$ showing hypercapnia. ${ }^{42}$ Hypercapnia was commoner than pure hypoxaemia on surgical wards and critical care areas as well as on medical wards.

\subsection{Definition of acidosis (respiratory acidosis and metabolic acidosis)}

Acidosis: Acidity in any fluid is determined by the concentration of hydrogen ions $\left[\mathrm{H}^{+}\right]$, and this is normally regulated between 35 and $45 \mathrm{nmol} / \mathrm{L}$. Acidity is more often expressed in terms of $\mathrm{pH}$ where $\mathrm{pH}=-\log _{10}\left[\mathrm{H}^{+}\right]$. The normal $\mathrm{pH}$ range of the blood in humans is between 7.35 and 7.45 units. Acidosis is defined as a $\mathrm{pH}<7.35\left(\left[\mathrm{H}^{+}\right]>45 \mathrm{nmol} / \mathrm{L}\right)$ and alkalosis is defined as a $\mathrm{pH}>7.45\left(\left[\mathrm{H}^{+}\right]<35 \mathrm{nmol} / \mathrm{L}\right)$. Acidosis can be caused by respiratory or metabolic disorders. 
Respiratory acidosis

$$
\mathrm{CO}_{2}+\mathrm{H}_{2} \mathrm{O} \Leftrightarrow \mathrm{H}_{2} \mathrm{CO}_{3} \Leftrightarrow \mathrm{H}^{+}+\mathrm{HCO}_{3}
$$

Carbon dioxide $\left(\mathrm{CO}_{2}\right)$ can combine with water $\left(\mathrm{H}_{2} \mathrm{O}\right)$ to form carbonic acid $\left(\mathrm{H}_{2} \mathrm{CO}_{3}\right)$ in the blood which, in turn, dissociates to bicarbonate $\left(\mathrm{HCO}_{3}^{-}\right)$and a hydrogen ion $\left(\mathrm{H}^{+}\right)$. Acute respiratory acidosis occurs if the $\mathrm{pH}$ of the blood falls below 7.35 $\left(\left[\mathrm{H}^{+}\right]>45 \mathrm{nmol} / \mathrm{L}\right)$ in the presence of a raised $\mathrm{CO}_{2}$ level. If respiratory acidosis has been present for more than a few hours, the kidney retains bicarbonate to buffer the acidity of the blood and, over hours to days, this may be sufficient to produce a normal $\mathrm{pH}$. This situation (high $\mathrm{PaCO}_{2}$ with high bicarbonate and normal $\mathrm{pH}$ ) is known as 'compensated respiratory acidosis'. This situation is common in patients with chronic severe but stable COPD, but they may have an additional acute rise in $\mathrm{PaCO}_{2}$ during an acute exacerbation giving rise to 'acute on chronic' respiratory acidosis despite their high bicarbonate level. This happens because the bicarbonate level was equilibrated with the previous $\mathrm{CO}_{2}$ level and is insufficient to buffer the sudden further increase in $\mathrm{CO}_{2}$ level that may occur during an exacerbation of COPD. Respiratory acidosis is common in clinical practice. Plant et $a l^{43}$ showed that about $20 \%$ of patients with AECOPD requiring hospital admission have respiratory acidosis.

Metabolic acidosis: This can be caused by failure to excrete acid produced by the body's normal metabolic processes (eg, during renal failure) or by increased production of acid from abnormal metabolic conditions such as diabetic ketoacidosis. Alternatively, it may result from direct loss of bicarbonate from the kidney or gut (eg, during chronic diarrhoea). In all forms of metabolic acidosis, there is a low blood bicarbonate level, either due to loss of bicarbonate or due to buffering of excess acid by bicarbonate which is excreted as $\mathrm{CO}_{2}$. A common cause of metabolic acidosis is lactic acidosis caused by tissue hypoxia. This may result from decreased oxygen delivery such as occurs in hypoxaemia, or low cardiac output states or conditions such as sepsis where oxygen consumption is impaired in the face of adequate oxygen delivery. In health, metabolic acidosis will occur at peak exercise where oxygen delivery is insufficient to meet demand.

\section{SECTION 4: GENERAL BLOOD GAS PHYSIOLOGY}

A full understanding of blood gas physiology in the body requires a detailed understanding of the anatomy, physiology and biochemistry of respiration and gas exchange. It is recognised that most readers of this guideline may not have had full training in all of these specialties, so this physiology section contains a brief overview of basic principles for the non-specialist reader. The rationale for targeted oxygen therapy is discussed in detail in section 6 .

\subsection{Oxygen physiology}

Oxygen is transported in the blood in two forms: a small and negligible amount is dissolved in the plasma and the majority is bound to the haemoglobin molecule. As there is a fixed amount of haemoglobin circulating in the blood, the amount of oxygen carried in the blood is often expressed in terms of how saturated circulating haemoglobin is with oxygen $\left(\mathrm{SO}_{2}\right)$.

As discussed in section 3.1 the precise normal $\mathrm{SaO}_{2}$ in healthy adults at sea level is not known. However, it is within a narrow range of about 95-98\%. This means that almost all of the oxygen-carrying capacity of haemoglobin in the blood is used when the $\mathrm{SaO}_{2}$ is in the normal range. Therefore, giving supplemental oxygen to a healthy young person will increase the saturation level only slightly from about $97 \%$ to $99 \%$ or a maximum of $100 \%$, thus producing only a very small increase in the amount of oxygen made available to the tissues.

Sudden exposure to low $\mathrm{SaO}_{2}$ levels (below about 80\%) can cause impaired mental functioning even in healthy participants. The brain is the most sensitive organ to the adverse effects of hypoxia, but other organs in patients with critical illness may be vulnerable to the risk of hypoxic tissue injury at oxygen levels above this range. Most experts emphasise the importance of keeping the $\mathrm{SaO}_{2}$ above $90 \%$ for the majority of acutely ill patients. $^{29-32}$ The present guideline suggests a desirable target saturation range of $94-98 \%$. This range mirrors the normal range for UK adults with a wide margin of safety above the 90\% threshold aforementioned.

Oxygen passes from inspired air in the lungs into the bloodstream and is delivered to the tissues. If the $\mathrm{PaO}_{2}$ (partial pressure) falls in the blood, this is sensed by receptors in the carotid body (supplied by the carotid artery in the neck) and ventilation is stimulated to increase the amount of oxygen coming into the lung and therefore the blood. The lung has the ability to divert blood flow away from areas which are poorly ventilated, so that blood returning from the body can be efficiently replenished with oxygen and also cleared of carbon dioxide. This occurs through a process called 'hypoxic vasoconstriction' whereby localised low $\mathrm{PO}_{2}$ in the lung airspaces cause constriction of feeding blood vessels, therefore diverting blood to areas of the lung with better ventilation. This mechanism is unique to the lungs: the circulation of other organs such as the brain, heart and kidneys will vasodilate in response to hypoxia to facilitate greater blood flow to areas starved of oxygen.

If the oxygen-carrying capacity of the blood is low as, for example, in anaemia, this is detected by the kidneys which produce a hormone, erythropoietin, to stimulate red blood cell production; however, this process takes place over a course of days to weeks. As one of the goals of the circulation is to deliver oxygen to the tissues of the body, the heart also responds to low oxygen levels by increasing its output, so increasing 'oxygen delivery'. This takes place in seconds.

Hypoxaemia, low $\mathrm{PaO}_{2}$, can be caused by a number of mechanisms. It occurs naturally at altitude or can occur secondary to $\mathrm{V} / \mathrm{Q}$ mismatch, that is, as a result of poor aeration of areas of lung or due to abnormalities of gas exchange within the lung during serious illnesses such as pneumonia, where hypoxic vasoconstriction may not be able to compensate for this mismatch. This form of hypoxaemia is the easiest to treat with oxygen therapy. Oxygen therapy is less effective in other causes of tissue hypoxia including anaemia where there is a low carrying capacity or where the carrying capacity of haemoglobin has been reduced by a toxic substance since oxygen availability is not the limiting feature in these conditions. For example, carbon monoxide blocks oxygen binding to haemoglobin despite having a normal level of oxygen in the lungs and in the blood.

\subsection{Carbon dioxide physiology}

Carbon dioxide is a product of the body's metabolism. It is cleared from the body by being transferred from the bloodstream into the alveoli in the lungs and then exhaled from the lungs. It is also excreted via the kidneys where $\mathrm{CO}_{2}$ and water form carbonic acid which then dissociates into $\mathrm{H}^{+}$and $\mathrm{HCO}_{3}^{-}$. 
In a similar way to oxygen, carbon dioxide levels in the blood are controlled by chemical sensors (in the carotid body and brainstem).

Carbon dioxide is highly soluble in the blood and is carried in three forms: bicarbonate (70-85\%), dissolved carbon dioxide (5$10 \%)$ and bound to haemoglobin (10-20\%), the percentages varying according to whether it is arterial or venous blood. As carbon dioxide carriage is not limited by a carrier molecule such as haemoglobin, it is not expressed as a saturation. Because its carriage is approximately proportional to the partial pressure (gas tension) of carbon dioxide in the blood within the physiological range, carbon dioxide carriage is usually expressed in terms of its partial pressure. The normal range is $4.6-6.1 \mathrm{kPa}$ or $34-46 \mathrm{~mm} \mathrm{Hg}$.

Increased levels of carbon dioxide will stimulate ventilation, thus increasing clearance from the lungs and therefore from the bloodstream. However, this mechanism is less effective in some respiratory diseases such as COPD where increased airway resistance and respiratory muscle weakness can restrict this response, or where loss of the hypercapnic drive (eg, during chronic hypercapnia or severe brain injury) also depresses the ventilatory response. Hypercapnia will occur when there is decreased effective or 'alveolar' ventilation for any reason. Safe elimination of carbon dioxide is an important physiological process in the body to maintain $\mathrm{pH}$.

Too little oxygen can give rise to increased respiratory work to combat the hypoxaemia and, potentially, organ dysfunction and failure. However, too much oxygen can also be harmful in some situations especially to some vulnerable patients with COPD, chest wall deformities or muscle weakness.

Studies have shown that between $20 \%$ and $50 \%$ of patients with AECOPD or with obesity-hypoventilation syndrome (OHS) are at risk of carbon dioxide retention if they are given an excessively high concentration of oxygen. ${ }^{43-47}$ If high concentrations of oxygen are given to these patients, the oxygen level in the blood will rise but the level of carbon dioxide will also rise. This can cause acidosis and, when severe, coma. In the past it was thought that the main problem was that these patients were dependent on the stimulus of a low blood oxygen level-called 'hypoxic drive'-to stimulate breathing. It was thought that giving oxygen would cause a rise in the carbon dioxide level by simply reducing the stimulus to breathe due to 'lack of hypoxic drive'. However, studies have shown that the mechanisms for carbon dioxide retention are far more complex than this simple model suggests. ${ }^{48-50}$

Oxygen-induced hypercapnia in patients with AECOPD can be avoided by giving targeted lower concentration oxygen therapy to vulnerable patients and aiming for a target range of $88-92 \%{ }^{51}$ (see table 3 and recommendation A3).

\subsection{Concept of target oxygen saturation $\left(\mathrm{SaO}_{2}\right)$ ranges}

One might ask why one should not aim for a $\mathrm{SaO}_{2}$ of $100 \%$ (hyperoxaemia) in all acutely ill patients. This policy has been shown to worsen outcomes in vulnerable patients with COPD and chest wall problems, but there is also a potential for harm to other patients.

While the administration of oxygen to the hypoxaemic patient leads to an increase in $\mathrm{PaO}_{2}$ which leads to favourable physiological effects and ultimately the prevention of cell death, administering oxygen to the non-hypoxaemic patient has other physiological effects which are not widely appreciated, although how important these are clinically is not clear in most cases. These potentially adverse effects include direct pulmonary toxicity, coronary vasoconstriction, decreased cardiac output, increased free radical generation and the potential to delay the recognition of physiological deterioration due to the masking of any desaturation. These risks are discussed in detail in section 6 .

For this reason, the guideline group recognises the importance of a physiological target saturation range to guide staff to administer oxygen in a concentration to treat hypoxaemia without the risks of hyperoxaemia. As alluded to in section 3, some patients are chronically hypoxaemic and tolerate lower than normal saturations. The only RCT of oxygen therapy in patients with AECOPD randomised patients to receive highconcentration oxygen therapy versus titrated oxygen to keep $\mathrm{SpO}_{2} 88-92 \%$ in the prehospital setting. ${ }^{51}$ Mortality was significantly lower in patients receiving titrated oxygen rather than high-concentration oxygen. This study highlights the importance of maintaining a target saturation when administering oxygen to this and other high-risk groups.

Since the purpose of oxygen therapy is to increase oxygen delivery to tissues, not just to increase oxygen carried by the blood, it must be remembered that there may be other physiological disturbances that need correcting to increase oxygen delivery such as low cardiac output and severe anaemia. For example, improving these factors will improve oxygen delivery much more than administering oxygen to a patient with a saturation of $90 \%$ which, at most, will produce a $10 \%$ rise in delivery. In addition to optimising oxygen delivery from the lungs to the tissues $\left(\mathrm{DO}_{2}\right)$, it is important also to treat problems that might impair delivery of oxygen to the lungs themselves such as upper airway obstruction, bronchoconstriction and pulmonary oedema (remember the 'ABC' of resuscitation-airway, breathing, circulation).

Since the first publication of these guidelines, further studies have emerged investigating the role of oxygen therapy in diseases other than chronic lung disease. These have demonstrated the potential risks of hyperoxia and will be discussed in later sections. Despite these new data, there are still insufficient data to determine the exact target range in healthy adults. The consensus among the members of the guideline group is that one should aim for a normal or near-normal $\mathrm{SaO}_{2}$ range of $94-98 \%$ for acutely ill patients except those at risk of hypercapnic respiratory failure when a target saturation range of $88-92 \%$ should be used (see recommendations A1-A5).

\section{SECTION 5: ADVANCED BLOOD GAS PHYSIOLOGY}

This section is a more in-depth description of the physiological issues surrounding oxygen therapy. Recommendations $1-5$ in section 6 of the guideline will follow logically from this section and from the brief overview of oxygen physiology in section 4 .

\subsection{Oxygen delivery}

The neurocardiopulmonary axis is designed to optimise global oxygen delivery and carbon dioxide clearance and the local tissue vascular beds are responsible for the distribution of blood flow.

$\mathrm{DO}_{2}$ is expressed by the equation:

$$
\mathrm{DO}_{2}=\mathrm{CaO}_{2} \times \mathrm{Q}
$$

where $\mathrm{CaO}_{2}$ is the oxygen content of the arterial blood and $\mathrm{Q}$ is the cardiac output. $\mathrm{CaO}_{2}$ is the sum of oxygen dissolved in the blood and the amount of oxygen carried by haemoglobin. The solubility of oxygen in the blood is very low and therefore $\mathrm{CaO}_{2}$ is largely determined by the total amount of haemoglobin and the proportion which is bound to oxygen; which in turn is dependent on the haemoglobin per cent saturation. 
Figure 3 shows the level of oxygen and carbon dioxide in the pulmonary artery, in the alveolus and room air and in the pulmonary venous circulation which leads directly to the arterial circulation. The $\mathrm{PaO}_{2}$ of mixed systemic venous blood rises markedly from a low level in the pulmonary artery (about $6 \mathrm{kPa}$ or $45 \mathrm{~mm} \mathrm{Hg})$ to about $16 \mathrm{kPa}(120 \mathrm{~mm} \mathrm{Hg})$ by the end of the pulmonary capillary. However, because the lung is not homogeneously made up of alveolar capillary units that are matched for perfusion and ventilation, the $\mathrm{PaO}_{2}$ in the larger pulmonary veins is lower $(13 \mathrm{kPa}, 100 \mathrm{~mm} \mathrm{Hg})$. This is explained in more detail below. The gradient of carbon dioxide is much more gradual, falling from about $7 \mathrm{kPa}(52 \mathrm{~mm} \mathrm{Hg})$ in the venous system and pulmonary artery to about $5 \mathrm{kPa}(37 \mathrm{~mm} \mathrm{Hg})$ in the pulmonary vein and in the arterial system.

\subsubsection{Arterial oxygen tension V/Q matching}

In the upright position, ventilation and perfusion are both greatest at the bases of the lung. Ventilation is highest at the bases since the lung is most compressed at the base due to its weight and the lower intrapleural pressure, such that on inspiration it expands more. Perfusion is also greatest at the base due to the relatively low perfusion pressure in the pulmonary circulation. In disease, the pulmonary vasculature also maximises $\mathrm{PaO}_{2}$ by ensuring that the well-ventilated areas of the lung receive most of the pulmonary blood flow, a process called V/ $\mathrm{Q}$ matching. This is largely achieved through a process called hypoxic pulmonary vasoconstriction (HPV). ${ }^{52}$ The pulmonary circulation is unique in this regard compared with all the other vascular beds in the body which dilate in response to hypoxia at a threshold of around $8 \mathrm{kPa}(60 \mathrm{~mm} \mathrm{Hg}){ }^{52}$ In poorly ventilated areas of lung, the precapillary pulmonary arterioles constrict in response to sensing low alveolar $\mathrm{PO}_{2}\left(\mathrm{PAO}_{2}\right)$. This is a compensating process and, despite it, some deoxygenated blood may still leave poorly ventilated alveolar capillary units. Deoxygenated blood leaving poorly ventilated alveolar capillary units cannot be compensated for by mixing with blood from well-ventilated units as the relationship between $\mathrm{PaO}_{2}$ and $\mathrm{CaO}_{2}$ is not linear. This physiological phenomenon is

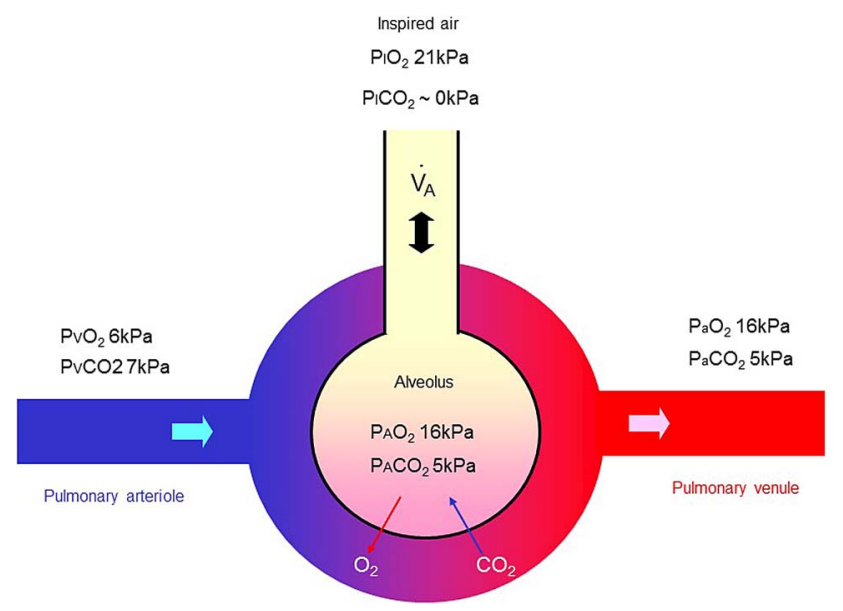

Figure 3 Regulation of $\mathrm{CaO}_{2}$. Alveolar capillary unit. $\mathrm{CaO}_{2}$, blood oxygen content; $\mathrm{PaCO}_{2}$, arterial carbon dioxide and oxygen tensions; $\mathrm{PaO}_{2}$, arterial oxygen tension; $\mathrm{PACO}_{2}$, alveolar carbon dioxide tension; $\mathrm{PAO}_{2}$, alveolar oxygen tension; $\mathrm{PICO}_{2}$, inspired carbon dioxide tension; $\mathrm{PIO}_{2}$, inspired oxygen tension; $\mathrm{PVCO}_{2}$, venous carbon dioxide tension; $\mathrm{PVO}_{2}$, venous oxygen tension. often not fully appreciated and therefore is worth a theoretical worked example (see box).

A much less studied phenomenon that regulates V/Q matching is hypoxic bronchodilation. This effect increases ventilation to poorly ventilated areas of the lung. ${ }^{53}$

\section{Ventilatory drive}

If $\mathrm{PaO}_{2}$ falls, the peripheral chemoreceptors in the carotid body drive an increase in ventilation to increase $\mathrm{PaO}_{2} .{ }^{54}$ This will not significantly increase $\mathrm{PO}_{2}$ in blood leaving already wellventilated units, but will increase $\mathrm{PO}_{2}$ leaving less well-ventilated alveolar units by increasing $\mathrm{PAO}_{2}$ in these units. Although the ventilatory response to $\mathrm{SaO}_{2}$, and therefore $\mathrm{CaO}_{2}$, is linear (figure 4), the carotid body senses $\mathrm{PaO}_{2}$ and not $\mathrm{CaO}_{2}$. This prevents excessive ventilation in response to anaemia which would be ineffective in increasing $\mathrm{CaO}_{2}$. The peripheral chemoreceptors are able to do this because the very high ratio of $\mathrm{DO}_{2}$ to oxygen consumption of the carotid body means that the tissue $\mathrm{PO}_{2}$ in the carotid body continues to reflect $\mathrm{PaO}_{2}$ and will not fall even in the presence of anaemic hypoxia. ${ }^{55} 56$

\section{Example}

- Before oxygen therapy assume $50 \%$ of pulmonary flow is passing through an area of low V/Q and that the pulmonary venous oxyhaemoglobin saturation $\left(\mathrm{SpvO}_{2}\right)$ from this compartment is $80 \%$ (ie, just above mixed venous $\mathrm{SO}_{2}$ ). The other $50 \%$ is passing through an area of matched $V / Q$, resulting in a $\mathrm{SpvO}_{2}$ of $97 \%$. The final mixed $\mathrm{SpvO}_{2}$ will be $88.5 \%$.

- Following maximal oxygen therapy, assuming no change in flow as a result of release of $\mathrm{HPV}, \mathrm{SpvO}_{2}$ from the low V/Q compartment rises to $85 \%$ and $\mathrm{SpvO}_{2}$ from the matched compartment rises to a maximum of $100 \%$. The resulting admixed $\mathrm{SpvO}_{2}$ will now only be $92.5 \%$. This has occurred because fully saturated blood cannot increase its oxygen content beyond full saturation despite an increase in $\mathrm{PO}_{2}$, apart from the minimal contribution from dissolved oxygen; that is, the relationship between $\mathrm{PO}_{2}$ and oxyhaemoglobin saturation/blood oxygen content is not linear.

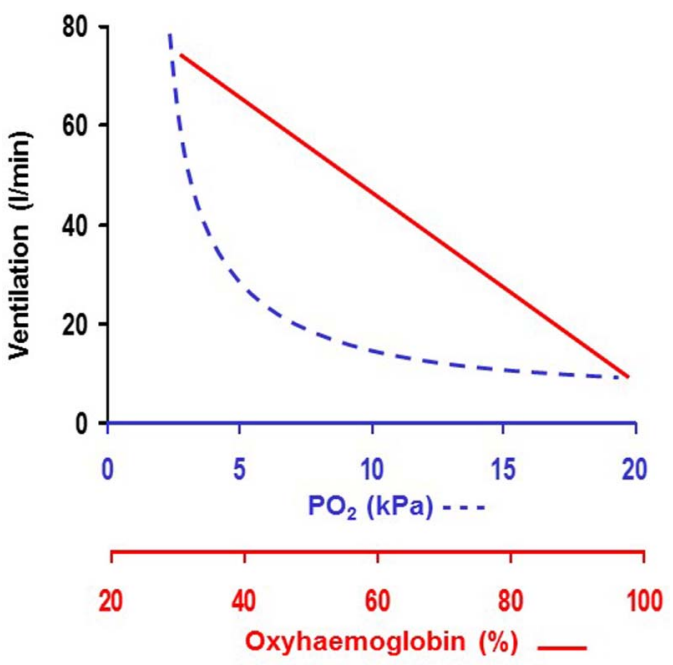

Figure 4 Ventilatory response to hypoxaemia. The relationship is inversely linear when plotted against oxyhaemoglobin (solid line) saturation but inversely exponential when plotted against arterial $\mathrm{PO}_{2}$ (dashed line). $\mathrm{PO}_{2}$, oxygen tension. 


\subsubsection{Haematocrit}

Erythropoiesis is controlled by a negative feedback system involving erythropoietin. By contrast with the carotid bodies, the peritubular cells in the kidney are well suited to sensing oxygen delivery as oxygen extraction is relatively high compared with oxygen delivery. ${ }^{57}$ Although oxygen delivery to the kidneys as a whole organ is high due to high renal blood flow, $\mathrm{DO}_{2}$ is reduced to the renal medulla as oxygen can pass from arterioles to the postcapillary venous system by shunt diffusion due to the parallel organisation of arterial and venous systems. ${ }^{42}$ Consequently, the peritubular cellular $\mathrm{PO}_{2}$ is low. It falls to even lower levels following reductions in $\mathrm{DO}_{2}$ either as a result of hypoxaemia or low haematocrit.

\subsubsection{The oxyhaemoglobin dissociation curve and the Bohr effect}

The oxygen-haemoglobin dissociation curve shows the relationship between haemoglobin $\mathrm{SaO}_{2}$ and $\mathrm{PaO}_{2}$ (figure 5 and table 10). The curvilinear shape of the curve has two particular features that serve to protect from tissue hypoxia. The upper portion of the curve is flat which means that a marked fall in $\mathrm{PaO}_{2}$ is still compatible with a nearly complete oxygen saturation. Second, the steep portion of the curve means that despite rapidly falling oxyhaemoglobin saturation, the $\mathrm{PO}_{2}$ remains relatively well preserved. This property facilitates the continued delivery of oxygen to the tissues despite progressively lower levels of saturation. ${ }^{59}$

The oxygen-carrying capacity of haemoglobin is regulated in response to other metabolic factors to increase the efficiency of oxygen pick-up and delivery. ${ }^{60}$ The curve is shifted to the right because of an increase in temperature, $\mathrm{PaCO}_{2}$ and hydrogen ion concentration (low $\mathrm{pH}$ ) or an increase in 2,3-diphosphoglycerate (DPG). This shift to the right enhances release of oxygen to the tissues and improves oxygen availability, named the Bohr effect (figure 5).

The converse holds true for the lungs where lower carbon dioxide levels favour oxygen loading of haemoglobin.

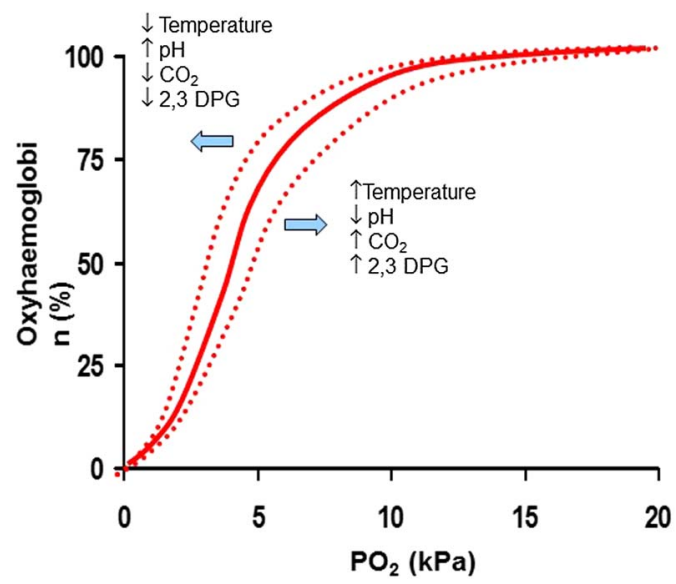

Figure 5 Oxygen dissociation curve with Bohr effect. 2,3-DPG, 2,3-diphosphoglycerate; $\mathrm{PO}_{2}$, oxygen tension.
Consequently, $\mathrm{SaO}_{2}$ cannot accurately predict $\mathrm{PaO}_{2}$ and vice versa, but table 10 gives approximate equivalents.

Chronic hypoxaemia increases 2,3-DPG in erythrocytes, shifting the dissociation curve to the right and therefore increasing oxygen delivery to the tissues. Levels of 2,3-DPG are reduced in stored blood reducing to zero after about 2 weeks. This may reduce oxygen delivery to the tissues but the magnitude of this effect is not thought to be clinically significant. Once transfused, levels of 2,3-DPG increase to about 50\% normal after about 6 hours and back to normal within 48 hours.

\subsubsection{Regulation of $\mathrm{DO}_{2}$}

Acutely, the cardiovascular effects of hypoxaemia will tend to counter the impact of lower $\mathrm{CaO}_{2}$ on $\mathrm{DO}_{2}$ by increasing cardiac output through increased heart rate and myocardial contractility and by decreasing afterload by reducing systemic vascular resistance. $^{61} 62$ Anaemic hypoxia is sensed in the aortic body, presumably owing to lower perfusion relative to oxygen consumption. Consequently, the aortic body can act as a sensor of reduced oxygen delivery as a result of either low $\mathrm{PO}_{2}$ or low haematocrit (unlike the carotid body). ${ }^{55}$

At local tissue level, oxygen delivery can be adjusted to changes in local oxygen consumption. For example, exercising skeletal muscle receives a greater proportion of total cardiac output than resting skeletal muscle. This relates in part to hypoxaemia recruiting a larger proportion of the capillary bed by the relaxation of pericytes, and also through arteriolar vasodilation. $^{63}$

\subsection{Pathophysiology of hypoxia and hyperoxia}

Hypoxia may result from a number of different diseases discussed in section 8 of this guideline.

\subsubsection{Hypoxaemic hypoxia}

Hypoxaemic hypoxia in blood leaving an alveolar capillary unit in the lung may be induced by alveolar hypoxia or incomplete gas exchange (see definition in section 3.2). This may be due to a reduction in inspired oxygen partial pressure, that is, high altitudes, intrapulmonary shunt, V/Q mismatching, alveolar hypoventilation or diffusion impairment.

The alveolar gas equation calculates the oxygen level in the alveolus using the following formula:

$$
\mathrm{PAO}_{2} \approx \mathrm{PIO}_{2}-\mathrm{PACO}_{2} / \mathrm{RER}
$$

where $\mathrm{PAO}_{2}$ and $\mathrm{PACO}_{2}$ represent alveolar levels of oxygen and carbon dioxide, RER is the respiratory exchange ratio or the ratio of carbon dioxide production to oxygen consumption and inspired $\mathrm{PO}_{2} \quad\left(\mathrm{PIO}_{2}\right)=\mathrm{FiO}_{2} \times($ barometric pressure $(100 \mathrm{kPa}$, $750 \mathrm{~mm} \mathrm{Hg}$ - -water vapour pressure $(\sim 6 \mathrm{kPa}, 45 \mathrm{~mm} \mathrm{Hg}))$.

Considering this equation, alveolar hypoxia can be induced by decreased $\mathrm{PIO}_{2}$ or increased $\mathrm{PACO}_{2}$. If an alveolar capillary unit is relatively underventilated for its degree of perfusion (low $\mathrm{V} / \mathrm{Q}$ ratio), $\mathrm{PACO}_{2}$ will rise due to inadequate clearance and thus $\mathrm{PAO}_{2}$ will fall. This may happen for a number of reasons such as increased dead space ventilation during the non-

Table 10 Approximate relationship between $\mathrm{SaO}_{2}$ and $\mathrm{PaO}_{2}$

\begin{tabular}{|c|c|c|c|c|c|c|c|c|c|c|c|c|c|c|}
\hline Oxygen tension $\mathrm{PaO}_{2}(\mathrm{kPa})$ & 4 & 5 & 6 & 7 & 8 & 9 & 10 & 11 & 12 & 13 & 14 & 15 & 16 & $\geq 17$ \\
\hline Oxygen tension $\mathrm{PaO}_{2}(\mathrm{~mm} \mathrm{Hg})$ & 30 & 37.5 & 45 & 52.5 & 60 & 67.5 & 75 & 82.5 & 90 & 97.5 & 104 & 112.5 & 120 & $\geq 127.5$ \\
\hline Oxygen saturation $\mathrm{SaO}_{2}(\%)$ & 57.4 & 71.4 & 80.7 & 86.8 & 90.7 & 93.2 & 94.9 & 96.2 & 97.0 & 97.8 & 98.2 & 98.6 & 98.8 & $\geq 99.0$ \\
\hline
\end{tabular}


fatiguing pattern of shallow respiration in respiratory failure or abnormal lung mechanics in advanced COPD. In diseases that cause global hypoventilation such as respiratory muscle weakness, effectively all areas of lung have low V/Q ratios and this explains the hypercapnia and hypoxaemia associated with these conditions.

An extreme form of low V/Q pathophysiology occurs in intrapulmonary and extrapulmonary shunt where no gas exchange occurs at all. An example of intrapulmonary shunt is when the airway to a lung segment is obstructed by mucus creating an area of lung tissue that is perfused but not ventilated, thus acting as a right-to-left shunt. An example of extrapulmonary shunt is a ventricular septal defect with right-to-left shunting in Eisenmenger's syndrome.

In health and at rest, $\mathrm{PO}_{2}$ has equilibrated with the tissue $\mathrm{PO}_{2}$ across the alveolar capillary membrane one-third of the way along the length of the capillary. With increased thickness of this membrane, as in fibrotic lung disease, equilibration may take longer and an oxygen gradient may persist between the alveolus and blood at the end of the capillary. The overall effect of this when multiple alveolar capillary units are affected will lead to an increased alveolar-to-arterial (A-a) gradient. This is exacerbated during exercise, when capillary transit time decreases.

\subsubsection{Other mechanisms of hypoxia}

Anaemia and carbon monoxide poisoning may result in 'anaemic hypoxia' by reducing oxygen-carrying capacity (see definitions in section 3.2). A low cardiac output state will reduce oxygen delivery even in the absence of hypoxaemia. Tissue hypoxia may develop in these circumstances and this is often termed 'stagnant hypoxia'. 'Histotoxic hypoxia' is due to an inability to metabolise oxygen at the mitochondrial level and may be due to severe sepsis or is seen in certain types of poisoning.

\subsubsection{Hyperoxia}

Hyperoxia can be caused by hyperoxaemia and polycythaemia. Considering again the alveolar gas equation in the previous section, hyperoxaemia can only exist in the presence of high $\mathrm{PIO}_{2}$ or low $\mathrm{PACO}_{2}$ (resulting from hyperventilation). The term 'hyperoxia' could technically be used to describe a patient with polycythaemia without hyperoxaemia, but most clinicians use the term only to describe situations in which the $\mathrm{PaO}_{2}$ is raised.

\subsection{Physiology of carbon dioxide}

\subsubsection{Normal carbon dioxide homoeostasis}

Carbon dioxide is principally carried in the blood in three forms: carbon dioxide, bicarbonate and as a carbamino compound. ${ }^{64}$ In the normal physiological range of 4.6-6.1 $\mathrm{kPa}$ (34$46 \mathrm{~mm} \mathrm{Hg}$ ) the relationship between $\mathrm{PaCO}_{2}$ and carbon dioxide content can be considered linear (figure 6).

\subsubsection{Regulation of carbon dioxide}

$\mathrm{PaCO}_{2}$ is sensed at the peripheral ${ }^{54}$ and central chemoreceptors (in the medulla oblongata) by its effect on intracellular $\mathrm{pH} .{ }^{65}$ Consequently, the regulation of $\mathrm{PaCO}_{2}$ is intimately related to $\mathrm{pH}$ homoeostasis (figure 7).

It is often not appreciated how V/Q matching relates to $\mathrm{PaCO}_{2}$. As discussed in section 5.2.1, alveolar capillary units with a low $\mathrm{V} / \mathrm{Q}$ ratio have increased $\mathrm{PACO}_{2}$. Because of the high solubility and diffusibility of carbon dioxide, there is little A-a gradient for carbon dioxide at the end of the capillary, so blood leaving low V/Q alveolar capillary units has a high $\mathrm{PCO}_{2}$.

As described above, areas of low $\mathrm{V} / \mathrm{Q}$ are usually minimised through HPV. It is also thought that a high $\mathrm{PCO}_{2}$ can cause

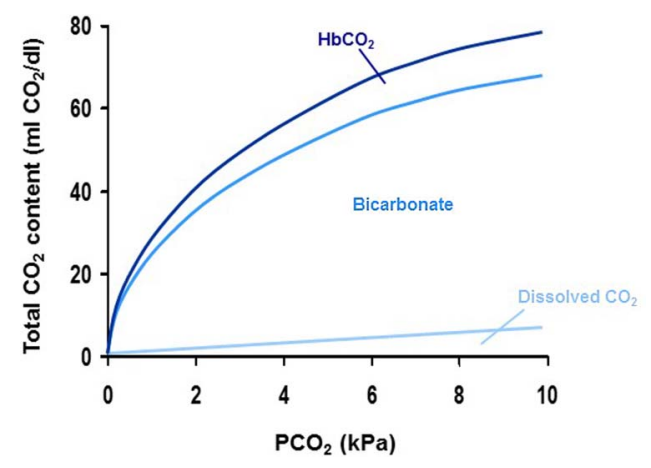

Figure 6 Total carbon dioxide dissociation curve. $\mathrm{HbCO}_{2}$, carbamino haemoglobin; $\mathrm{PCO}_{2}$ carbon dioxide tension.

pulmonary vasoconstriction, adding to the homoeostatic mechanisms of the lung, matching perfusion to ventilation. ${ }^{66} 67$ As the relationship between $\mathrm{PCO}_{2}$ and carbon dioxide dissolved in the blood is approximately linear over the physiological range (unlike oxygen), blood does not become saturated with carbon dioxide and therefore a high pulmonary venous $\mathrm{PCO}_{2}$ from low $\mathrm{V} / \mathrm{Q}$ areas can be partially balanced by a low pulmonary venous $\mathrm{PCO}_{2}$ from high V/Q areas. Consequently, by increasing overall alveolar ventilation, the cardiopulmonary system is able to prevent hypercapnia despite significant V/Q mismatch or shunt, unless respiratory mechanics are limiting.

As with the carriage of oxygen (Bohr effect), there is a reciprocal relationship between $\mathrm{PO}_{2}$ and carbon dioxide carriage. This is known as the Haldane effect. ${ }^{60}$ Deoxygenated haemoglobin has a higher carbon dioxide buffering capacity than oxygenated haemoglobin. This favours carbon dioxide pick-up in the systemic venous circulation and carbon dioxide offloading in the lungs.

Acutely, carbon dioxide acts as a sympathomimetic on the heart: it increases heart rate and stroke volume, increasing cardiac output. Peripherally it causes vasodilation, reducing systemic vascular resistance. Locally, carbon dioxide acts as a vasodilator, thus diverting blood flow to tissues with high metabolic demand. The resulting physical signs of hypercapnia are described in section 7.2.

\subsection{Pathophysiology of hypercapnia and hypocapnia}

\subsubsection{Mechanisms of hypercapnia}

The mechanisms of hypercapnia are simpler than hypoxaemia and there are four possible causes: ${ }^{68}$

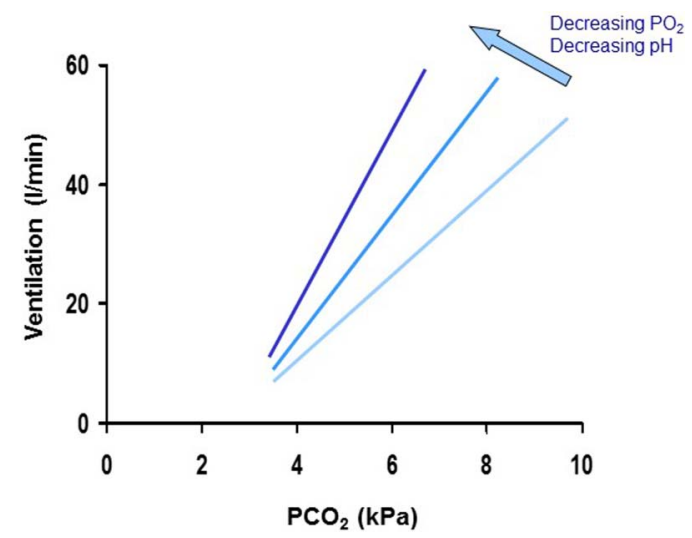

Figure 7 Effect of $\mathrm{PaCO}_{2}$ on ventilation with interaction of acidosis and hypoxaemia. $\mathrm{PaCO}_{2}$, arterial carbon dioxide tension; $\mathrm{PCO}_{2}$ carbon dioxide tension; $\mathrm{PO}_{2}$, oxygen tension. 
1. Increased concentration of carbon dioxide in the inspired gas.

2. Increased carbon dioxide production.

3. Hypoventilation or ineffective ventilation.

4. Increased external dead space.

\section{Mechanisms of hypercapnia}

The mechanisms of hypercapnia in COPD (and other conditions predisposing to hypercapnic respiratory failure) are discussed in section 6.3.1.

\section{Increased concentration of carbon dioxide in the inspired gas}

This iatrogenic cause of hypercapnia is uncommon but should be excluded at the outset in any patient unexpectedly found to be hypercapnic when breathing from, or being ventilated by, external equipment. The severity of hypercapnia due to rebreathing is limited by the rate at which the $\mathrm{PCO}_{2}$ can increase (no more than $0.4-0.8 \mathrm{kPa} / \mathrm{min}, 3-6 \mathrm{~mm} \mathrm{Hg} / \mathrm{min}$ ).

\section{Increased carbon dioxide production}

This is likely only to cause hypercapnia if the minute ventilation is fixed by artificial means and if carbon dioxide production is increased (eg, due to sepsis or increased work of breathing).

\section{Alveolar hypoventilation or ineffective ventilation (deadspace)}

Low alveolar minute ventilation is by far the most common cause of hypercapnia. In clinical practice, COPD is the most common disease to cause hypercapnia; the problem is secondary to alveolar hypoventilation rather than a reduced minute ventilation per se. Patients adopt a rapid shallow pattern of breathing during an AECOPD with the result that the ratio of dead space to tidal volume is increased with more ventilation therefore being 'wasted'. A rapid shallow pattern of breathing results in a bigger proportion of each breath being wasted because of the need to ventilate the anatomical dead space. Furthermore, during acute COPD exacerbations, V/Q mismatch may lead to an increase in physiological dead space, exacerbating the problem further. It is important to note that this commonly occurs in the context of an apparent overall increase in minute ventilation thus hypoventilation being a relative rather than absolute term. Adequate ventilation cannot be achieved since the respiratory muscle 'pump' is unable to overcome the load as a result of underlying respiratory mechanics. Alveolar hypoventilation due to a reduction in minute ventilation is seen following medullary respiratory centre depression by drugs, obstruction of a major airway or restriction of the lungs or chest wall or by respiratory muscle weakness, head injury, intracerebral haemorrhage or opioid narcosis.

Increased external dead space: This would be most common in patients breathing through artificial apparatus which has been incorrectly configured. Although alveolar hypoventilation is the most common cause of hypercapnia, it is important to consider the other potential causes, particularly when patients are receiving assisted ventilation and an artificial breathing circuit is used.

\subsubsection{Hypoventilation and hyperventilation}

Hypoventilation may be physiological-for example, in the face of a metabolic alkalosis. Pathological hypoventilation will occur either when the respiratory muscles are unable to ventilate the lungs sufficiently because they are pathologically weak or they are unable to overcome abnormal lung mechanics such as during an exacerbation of COPD. Reduced respiratory drive caused by drugs with sedative properties or by neurological injury will also produce hypoventilation.
Using the same physiological principles but in reverse, hyperventilation for any reason will produce hypocapnia. This may occur during pure hyperventilation during an anxiety attack or during physiological hyperventilation.

\subsection{Physiology of oxygen therapy}

Oxygen therapy increases $\mathrm{PAO}_{2}$ and is therefore only effective when alveolar capillary units have some functional ventilation. Oxygen therapy is ineffective if there is a pure shunt (such as pulmonary arteriovenous malformations) where mixed venous blood does not pass through an alveolar capillary unit. There will only be a small overall increase in $\mathrm{PaO}_{2}$ due to an increase in dissolved oxygen in the pulmonary venous blood from ventilated alveolar capillary units, which is small compared with the content of oxygen carried by haemoglobin. Despite this, there is good evidence that breath-hold times can be increased by breathing oxygen. ${ }^{69-71}$ One study found that the breath-hold time of 15 healthy participants increased from $56 \mathrm{~s}$ after breathing air to $92 \mathrm{~s}$ after breathing $4 \mathrm{~L}$ of nasal oxygen for $2 \mathrm{~min}$, and another study found that 31 healthy volunteers had an increase in breath-hold time from $32 \mathrm{~s}$ breathing air to $61 \mathrm{~s}$ after breathing oxygen whereas the breath-hold time of 29 patients with chronic pulmonary disease was $9 \mathrm{~s}$ compared with $22 \mathrm{~s}$ for a group of 29 similar patients after breathing oxygen. The same principles are used to preoxygenate patients before intubation during anaesthesia, allowing a longer safe period of apnoea between induction of anaesthesia and passage of an endotracheal tube. It is thought that the additional breath-hold time (and the delay in onset of hypoxaemia following induction of anaesthesia) is produced not by the marginal increase in blood oxygen levels but by the increased reservoir of oxygen in the lungs after breathing oxygen-enriched air.

In poorly ventilated units (ie, low V/Q ratio), $\mathrm{PAO}_{2}$ will be low. Increasing $\mathrm{FiO}_{2}$ will increase $\mathrm{PAO}_{2}$ and therefore $\mathrm{PaO}_{2}$. Hypoventilation disorders can be considered as lungs made up entirely of low V/Q units.

When there is diffusion limitation due to increased alveolar capillary membrane thickness such as in fibrotic lung disease, increasing $\mathrm{PAO}_{2}$ will augment the rate of diffusion across the alveolar capillary membrane by increasing the concentration gradient.

Increasing dissolved oxygen in plasma by oxygen therapy may also be used to offset the effects of hypoperfusion to some extent (stagnant hypoxia) and may well be important in certain situations (cardiogenic shock), although the effect is only marginal. Increased inspired oxygen will only marginally mitigate the effects of anaemic hypoxia but, because the $\mathrm{CaO}_{2}$ in patients with anaemia is less than that in patients with normal haemoglobin, the effect of additional oxygen carried in solution may become more important in these situations.

\subsection{Strategies for improving oxygenation and delivery}

Tissue oxygenation is dependent on optimal or adequate oxygen delivery to the tissue $\left(\mathrm{DO}_{2}\right)$. This physiological process is composed of various components that independently and interdependently influence and determine $\mathrm{DO}_{2}$ and therefore tissue oxygenation. These components can be considered sequentially.

\subsubsection{Optimising $\mathrm{PaO}_{2}$}

The physiology of oxygen therapy has already been discussed in the previous section. However, increasing $\mathrm{FiO}_{2}$ is only one component in increasing oxygen uptake in the lungs. Other key manoeuvres to ensure oxygen delivery to the alveolar capillary bed include: 
- Maintaining a satisfactory airway.

- Ensuring adequate alveolar ventilation.

- Reversing any respiratory depressants such as narcotics.

- Invasive ventilation or NIV where necessary.

- Treating airflow obstruction by bronchodilation or sputum clearance techniques.

- Optimising transfer factor (diffusion capacity).

- Treatment of pulmonary oedema.

\subsubsection{Optimising oxygen carriage}

Oxygen is carried in blood mainly by haemoglobin with only a very small amount of oxygen dissolved in the blood itself. Adequate haemoglobin is therefore essential for $\mathrm{CaO}_{2}$. The ideal haemoglobin level for optimal $\mathrm{CaO}_{2}$ and therefore for optimal $\mathrm{DO}_{2}$ has long been a subject of debate. Previous practices have favoured haemoglobin levels close to $100 \mathrm{~g} / \mathrm{L}(10 \mathrm{~g} /$ $\mathrm{dL}$ ), providing adequate $\mathrm{CaO}_{2}$ as well as reducing viscosity of blood for better perfusion in critically ill patients. However, studies by Canadian researchers in the late 1990s have shown that haemoglobin levels of $70 \mathrm{~g} / \mathrm{L}(7 \mathrm{~g} / \mathrm{dL})$ were as safe as higher levels and may produce fewer complications in the critically ill. ${ }^{72}$ However, this study was conducted using non-leucocyte depleted blood and it is possible that some of the infective complications in the group who were given more transfusions might have been avoided by the use of leucocyte-depleted blood. The optimal transfusion target for critically ill patients therefore remains the subject of ongoing discussion among experts in critical care medicine. Although the issue of optimal haemoglobin in patients with unstable or symptomatic coronary artery disease is not settled, haemoglobin levels of $100 \mathrm{~g} / \mathrm{L}(10 \mathrm{~g} / \mathrm{dL})$ are recommended for adequate $\mathrm{DO}_{2}$ (see box).

\subsubsection{Optimising delivery}

Besides adequate $\mathrm{CaO}_{2}$ and $\mathrm{PaO}_{2}$, delivery of oxygen depends on adequate flow of oxygenated blood. Cardiac output in turn depends on adequate blood (circulating) volume, adequate venous return and adequate and optimal myocardial function. To avoid tissue hypoxia, attention must therefore be paid to the volume status of the patient and the adequacy of cardiac function, as well as initiating oxygen therapy. In severely shocked patients (eg, cardiogenic shock, septic shock), invasive monitoring and inotropic/vasopressor therapy will usually be indicated in appropriate higher dependency environments. Some studies have shown that deliberately increasing oxygen delivery in critically ill patients as well as high-risk surgical patients reduces organ failure, reduces length of ICU stay and, most importantly, improves mortality. ${ }^{73-76}$ However, there is a substantial body of evidence showing no favourable effect on morbidity and mortality by achieving supranormal values for the cardiac index or normal values for mixed venous oxygen saturation. ${ }^{77-79}$

\section{SECTION 6: HYPOXIA, HYPOXAEMIA, HYPEROXAEMIA, HYPERCAPNIA AND THE RATIONALE OF TARGETED OXYGEN THERAPY}

\subsection{Effects and risks of hypoxia/hypoxaemia and rationale for target oxygen saturation range}

As this guideline is addressing emergency oxygen therapy and oxygen therapy in healthcare settings, this section will focus on the effects and risks of acute hypoxaemia. Section 8 will discuss the emergency treatment of acute hypoxaemia in patients with long-term diseases associated with chronic hypoxaemia. The approximate relationship between $\mathrm{PaO}_{2}$ and $\mathrm{SaO}_{2}$ at normal $\mathrm{pH}$ and temperature is shown in table 10 and figure 5 (oxygen dissociation curve). The effects and risks of hypoxaemia are summarised in table 11 . Severe hypoxaemia may lead to brain damage and death. In general, many of the physiological effects of hypoxaemia are mediated by low $\mathrm{PaO}_{2}$, irrespective of oxygen content. For example, even when the total blood oxygen content is normal in the presence of polycythaemia, hypoxaemia will still exert a physiological effect such as stimulation of ventilation. The risks of hypoxaemia, however, are usually mediated by low tissue $\mathrm{PO}_{2}$ which may occur as a consequence of a low $\mathrm{PaO}_{2}$ and other mechanisms such as severe anaemia and low cardiac output states. These problems can be illustrated in the pathophysiology of myocardial ischaemia which will develop when there is an imbalance between myocardial $\mathrm{DO}_{2}$ and oxygen consumption $\left(\mathrm{V}^{\cdot} \mathrm{O}_{2}\right) \cdot \mathrm{DO}_{2}$ is not only dependent on $\mathrm{PaO}_{2}$, but also coronary flow and haematocrit. $\mathrm{V}^{\cdot} \mathrm{O}_{2}$ will also depend on the stroke work of the heart. Defining a lower limit of

Table 11 Physiological effects of acute hypoxaemia and hyperoxaemia

\begin{tabular}{|c|c|c|c|c|}
\hline & \multicolumn{2}{|l|}{ Hypoxaemia } & \multicolumn{2}{|l|}{ Hyperoxaemia } \\
\hline & Effects & Risks & Effects & Risks \\
\hline Respiratory system & $\begin{array}{l}\text { Increased ventilation } \\
\text { Pulmonary vasoconstriction }\end{array}$ & Pulmonary hypertension & $\begin{array}{l}\text { Decreased ventilation } \\
\text { (minimal) }\end{array}$ & $\begin{array}{l}\text { Worsened ventilation/perfusion } \\
\text { matching } \\
\text { Absorption atelectasis }\end{array}$ \\
\hline $\begin{array}{l}\text { Cardiovascular } \\
\text { system }\end{array}$ & $\begin{array}{l}\text { Coronary vasodilation } \\
\text { Decreased systemic vascular } \\
\text { resistance (transient) } \\
\text { Increased cardiac output } \\
\text { Tachycardia }\end{array}$ & $\begin{array}{l}\text { Myocardial } \\
\text { Ischaemia/infarction } \\
\text { Ischaemia/infarction of other critically } \\
\text { perfused organs Hypotension } \\
\text { Arrhythmias }\end{array}$ & & $\begin{array}{l}\text { Myocardial ischaemia (in context of } \\
\text { decreased haematocrit) } \\
\text { Reduced cardiac output } \\
\text { Reduced coronary blood flow } \\
\text { Increased blood pressure } \\
\text { Increased peripheral resistance }\end{array}$ \\
\hline Metabolic system & $\begin{array}{l}\text { Increased 2,3-DPG } \\
\text { Increased } \mathrm{CO}_{2} \text { carriage (Haldane } \\
\text { effect) }\end{array}$ & Lactic acidosis & $\begin{array}{l}\text { Decreased 2,3-DPG } \\
\text { Decreased } \mathrm{CO}_{2} \text { carriage } \\
\text { (Haldane effect) }\end{array}$ & Increased reactive oxygen species \\
\hline $\begin{array}{l}\text { Neurological } \\
\text { system }\end{array}$ & $\begin{array}{l}\text { Increased cerebral blood flow due } \\
\text { to vasodilation }\end{array}$ & $\begin{array}{l}\text { Confusion } \\
\text { Delirium } \\
\text { Coma }\end{array}$ & $\begin{array}{l}\text { Decreased cerebral blood } \\
\text { flow }\end{array}$ & \\
\hline Renal system & $\begin{array}{l}\text { Renin-angiotensin axis activation } \\
\text { Increased erythropoietin } \\
\text { production }\end{array}$ & Acute tubular necrosis & & Reduced renal blood flow \\
\hline
\end{tabular}


$\mathrm{PaO}_{2}$ which is considered safe is therefore impossible given the other variables. Hypoxaemia refers to an abnormally low $\mathrm{PO}_{2}$ in the blood (see section 3.2). However, it is not possible to define a single level of hypoxaemia that is dangerous to all patients. Some patients with chronic lung disease may be accustomed to living with $\mathrm{SaO}_{2}$ as low as $80 \%\left(\mathrm{PaO}_{2}\right.$ about $6 \mathrm{kPa}$ or $45 \mathrm{~mm} \mathrm{Hg}$ ) while other patients may be harmed by modest hypoxaemia. It has been shown that medical patients with sustained desaturation $<90 \%$ have impaired mediumterm survival compared with medical patients with saturations which stay $>90 \% .^{29}$

Recent observational data have shown a step-wise increase in mortality in hypoxaemic acute medical patients breathing air; the lowest mortality (3.7\%) was found in patients with saturation $\geq 96 \%$ and this increased incrementally to mortality above $25 \%$ among patients with saturation $<88 \%{ }^{19}$ However, much of this survival disadvantage may be due to the underlying disease which has caused the low oxygen level (eg, severe COPD or pneumonia) with the degree of hypoxaemia being a marker of disease severity. Furthermore, the mean and median oxygen saturation of patients aged $>65$ and the lower limit of the interquartile range (IQR) was about $2 \%$ lower than the saturation level of young adults so it is likely that much of the increase in mortality associated with modest hypoxaemia was related to the age of the patient. Therefore, the contribution of modest hypoxaemia to mortality rates is not known.

Mental functioning becomes impaired if the $\mathrm{PaO}_{2}$ falls rapidly to $<6 \mathrm{kPa}\left(45 \mathrm{~mm} \mathrm{Hg}, \mathrm{SaO}_{2}<80 \%\right)$ and consciousness is lost at $<4 \mathrm{kPa}\left(30 \mathrm{~mm} \mathrm{Hg}, \mathrm{SaO}_{2}<56 \%\right)$ in normal participants. ${ }^{80-83}$ Young participants tolerate acute hypoxaemia for longer than older participants in terms of 'time of useful consciousness ${ }^{83}$ Safe levels of hypoxaemia in COPD have been discussed in detail in a review by Murphy et al. ${ }^{84}$ Many patients with COPD have a $\mathrm{PaO}_{2}$ of $<5 \mathrm{kPa}(37.5 \mathrm{~mm} \mathrm{Hg})$ corresponding to $\mathrm{a} \mathrm{SaO}_{2}$ of $<70 \%$ during an acute exacerbation. ${ }^{85}$ Furthermore, sudden hypoxaemia is more dangerous than hypoxaemia of gradual onset both in health and in disease. For example, millions of people live at altitudes above $3000 \mathrm{~m}$ despite an average $\mathrm{PaO}_{2}$ of about $7.3 \mathrm{kPa}(55 \mathrm{~mm} \mathrm{Hg}$, saturation about $88 \%$ ) and acclimatised climbers on Mount Everest can tolerate short-term exposure to an oxygen saturation of $54 \%$ or less with an estimated $\mathrm{PaO}_{2}$ of about $3.3 \mathrm{kPa}(25 \mathrm{~mm} \mathrm{Hg}) .{ }^{26-}$ $283486 \mathrm{Campbell}^{87}$ summarised this issue eloquently in 1967 when he said "Better a year at a $\mathrm{PaO}_{2}$ of $50 \mathrm{~mm} \mathrm{Hg}(6.7 \mathrm{kPa})$ than an hour at a $\mathrm{PaO}_{2}$ of $20 \mathrm{~mm} \mathrm{Hg}(2.7 \mathrm{kPa}) . "$

Urine flow and renal function have been shown to decrease abruptly when the $\mathrm{PaO}_{2}$ falls below $40 \mathrm{~mm} \mathrm{Hg}(5.3 \mathrm{kPa})$ which corresponds to oxygen saturation of $\sim 74 \%,{ }^{88}$ Hypoxic hepatitis has been reported in patients with respiratory failure associated with oxygen levels below $4.5 \mathrm{kPa}$ or $34 \mathrm{~mm} \mathrm{Hg}$, whereas hypoxic hepatitis in patients with cardiac disease is mainly due to decreased hepatic blood flow (stagnant hypoxia) and occurs at higher blood oxygen levels. ${ }^{89} 90$ An in-flight study of patients with COPD with mean $\mathrm{SaO}_{2}$ of $96 \%$ at sea level showed a fall to $90 \%$ in-flight in a commercial airliner and a further fall to a mean $\mathrm{SaO}_{2}$ of $87 \%$ while walking in the aircraft aisles. These patients had no symptoms during these hypoxaemic episodes. ${ }^{91}$ A study of 84 healthy airline passengers found that the mean $\mathrm{SaO}_{2}$ fell from $97 \%$ at ground level to $93 \%$ (1SD 85-98\%) at cruising altitude. ${ }^{92} \mathrm{~A}$ study of healthy airline cabin crew has shown that the $\mathrm{SaO}_{2}$ of flight attendants falls to a nadir ranging from $80 \%$ to $93 \%$ (mean $88.6 \%$ ) without causing breathlessness or any other symptoms. ${ }^{93}$
Without any randomised evidence, the guideline production team have suggested that the level of saturation which is tolerated by healthy people without any symptoms (about $85 \%$ saturation) should be regarded as the safe lower limit of hypoxaemia. However, other comorbidities may need to be taken into account and expert opinion recommends that the $\mathrm{SaO}_{2}$ should be maintained above $90 \%$ for seriously ill patients. ${ }^{29-32}$ For this reason, the present guideline recommends a target $\mathrm{SaO}_{2}$ (and $\mathrm{SpO}_{2}$ ) $\geq 94 \%$ for most hypoxaemic patients to ensure that the actual oxygen level remains above $90 \%$ for most of the time with a $4 \%$ margin of safety to allow for variability in oxygen saturation levels, their recordings and oximeter error. The accuracy of and pitfalls of oximetry are addressed in section 7.1.2.

Specific targets for oxygen therapy in various diseases will be considered theoretically in this section and practically in sections 8 and 9. Some patients-especially older people or those with chronic lung disease-may have an $\mathrm{SaO}_{2}$ below 94\% when clinically stable and oxygen should not be given just to maintain the $\mathrm{SaO}_{2}$ above $94 \%$ if the patient is clinically stable. In assessing an ill patient, the $\mathrm{SaO}_{2}$ level is only one of several physiological variables that should be monitored. Many patients with sudden acute illness such as postoperative pulmonary emboli will have a sudden alteration in physiological variables as assessed by 'track and trigger' systems such as the modified Early Warning Scoring (mEWS) systems ${ }^{94} 95$ or the UK NEWS. ${ }^{96}$ Such patients may have only a small fall in $\mathrm{SaO}_{2}$ owing to physiological compensation mechanisms such as increased ventilation. Healthcare professionals therefore need to be alert for falls in $\mathrm{SaO}_{2}$ even within the recommended target ranges. Because of the wide normal range for $\mathrm{SaO}_{2}$ and the uncertainty concerning the possible physiological consequences of minor hypoxaemia, there was more debate among the 2008 guideline group about desirable target ranges than about any other aspect of the guideline. If the $\mathrm{SaO}_{2}$ should fall slightly below $94 \%$, the key issue is to identify and treat the cause of the fall (eg, pulmonary embolism) rather than just correcting the hypoxaemia which is not of itself likely to be dangerous at this level. However, there is a danger that healthcare workers might fail to respond appropriately to abnormal hypoxaemia. After much debate it was concluded that the guideline would recommend a target range of $94-98 \%$ for all adult patients. This reflects the approximate normal range of $\mathrm{SaO}_{2}$ in healthy adults as discussed in section 3.1. However, a sustained fall in $\mathrm{SaO}_{2}$ of $>3 \%$, even within the normal range, may be an indicator of acute illness and should require clinical assessment of a patient while a minority of patients (especially those aged $>70$ years) may have an $\mathrm{SaO}_{2}$ of $<94 \%$ even when clinically stable.

\subsubsection{Desirable oxygen saturation ranges in acute illness}

- Acute hypoxaemia is considered dangerous to healthy participants below a $\mathrm{PaO}_{2}$ of about $6 \mathrm{kPa}(45 \mathrm{~mm} \mathrm{Hg})$ or an $\mathrm{SaO}_{2}$ of about $80 \%$ due to impaired mentation and risk of tissue hypoxia, but patients with acute illness or chronic organ disease or ischaemia are likely to be at risk above this level and critical care guidelines recommend aiming to achieve saturation $>90 \%$.

- Changes in physiological 'track and trigger' systems such as the NEWS ${ }^{96}$ may occur in acute illness with either no change or only a small change in $\mathrm{SaO}_{2}$ levels.

- Critical illness may present initially with only a small fall in $\mathrm{SaO}_{2}$ level because of compensating mechanisms. 
- The upper end of the recommended range in this guideline $(98 \%)$ is the upper limit of $\mathrm{SaO}_{2}$ in healthy adults.

- The lower end of the suggested target saturation range (94\%) is close to the lower end of the normal range and ensures that the $\mathrm{SaO}_{2}$ remains above $90 \%$ even if it falls slightly below the target range.

\subsection{Potential benefits of hyperoxaemia and supplemental oxygen therapy in non-hypoxaemic patients}

Supplemental oxygen therapy is most commonly given to correct hypoxaemia as discussed throughout this guideline. However, there are some circumstances where supplemental oxygen may be given to non-hypoxaemic patients to achieve hyperoxaemia. This needs to be distinguished from hyperbaric oxygen therapy (oxygen therapy delivered above atmospheric pressure) which is outside the scope of this guideline.

\subsubsection{Hyperoxaemia has been shown to be beneficial in the} following clinical situations

- Carbon monoxide and cyanide poisoning (see section 8.10.7)

- Spontaneous pneumothorax (see section 8.11.6)

- Some postoperative complications (see section 8.15.3)

- Cluster headache (see section 8.13.7)

\subsubsection{Other potential benefits and potential harms of oxygen therapy in non-hypoxaemic patients}

- Most guidelines for CPR and the care of patients with critical illness recommend the use of the highest feasible oxygen concentration in the initial stages of resuscitation. Although these recommendations are not evidence-based, it is unlikely that controlled trials would ever be undertaken using different levels of oxygen therapy in these emergencies and it seems intuitive to maximise oxygen delivery for critically ill patients with circulatory collapse and respiratory failure. However, randomised trials have been undertaken of resuscitation of neonates breathing room air or oxygen and the unexpected outcome of a Cochrane review was that the survival was better when room air was used. ${ }^{97}$ This surprising finding cannot be extrapolated to adult patients, but it does emphasise the need for clinical trials even in areas where one might intuitively believe that oxygen would be beneficial. Furthermore, there is theoretical evidence that patients who have had a restoration of spontaneous circulation after cardiac arrest may be managed more safely with $<100 \%$ inspired oxygen. ${ }^{98} 99$ Recent observational studies suggesting possible harmful outcomes from hyperoxaemia in survivors of cardiopulmonary resuscitation are discussed in section 8.10.1.

- It has been shown that early intervention to increase oxygen delivery to the tissues in critically ill patients as well as highrisk surgical patients reduces organ failure, reduces length of ICU stay and, most importantly, improves survival. ${ }^{73-76}$ Increased oxygen delivery in part involves oxygen therapy, but these studies did not show any benefit from aiming at supraphysiological oxygen delivery. There is some evidence that hyperoxaemia may be associated with increased mortality in patients on ICUs, this is discussed in section 8.10.2.

- Reported benefits of oxygen therapy in healing of established wounds and in treatment of wound sepsis are controversial. Hyperbaric oxygen reduced the risk of amputation in patients with chronic diabetic foot ulcers and may improve the chance of healing over 1 year, but the Cochrane reviewers had concerns about the size and quality of existing studies and recommended further trials. ${ }^{100}$ It is not known if conventional oxygen therapy has any effect on wound healing. ${ }^{101}$

- The potential benefit of oxygen for prevention of postoperative wound infection is discussed in section 8.15.3.

- Through relief of breathlessness and work of breathing, oxygen therapy may decrease carbon dioxide production and consequently offset some of the potential increase in $\mathrm{PaO}_{2}$ that might otherwise occur due to the mechanisms described in section 6.3.1. However, there are no controlled trials supporting the use of oxygen for this indication.

\subsection{Potential adverse physiological effects and clinical risks of supplemental oxygen therapy and hyperoxaemia}

These are summarised in table 11 and in the review by Downs $^{102}$ and in other sources. ${ }^{103-107}$ The following paragraphs will summarise the physiology and pathophysiology of supplemental oxygen therapy and hyperoxaemia.

\subsubsection{Respiratory system}

The most significant effect of high-concentration oxygen therapy on the respiratory system is hypercapnic respiratory failure in a population of vulnerable patients as described more fully in section 8.12. This does not occur in the absence of significant pulmonary disease or musculoskeletal disease affecting the thorax, and it can occur while the $\mathrm{PaO}_{2}$ is still within the normal range or slightly below normal, especially in patients with exacerbated COPD for whom the baseline oxygen saturation level may be well below the lower limit of normal.

It has been stated for several decades that hyperoxaemia causes hypercapnic respiratory failure by producing decreased respiratory drive in patients with intrinsic lung disease, such as COPD', and many-if not most-medical textbooks from the 1960 s to the present time refer to 'loss of hypoxic drive' as the main cause of hypercapnia and acidosis when highconcentration oxygen is given to patients with an AECOPD. This assertion is usually attributed to Campbell who championed the concept of controlled oxygen therapy in the $1960 \mathrm{~s}$. However, Campbell ${ }^{87}$ has been widely misquoted. What he actually said in 1967 was as follows: "It is usual to attribute the rise in $\mathrm{PaCO}_{2}$ in these patients to removal of the hypoxic drive to ventilation but I share the doubts of Pain and co-workers ${ }^{108}$ that this is the whole story; changes in the pulmonary circulation may also be important." Most but not all subsequent studies $^{48} 49$ 108-113 have shown that Campbell was correct in this assumption as discussed in section 4.2. Another curious feature of hypercapnia in AECOPD is that it is not universal. ${ }^{87}$ Some patients with COPD are prone to repeated episodes of hypercapnic respiratory failure and others may not ever suffer from this complication. Even among patients with COPD with chronic hypercapnia, not all will develop an increased carbon dioxide level (and acidosis) during acute exacerbations. The theory of 'loss of hypoxic drive' as the cause of hypercapnia is further confounded by the observation that $\mathrm{PaCO}_{2}$ continues to rise as $\mathrm{PaO}_{2}$ is increased above $13 \mathrm{kPa}(100 \mathrm{~mm} \mathrm{Hg})$, which has little impact on decreasing ventilation and most patients with respiratory acidosis during an exacerbation of COPD have $\mathrm{PaO}_{2}$ above $10 \mathrm{kPa}$, equivalent to saturation above about $93 \%{ }^{43}$ Therefore, while a small reduction in ventilation may be a contributing factor to the rise in carbon dioxide levels during oxygen therapy in COPD, the major factor is the worsening of $\mathrm{V} / \mathrm{Q}$ matching. Additional effects of increasing $\mathrm{FiO}_{2}$ will relate to atelectasis and perhaps worsening airflow obstruction due to increased viscosity. In conditions where there is little intrinsic lung disease but significant respiratory muscle weakness, loss of 
hypoxic respiratory drive will be a greater factor in the development of hypercapnia. However, HPV remains a significant regulator of $\mathrm{V} / \mathrm{Q}$ matching even in non-diseased lung.

While the mechanism of oxygen-induced hypercapnia remains controversial it is likely there are at least six mechanisms responsible for this. These are ranked in likely order of importance below:

- V/Q mismatch,

- Ventilatory drive,

- Haldane effect,

- Absorption atelectasis,

- Higher density of oxygen compared with air,

- Rebreathing can occur if low oxygen flow rates are used through face masks.

\section{V/Q mismatch}

During air breathing, poorly ventilated alveolar capillary units will be hypoxic and therefore poorly perfused due to HPV. If high-concentration oxygen is administered, the $\mathrm{PAO}_{2}$ will rise, thus reversing the HPV and increasing blood flow to that unit. However, although the oxygen in the unit has increased, it remains poorly ventilated with a high $\mathrm{PACO}_{2}$ and therefore a high pulmonary venous $\mathrm{PCO}_{2}$. As more blood is now passing through these units, the $\mathrm{PaCO}_{2}$ will rise. Normally when there is no significant lung disease or thoracic musculoskeletal disease, the respiratory system is able to compensate for these changes by increasing overall ventilation thereby lowering $\mathrm{PaCO}_{2}$. However, where respiratory mechanics are such that increased ventilation is limited, $\mathrm{PaCO}_{2}$ will rise. Several authors have reported that this mechanism is more important than reduction in ventilatory drive in producing hypercapnia when supplementary oxygen is administered, but this continues to be a controversial area of respiratory physiology. ${ }^{48} 49$ 108-113

\section{Ventilatory drive}

Hypoxaemia drives an increase in ventilation so it follows that relief of hypoxaemia will cause a decrease in ventilation. The consequent rise in $\mathrm{PaCO}_{2}$ is inversely proportional to the decrease in ventilation such that a halving of alveolar ventilation will lead to a doubling in $\mathrm{PaCO}_{2}$, assuming constant carbon dioxide production. As shown in figure 4, any increase in $\mathrm{PaO}_{2}$ above $8 \mathrm{kPa}(60 \mathrm{~mm} \mathrm{Hg})$ will not significantly reduce ventilation and increases above $13 \mathrm{kPa}(100 \mathrm{~mm} \mathrm{Hg})$ will have little impact on ventilation as the carotid sinus discharge is largely attenuated above $13 \mathrm{kPa}$. This mechanism is therefore only of importance in explaining increases in $\mathrm{PaCO}_{2}$ when $\mathrm{PaO}_{2}$ rises to $13 \mathrm{kPa}$, but not increases thereafter. This phenomenon is also seen in normal individuals. Several clinical studies have suggested that 'hypoxic drive' makes only a small contribution to the rise in $\mathrm{PaCO}_{2}$ that is seen clinically when high-concentration oxygen is given to patients with COPD, but one recent study has supported this mechanism. ${ }^{48} 49108-113$

\section{Haldane effect}

The third effect of increasing $\mathrm{FiO}_{2}$ is to decrease the carbon dioxide buffering capacity of haemoglobin through the Haldane effect $^{60}$ (see section 5.3.2).

\section{Absorption atelectasis}

The fourth effect, absorption atelectasis, is thought to occur as a result of absorption of oxygen from alveoli with high $\mathrm{PAO}_{2}$ beyond obstructed airways. This can happen at $\mathrm{FiO}_{2}$ as low as $30-50 \%$ and will result in a shunt (increased V/Q mismatch). ${ }^{102}$

\section{Higher density of oxygen compared with air}

Johnson et al ${ }^{114}$ have shown a reduction in $\mathrm{FEV}_{1}$ in patients who were breathing pure oxygen compared with breathing air. They concluded that this effect was probably related to the slightly increased density and viscosity of oxygen relative to air. This would increase the work of breathing which could contribute to hypercapnia in an exhausted patient.

\section{Rebreathing from face masks with low-flow rates}

This issue is discussed in section 10 and in a paper by Jensen et al. ${ }^{115}$

\subsubsection{Rebound hypoxaemia following sudden cessation of supplementary oxygen therapy}

Patients who have developed decompensated hypercapnic respiratory failure following high-concentration oxygen therapy face a further significant danger of rebound hypoxaemia if oxygen is suddenly withdrawn in an attempt to correct the effects of excess oxygen therapy. ${ }^{116} 117$

Rebound hypoxaemia can be explained using the alveolar gas equation and, given its importance, is best illustrated with a working example (box). For the purposes of simplicity, this example makes several assumptions such as a constant respiratory exchange ratio and $\mathrm{A}-\mathrm{a}$ gradient between stages 1 and 3. It also assumes that ventilation remains unchanged. Although when $\mathrm{PaO}_{2}$ falls to $3.4 \mathrm{kPa}$ on removal of oxygen, ventilation will rise, by definition it will not be able to rise sufficiently to meet the need to clear the carbon dioxide stores for the same reason that hypercapnic respiratory failure developed in the first instance. Rebound hypoxaemia is a major risk and could cause death. Consequently, this guideline will recommend that oxygen therapy be stepped down gradually while monitoring saturation continuously.

Good practice point

$\checkmark$ Sudden cessation of supplementary oxygen therapy can cause rebound hypoxaemia with a rapid fall in $\mathrm{PO}_{2}$ to below the tension that was present prior to the start of supplementary oxygen therapy.

\section{Example: rebound hypoxaemia}

Stage 1: consider a patient with an exacerbation of COPD whose $\mathrm{ABGs}$ are as follows: $\mathrm{PaO}_{2} 6.5 \mathrm{kPa} ; \mathrm{PaCO}_{2} 7.5 \mathrm{KPa}$ breathing room air. The $\mathrm{PAO}_{2}$ calculated from the alveolar gas equation (5.2.1) will be 11.6 assuming a respiratory exchange ratio of 0.8 , giving an $\mathrm{A}-\mathrm{a}$ gradient of $5.1 \mathrm{kPa}$.

Stage 2: given maximal oxygen through a reservoir bag mask, his blood gases become: $\mathrm{PaO}_{2} 32 \mathrm{kPa} ; \mathrm{PaCO}_{2} 10 \mathrm{kPa}$. Because of the high solubility of carbon dioxide, the total body stores of carbon dioxide will have risen.

Stage 3: If oxygen therapy is suddenly withdrawn, $\mathrm{PACO}_{2}$ and $\mathrm{PaCO}_{2}$ will remain high initially because of the high carbon dioxide stores and therefore $\mathrm{PAO}_{2}$ will fall further than it was initially before oxygen therapy to $8.5 \mathrm{kPa}$. Assuming the A-a gradient for oxygen stays at $5.1 \mathrm{kPa}$ for room air breathing, then calculated $\mathrm{PaO}_{2}$ will become $3.4 \mathrm{kPa}$.

\subsubsection{Cardiovascular and cerebrovascular system}

The effects and potential harms of hyperoxaemia on the circulation have been summarised by Thomson et al ${ }^{105}$ in an editorial which made a strong case for more trials. Hyperoxaemia causes coronary and cerebral vasoconstriction and, if the haematocrit is sufficiently low, this may theoretically cause paradoxical tissue hypoxia because of overall reduction in $\mathrm{DO}_{2}$. There have been reports of possible harm from the administration of oxygen to non-hypoxaemic patients with strokes of mild-to-moderate 
severity or to non-hypoxaemic patients with myocardial infarction as discussed in sections 8.13.1 and 8.13.2. Thomson et $a l^{105}$ have suggested that oxygen should be 'prescribed, administered and monitored with care' in order 'to achieve optimal tissue oxygenation', not maximal oxygenation. This view was proposed by other authors such as Bryan and Jenkinson ${ }^{118}$ in the 1980 s, but standard medical practice had not taken note of this advice prior to the publication of the first version of this guideline in 2008. Because there are no published data suggesting benefit from hyperoxaemia for most medical conditions and because of the theoretical risks, optimal management should aim for physiological oxygenation. Targets for oxygen therapy in specific circumstances, with evidence, are discussed in section 8 .

\subsubsection{Reactive oxygen species, tissue toxicity and reports of increased mortality}

Aside from the potentially detrimental physiological effects of hyperoxaemia, the toxic effects mediated by reactive oxygen species (ROS) have potential risk. ${ }^{118}$ Excess ROS are generated in the presence of high tissue $\mathrm{PO}_{2}$ in the form of hydrogen peroxide and superoxide, causing oxidative stress and free radical damage. ${ }^{119}$ At physiological levels ROS act as signalling molecules, but at higher levels they are cytotoxic, notably being released by primed neutrophils as a host defence mechanism. It is thought that ROS are responsible for the development of bronchopulmonary dysplasia in ventilated hyperoxygenated premature infants $^{120}$ and reperfusion injury postmyocardial infarction. ${ }^{121}$ It has been known since the 19th century that prolonged exposure to high concentrations of oxygen in animal models leads to diffuse alveolar damage, haemorrhage, alveolar collapse, infiltration of inflammatory cells, necrosis, apoptosis and injury to the endothelium and epithelium in the lungs which causes death in the rat model after 4 days of exposure to $73 \%$ oxygen at one atmosphere. $^{122}$ Griffith et $a l^{123}$ demonstrated in 1986 that normal human lungs are injured by breathing 30-50\% oxygen for 45 hours with leakage of albumin into bronchoalveolar lavage fluid and other markers of lung injury. ${ }^{123}$

\subsubsection{Delay in recognition of physiological deterioration}

It was previously believed that a high $\mathrm{FiO}_{2}$ is protective and gives patients a margin of safety. However, Downs ${ }^{102}$ and Beasley et $a l^{106}$ have argued that unstable patients may actually be placed at risk by the precautionary use of high-concentration oxygen therapy. ${ }^{107} 124$ During physiological deterioration, a patient given high-concentration oxygen therapy would have a normal or high pulse oximeter reading masking a progressive decline in the $\mathrm{PaO}_{2} / \mathrm{FiO}_{2}$ ratio and therefore not alerting staff to impending deterioration requiring mechanical support. A worked example of this is shown in reference. ${ }^{106}$ Furthermore, a patient who deteriorated physiologically while at a low $\mathrm{FiO}_{2}$ would be detected early by pulse oximetry and could have the $\mathrm{FiO}_{2}$ increased while being transferred to an ICU, whereas a patient who was already receiving a high $\mathrm{FiO}_{2}$ would desaturate more slowly but, when the oximeter eventually detected desaturation, there would be fewer treatment options because increasing the $\mathrm{FiO}_{2}$ further would have little effect. ${ }^{88} 92107$

\subsubsection{Acute lung injury in patients with acute paraquat poisoning,} bleomycin lung injury and acid aspiration

Oxygen is known to be hazardous to patients with paraquat poisoning ${ }^{125} 126$ and oxygen potentiates bleomycin lung injury and may potentiate lung injury from aspiration of acids. ${ }^{127-129}$
Further details concerning these conditions are given in section 8.13.4.
6.3.7 Summary of risks of hyperoxaemia and supplemental oxygen therapy
Physiological risks
1. Worsened V/Q mismatch.
2. Absorption atelectasis.
3. Coronary and cerebral vasoconstriction.
4. Reduced cardiac output.
5. Damage from oxygen free radicals.
6. Increased systemic vascular resistance.

\section{Clinical risks}

1. Worsening of hypercapnic respiratory failure.

2. Delay in recognition of clinical deterioration.

3. Potentially worse outcomes in mild-to-moderate stroke.

4. Specific risk in patients with previous bleomycin lung damage or with paraquat poisoning or acid aspiration.

5. Unknown risk-benefit balance in acute coronary artery disease with normal oxygen saturation.

6. Association with increased risk of death in survivors of cardiac arrest and among patients on ICUs.

7. Uncontrolled supplemental oxygen therapy can be harmful to patients who are at risk of hypercapnic respiratory failure, especially if the $\mathrm{PaO}_{2}$ is raised above $10 \mathrm{kPa}$.

8. High-concentration oxygen therapy to produce hyperoxaemia (above normal oxygen saturation) and has been associated with increased risk of death in some patient groups (eg, patients with mild and moderate strokes, survivors of cardiac arrests and ICU patients; see section 8).

\subsection{Risks of hypercapnia (and respiratory acidosis)}

Hypercapnia and respiratory acidosis are inextricably linked and are best considered together. If hypercapnia develops slowly (over several days), a patient will have renal compensation (retention of bicarbonate) and acidosis will not occur in most such cases. However, acute elevation of the blood carbon dioxide level produces respiratory acidosis and symptoms of hypercapnia. Some of the consequences of an elevated $\mathrm{PCO}_{2}$ are a consequence of the resulting acidosis. Sometimes the effect of a raised $\mathrm{PCO}_{2}$ on a particular organ system is opposed by an opposite effect of acidosis. Carbon dioxide is a vasodilator and patients with hypercapnia may appear flushed with dilated peripheral veins and a bounding pulse. Cranial vasodilation may cause headache. Carbon dioxide in high concentrations has hypnotic effects and patients with hypercapnia may progress from drowsiness to confusion to coma. ${ }^{84}$ 130-134 A link has been shown between inappropriately high blood oxygen levels due to highconcentration oxygen use and the development of severe respiratory acidosis in acute COPD with increased risk of death or requirement for mechanical ventilation. ${ }^{43} \quad 46 \quad 135$ However, the problem of respiratory acidosis is not confined to patients with COPD. Depressed respiration for any reason will give rise to hypercapnia. Examples are opioid overdoses, obesity with hypoventilation and neuromuscular disorders affecting the muscles of respiration. Recent studies have shown a rise in the carbon dioxide level in patients with OHS and also in patients with pneumonia and with acute exacerbations of asthma where the link between hyperoxaemia and hypercapnia was not previously recognised. ${ }^{47} 136137$ 
An audit of blood gases from 4866 patients at a university hospital has shown that hypercapnia was commoner than hypoxaemia in blood gas samples from surgical wards, a surgical HDU, theatre and the ICU as well as on medical wards and the medical HDU and many of these hypercapnic patients had respiratory acidosis. ${ }^{42}$ Furthermore, a Swiss audit of 530 blood gas specimens in the emergency department showed that patients in the lowest $\mathrm{pH}$ tertile more often required admission to ICU $(28 \%$ vs $12 \%$ in the first tertile, $\mathrm{p}<0.001)$ and had higher in-hospital mortality $(14 \%$ vs $5 \%, \mathrm{p}=0.003){ }^{138}$ Hypercapnia on admission was independently associated with increased risk of ICU admission and increased 30-day mortality in that study.

\subsubsection{Effects of a raised blood carbon dioxide level Nervous system}

Carbon dioxide exerts its effect either directly or as a consequence of acidosis. Hypercapnia increases cerebral blood flow and thereby may influence the cerebrospinal fluid pressure. It is the main factor influencing the intracellular $\mathrm{pH}$ which has an important effect on cellular metabolism. It exerts an inert gas narcotic effect similar to that of nitrous oxide. It influences the excitability of neurones particularly relevant in the reticular activating system. Carbon dioxide can induce narcosis when the $\mathrm{PaCO}_{2}$ rises above $12-16 \mathrm{kPa}(90-120 \mathrm{~mm} \mathrm{Hg}){ }^{130}$

Pulmonary circulation

An elevated $\mathrm{PACO}_{2}$ causes vasoconstriction in the pulmonary circulation although the effect is less marked than that of hypoxaemia. ${ }^{139}$ In healthy participants an end expiratory $\mathrm{PCO}_{2}$ of $7 \mathrm{kPa}(52 \mathrm{~mm} \mathrm{Hg})$ increases pulmonary vascular resistance by $32 \%$ which, along with raised cardiac output, increases mean pulmonary artery pressure by $60 \% .{ }^{140}$ Changes in $\mathrm{pH}$ are thought to be the primary factor responsible for carbon dioxidemediated changes in the pulmonary vasculature. ${ }^{141} 142$ Consequently, as with HPV, changes in $\mathrm{PACO}_{2}$ help to match perfusion to ventilation.

Respiratory system

As explained in section 5.2.1, a raised carbon dioxide level may worsen hypoxaemia and its effects because the concentration of carbon dioxide in the alveolar gas reduces that of oxygen if the concentration of nitrogen remains constant. Also an increase in $\mathrm{PaCO}_{2}$ shifts the oxygen dissociation curve to the right.

Cardiovascular system

In general, both hypercapnia and acidosis have direct depressant effects on cardiac myocytes and vascular smooth muscle cells. ${ }^{143}$ These effects are normally opposed by the increase in catecholamines caused by the raised $\mathrm{PaCO}_{2}$. The overall effect of carbon dioxide on the cardiovascular system is therefore unpredictable. In artificially ventilated children a rise in carbon dioxide increases cardiac output and reduces total peripheral resistance and blood pressure tends to rise. ${ }^{144}$ Although an increase in carbon dioxide depresses heart rate, tachycardia is more common because of the effects of catecholamine stimulation over-riding the depressant effects on the heart. Arrhythmias have been reported but are seldom clinically significant in normal participants. Carbon dioxide is a systemic vasodilator.

\section{Kidneys}

Renal blood flow and glomerular filtration rate are reduced abruptly in the presence of levels of $\mathrm{PaCO}_{2}$ above about $65 \mathrm{~mm} \mathrm{Hg}(8.7 \mathrm{kPa}) .{ }^{88} 145$

Blood electrolyte levels

The acidosis that accompanies hypercapnia may cause a rise in potassium if the acidosis is severe and sustained.

\section{Endocrine system}

Hypercapnia increases plasma levels of endogenous adrenaline and noradrenaline.

\subsubsection{Clinical signs}

The clinical signs of hypercapnia are produced by the physiological changes described above and are described in detail in section 7.2.1.

\subsection{Risks of acidosis}

The major effect of acidosis is depression of the central nervous system with severe acidosis $\left(\mathrm{pH}, 7.0\right.$ or $\left.\left[\mathrm{H}^{+}\right] 100 \mathrm{nmol} / \mathrm{L}\right)$ causing disorientation and later coma. However, as described above, the effects of $\mathrm{pH}$ are inextricably linked with both hypoxaemia and hypercapnia. As a consequence of opposing effects of acidosis, hypoxaemia and hypercapnia on different target organs in individual patients together with the fact that derangements of all three components may occur at the same time, it is very difficult to predict the effects of acidosis per se in an individual patient. Furthermore, tissue hypoxia will exacerbate acidosis. The consequences will depend on the interplay of the three variables, complicated by the effects of comorbid disease states. It is well known that, in patients with COPD, a pH of $<7.30$ or $\left[\mathrm{H}^{+}\right]>50 \mathrm{nmol} / \mathrm{L}$ during an acute exacerbation is associated with a much worse prognosis. ${ }^{43}$

\subsection{Rationale of oxygen therapy}

Oxygen therapy is usually defined as the administration of oxygen at concentrations greater than those found in ambient air. It is usually undertaken to treat or prevent hypoxaemia, thereby preventing tissue hypoxia which may result in tissue injury or even cell death. Clinicians must bear in mind that supplemental oxygen is given to improve oxygenation but it does not treat the underlying causes of hypoxaemia which must be diagnosed and treated as a matter of urgency.

In some circumstances such as carbon monoxide poisoning or cyanide poisoning or cluster headache, oxygen therapy is used to achieve hyperoxaemia. There is evidence from randomised studies that oxygen does not relieve breathlessness compared with air in non-hypoxaemic patients with COPD who are breathless following exertion or in non-hypoxaemic patients with advanced cancer. ${ }^{146-150}$ At the tissue level, mitochondrial activity requires oxygen for aerobic ATP synthesis for cellular activity. $\mathrm{PaO}_{2}$ of dry air at sea level is $21.2 \mathrm{kPa}(159 \mathrm{~mm} \mathrm{Hg})$, but at the mitochondrion, $\mathrm{PO}_{2}$ is in the range of $0.5-3.0 \mathrm{kPa}$ (4-22 $\mathrm{mm} \mathrm{Hg}$ ) depending on tissue type and local metabolic activity. This gradient from atmosphere to mitochondrion is known as the oxygen cascade. There are many factors in this cascade that affect the final mitochondrial $\mathrm{PO}_{2}$ including alveolar gas exchange, oxygen transport in the blood and tissue perfusion. Under pathological conditions, any change in one step in this cascade may result in hypoxia at the mitochondrial level. Therefore, although not necessarily addressing the underlying cause of tissue hypoxia, increasing $\mathrm{FiO}_{2}$ with oxygen therapy is the simplest and quickest way of avoiding hypoxic tissue damage in patients with hypoxaemia. Besides oxygen therapy, other steps are usually necessary to improve the delivery of oxygen to the tissue (see section 5.6).

\subsection{Target oxygen saturation in acute illness}

Many disease states lead to hypoxaemia and it is standard practice for breathless patients to be treated with oxygen (see also section 4.3). However, there have been few controlled trials comparing different levels of inspired oxygen for patients with 
any of the common diseases that lead to hypoxaemia. It must also be remembered that oxygen therapy is only one of several strategies that may be used to increase tissue oxygen delivery for critically ill patients (see section 5.6). In many clinical situations oxygen therapy is applied without a specific end point in mind. It has been suggested by many studies that hyperoxaemia can have deleterious physiological and clinical effects (see section 6.3), albeit such effects are not widely reported in conditions other than COPD. However, potential for harm may well exist with hyperoxaemia and good medical practice should be followed as in all drug prescriptions. This is especially important in view of recent suggestions by some authors that hyperoxaemia may be associated with increased risk in ICU patients and in survivors of cardiac arrest although this view has been challenged by other authors. ${ }^{151-156}$ As the actual $\mathrm{PO}_{2}$ at the mitochondrial level is so variable and dependent on many variables other than $\mathrm{PaO}_{2}$, it is often difficult to set a minimum level of $\mathrm{PaO}_{2}$ below which definite cell damage will occur or above which the host is safe from the effects of hypoxic cell damage. In addition, it is not possible to monitor mitochondrial $\mathrm{PO}_{2}$ clinically and the only clinically available surrogate of mitochondrial hypoxia is lactate production. Although blood lactate levels are useful and may indicate tissue hypoxaemia, it is a late marker and therefore is an insensitive tool. Thus, targets set for 'ideal' blood gas levels are based on arbitrary goals.

Owing to the natural decline in normal arterial oxygen levels with age, it has been suggested that the ideal target $\mathrm{PaO}_{2}$ can be determined by the following equation: ideal $\mathrm{PaO}_{2}=13.3 \mathrm{kPa}$ $-0.04 \times$ age (in years) or $100 \mathrm{~mm} \mathrm{Hg}-0.3 \times$ age (in years). ${ }^{157} \mathrm{In}$ terms of oxygen saturation measured by the bedside, this would translate into an $\mathrm{SaO}_{2}$ of $94-98 \%$ in most situations. This strategy avoids tissue hypoxia in almost all patients and also avoids potential deleterious effects of hyperoxaemia. Thus, the standard practice should be to prescribe oxygen to a specific saturation (or $\mathrm{PaO}_{2}$ ) rather than in terms of $\mathrm{FiO}_{2}$. Clearly, consideration will need to be given to patients who have oxygen-sensitive carbon dioxide retention and targets may well have to be set lower for these patients (usually 88-92\%) to strike a balance between achieving a desirable and safe $\mathrm{SaO}_{2}$ / $\mathrm{PaO}_{2}$ and carbon dioxide retention. There has been one randomised trial of controlled oxygen therapy with target range 88$92 \%$ in patients with exacerbated COPD. ${ }^{51}$ This trial is supported by five decades of physiological and observational studies which are reviewed in section 8.12. Specific disease states will be addressed in section 8. Patients with moderate-to-severe hypoxaemia are usually breathless and have an increased respiratory rate. Apart from causing physical tiredness, this also increases work of breathing, therefore increasing both oxygen consumption and carbon dioxide production. In these circumstances, oxygen therapy may reduce the work of breathing and therefore reduce carbon dioxide production. Therefore, oxygen therapy should theoretically improve breathlessness in hypoxaemic patients. However, despite the widespread belief of doctors and patients that oxygen relieves breathlessness, little evidence of benefit effect has been found in clinical trials involving patients who were breathless but not hypoxaemic. For example, one meta-analysis of all published blinded studies of short burst oxygen therapy for patients with COPD with breathlessness reported modest and heterogeneous benefit and another meta-analysis failed to confirm any clinical benefit. $^{146} 158$

A 2004 systematic review of oxygen and airflow on the relief of breathlessness at rest in patients with advanced disease of any cause found only low-grade scientific evidence that oxygen and airflow improve breathlessness in some patients with advanced disease at rest, and almost all of these participants were hypoxaemic and already using oxygen therapy. ${ }^{159}$ A study published in 2010 reported no additional symptomatic benefit of oxygen over air delivered by nasal cannulae for relieving refractory breathlessness related to life-limiting illness in patients with $\mathrm{PaO}_{2}>55 \mathrm{~mm} \mathrm{Hg}(7.3 \mathrm{kPa})$. Breathlessness decreased in both study arms, temporally related to provision of medical gas. ${ }^{150}$

Evidence statements

- There have been no randomised trials comparing different oxygen saturation levels for patients not at risk of hypercapnic respiratory failure. Expert opinion (based on physiology and observational studies) recommends maintaining normal or near-normal oxygen saturation of $94-98 \%$ for these patients (evidence level 4).

- The target saturation in patients with COPD who are at risk of hypercapnia is $88-92 \%$ (evidence level $1+$ ).

- The target saturation for patients with other risk factors for hypercapnia (eg, morbid obesity, chest wall deformities or neuromuscular disorders) is $88-92 \%$ based on expert opinion which is extrapolated from observational studies (evidence level 4).

Recommendations for achieving desirable oxygen saturation ranges in acute illness (see also charts 1-2)

A1: This guideline recommends aiming to achieve a normal or near-normal oxygen saturation for all acutely ill patients apart from those at risk of hypercapnic respiratory failure (grade D).

A2: The recommended target saturation range for acutely ill patients not at risk of hypercapnic respiratory failure is 9498\% (grade D).

A3: For most patients with known COPD or other known risk factors for hypercapnic respiratory failure (eg, morbid obesity, CF, chest wall deformities or neuromuscular disorders or fixed airflow obstruction associated with bronchiectasis), a target saturation range of $88-92 \%$ is suggested pending the availability of blood gas results (grade A for COPD, grade D for other conditions).

A4: Most non-hypoxaemic breathless patients do not benefit from oxygen therapy, but a sudden reduction of $\geq 3 \%$ in a patient's oxygen saturation within the target saturation range should prompt fuller assessment of the patient (and the oximeter signal) because this may be the first evidence of an acute illness (grade D).

\subsection{Effects of body positioning including restraint systems}

Appropriate positioning of a patient can maximise V/Q matching. In the healthy self-ventilating adult lung, $\mathrm{V} / \mathrm{Q}$ matching improves from non-dependent to dependent areas. In lung disease, there is a disruption of this pattern and, in these instances, appropriate positioning may be advantageous in optimising V/Q matching, therefore improving gas exchange, oxygenation and carbon dioxide clearance. For these reasons, breathless patients usually prefer to sit upright or near upright provided they are able to do so. The relationship between dependency and V/Q matching is maintained irrespective of the position of the participant. The physiology is then transferable into alternate side lying positions; for example, in left side lying the dependent lung (left) will have the better V/Q matching. This is important in the presence of asymmetrical lung pathology as the 'good lung down' principle will maximise V/Q matching. Many unwell patients are nursed in the semirecumbent and supine positions. These positions do not facilitate V/Q matching as in the upright and full side lying position due to 
the hindrance to expansion of the dependent lung by the diaphragm and chest wall. Even in healthy participants the $\mathrm{PO}_{2}$ is $0.7 \mathrm{kPa}(5 \mathrm{~mm} \mathrm{Hg})$ lower in the supine position than in the upright position. ${ }^{20}$ Similarly, $10 \%$ of patients with right hemiparesis and concomitant chest disease were more hypoxaemic in the left lateral position. ${ }^{160}$ Where there is pathological lung disease and hence already significant $\mathrm{V} / \mathrm{Q}$ mismatch, gas exchange may be further impaired. This is discussed in a review of the effects of position on oxygen saturation in acute stroke. ${ }^{161}$ Patients with acute stroke without respiratory comorbidities may be permitted to adopt any body position that they find most comfortable, while those with respiratory compromise should be positioned as upright as possible, avoiding slouched or supine positions to optimise oxygenation. ${ }^{161}$ The semirecumbent/supine position is commonly adopted in an ambulance. In addition, for safety, the patient is strapped into the stretcher using abdominal and chest restraints with their arms by their side. While there are a lack of specific data regarding this, physiological principles suggest that the use of such positioning and restraints would compromise both respiratory muscle function and gas exchange. Finally, there are some rare patients with liver disease, cardiac shunts or lung fibrosis who have 'platypnoea and orthodeoxia' which means that they are more hypoxic in the upright position. ${ }^{162}$ Other patients with scoliosis or with a paralysed hemidiaphragm may feel more comfortable with the 'good lung up'. These patients should be allowed to choose the position in which their breathing is most comfortable for them.

\section{Evidence statement}

- Evidence from a number of cohort studies shows that oxygenation is reduced in the supine position, both in healthy participants and in acute illness but there are no controlled trials showing benefit from specific body positions (evidence level 4) Recommendation

A5: Because oxygenation is reduced in the supine position, fully conscious hypoxaemic patients should ideally be allowed to maintain the most upright posture possible (or the most comfortable posture for the patient) unless there are good reasons to immobilise the patient (eg, skeletal or spinal trauma) (grade D).

\section{SECTION 7: CLINICAL AND LABORATORY ASSESSMENT OF HYPOXAEMIA AND HYPERCAPNIA}

\subsection{Assessment of hypoxaemia}

7.1.1 Clinical assessment of breathless patients and assessment of cyanosis

Clinicians examining a critically ill patient should remember the 'ABC' of emergency medicine. In the case of critically ill patients it may be necessary to secure the airway and resuscitate a patient before a detailed history can be obtained and before a full physical examination can be undertaken. Additionally, it is important to remember that supplemental oxygen is given to improve oxygenation but it does not influence the underlying cause(s) of hypoxaemia which must be diagnosed and treated as a matter of urgency.

In assessing an ill patient the $\mathrm{SpO}_{2}$ level is only one of several physiological variables that should be monitored. Many patients with sudden acute illness such as postoperative pulmonary emboli will have a sudden alteration in physiological 'track and trigger' variables as assessed by the mEWS system ${ }^{94} 95163$ and the NEWS developed by the Royal College of Physicians. ${ }^{96}$ Such patients may have only a small fall in $\mathrm{SpO}_{2}$ due to physiological compensation mechanisms such as increased ventilation. Clinicians therefore need to be alert for falls in $\mathrm{SpO}_{2}$ even within the recommended target ranges.
Although the use of 'track and trigger' systems are strongly recommended, the guideline group had some reservations of their use in patients at risk of hypercapnic respiratory failure, and in particular, the 2012 version Royal College of Physicians NEWS as there was no option to set a target range for oxygen saturations. ${ }^{164} 165$ Patients at risk of hypercapnic respiratory failure who are within the target range of $88-92 \%$ as recommended in this guideline would score 2-3 EWS points, and may prompt nursing staff to increase the inspired oxygen level to achieve 'normal' oxygenation $(>96 \%)$ and put the patient at risk. The guideline group recommends use of the 2017 update of the NEWS chart which allows appropriate adjustments to the scoring for saturation for patients at risk of hypercapnic respiratory failure. Patients should score EWS points for saturations below the target range or if significantly above the target range while breathing oxygen. The 2017 NEWS chart will have a special oximetry section for patients at risk of hypercapnia.

\section{Evidence statement}

- There are no controlled trials of different methods of assessment of acutely unwell patients. Advice on clinical assessment is based on expert opinion and retrospective studies of outcomes (evidence level 4).

\section{Recommendations}

B1: Fully trained clinicians should assess all acutely ill patients by measuring respiratory rate, pulse rate, blood pressure and temperature, and assessing circulating blood volume and anaemia. Expert assistance from specialists in intensive care or from other disciplines should be sought at an early stage if patients are thought to have major life-threatening illnesses and clinicians should be prepared to call for assistance when necessary including a call for a 999 ambulance in prehospital care or a call for the resuscitation team or ICU outreach team in hospital care (grade D).

Traditional clinical assessment of hypoxaemia involves clinical inspection of the skin and buccal mucous membranes to decide whether central cyanosis is present or absent. This is a difficult clinical skill, especially in poor lighting conditions. Clinical assessment of hypoxaemia is made even more unreliable by the presence of anaemia or polycythaemia. Some patients may have peripheral cyanosis due to poor peripheral circulation in the presence of normal $\mathrm{SaO}_{2}$. Several studies have shown that hypoxaemia is often not recognised by emergency medical service providers, especially if the patient does not complain of respiratory distress. ${ }^{166-168}$ A systematic review of the literature in 2005 reported that most hypoxaemic patients had at least one vital sign abnormality but skin colour was a poor indicator of hypoxaemia compared with pulse oximetry. ${ }^{39}$ For these reasons it is recommended that clinicians should not rely on visual assessments of 'cyanosis' but should instead use pulse oximetry to obtain an accurate assessment of a patient's oxygen saturation.

The nature of a patient's presenting illness may make hypoxaemia a likely outcome, thus prompting a careful clinical search for evidence of cyanosis complemented by urgent pulse oximetry. This situation applies to many common acute illnesses such as heart failure, COPD exacerbation, pneumonia and pulmonary embolism. A study of 2276 patients with pneumonia showed that hypoxaemia was independently associated with six risk factors: age $>30$ years (OR 3.2), COPD (OR 1.9), congestive heart failure (OR 1.5), respiratory rate $>24 / \mathrm{min}$ (OR 2.3), altered mental status (OR 1.6) and chest radiographic infiltrate involving $>1$ lobe (OR 2.2). ${ }^{36}$ Acutely ill patients with significant hypoxaemia are likely to have an increased pulse rate or respiratory rate and, for this reason, usually score several NEWS points. ${ }^{94} 95163165169$ 
The respiratory rate is the single best predictor of severe illness. ${ }^{95}$ However, many patients with marked hypoxaemia may present with non-specific findings such as restlessness and confusion rather than breathlessness, and oxygen saturation has been shown to be an independent predictor of mortality in multivariate analysis of the outcome of emergency medical admissions. ${ }^{19}{ }^{170}$ Furthermore, the work of Thrush et $a l^{171}$ on normal volunteers has shown that heart rate, blood pressure and respiratory rate are not reliable indicators of hypoxaemia down to saturation levels as low as $70 \%$. This would suggest that the changes in vital signs which are seen in most hypoxic patients are due to the underlying illness rather than hypoxaemia per se.

Hypoxaemia may be associated with increased or decreased ventilation. Although some hypoxaemic patients may have reduced levels of ventilation as a causative factor, the majority of hypoxaemic patients have increased minute ventilation in an attempt to increase the blood oxygen level. For example, a patient with an opioid overdose may have reduced ventilation causing hypoxaemia despite having structurally normal lungs, whereas a patient with pneumonia or major pulmonary embolism may have significant hypoxaemia due to $\mathrm{V} / \mathrm{Q}$ mismatch despite an increased level of ventilation. The first patient in this example may appear peaceful and non-distressed despite significant hypoventilation and hypoxaemia, while the second patient is likely to have increased ventilation and tachycardia. The clinician therefore needs to make separate assessments of a patient's oxygen saturation and level of ventilation.

Having completed the history and rapid assessment of the patient, more detailed physical examination may reveal signs of an illness such as major pleural effusion, major pneumothorax or unexpected heart failure that may prompt the clinician to anticipate the presence of hypoxaemia.

Good practice points for clinical assessment of patients with suspected hypoxaemia

$\checkmark$ The medical history should be taken when possible in an acutely breathless patient and may point to the diagnosis of a particular acute illness such as pneumonia or pulmonary embolism or an exacerbation of a chronic condition such as COPD, asthma or heart failure.

$\checkmark$ Never discontinue oxygen therapy to obtain an oximetry measurement on room air in patients who clearly require oxygen therapy.

$\checkmark$ Physical examination should be undertaken urgently. This may provide evidence of a specific diagnosis such as heart failure or a large pleural effusion, but it is common for the cause of breathlessness to remain undiagnosed until the results of tests such as chest radiographs are available.

$\checkmark$ Record arterial oxygen saturation measured by pulse oximetry $\left(\mathrm{SpO}_{2}\right)$ and consider blood gas assessment in patients with unexplained confusion and agitation as this may be presenting feature of hypoxaemia and/or hypercapnia (cyanosis is a difficult physical sign to record confidently, especially in poor light or with an anaemic or plethoric patient).

$\checkmark$ Carefully measure respiratory rate and heart rate because tachypnoea and tachycardia are more common than a physical finding of cyanosis in hypoxaemic patients.

$\checkmark$ Appropriate changes should be made to any 'track and trigger' system used to allow for a lower target range in patients at risk of hypercapnic respiratory failure. These patients should score no EWS points for saturation if within their target range and they should score points if the oxygen saturation falls below the target range or if the saturation rises above the target range while breathing oxygen. The 2017 update of the NEWS chart has a special section for oximetry measurements for use with patients who have target range $88-92 \%$ and it is recommended that the 2017 NEWS chart should be used in all hospitals (see recommendation B4).

$\checkmark$ The presence of a normal $\mathrm{SpO}_{2}$ does not negate the need for blood gas measurements especially if the patient is on supplemental oxygen therapy. Pulse oximetry will be normal in a patient with a normal oxygen tension $\left(\mathrm{PO}_{2}\right)$ but abnormal carbon dioxide tension $\left(\mathrm{PCO}_{2}\right)$ or with a low blood oxygen content due to anaemia. For this reason, blood gases and full blood count tests are required as early as possible in all situations where these measurements may affect patient outcomes.

$\checkmark$ All clinical staff who use oximeters must be trained in their use and made aware of the limitations of oximetry. (Oximetry is a valuable clinical tool but subject to artefact and errors of interpretation).

\subsubsection{Value and limitations of pulse oximetry}

Clinical assessment of hypoxaemia has been revolutionised by the advent of pulse oximetry in much the same manner as the clinical assessment of blood pressure was transformed by the invention of the sphygmomanometer. However, it was common in the past to see patients with acute respiratory illness who have had multiple measurements of their blood pressure but no record made of their oxygen saturation, peak expiratory flow or $\mathrm{FEV}_{1}$. In addition to the clinical consequences of underassessment, Howes et $a l^{172}$ and Macnab et $a l^{173}$ have reported that the availability of a pulse oximeter was highly cost-effective because the finding of normal oximetry (>94\%) in many patients allowed paramedics to use oxygen less frequently with a potential financial saving of up to $\$ 2324(\sim £ 1200)$ per ambulance per annum. The availability now of highly portable, low-cost pulse oximeters should make the measurement of oxygen saturations in many clinical scenarios commonplace and used as the 'fifth vital sign'. We would encourage all clinical staff who are involved in the care of acutely ill patients to carry and use one so that measurement of oxygen saturations is as normal a part of assessment of a patient as listening to their chest.

Pulse oximetry measures haemoglobin oxygen saturation by detecting the absorption of light at two specific wavelengths that correspond to the absorption peaks of oxygenated and deoxygenated haemoglobin. Oximeters are less reliable at low saturation such as $80 \%$, but modern oximeters reflect the $\mathrm{SaO}_{2}$ accurately at saturation above about $88 \% .^{174-178}$ In almost all clinical circumstances covered by this guideline, patients with a saturation below $88 \%$ will be given intensive therapy to bring the saturation up to at least $90 \%$, so the inaccuracy of the instruments at very low saturation levels should not affect patient management.

In one study of 123 adult patients who had simultaneous measurements of pulse oximetry and $\mathrm{SaO}_{2}$ measured in $\mathrm{ABG}$, the $95 \%$ CI for the median difference ranged from -0.6 to $+0.5 \% .{ }^{176}$ It has been estimated that an oxygen saturation of $92 \%$ or above measured by pulse oximetry has a sensitivity of $100 \%$ and specificity of $86 \%$ for excluding hypoxaemia defined as an arterial oxygen partial pressure below $60 \mathrm{~mm} \mathrm{Hg}(8 \mathrm{kPa}) .{ }^{179}$

Oximetry may be less accurate in acutely ill patients on ICUs, but there are no direct comparisons of the accuracy of pulse oximetry in critically ill patients compared with stable patients and healthy individuals. The study of Perkins et $a l^{180}$ showed a mean $\mathrm{SpO}_{2}$ of $94.6 \%$ compared with a mean $\mathrm{SaO}_{2}$ of $95.9 \%$ from 1132 simultaneous oximeter and ABG measurements on an ICU. Fortunately, this average difference of $1.3 \%$ was lower for pulse oximeter readings, thus allowing a margin of safety in most cases. This study also showed that fluctuations in oxygen 
saturation measured by oximetry tended to be greater than changes in $\mathrm{SaO}_{2}$ measured with samples from an indwelling radial artery catheter.

Although oximetry is widely used, there are few clinical studies examining its utility. The Cochrane meta-analysis of the use of oximetry in perioperative monitoring of more than 20000 patients failed to show any reduction in complications or deaths where oximetry was used, although oxygen was given more often to patients who were monitored with pulse oximetry. ${ }^{181}$ The authors suggested that the correction of modest hypoxaemia probably does not have much effect on clinical outcomes.

Pulse oximetry gives no information concerning $\mathrm{pH}, \mathrm{PCO}_{2}$ or haemoglobin level. Blood gases and full blood count tests are therefore required as early as possible in all situations where these measurements may affect patient outcomes.

The accuracy of pulse oximetry is diminished in patients with poor peripheral perfusion which may occur chronically in conditions such as systemic sclerosis or acutely in patients with hypotension or hypovolaemia. However, it has been suggested that many types of oximeter may remain accurate at arterial pressures as low as $20 \mathrm{~mm} \mathrm{Hg}$ so long as the machine is able to obtain a reading despite the low pulse pressure. ${ }^{182}$ Most oximeters give an indication of the pulse signal strength. It is important to ensure that the oximeter has a good signal if technically possible, and the probe may need to be tried on different fingers or toes or on the earlobe to obtain the best available signal for the individual patient. There are some patients with poor perfusion for whom pulse oximetry measurements cannot be made. This includes patients with cold peripheries (eg, Raynaud's phenomenon), severe hypotension and peripheral 'shut down'.

It must be remembered that oximetry gives a normal reading for oxygen saturation in most patients with anaemia because the oxygen saturation of the available haemoglobin is normal although the total amount of haemoglobin available for oxygen transport is reduced. These patients have normal oxygen saturation levels despite having 'anaemic hypoxia' which may cause considerable reduction in the total oxygen content of the blood. It is often not recognised that a patient with an $\mathrm{SpO}_{2}$ of $98 \%$ but a haemoglobin of $7 \mathrm{~g} / \mathrm{dL}\left((7 \times 0.98 \times 1.34)=9.2 \mathrm{~mL} \mathrm{O}_{2} / \mathrm{dL}\right)$ will have a greatly reduced blood oxygen content compared with a patient with a haemoglobin of $15 \mathrm{~g} / \mathrm{dL}$ and a saturation of $85 \%((15 \times 0.85 \times 1.34)=17 \mathrm{~mL} \mathrm{O} / \mathrm{dL}$; each g/dL haemoglobin when fully saturated carries $1.34 \mathrm{~mL}$ oxygen).

The accuracy of oximetry is unreliable in the presence of carbon monoxide or methaemoglobin. Both of these substances have similar light absorption characteristics to oxyhaemoglobin so an apparently normal $\mathrm{SpO}_{2}$ in a patient with carbon monoxide poisoning or methaemoglobinaemia may be falsely reassuring. Carboxyhaemoglobin levels above $2 \%$ may cause falsely elevated $\mathrm{SpO}_{2}$ measurements. ${ }^{183}$ Many smokers will have carboxyhaemoglobin levels above this level shortly after smoking a cigarette, and the carboxyhaemoglobin level may be elevated to $15 \%$ in some smokers and up to $50 \%$ or more in acute carbon monoxide poisoning. It is not known if the reduced blood oxygen content in smokers who develop sudden illness within a few hours of smoking cigarettes has any effect on clinical outcomes, or if heavy smokers might benefit from a slightly higher target saturation range than non-smokers during the first few hours of a serious illness in an effort to maintain a similar blood oxygen content.

Skin pigmentation may also influence the accuracy of pulse oximetry readings (usually overestimation but sometimes underestimation). In particular, the accuracy of pulse oximetry is impaired in dark skinned participants at saturation levels below 80-85\%. ${ }^{184-186}$ However, this should rarely be a problem in clinical practice if the saturation is maintained in the range suggested in the present guideline (94-98\% for most patients), although the work of Jubran and Tobin ${ }^{31}$ on ventilated participants suggested that an oxygen saturation of $92 \%$ was useful in predicting a $\mathrm{PaO}_{2}$ above $60 \mathrm{~mm} \mathrm{Hg}(8 \mathrm{kPa})$ in ventilated white participants but was less reliable in ventilated black participants who sometimes had an $\mathrm{SpO}_{2}$ reading that was more than 4\% above the directly measured $\mathrm{PaO}_{2}$. In the case of sickle cell crisis, pulse oximetry may underestimate the level of oxygenation. ${ }^{187}$ In these circumstances, under-reading is safer than over-reading because no truly hypoxaemic patient would be denied oxygen therapy. In sickle cell patients, one study has found that pulse oximeters did not misdiagnose either hypoxaemia or normoxaemia during a sickle cell crisis provided a good wave signal was present, ${ }^{188}$ but discrepancy between $\mathrm{SpO}_{2}$ and that measured by co-oximetry $\left(\mathrm{SO}_{2}\right)$ has been found by others during a sickle cell crisis ${ }^{189}$ and stable patients. ${ }^{190}$ In these studies, the $\mathrm{SpO}_{2}$ was generally lower than the $\mathrm{SaO}_{2}$, so pulse oximetry may misdiagnose hypoxaemia in sickle cell patients when none is present.

Oximeters can be affected by motion of the patient's hand, but this is less of a problem with modern oximeters than with older devices. ${ }^{191}$ Motion artefact is more of a problem if the patient also has reduced perfusion of the measuring site. ${ }^{192} \mathrm{~A}$ malpositioned oximeter sensor can cause artefact which can overestimate or underestimate the true oxygen saturation; this can be a particular problem during repositioning of ill patients. ${ }^{193}$

The site of oximetry is also important. Finger and earlobe measurements are more accurate than measurements from a probe applied to the toe, and finger probes may be more accurate than ear probes. ${ }^{194} 195$ Finally, clinical staff need to remember to remove nail varnish and false nails to avoid artefacts in oximetry measurements.

Evidence statements

- Pulse oximeters are accurate to within $1-2 \%$ of directly measured $\mathrm{SaO}_{2}$ in most participants but the error (usually overestimation but sometimes underestimation) is greater in dark skinned participants, especially with very low saturation (below 80-85\%) (evidence level 2+).

- The accuracy of oximeters in shock, sepsis and hypotension is largely unknown, but most errors are likely to result in falsely low readings which would result in additional oxygen being given. Most errors in oximetry are therefore not likely to place patients at risk, but it is important to ensure that the oximeter has a good signal and to avoid artefact due to motion, nail varnish or other potential sources of error (evidence level 3).

- It is advised that oximetry measurements on sleeping patients should be recorded over several minutes to avoid the possibility of being misled by a normal transient nocturnal dip in oxygen saturation and clinicians should look for evidence of snoring or sleep apnoea if desaturation is noted during sleep (evidence level 4).

- Pulse oximetry can be misleadingly normal in smokers because of raised blood carboxyhaemoglobin levels which will cause a reduced blood oxygen content despite an apparently normal oxygen saturation and a normal $\mathrm{PO}_{2}$. Patients who have smoked cigarettes in the previous 10 hours may therefore be at increased risk from hypoxaemia (evidence level 3).

- There are no controlled trials regarding implementation of these observational studies and the following recommendations are based on expert opinion (evidence level 4). 


\section{Recommendations}

B2: Oxygen saturation, 'the fifth vital sign', should be checked by trained staff using pulse oximetry in all breathless and acutely ill patients (supplemented by blood gases when necessary) and the inspired oxygen device and flow rate should be recorded on the observation chart with the oximetry result (grade D).

B3: Initial clinical assessment and subsequent monitoring of acutely unwell patients should include the use of a recognised physiological 'track and trigger' system, such as the NEWS which may trigger clinical review due to hypoxaemia, need for supplementary oxygen or for other reasons (grade D). BTS

B4: For patients who are at risk of hypercapnic respiratory failure, it is recommended that the relevant section of the 2017 NEWS chart should be used. Points are awarded if the oxygen saturation is below or above the target range (grade D).

\subsubsection{Arterial and arterialised blood gases}

$\mathrm{ABGs}$ are the 'gold standard test' for assessing respiratory failure. However, published studies have shown that arterialised capillary gases from the earlobe (but not from the finger) can provide an assessment of $\mathrm{pH}$ and $\mathrm{PCO}_{2}$ that is almost identical to that obtained from an arterial sample (indications for blood gas sampling are given in section 8.4 and recommendation 13). ${ }^{196-200}$ In acute and stable situations the earlobe specimen gives a $\mathrm{PO}_{2}$ measurement which is $0.5-1 \mathrm{kPa}$ (3.7$7.5 \mathrm{~mm} \mathrm{Hg}$ ) lower than the simultaneous arterial measurement with most of the divergence occurring at $\mathrm{PO}_{2}$ above $8-10 \mathrm{kPa}$ $(60-75 \mathrm{~mm} \mathrm{Hg}) \cdot{ }^{197}{ }^{200}$ This means that most patients can be managed safely based on the $\mathrm{pH}$ and $\mathrm{PCO}_{2}$ levels measured from earlobe blood gases supplemented by oxygen saturation measured by a pulse oximeter. ${ }^{197}{ }^{200}$ In critically ill patients, the initial specimen should be an arterial specimen to guarantee an accurate initial assessment, but capillary gases are especially valuable for monitoring progress of the blood gases as a patient stabilises.

Patients who have had simultaneous arterial and earlobe samples rated the earlobe puncture procedure as being considerably less painful than arterial puncture. ${ }^{201}$ However, the administration of local anaesthesia before ABG sampling produced a significant reduction in pain. ${ }^{202} 203 \mathrm{~A}$ recent randomised trial found that arterial punctures using insulin needles cause less pain and fewer procedural complications compared with standard needles. ${ }^{204}$

The pain score measured on a $100 \mathrm{~mm}$ visual analogue scale mean $( \pm S D)$ in punctures with the insulin needle was lower than the standard needle $(23 \pm 22$ vs $39 \pm 24 \mathrm{~mm}$; mean difference $=-15 \mathrm{~mm} ; 95 \%$ CI $-22 \mathrm{~mm}$ to $-7 \mathrm{~mm} ; \mathrm{p}<0.001)$. However, due to the higher rate of haemolysis, the authors recommended that the use of insulin needles should be limited to conditions that do not require a concurrent potassium value in the same blood sample.

There is a very small risk of arterial damage from arterial puncture, especially if the radial site is used. Most reports of hand ischaemia have involved indwelling radial artery cannulae, but the vessel could also be injured by needle puncture. ${ }^{205}$ The guideline therefore recommends that arterialised earlobe specimens should be used more widely than at present as a safer and less painful alternative to ABG sampling and local anaesthetic should be used wherever possible for ABG sampling, but this is often not practical in medical emergencies and blood gas sampling should not be delayed in these circumstances. However, the accuracy of earlobe samples in shock or hypotension is not known and it is recommended that ABGs should be used in all cases of shock or hypotension (systolic blood pressure $<90 \mathrm{~mm} \mathrm{Hg}$ ).

The technique of patient preparation, sample acquisition and sample processing for arterialised capillary gases is complex and should only be undertaken by fully trained staff. Capillary gases are very vulnerable to errors in technique and they should only be implemented for emergency use in units where staff have been fully trained in their use. See section 7.2.3 for advice concerning venous blood gases.

Evidence statements for use of arterialised capillary blood gas measurements

- Patients find earlobe specimens less painful than arterial puncture without local anaesthesia (evidence level $2++$ ).

- ABG sampling causes pain which can be reduced by the use of local anaesthetic prior to the test (evidence level $1+$ ).

- Arterialised earlobe blood gases will provide accurate information about $\mathrm{PaCO}_{2}$ and $\mathrm{pH}$ but do not provide accurate information concerning $\mathrm{PO}_{2}$ (evidence level 1-).

- The earlobe specimen gives a $\mathrm{PO}_{2}$ measurement which is $0.5-1 \mathrm{kPa}(4-7.5 \mathrm{~mm} \mathrm{Hg})$ lower than the simultaneous arterial measurement with greater divergence at oxygen levels above 8-10 kPa (60-75 mm Hg) (evidence level 1-).

- However, a combination of earlobe gases (to monitor $\mathrm{pH}$ and $\mathrm{PCO}_{2}$ ) and oximetry (to measure oxygen levels) will allow safe management of most patients, even in emergency settings. (The only published evidence is for patients with COPD but this finding is likely to be generalisable to most patients other than those with shock or poor peripheral circulation) (evidence level 4).

- The technique of patient preparation, sample acquisition and sample processing for arterialised capillary gases is complex and should only be undertaken by fully trained staff (evidence level 4).

Recommendations for use of ABGs and arterialised capillary blood gases

C1: For critically ill patients or those with shock or hypotension (systolic blood pressure $<90 \mathrm{~mm} \mathrm{Hg}$ ), the initial blood gas measurement should be obtained from an arterial sample. For most patients who require blood gas sampling, either ABGs or arterialised earlobe blood gases may be used to obtain an accurate measure of $\mathrm{pH}$ and $\mathrm{PCO}_{2}$. However, the $\mathrm{PO}_{2}$ is less accurate in earlobe blood gas samples (it underestimates the $\mathrm{PO}_{2}$ by $0.5-$ $1 \mathrm{kPa}$ ) so oximetry should be monitored carefully if earlobe blood gas specimens are used and a repeat arterial specimen should be taken if there is any concern about the accuracy of a capillary sample (grade D).

C2: Local anaesthesia should be used for all ABG specimens except in emergencies (grade A).

\subsubsection{Transcutaneous oxygen assessments}

Transcutaneous oxygen devices give different information from pulse oximetry. They are more sensitive to reduced perfusion and may be used to monitor tissue oxygenation in trauma patients but their use is beyond the scope of this guideline. ${ }^{206}$

\subsection{Assessment of hypercapnia and acidosis}

\subsubsection{Clinical assessment}

In patients with lung disease, hypercapnia may be accompanied by visible respiratory distress, but this will be absent when hypercapnia is a consequence of a reduction in minute ventilation. Patients may have a flushed face, a full and bounding pulse and muscle twitching together with the characteristic flap of the outstretched hands. In severe cases, consciousness may be depressed and convulsions may occur. 
Gross hypercapnia usually occurs with profound hypoxaemia and it is therefore difficult to disentangle the direct effect of hypercapnia per se. Coma will usually occur when the $\mathrm{PaCO}_{2}$ is in the range $12-16 \mathrm{kPa}(90-120 \mathrm{~mm} \mathrm{Hg})$. Survival has been seen following a $\mathrm{PaCO}_{2}$ of $67 \mathrm{kPa}(500 \mathrm{~mm} \mathrm{Hg}) .^{207}$

The presence of hypercapnic respiratory failure can be anticipated in patients with severe exacerbations of COPD or other diseases such as severe neuromuscular disorders. Carbon dioxide is a vasodilator so patients with hypercapnia may develop headache. Carbon dioxide in high concentrations has hypnotic effects and patients with hypercapnia may progress from drowsiness with flapping tremor to confusion to coma. ${ }^{84} 130-134$ A study of 127 episodes of acute respiratory acidosis showed that the best clinical predictors of respiratory acidosis were drowsiness (OR 7.1), flushing (OR 4.1), the presence of known COPD (OR 3.3) and the presence of intercostal retraction (OR 2.9). ${ }^{208}$

Clinical symptoms and signs of carbon dioxide retention include:

Headache.

Poor appetite.

Vasodilation producing flushing and warm peripheries with dilated blood vessels (including retinal veins).

Bounding pulse.

Drowsiness.

Flapping tremor.

Confusion.

Coma.

\subsubsection{Blood arterial and arteriolar gases}

Arterial or arterialised earlobe capillary blood gases will give an accurate estimation of $\mathrm{pH}$ and $\mathrm{PaCO}_{2}$ (see section 7.1 .3 for further details). ${ }^{196-198}$ The blood gases will need to be repeated in 30-60 min in patients with significant hypercapnia or acidosis to monitor the response to treatment. Patients with COPD who remain acidotic despite $30-60 \mathrm{~min}$ of standard treatment (including targeted low-concentration oxygen therapy) are likely to need NIV. ${ }^{209}$

\subsubsection{Venous $\mathrm{PCO}_{2}$ sampling}

It has been suggested that the venous $\mathrm{PCO}_{2}$ level can be used to screen for hypercapnia in patients with acute respiratory disease. A study of 196 paired samples of arterial and venous blood from patients with acute respiratory disease showed that the $\mathrm{PCO}_{2}$ in the venous sample was an average of $0.77 \mathrm{kPa}$ $(5.8 \mathrm{~mm} \mathrm{Hg})$ higher than the simultaneous arterial sample. ${ }^{210} \mathrm{~A}$ venous $\mathrm{PCO}_{2}$ below $6 \mathrm{kPa}(45 \mathrm{~mm} \mathrm{Hg})$ had $100 \%$ sensitivity for eliminating the risk of hypercapnia (arterial $\mathrm{PCO}_{2}$ above $6 \mathrm{kPa}$ or $45 \mathrm{~mm} \mathrm{Hg}$ ), although the specificity was low at $57 \%$ and there was more variation in some studies. ${ }^{211-213}$ For patients who are not at risk of metabolic acidosis, the presence of a satisfactory oxygen saturation measured by pulse oximetry and a venous $\mathrm{PCO}_{2}$ below $6 \mathrm{kPa}(45 \mathrm{~mm} \mathrm{Hg})$ can exclude the possibility of significant arterial hypoxia or hypercapnia and may obviate the need for ABG measurements. This advice is strengthened by a recent prospective cohort study and a meta-analysis of five previous studies. ${ }^{214} 215$ Although the use of venous $\mathrm{PCO}_{2}$ is becoming more common, it is still not widely enough used in clinical practice for the guideline committee to make a clear recommendation on its use.

\subsubsection{Carbon dioxide monitors and non-invasive assessments of hypercapnia}

Waveform capnography to assess end-tidal $\mathrm{CO}_{2}$ is used primarily to confirm tracheal intubation during anaesthesia, intensive care and for any patients requiring endotracheal intubation. They are considered the 'gold standard' by the Royal College of Anaesthetists. The absence of any detectable carbon dioxide output indicates a failed intubation. The management of intubated patients is outside the remit of this guideline.

Waveform capnography monitors are also useful in the management of cardiac arrest and circulatory collapse. Waveform capnography during CPR allows: (1) confirmation of correct tracheal tube placement; (2) monitoring of the respiratory rate; (3) assessment of CPR quality-improved chest compression quality results in increased end-tidal CO2 level; (4) indication of return of spontaneous circulation (ROSC) - a large increase in end-tidal $\mathrm{CO}_{2}$ during CPR can indicate ROSC; (5) a very low end-tidal $\mathrm{CO}_{2}$ value during $\mathrm{CPR}$ despite high-quality chest compressions is associated with a low probability of ROSC. ${ }^{216-218}$ These devices are also useful in the care of intubated patients in the emergency department because, through visualising a typical 'box wave form', they can confirm that the tube is in the airway even in the absence of carbon dioxide production during a cardiac arrest. A sudden increase in carbon dioxide may be the first sign of spontaneous circulation. ${ }^{219}$

End-tidal carbon dioxide measurements correlate poorly with arterial carbon dioxide levels in patients with COPD, but they may be useful in some research studies of hyperventilation syndromes. However, these devices are inaccurate in patients with airways disease and those with a high respiratory rate, so they should not be used in the management of patients with respiratory failure and they will not be discussed further in this guideline.

The recent availability of portable devices to measure transcutaneous partial pressure of carbon dioxide $\left(\mathrm{PtCO}_{2}\right)$ at the bedside provide a promising alternative to an ABG. They function on the principle that $\mathrm{CO}_{2}$ diffuses extremely well through tissues. A probe is attached to an area of skin (usually the earlobe) and warms to $42^{\circ} \mathrm{C}$ which 'arterialises' the underlying capillaries. The $\mathrm{CO}_{2}$ diffusing through the skin changes the $\mathrm{pH}$ of a thin electrolyte membrane in the probe and the resulting signal is converted to an estimate of the $\mathrm{PaCO}_{2}$. As well as measuring $\mathrm{PtCO}_{2}$, the probe also measure $\mathrm{SpO}_{2}$. Early studies indicate that such devices can be accurate in normal volunteers. Transcutaneous $\mathrm{CO}_{2}$ devices have provided accurate estimates of $\mathrm{PaCO}_{2}$ in a variety of clinical scenarios including AECOPD, invasive and NIV in ICUs and overnight studies of sleep-disordered breathing. ${ }^{220-224}$

Recently transcutaneous $\mathrm{CO}_{2}$ monitoring has been used in the emergency department in patients presenting with acute severe asthma ${ }^{136}$ and community-acquired pneumonia ${ }^{225}$ The $\mathrm{PtCO}_{2}$ accurately assessed $\mathrm{PaCO}_{2}$ as measured by the 'gold standard' $\mathrm{ABG} .{ }^{226} \mathrm{~A}$ rise in $\mathrm{PtCO}_{2}$ was demonstrated in both groups of patients on administration of high-flow oxygen for $60 \mathrm{~min}$, the significance of this will be discussed further in section 8.12. These new techniques are not yet recommended for routine clinical use.

\section{SECTION 8: EMERGENCY OXYGEN USE IN HOSPITAL SETTINGS}

Tables 1-4 are based on section 8, they are shown at the front of the guideline.

The hospital management of hypoxaemic patients is presented before the prehospital management. Some readers may prefer to read section 9 (prehospital care) first because most patients receive prehospital care before hospital care, but the Guideline Development Group preferred to present the hospital management first because a wider range of therapeutic options and a fuller range of investigations such as blood gas results are available in the hospital environment than in most prehospital settings. 


\subsection{Assessment and immediate management of breathless patients on arrival in hospital}

Breathless patients may arrive in hospital directly (without prior assessment) or in ambulances where they will usually have been assessed by paramedics who may also have initiated emergency treatments including oxygen therapy. As discussed in section 7 of this guideline, assessment, triage and resuscitation of critically ill patients must be undertaken in parallel with the initiation of oxygen therapy and specific treatment must be given for the underlying medical condition. All critically ill patients and all patients at risk of hypercapnic respiratory failure should be triaged as very urgent and should have blood gases taken on arrival in hospital. Furthermore, all seriously ill patients should be assessed by senior clinicians in the emergency department as early as possible. In many cases, this may involve liaison with intensive care specialists or with appropriate other specialists who can deal effectively with the patient's major medical or surgical problems.

- Readers are referred to section 7.1.1 and to disease-specific guidelines for advice concerning the immediate assessment and management of seriously ill patients.

- Readers are referred to section 10 for advice concerning choice of oxygen delivery devices and systems.

- Readers are referred to tables 1-4 and charts 1 and 2 in the introductory section for a summary of the key elements of oxygen therapy in common medical emergencies.

- Remember to ask for senior and specialist advice early in the care of profoundly ill patients.

- Clinicians must bear in mind that supplemental oxygen is given to improve oxygenation, but it does not treat the underlying causes of hypoxaemia which must be diagnosed and treated as a matter of urgency.

\subsection{Differences in management in hospital compared with a prehospital setting}

The immediate management of medical emergencies in hospital settings before blood gas results are available is similar in principle to management in the prehospital setting (section 9). The main priorities are to avoid harmful levels of hypoxaemia for all patients and to avoid harmful levels of hypercapnia for patients who are at risk of this complication. However, the amount of information available to the healthcare professionals increases rapidly in the hospital environment. The hospital management is presented before the prehospital management because it represents the 'ideal' management. This may also be achievable in some prehospital settings such as a well-equipped primary care centre and in some ambulance settings such as ambulances with critical care outreach facilities. However, in many prehospital settings there will usually be less information available concerning a patient's history and physiology and less equipment available to assess and treat the patient.

Differences between hospital settings and prehospital settings include:

- Pulse oximetry is almost always available in hospital at present. These guidelines also recommend that pulse oximetry must be available in all locations where emergency oxygen is used (section 9.1).

- Blood gas results can be available within minutes of arrival in hospital.

- Additional diagnostic information may be available from history, clinical examination, test results and from the patient's hospital records.

- Additional equipment and resources are available.

Because of the universal availability of oximetry in hospitals, it is rare for the hospital medical team to have to administer oxygen on the basis that a patient 'might be hypoxaemic'. However, initial 'blind management' is sometimes necessary for patients with shock or with very poor peripheral circulation where a reliable pulse oximetry trace cannot be obtained. ABGs should be obtained as a matter of urgency in all such cases.

\subsection{Which patients need oxygen therapy?}

Supplemental oxygen therapy is recommended for all acutely hypoxaemic patients and for many other patients who are at risk of hypoxaemia, including patients with major trauma and shock. Many acutely breathless patients will require supplemental oxygen therapy, but there are some situations such as acute hyperventilation or diabetic ketoacidosis where an apparently breathless patient will not benefit from oxygen therapy. There are some other clinical situations such as carbon monoxide or cyanide poisoning where a patient may benefit from oxygen therapy despite an apparent lack of hypoxaemia or breathlessness because carbon monoxide binds more avidly than oxygen to the haemoglobin molecule and cyanide poisoning leads to histotoxic hypoxia. Carbon monoxide poisoning and cyanide poisoning often coexist in patients suffering from smoke inhalation. ${ }^{227}$

Although it is well recognised that profound hypoxaemia can lead to organ failure and death, it would be unethical to undertake randomised studies involving the deliberate exposure of acutely ill patients to severe hypoxaemia so most recommendations are based on expert opinion unless otherwise stated.

Oxygen saturation should be measured in all breathless and acutely ill patients as discussed in recommendation 9 and oxygen therapy should be given to hypoxaemic patients as described in tables 1-4 and recommendations 1-4. Patients do not require oxygen therapy if their oxygen saturation is $94 \%$ or above (exceptions are carbon monoxide poisoning, cyanide poisoning and pneumothorax; see sections 8.10.7 and 8.11.6). Patients on oxygen with $\mathrm{SpO}_{2}>98 \%$ (with the above exceptions) do not usually require oxygen therapy or may require a lower concentration. All patients with shock, major trauma, sepsis or other critical illness should be managed initially with high-concentration oxygen therapy from a reservoir mask. Targeted oxygen therapy can be initiated once there is spontaneous circulation and oximetry can be measured reliably (see table 1 and recommendations E1-E2).

\subsection{Which patients require blood gas measurements?}

Blood gases should be measured as soon as possible in most emergency situations involving hypoxaemic patients ${ }^{228}$ and are essential in patients who may develop hypercapnic respiratory failure (carbon dioxide retention with risk of respiratory acidosis). This includes all patients admitted with an AECOPD, even in the absence of hypoxaemia. ${ }^{1}$ Blood gases should also be checked (and the clinical situation should be reviewed) if the oxygen saturation should fall by more than three percentage points, even if the saturation remains within the target range. For example, a fall from $98 \%$ to $94 \%$ might be due to a significant event such as a pulmonary embolus. In this situation, the saturation of $94 \%$ will not harm the patient but the patient will remain at serious risk until the pulmonary embolism is diagnosed and treated. If oximetry shows a patient to be hypoxaemic, the initiation of oxygen therapy should not be delayed while awaiting the results of blood gas measurements.

Blood gas measurements are not usually required for patients with no risk factors for hypercapnic respiratory failure and an oxygen saturation of $94 \%$ or above breathing air unless the patient requires blood gas estimation for other reasons such as suspected metabolic acidosis or diabetic ketoacidosis. The BTS 
asthma guideline recommends that ABG measurements need not be undertaken in patients with acute asthma and an oxygen saturation above $92 \%$ and no life-threatening features. ${ }^{22}$ ABG sampling can be technically difficult, especially for poorly perfused patients, and junior staff should ask for assistance from more senior staff in difficult cases.

Following initial clinical assessment and the availability of a pulse oximetry measurement, a decision can be made regarding the need for blood gas estimation within a few minutes of arrival in the hospital environment or if a previously stable patient develops breathlessness within a hospital environment. Oximetry will give no information concerning carbon dioxide or $\mathrm{pH}$ levels and a normal pulse oximetry level may provide false reassurance in patients on oxygen therapy who may have unexpected hypercapnia and acidosis. However, careful clinical assessment supplemented by the use of oximetry will allow the setting of an appropriate oxygen saturation target for different groups of patients until blood gas results are available.

If repeated blood gas estimations are required, the timing will depend on the indication. In general, the oxygen saturation (and $\mathrm{PO}_{2}$ ) stabilises at a new higher level within a few minutes of increasing the concentration of oxygen but the $\mathrm{PaCO}_{2}$ can take 30-60 min to equilibrate. The rise in blood oxygen level can be monitored with oximetry, so repeat blood gas tests are performed mostly to assess critical illness (immediate sampling required) or to monitor $\mathrm{pH}$ and $\mathrm{PCO}_{2}$ levels (best performed 30-60 min after increasing the concentration of oxygen).

Evidence statement:

- The use of blood gases in acute illness is guided by observational data and expert opinion (evidence level 4).

\section{Recommendation}

C3: Blood gases should be checked in the following situations

- All critically ill patients (grade D).

- Unexpected or inappropriate fall in $\mathrm{SpO}_{2}$ below 94\% in patients breathing air or oxygen or any patient requiring oxygen to achieve the above target range (grade D). (Allowance should be made for transient dips in saturation to $90 \%$ or less in normal participants during sleep.)

- Deteriorating oxygen saturation (fall of $\geq 3 \%$ ) or increasing breathlessness in a patient with previously stable chronic hypoxaemia (eg, severe COPD) (grade D).

- Most previously stable patients who deteriorate clinically and require increased $\mathrm{FiO}_{2}$ to maintain a constant oxygen saturation (grade D).

- Any patient with risk factors for hypercapnic respiratory failure who develops acute breathlessness, deteriorating oxygen saturation, drowsiness or other features of carbon dioxide retention (grade D).

- Patients with breathlessness who are thought to be at risk of metabolic conditions such as diabetic ketoacidosis or metabolic acidosis due to renal failure (grade D).

- Any other evidence from the patient's medical condition that would indicate that blood gas results would be useful in the patient's management (eg, an unexpected change in 'track and trigger' systems such as a sudden rise of several units in the NEWS or an unexpected fall in oxygen saturation of $3 \%$ or more, even if within the target range) (grade D).

\subsection{Can arterialised earlobe capillary gases be used as a substitute for ABGs?}

Readers are referred to section 7.1.3 for advice concerning when to use ABGs and when to use arterialised capillary blood gases.

\subsection{Should oxygen be prescribed at a fixed concentration or} to achieve a target saturation?

In the past, oxygen was prescribed at a fixed $\mathrm{FiO}_{2}$ or at a fixed flow rate via nasal cannulae or variable performance face masks. However, several audits have shown that many (or most) patients did not receive the prescribed concentration of oxygen when this methodology was used. ${ }^{4-10}$ Furthermore, a patient's oxygen requirement may vary over time so the prescribed oxygen concentration may be too high or too low even a short time after the prescription was written. For this reason, it is recommended that oxygen should be prescribed to a target saturation range rather than prescribing a fixed concentration of oxygen or $\mathrm{FiO}_{2}$. This is analogous to an insulin 'sliding scale' where the prescriber specifies a variable dose of insulin to achieve a target blood glucose range rather than prescribing a fixed dose of insulin. This will allow the appropriate healthcare professional-usually a doctor, registered nurse or physiotherapist- to adjust each patient's concentration of oxygen to achieve the safest oxygen saturation range for each patient.

The prescriber may indicate a starting concentration, device or flow rate, but there needs to be an agreed system for adjusting the oxygen concentration upwards or downwards according to a patient's needs (see charts 1 and 2; sections 11.3.6 and 11.3.7 and figure 19). As a patient improves, he or she is likely to require a lower $\mathrm{FiO}_{2}$ over a time period that will vary between patients. Most recovering patients will eventually require no supplemental oxygen. On the other hand, a deteriorating patient may need an increased concentration of oxygen. This increase can be initiated by registered nursing staff or physiotherapists.

Good practice points

$\checkmark$ The requirement for an increased concentration of oxygen is an indication for urgent clinical reassessment of the patient (and repeat blood gas measurements in most instances). See recommendations W13 and W15 for exceptions.

$\checkmark$ Oxygen should be prescribed to a target saturation range rather than prescribing a fixed concentration of oxygen or $\mathrm{FiO}_{2}$ (see recommendations A1, A2, A4 and A5).

It is recommended that all patients admitted to hospital should have a prescription for an oxygen target range set at the time of admission, ideally as part of an 'admission bundle' which may include other key elements such as thromboprophylaxis and MRSA prevention. This will ensure that the correct amount of oxygen will be administered while awaiting full medical assessment if the patient should deteriorate unexpectedly at any time during the hospital admission.

\subsection{What should be the target oxygen saturation range for patients receiving supplementary oxygen?}

\subsubsection{Oxygen saturation target range for most patients}

As discussed in sections 4-6 of this guideline, there is no evidence of benefit from above normal oxygen saturation in most clinical situations and there is evidence that excessive concentrations of oxygen can have adverse effects, even in some patients who are not at risk of hypercapnic respiratory failure. A target oxygen saturation range of $94-98 \%$ will achieve normal or near-normal oxygen saturation for most patients who are not at risk of hypercapnic respiratory failure. Furthermore, the suggested lower limit of $94 \%$ allows a wide margin of error in the oximeter measurement, thus minimising the risk of any patient being allowed to desaturate below $90 \%$ due to inaccurate oximetry. 
8.7.2 Oxygen requirements for specific groups of patients

- Patients with critical illness requiring high-concentration oxygen therapy are discussed in section 8.10.

- Patients with medical emergencies which frequently cause breathlessness and hypoxaemia are discussed in section 8.11.

- Patients with COPD and other conditions that may predispose to hypercapnic respiratory failure are discussed in section 8.12.

- Medical emergencies for which oxygen was commonly given in the past but is not actually indicated unless the patient is hypoxaemic are discussed in section 8.13 .

\subsection{Importance of blood gas measurements in guiding oxygen therapy}

As soon as blood gas measurements are available, a patient's further treatment can be guided by the results of this test. For patients with a normal or low $\mathrm{PCO}_{2}$ and no risk factors for hypercapnic respiratory failure, it is safe to aim at an oxygen saturation in the normal range (94-98\%). For patients with a raised $\mathrm{PCO}_{2}$, a lower oxygen saturation is indicated (88-92\%), especially if the patient is acidotic. NIV is recommended for patients with COPD who have hypercapnia and a $\mathrm{pH}<7.35$ $\left(\left[\mathrm{H}^{+}\right]>45 \mathrm{nmol} / \mathrm{L}\right)$ despite 1 hour of standard medical treatment including controlled oxygen therapy. ${ }^{1} 209$

\subsection{What should be the initial choice of oxygen delivery system in hospital settings?}

The technical and practical aspects of different oxygen delivery systems are discussed in section 10. For major trauma cases and for severely hypoxaemic patients without risk factors for hypercapnic respiratory failure, a non-rebreathing mask (reservoir mask) at $15 \mathrm{~L} / \mathrm{min}$ is the suggested first choice. The delivery system and $\mathrm{FiO}_{2}$ may be adjusted later to a lower concentration of oxygen as a patient improves or towards supported ventilation if the patient deteriorates. The majority of patients with modest hypoxaemia can be treated with nasal cannulae or a simple face mask at a flow rate which is adjusted to maintain the oxygen saturation in the target range for their specific clinical presentation. Chart 2 shows a suggested scheme that allows the oxygen level to be adjusted upwards or downwards in gradual increments depending on a patient's clinical progress (see also sections 11.3.6 and 11.3.7). Venturi masks were previously recommended for low-concentration oxygen therapy because they deliver a more reliable oxygen concentration than nasal cannulae or variable flow masks. ${ }^{230}$ They can also be combined with a humidifier system when necessary (see section 10.2.3). The mask and/or flow should be rapidly changed if the initial choice does not achieve the target saturation. However, the only randomised study of targeted oxygen therapy in AECOPD used nasal cannulae which have the advantage of being adjustable (by variation in flow rate) without having to change the device itself. $^{51}$

\subsubsection{Devices used in emergency oxygen therapy in hospitals} (see section 10 for further details)

- High-concentration oxygen from reservoir mask $(15 \mathrm{~L} / \mathrm{min})$ or when using a bag mask or advanced airways (supraglottic airway or tracheal tube) for assisted ventilation during respiratory arrest or during CPR.

- Nasal cannulae $(2-6 \mathrm{~L} / \mathrm{min})$ or simple face masks $(5-10 \mathrm{~L} /$ min) for medium-concentration oxygen therapy (and HFNC using specialised equipment for high-concentration oxygen therapy, often as an alternative to a reservoir mask).
- Twenty-four per cent Venturi mask at $2 \mathrm{~L} / \mathrm{min}$ or $28 \%$ Venturi masks at $4 \mathrm{~L} / \mathrm{min}$ for patients at risk of hypercapnic respiratory failure (alternatively use nasal cannulae at 1-2 L/ min, especially when the patient has stabilised).

- Tracheostomy masks for patients with a functioning tracheostomy (adjust flow to achieve desired saturation).

\subsection{Recommended oxygen therapy for major medical emergencies and critical illness (see also table 1 and recommendations E1-E6)}

There are a number of major medical emergencies where patients are very likely to suffer from hypoxaemia. Highconcentration oxygen therapy from a reservoir mask at $15 \mathrm{~L} / \mathrm{min}$ is recommended in the initial management of all such patients prior to stabilisation and the availability of a reliable oximetry reading. Once this is achieved, the concentration of oxygen can be titrated downwards to maintain a target saturation of 94-98\%. It is recommended that patients with COPD or other risk factors for hypercapnia who develop a critical illness should be treated by emergency services in the same manner as other critically ill patients until urgent blood gas results become available because the primary issue is the critical illness. Critically ill patients with hypercapnia, hypoxaemia and acidosis will require immediate assessment by intensive care teams and will usually require tracheal intubation and mechanical ventilation.

Evidence statement

- The use of oxygen in major medical emergencies is guided by observational data and expert opinion (evidence level 4).

\subsubsection{Cardiac arrest and other conditions requiring CPR}

The 2015 guideline for Adult Advanced Life Support issued by Resuscitation Council UK recommends the use of the highest possible inspired oxygen level for ventilation during CPR; that guideline also makes recommendations regarding choice of airway and ventilation technique in these circumstances. ${ }^{231}$ The present guideline endorses these proposals during the period of resuscitation. Subsequent management will depend on the underlying condition and the patient's degree of recovery. There is theoretical evidence that patients with a ROSC may be managed more safely with a lower initial inspired oxygen than with $100 \%$ oxygen. ${ }^{98} 99$ Recently, in an observational study, Kilgannon et al ${ }^{151} 152$ reported increased mortality among survivors of cardiac arrest who had been exposed to very high levels of oxygen $\left(\mathrm{PaO}_{2}>300 \mathrm{~mm} \mathrm{Hg}\right.$ or $\left.40 \mathrm{kPa}\right)$ in the first 24 hours on the ICU. Bellomo et $a l^{154}$ also found an excess of deaths in this group of patients but argued that the risk was no longer statistically significant after multivariate analysis. Elmer et al $^{232}$ reported a small single-centre study in 2015 in which severe hyperoxaemia $\left(\mathrm{PaO}_{2}>300 \mathrm{~mm} \mathrm{Hg}\right.$ or $\left.40 \mathrm{kPa}\right)$ was associated with increased hospital mortality but moderate hyperoxaemia (101-299 $\mathrm{mm} \mathrm{Hg}$ ) was not associated with decreased survival.

A systematic review and meta-analysis by Wang et $a l^{233}$ concluded that hyperoxaemia appears to be correlated with increased in-hospital mortality in survivors of adult cardiac arrest but the result should be interpreted cautiously because of the significant heterogeneity and the limited number of studies that were analysed.

Based on the above evidence, current UK and European Resuscitation guidelines recommend a target oxygen saturation of $94-98 \%$ once spontaneous circulation had been restored. ${ }^{231}$ This guideline endorses these recommendations. 


\section{Evidence statement}

- The use of oxygen during and after CPR is based on expert opinion and extrapolation from observational data (evidence level 4).

\section{Recommendation}

E1: Use the highest feasible inspired oxygen for ventilation during CPR (see table 1 and section 8.10). Once spontaneous circulation has returned and arterial blood oxygen saturation can be monitored reliably, aim for a target saturation range of $94-98 \%$ and take an ABG sample to guide ongoing oxygen therapy. If the blood gas shows hypercapnic respiratory failure, reset the target range to $88-92 \%$ or consider mechanical ventilation (grade D).

\subsubsection{Critically ill patients including major trauma, shock and major sepsis}

There is evidence that early intervention to normalise oxygen delivery to the tissues using volume expansion and vasoactive agents is beneficial in the management of critically ill patients with shock or sepsis, but there is no evidence of benefit from attempts to achieve supranormal oxygen delivery. ${ }^{73-75} 234-238$ In fact, there is evidence that hyperoxia can cause a paradoxical decrease in whole body oxygen consumption in critically ill patients, ${ }^{239}$ and it has been demonstrated that hyperoxia can impair oxygen delivery in septic patients. ${ }^{240}$ A study using a rat model of progressive haemorrhage found that both hypoxaemia and hyperoxaemia compromised haemodynamics. There was a paradoxical fall in tissue $\mathrm{PO}_{2}$ in hyperoxaemic animals despite the high $\mathrm{PaO}_{2}{ }^{241}$ These factors may account for the observation by deJonge (but disputed by Eastwood) that mortality was elevated among intensive care patients who were hyperoxaemic during the first day on the ICU. ${ }^{153}{ }^{155}$ In de Jonge's study of 36307 patients on ICUs in the Netherlands, the adjusted OR for death was lowest in a $\mathrm{PO}_{2}$ range of $8.9-10.6 \mathrm{kPa}$ (equivalent to saturation about $93-96 \%$ at normal $\mathrm{pH}$ ). Two recent systematic reviews with meta-analysis have addressed this subject. Both studies concluded that hyperoxaemia was associated with increased hospital mortality but caution is required in interpreting the results because of heterogeneity in the included studies, including different definitions of hyperoxaemia. ${ }^{242} 243$ A randomised trial published in 2016 reported ICU mortality of $11.6 \%$ in 216 patients randomised to a target saturation range of 9498\% (median $\mathrm{PaO}_{2} 11.6 \mathrm{kPa}$ ) compared with $20.2 \%$ mortality among 218 patients randomised to conventional therapy (median $\left.\mathrm{PaO}_{2} 13.6 \mathrm{kPa}\right){ }^{244}$ The absolute risk reduction was $8.6 \%$ (risk reduction $0.086 ; 95 \%$ CI 0.017 to $0.150 ; \mathrm{p}=0.01$ ) and hospital mortality was $24.2 \%$ vs $33.9 \%$ (absolute risk reduction $0.099 ; 95 \%$ CI 0.013 to $0.182 ; \mathrm{p}=0.03$ ). However, the trial was prematurely terminated due to difficulty with recruitment, and the authors advise caution in interpreting the results for this reason.

Most critically ill patients are at risk of multiorgan failure and therefore require intensive care assessment as a matter of urgency. Critical care consensus guidelines set $90 \%$ saturation as the minimum level below which oxygen saturation should not be allowed to fall. ${ }^{31} 32245$ The 2012 Surviving Sepsis Campaign guideline is mainly based on mixed venous oxygen saturation or central venous saturation but also recommends a target $\mathrm{SaO}_{2}$ of $88-95 \%$ for patients with sepsis. ${ }^{31} 32245$ However, these recommendations are based on directly measured $\mathrm{SaO}_{2}$ in critical care settings with intensive levels of nursing and monitoring. The present guideline recommends a slightly higher target saturation range prior to the transfer of these seriously ill patients to critical care facilities.

For most critically ill or severely hypoxaemic patients, initial oxygen therapy should involve the use of a reservoir mask, aiming at an oxygen saturation of 94-98\%. If the patient has concomitant COPD or other risk factors for hypercapnic respiratory failure, the initial saturation target should also be 94-98\% pending the results of blood gas estimations and assessment by intensive care specialists. If critically ill patients with COPD have hypercapnia and acidosis, the correction of hypoxaemia must be balanced against the risks of respiratory acidosis and ventilatory support using NIV or invasive ventilation should be considered.

It is also recognised that many patients with long bone fractures may develop hypoxaemia even in the absence of injury to the airway or chest (possibly due to opioid treatment and fat embolism) and they should be monitored with oximetry and given oxygen if necessary. ${ }^{38} 246-248$ These patients, if not critically ill, should have a target oxygen saturation of $94-98 \%$ or $88-92 \%$ if they have coexisting COPD or other risk factors for hypercapnic respiratory failure.

Evidence statement

- The use of oxygen in critical illness is based on expert opinion guided by physiology and observational data (evidence level 4).

\section{Recommendation}

E2: In critical illness, including major trauma, sepsis, shock and anaphylaxis, initiate treatment with a reservoir mask at $15 \mathrm{~L} / \mathrm{min}$ and aim at a saturation range of 94-98\%. This advice also applies to patients with critical illness who have risk factors for hypercapnia pending the results of blood gas measurements and expert assessment. In patients with spontaneous circulation and a reliable oximetry reading, it may be possible to maintain a saturation of $\mathbf{9 4 - 9 8 \%}$ using lower concentrations of oxygen (grade D).

\subsubsection{Drowning}

Survivors of drowning may have suffered inhalation of fresh or sea water into the lungs and may become hypoxaemic. Supplemental oxygen should be given to all patients with saturation below $94 \%$, aiming at a target saturation of $94-98 \%{ }^{249}$

Evidence statement

- The use of oxygen in drowning is based on expert opinion (evidence level 4).

\section{Recommendation}

E3: In cases of drowning, aim at an oxygen saturation of $94-98 \%$ once adequate circulation is restored (grade D).

\subsubsection{Anaphylaxis (see recommendation E2)}

Patients with anaphylaxis are likely to suffer from tissue hypoxia due to a combination of upper and/or lower airway obstruction together with hypotension. In addition to specific treatment of these problems, the Resuscitation Council UK recommends initial high-concentration oxygen $(10-15 \mathrm{~L} / \mathrm{min}$, by reservoir mask) for patients with anaphylaxis. ${ }^{231} 250$ The present guideline would endorse this practice in the immediate management of anaphylaxis followed by a target saturation of $94-98 \%$ once the patient's oxygen saturation can be measured reliably.

Evidence statement

- The use of oxygen in anaphylaxis is based on expert opinion (evidence level 4).

\subsubsection{Major pulmonary haemorrhage or massive haemoptysis}

Major pulmonary haemorrhage and massive haemoptysis can occur for a large number of reasons ranging from acute pulmonary vasculitis to erosion of a blood vessel by a lung tumour. In addition to specific treatment of the causative condition, most such patients require supplemental oxygen treatment (also see 
table 1). A target saturation range of $94-98 \%$ is recommended. Treatment should be initiated with high-concentration oxygen via a reservoir mask and subsequently adjusted according to chart 2 to maintain a saturation of $94-98 \%$ pending the results of blood gas measurements.

Evidence statement

- The use of oxygen in pulmonary haemorrhage is based on expert opinion (evidence level 4).

\subsubsection{Epileptic fits}

As this is a life-threatening condition where a patient may suffer from cerebral hypoxia (and oximetry may not be possible), patients with status epilepticus should be treated in accordance with table 1 (reservoir mask until clinically stabilised).

\section{Evidence statement}

- There have been no clinical trials of oxygen use during epileptic fits and it may be impossible to obtain an oximetry signal in these circumstances. Expert opinion advises the administration of high-concentration oxygen until oximetry measurements can be obtained (evidence level 4).

\section{Recommendation}

E4: In patients with acute seizures due to epilepsy or other causes, high-concentration oxygen should be administered until a satisfactory oximetry measurement can be obtained and clinicians should then aim for an oxygen saturation of $94-98 \%$ or $88-92 \%$ if the patient is at risk of hypercapnic respiratory failure (grade D).

\subsubsection{Major head injury}

Patients with major head injury are at risk of hypoxaemia and hypercapnia. They require urgent assessment and maintenance of airway patency, either through positioning, simple adjuncts or early tracheal intubation and ventilation to avoid further brain injury due to brain oedema which may be aggravated by hypoxaemia and/or hypercapnia. These patients should be triaged directly where possible to a major trauma centre. Initial treatment should include high-concentration oxygen via a reservoir mask pending availability of satisfactory blood gas measurements or until the airway is secured by intubation which will also protect against the risk of aspiration due to reduced consciousness. Although hypoxaemia is common in patients with head injury (and associated with poorer outcomes), the relative contribution of hypoxaemia to outcome is not yet established. ${ }^{37}$ 251-255 All authors agree that hypoxaemia should be corrected, but a review of the literature concluded that there is no evidence of clinical benefit from hyperoxia in brain injured patients and a subsequent clinical study showed that normobaric hyperoxia did not improve brain metabolism in five patients with acute severe brain injury. 256257 There are no UK guidelines for oxygen therapy in the immediate phase after head injury, but US guidelines recommend maintaining an oxygen saturation above $90 \%$ for patients with acute brain injury. ${ }^{30}$ An American study published in 2009 describes a cohort of 3420 patients and the authors reported worse outcomes in patients with severe traumatic brain injury associated with hypoxaemia and extreme hyperoxaemia. Outcomes were optimal in the $\mathrm{PaO}_{2}$ range of $110-487 \mathrm{~mm} \mathrm{Hg}(14.6-65 \mathrm{kPa}){ }^{258}$ Other observational studies have suggested that hyperoxaemia may be associated with improved outcomes. Asher et al ${ }^{259}$ recently reported the outcomes for 193 adult patients with severe traumatic brain injury who had overall survival of $57 \%$. They reported that a $\mathrm{PaO}_{2}$ threshold between 250 and $486 \mathrm{~mm} \mathrm{Hg}(33-65 \mathrm{kPa})$ during the first 72 hours after injury was associated with improved all-cause survival, independent of hypocarbia or hypercarbia. Two recent observational studies have provided further evidence that hyperoxaemia may be associated with increased mortality in traumatic brain injury. ${ }^{260} 261$ The present guideline advises giving supplementary oxygen if required to maintain an oxygen saturation in the range of $94-98 \%$ pending the publication of randomised studies comparing normoxaemia with normobaric hyperoxaemia.

Evidence statement

- The use of oxygen in cases of major head injury is based on expert opinion and extrapolation from observational studies (evidence level 4).

\section{Recommendation}

E5: In cases of major head injury, aim at an oxygen saturation of $94-98 \%$. Initial treatment should involve highconcentration oxygen from a reservoir mask at $15 \mathrm{~L} / \mathrm{min}$ pending availability of satisfactory blood gas measurements or until the airway is secured by intubation (grade $\mathrm{D}$ ).

\subsubsection{Carbon monoxide poisoning}

Patients with carbon monoxide poisoning may have a normal level of $\mathrm{PaO}_{2}$ but a greatly reduced level of oxygen bound to haemoglobin because this has been displaced by carbon monoxide. ${ }^{262}$ Standard pulse oximetry cannot screen for carbon monoxide exposure as it does not differentiate carboxyhaemoglobin from oxyhaemoglobin and blood gas measurements will show a normal $\mathrm{PaO}_{2}$ in these patients. Some newer co-oximeters can measure carboxyhaemoglobin levels as well as $\mathrm{SpO}_{2}$. The blood carboxyhaemoglobin level must be measured to assess the degree of carbon monoxide poisoning. The half-life of carboxyhaemoglobin in a patient breathing air is $\sim 300 \mathrm{~min}$; this decreases to $90 \mathrm{~min}$ with high-concentration oxygen via a reservoir mask. The most important treatment for a patient with carbon monoxide poisoning is therefore to give highconcentration oxygen via a reservoir mask. Comatose patients or those with severe mental impairment should be intubated and ventilated with $100 \%$ oxygen. The role of hyperbaric oxygen remains controversial. A 2011 Cochrane review concluded that existing randomised trials did not establish whether the administration of hyperbaric oxygen to patients with carbon monoxide poisoning reduced the incidence of adverse neurological outcomes. ${ }^{263}$ However, a randomised trial published in 2007 has suggested that patients with loss of consciousness or high carboxyhaemoglobin levels may have less cognitive sequelae if given hyperbaric oxygen. ${ }^{264}$

Evidence statement

- The use of oxygen in carbon monoxide poisoning is based on expert opinion and extrapolation from observational studies (evidence level 4).

\section{Recommendation}

E6: In cases of carbon monoxide poisoning, an apparently 'normal' oximetry reading may be produced by carboxyhaemoglobin, so aim at an oxygen saturation of $100 \%$ and use a reservoir mask at $15 \mathrm{~L} / \mathrm{min}$ irrespective of the oximeter reading and $\mathrm{PaO}_{2}$ (grade $\mathrm{D}$ ).

\subsection{Serious illnesses requiring moderate levels of supplemental oxygen if the patient is hypoxaemic (see table 2)} Patients who present with acute medical emergencies who are not critically ill and who have saturation $>85 \%$ can be treated with medium-concentration oxygen therapy from nasal cannulae or a simple face mask with a target saturation range of $94-98 \%$. Some of these patients (eg, patients with pneumonia) may subsequently deteriorate, requiring high-concentration oxygen from a 
reservoir mask or requiring respiratory support such as invasive ventilation. Others may turn out to have an additional diagnosis of COPD or neuromuscular disease with a risk of hypercapnic respiratory failure and they should be managed with a $24 \%$ Venturi mask at $2-3 \mathrm{~L} / \mathrm{min}$ or $28 \%$ Venturi mask at $4 \mathrm{~L} / \mathrm{min}$ or 1-2 L of oxygen via nasal cannulae, aiming at a target saturation of $88-92 \%$. There are no published trials supporting the use of oxygen to relieve breathlessness in non-hypoxaemic patients, and there is evidence from randomised studies that oxygen does not relieve breathlessness compared with air in non-hypoxaemic patients with COPD who are breathless following exertion or breathless palliative care patients with $\mathrm{PO}_{2}>7.3 \mathrm{kPa}$ or $55 \mathrm{~mm} \mathrm{Hg}$ (saturation above about 88\%). ${ }^{146} 150$

\subsubsection{Patients with acute onset of hypoxaemia of unknown} cause with no pre-existing respiratory disorders or risk factors It is common for breathless and hypoxaemic patients to have no firm diagnosis at the time of presentation. For most acutely hypoxaemic patients whose medical problem is not yet diagnosed, an oxygen saturation range of $94-98 \%$ will avoid the potential hazards associated with hypoxaemia or hyperoxaemia (see sections 4-6 and tables 1-2). Aiming for an oxygen saturation in the normal range (rather than an abnormally high oxygen level) will also have the effect of allowing the lowest effective $\mathrm{FiO}_{2}$ to be used, thus avoiding risks such as absorption atelectasis and V/Q mismatch that may be associated with the use of very high fractions of inspired oxygen (see sections 5 and 6). The priority for such patients is to make a specific diagnosis as early as possible and to institute specific treatment for the underlying condition. Early blood gas measurement is mandatory in the management of patients with sudden unexplained hypoxaemia.

Evidence statement

- The use of oxygen in most instances of acute hypoxaemia is guided by expert opinion based on physiology and observational studies except where indicated otherwise in the sections that follow (evidence level 4).

\section{Recommendations}

D1: For acutely breathless patients not at risk of hypercapnic respiratory failure who have saturations below $85 \%$, treatment should be started with a reservoir mask at $15 \mathrm{~L} / \mathrm{min}$ in the first instance (see figures 1-2 (charts 1-2) and table 2 and sections 8.10 and 10).* The oxygen concentration can be adjusted downwards (using nasal cannulae at $1-6 \mathrm{~L} / \mathrm{min}$ or a simple face mask at $5-10 \mathrm{~L} / \mathrm{min}$ ) to maintain a target saturation of $94-98 \%$ once the patient has stabilised (grade D).

D2: In other cases of acute hypoxaemia without critical illness or risk factors for hypercapnic respiratory failure, treatment should be started with nasal cannulae (or a simple face mask if cannulae are not tolerated or not effective) with the flow rate adjusted to achieve a saturation of $94-98 \%$ (grade D).

D3: If medium-concentration therapy with nasal cannulae or a simple face mask does not achieve the desired saturation, change to a reservoir mask and seek senior or specialist advice (grade D). Good practice point

$\checkmark$ High-flow nasal oxygen using specialised equipment should be considered as an alternative to reservoir mask treatment in patients with acute respiratory failure without hypercapnia.

*For initial management of patients at risk of hypercapnic respiratory failure, see recommendations G1 and G2.

\subsubsection{Acute asthma}

The BTS/SIGN guideline for the management of acute asthma recommends that the oxygen saturation should be maintained between $94 \%$ and $98 \% .^{229}$ Although there is no danger of tissue hypoxia at any saturation above 90\%, a drop of oxygen saturation below 94\% may indicate deterioration and should prompt a further assessment. Supplemental oxygen should be started using nasal cannulae at $2-4 \mathrm{~L} / \mathrm{min}$ or a simple face mask at $5 \mathrm{~L} / \mathrm{min}$ or $35-40 \%$ Venturi mask and adjusted as necessary to maintain a saturation of $94-98 \%$. $^{265}$

A study which was published in 2003 showed that the administration of $100 \%$ oxygen to patients with acute severe asthma produced an increased $\mathrm{PaCO}_{2}$ and a decreased peak expiratory flow compared with patients treated with $28 \%$ oxygen. $^{266}$ The authors of that study recommended the use of targeted oxygen therapy rather than giving high-concentration oxygen to all patients with acute severe asthma. This advice has been reinforced by the randomised study of Perrin et $a l^{136}$ who found a significant increase in transcutaneous $\mathrm{PCO}_{2}$ when oxygen was given at $8 \mathrm{~L}$ per minute via simple face mask. Of 106 patients randomised to high-concentration oxygen or targeted oxygen therapy (target 93-95\%), all 10 instances of hypercapnia occurred in patients given high-concentration oxygen therapy. Hypercapnia in acute asthma indicates a near-fatal attack and indicates the need for consideration of intensive care admission and ventilation. ${ }^{229}$ The trial of Perrin et al suggests that many cases of hypercapnic respiratory failure in acute asthma may be due to excessive oxygen therapy. It remains appropriate to give oxygen to patients with acute severe asthma in the absence of oximetry or blood gas results, but there is no evidence of benefit from giving oxygen to patients who are not hypoxaemic and the above study suggests that unnecessary oxygen administration may cause harm. Oxygen should not be withheld from hypoxaemic patients with severe asthma because of concerns about possible hypercapnia, but oxygen therapy should be confined to what is necessary to achieve a saturation range of $94-98 \%$. $^{266} 267$

Evidence statements

- Patients with acute asthma should not be given highconcentration oxygen therapy in the absence of severe hypoxaemia (evidence level 4).

- The suggested target saturation range of $94-98 \%$ in acute asthma is based on expert opinion in the absence of randomised trials (evidence level 4).

\section{Recommendation}

F1: In acute asthma, aim at an oxygen saturation of 94-98\% (see tables 2 and 3 and sections 8.11 and 8.13) (grade D).

\subsubsection{Pneumonia}

The BTS guideline for pneumonia recommends aiming at an oxygen saturation target range of $94-98 \%$ in uncomplicated pneumonia with appropriate adjustments for patients with COPD, guided by blood gas measurements. ${ }^{268}$ The present guideline endorses these principles. It has recently been demonstrated that high-concentration oxygen therapy can increase transcutaneous $\mathrm{CO}_{2}$ level in patients presenting with suspected community-acquired pneumonia. ${ }^{137}$

Evidence statement

- The use of oxygen in pneumonia is based on expert opinion (evidence level 4).

Recommendation

F2: In cases of pneumonia who are not at risk of hypercapnic respiratory failure, aim at an oxygen saturation of $94-98 \%$ (grade D).

\subsubsection{Lung cancer and other cancers with pulmonary} involvement

Most patients with cancer who present with acute breathlessness have a specific causative factor such as a pleural effusion, 
pneumonia, COPD, anaemia or collapse of a lobe or collapse of a whole lung. ${ }^{269} 270$ Relief of breathlessness may be achieved by treating these causative factors or by the use of opioid medicines but there is increasing evidence that supplemental oxygen does not relieve breathlessness for non-hypoxaemic patients with cancer, as discussed in section 8.17. However, it is likely that patients with cancer with significant hypoxaemia may have some relief from breathlessness if given oxygen.

Evidence statement

- The use of oxygen in the acute management of lung cancer is based on expert opinion and extrapolation from observational studies (see separate advice on palliative care in section 8.17; evidence level 4).

\section{Recommendation}

F3: In acute breathlessness due to lung cancer, aim at an oxygen saturation of $\mathbf{9 4 - 9 8 \%}$ unless there is coexisting COPD. See also 'Oxygen use in palliative care' section 8.17 (grade D).

\subsubsection{Deterioration of fibrotic lung conditions and other} conditions involving parenchymal lung disease or alveolitis It is recognised that patients with fibrosing lung conditions such as idiopathic pulmonary fibrosis may have acute deteriorations or exacerbations, often during intercurrent chest infections. Other patients may present acutely with breathlessness due to extrinsic allergic alveolitis, sarcoidosis or other types of parenchymal lung disorders. These patients often have a high degree of V/Q mismatch and a requirement for high oxygen concentrations to achieve satisfactory blood gases and they are not at risk of hypercapnia. The oxygen level should be adjusted to maintain an oxygen saturation in the range of 94-98\%, but this level may not be achievable or only achievable with a reservoir mask. Patients with severe idiopathic pulmonary fibrosis are rarely suitable for invasive ventilation or NIV because of the progressive nature of the condition.

Evidence statement

- The use of oxygen in deteriorating pulmonary fibrosis is based on expert opinion (evidence level 4).

Recommendation

F4: In acute deterioration of pulmonary fibrosis or other interstitial lung diseases, aim at an oxygen saturation of 94$98 \%$ or the highest possible if these targets cannot be achieved (grade D).

\subsubsection{Pneumothorax}

As with pleural effusions, patients with a large pneumothorax may be breathless and hypoxaemic and may require supplemental oxygen for symptom relief pending definitive treatment by aspiration or drainage. However, high-concentration inhaled oxygen can also increase the rate of reabsorption of air from a pneumothorax up to fourfold. ${ }^{271}$ A 2012 study using rabbits with traumatic pneumothorax confirmed that resolution time was significantly faster with high $\mathrm{FiO}_{2}$ but there was evidence of lung toxicity above $60 \%$ oxygen. ${ }^{272}$ The BTS guideline on the management of pneumothorax recommends the use of highconcentration oxygen (reservoir mask) in all patients without COPD who require hospital admission for observation due to a moderate-sized pneumothorax that does not require drainage. ${ }^{273}$ Once a pneumothorax is drained or aspirated successfully, the patient should not require oxygen therapy unless there is additional pathology such as pneumonia, asthma or COPD requiring specific treatment.

\section{Evidence statement}

- The use of oxygen in pneumothorax is based on expert opinion and extrapolation from observational studies (evidence level 4).

\section{Recommendations}

F5: In most cases of pneumothorax, aim at an oxyen saturation of $94-98 \%$ if the patient is not at risk of hypercapnic respiratory failure (grade $\mathrm{D}$ ).

F6: In patients with pneumothorax having hospital observation without drainage, the use of high-concentration oxygen (15 $\mathrm{L} / \mathrm{min}$ flow rate via reservoir mask) is recommended unless the patient is at risk of hypercapnic respiratory failure (grade $\mathrm{D})$.

\subsubsection{Pleural effusion}

If a pleural effusion is causing significant breathlessness, the most effective treatment is to drain the effusion. Hypoxaemic patients with pleural effusions are likely to benefit from supplementary oxygen therapy. The BTS guidelines for management of pleural effusions do not give any specific advice concerning oxygen therapy, but it seems reasonable to give supplementary oxygen to hypoxaemic patients to maintain a saturation of 94 $98 \%$.

\section{Evidence statement}

- The use of oxygen in pleural effusion is based on expert opinion (evidence level 4).

Recommendation

F7: In pleural effusion, aim at an oxygen saturation of 94$98 \%$ (or $88-92 \%$ if the patient is at risk of hypercapnic respiratory failure) (grade D).

\subsubsection{Pulmonary embolism}

Most patients with suspected pulmonary embolism have normal oxygen saturation and the main focus of treatment is to reach a specific diagnosis and to start anticoagulant treatment. These patients do not require oxygen therapy unless there is hypoxaemia. In these cases, the lowest concentration of oxygen that will achieve a target saturation of $94-98 \%$ is recommended. However, patients with massive or multiple pulmonary embolism may be profoundly hypoxaemic and should initially be given high-concentration oxygen via a reservoir mask to achieve an oxygen saturation of $94-98 \%$ pending definitive treatment such as thrombolysis. It has been suggested that the blood oxygen saturation may underestimate the severity of pulmonary artery obstruction in acute pulmonary embolism if shock is present. $^{274}$

Evidence statement

- The use of oxygen in pulmonary embolism is based on expert opinion (evidence level 4).

Recommendation

F8: In pulmonary embolism, aim at an oxygen saturation of $94-98 \%$ (or $88-92 \%$ if the patient is at risk of hypercapnic respiratory failure) (grade $\mathrm{D}$ ).

\subsubsection{Acute heart failure}

Most patients with acute heart failure are breathless, usually due to pulmonary oedema or low cardiac output, especially if cardiogenic shock is present. The pathophysiology of oxygen transport in cardiogenic shock has been discussed in detail by Creamer et al. ${ }^{275}$ It has been shown in an animal model that the ventilatory failure of cardiogenic shock may be due to an impairment of the contractile process of the respiratory muscles. ${ }^{276}$ Miñana et $a l^{277}$ did not find increased mortality in Spanish patients with acute heart failure who had hypoxaemia defined as $\mathrm{PaO}_{2}<60 \mathrm{~mm} \mathrm{Hg}(8 \mathrm{kPa})$ which would correspond to saturation below about $91 \%$ at normal $\mathrm{pH}$. In addition to specific treatment for heart failure, patients should be given supplemental oxygen to maintain a saturation of $94-98 \%$. This is 
consistent with the European Society of Cardiology Task Force and European Society of Intensive Care recommendation that patients with acute heart failure should receive oxygen to maintain $\mathrm{SpO}_{2}$ of $>90 \% .^{278}$ Patients with marked hypoxaemia (saturation $<85 \%$ ) should be treated with a reservoir mask initially and patients with coexisting COPD will require a lower target saturation of $88-92 \%$ pending the availability of blood gas results.

\section{Evidence statements}

- The use of oxygen in acute heart failure is based on expert and extrapolation from observational studies (evidence level 4).

- In hospital settings, patients with acute pulmonary oedema may benefit from CPAP and from non-invasive ventilatory support (evidence level $1+$, see evidence statement in section 8.19.2).

\section{Recommendations}

F9: In acute heart failure, aim at an oxygen saturation of 94$98 \%$ (or $88-92 \%$ if the patient is at risk of hypercapnic respiratory failure) (grade D).

F10: CPAP with entrained oxygen or high-flow humidified nasal oxygen to maintain saturation $94-98 \%$ (or $88-92 \%$ if at risk of hypercapnia) should be considered as an adjunctive treatment to improve gas exchange in patients with cardiogenic pulmonary oedema who are not responding to standard treatment (or NIV if there is coexistent hypercapnia and acidosis) (grade B).

\subsubsection{Breathlessness due to severe anaemia}

- If breathlessness is due to severe anaemia, the specific treatment is blood transfusion. Studies by Canadian researchers in the late 1990s have shown that haemoglobin levels of $70 \mathrm{~g} / \mathrm{L}(7 \mathrm{~g} / \mathrm{dL})$ were as safe as transfusion to higher levels and may produce fewer complications in the critically ill. ${ }^{72}$ However, this study was conducted using non-leucocyte-depleted blood and it is possible that some of the complications in the group who were given more transfusions might have been avoided by the use of leucocytedepleted blood. The optimal transfusion target for critically ill patients therefore remains the subject of ongoing discussion among experts in critical care medicine and readers are referred to national guidelines for blood transfusion. ${ }^{279}$ Giving oxygen to increase an already normal oxygen saturation will have very little effect on the oxygen-carrying power of the blood, but it is reasonable to administer supplemental oxygen to maintain a saturation of $94-98 \%$ if the saturation is below these levels breathing air or if breathlessness is a very prominent symptom.

Evidence statement

- The use of oxygen in anaemia is based on expert opinion based on blood gas physiology (evidence level 4).

\section{Recommendations}

F11: In anaemia, aim at an oxygen saturation of $94-98 \%$ or $88-92 \%$ if the patient is at risk of hypercapnic respiratory failure (grade D).

Good practice point

$\checkmark$ Correction of anaemia by blood transfusion should be based on national guidelines.

\subsubsection{Sickle cell crisis}

Patients with sickle cell disease frequently present with an acute painful crisis and less frequently with an 'acute chest syndrome' comprising breathlessness, chest pain and fever with pulmonary infiltrates on the chest radiograph. The exact causes and mechanisms are not well understood, but oxygen should be given to all hypoxaemic patients with sickle cell crisis to avoid further intravascular sickling. There are no randomised studies of oxygen therapy in acute chest syndrome and no randomised studies of acute painful crisis in adults, but two small randomised trials showed no clinical benefit in non-hypoxaemic children with acute painful crisis. ${ }^{280} 281$ Patients with sickle cell disease may have a reduced oxygen saturation even when clinically stable. Homi et $a l^{282}$ reported a mean saturation of only $92.5 \%$ (95\% CI $92.0 \%$ to $93.0 \%)$ in a group of children and young adults (age 9-18 years) with stable sickle cell disease compared with an average saturation of $97.1 \%$ (95\% CI $98.8 \%$ to $97.3 \%)$ in a local control group. The British Committee for Standards in Haematology recommended in 2003 that oxygen should be given if the oxygen saturation falls below what is normal for the individual patient or a default target of $95 \%$ if the usual saturation is unknown. ${ }^{283}$ The 2012 NICE guideline reinforced this advice. ${ }^{284}$ This is consistent with the advice in the present guideline to aim at a normal or near-normal oxygen saturation for non-hypoxaemic patients with a target saturation of $94-98 \%$. Readers are referred to the guideline on sickle cell disease for disease-specific management of this condition. ${ }^{283}$

Evidence statement

- During an acute sickle cell crisis, low $\mathrm{SaO}_{2}$ as assessed by pulse oximetry is common. Research suggests that this usually indicates a low $\mathrm{PaO}_{2}$ although during an acute painful crisis pulse oximetry may underestimate the true $\mathrm{SaO}_{2}$. Desaturations during an acute sickle cell crisis should be treated with oxygen to reduce further sickling and progression of the crisis with further vaso-occlusive events (evidence level 1-).

Recommendation

F12: In sickle cell crisis and acute chest syndrome, aim for an oxygen saturation of $94-98 \%$ or aim at the saturation level that is usual for the individual patient (grade $\mathrm{D}$ ).

Good practice point regarding sickle cell crisis

$\checkmark$ Arterial or arterialised capillary blood gases should be sampled if there is any doubt about the reliability of oximetry during a sickle cell crisis.

\subsection{Recommended oxygen therapy for patients who may} be vulnerable to medium or high concentration of oxygen Tables 1-4 are based on section 8, they are shown at the front of the document (see also table 3 ).

COPD is the best known condition that can predispose to hypercapnic (type 2) respiratory failure with acidosis, especially if the blood oxygen level is increased above $10 \mathrm{kPa}(75 \mathrm{~mm}$ $\mathrm{Hg}){ }^{43}{ }^{45}$ However, there are a number of other conditions which can render patients vulnerable to hypercapnic respiratory failure. The emphasis for such patients is to avoid clinically harmful levels of hypoxaemia or hypercapnia by giving carefully titrated oxygen therapy or, if necessary, by supporting the patient with the use of non-invasive or invasive mechanical ventilation.

Patients without COPD at risk of hypercapnic respiratory failure include the following:

- CF.

- Non-CF bronchiectasis (often in association with COPD or severe asthma).

- Severe kyphoscoliosis or severe ankylosing spondylitis.

- Severe lung scarring from old tuberculosis (especially with thoracoplasty).

- Morbid obesity (BMI $\left.>40 \mathrm{~kg} / \mathrm{m}^{2}\right)$.

- Musculoskeletal disorders with respiratory muscle weakness, especially if on home ventilation.

- Overdose of opioids, benzodiazepines or other respiratory depressant drugs. 


\subsubsection{COPD exacerbations}

There is an extensive literature documenting the effects of highconcentration oxygen therapy in acute COPD. ${ }^{43-}$

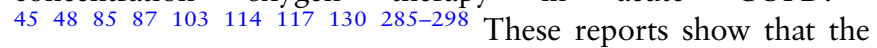
administration of supplemental oxygen to patients with exacerbated COPD (or with severe but stable COPD) often causes a rise in $\mathrm{PaCO}_{2}$ with subsequent respiratory acidosis for reasons summarised in section 5.3. The literature up to 2001 is summarised in detail in the review by Murphy et al. ${ }^{84}$ Some patients with COPD are prone to repeated episodes of hypercapnic respiratory failure in response to oxygen therapy and others may not ever suffer from this complication. Apart from patients with recurrent hypercapnic respiratory failure, it is not possible to predict if individual patients with COPD will develop hypercapnia during an acute exacerbation, so all patients with moderate or severe COPD should be considered to be at risk of this complication until the results of blood gas measurements are available. It is therefore important that patients who are at risk of having COPD should be diagnosed accurately, and this can only be done by measurement of spirometry. ${ }^{1}$ The advice given for patients with COPD also applies to other forms of fixed airflow obstruction such as patients with chronic irreversible asthma or fixed airflow obstruction associated with bronchiectasis.

Some patients with acute severe exacerbations of COPD may be too breathless to undertake spirometry on arrival in hospital, but NICE guidance is that all patients should have the test performed before discharge from hospital to confirm the diagnosis of COPD and to assess the severity of the condition. ${ }^{1}$ There is very little literature describing the effects of oxygen therapy in the other conditions listed above, but patients with these conditions are recognised to be at risk of hypercapnic respiratory failure and should be treated in a manner analogous to patients with COPD.

It has been shown that patients with COPD with a $\mathrm{pH}$ reading $<7.35\left(\left[\mathrm{H}^{+}\right]>45 \mathrm{nmol} / \mathrm{L}\right)$ despite controlled oxygen therapy are more likely to die and more likely to meet criteria for intubation and ventilation. ${ }^{43285} 289$ One of these reports also showed that patients with a high $\mathrm{PaO}_{2}$ on arrival in hospital (>10.0 kPa or $75 \mathrm{~mm} \mathrm{Hg}$ ) were more likely to meet criteria for ventilation and the severity of acidosis was related to high $\mathrm{PaO}_{2}$ values. $^{43}$ Based on these results, Plant et $a l^{43}$ recommended an upper limit of about $92 \%$ saturation for patients with exacerbations of COPD to prevent the $\mathrm{PaO}_{2}$ rising above $10 \mathrm{kPa}$. This report was supported by the work of Joosten et $a l^{45}$ which showed that $\mathrm{a} \mathrm{PaO}_{2}$ of $>74.5 \mathrm{~mm} \mathrm{Hg}(10 \mathrm{kPa})$ in acute COPD was associated with an increased likelihood of admission to a HDU, increased need for NIV and a longer stay in hospital.

Roberts et $a l^{46}$ reported outcomes among 5052 patients with exacerbated COPD where oxygen therapy in the ambulance was documented. They found that the mortality was $7.2 \%$ in patients who were managed with $<35 \%$ oxygen but mortality was $11.1 \%$ in those given $\geq 35 \%$ oxygen and the need for NIV increased from $9 \%$ to $22 \%$ when high-flow oxygen was given.

Based on the above literature, this guideline recommends a maximum saturation of $92 \%$ while awaiting blood gas results in AECOPD and other conditions that may predispose to hypercapnic respiratory failure. Although the rise in $\mathrm{PaCO}_{2}$ (and fall in $\mathrm{pH}$ ) is greatest in patients who are given sufficient oxygen therapy to elevate the $\mathrm{PaO}_{2}$ above $10 \mathrm{kPa}$, it is important to note that hypercapnia can occur in acute COPD even if the oxygen saturation is $<88 \%$. $^{296}$ The best management strategy for patients with persistently acidotic COPD is a trial of NIV with supplemental oxygen therapy, if required to maintain oxygen saturations 88-92\%. ${ }^{43209} 299$
Some patients with previous hypercapnic respiratory failure will have alert cards or an entry in their electronic record to alert the emergency team to the optimal concentration of oxygen required during the patient's previous hospital admissions (see section 9.7). In the absence of such information, it is suggested that a target of $88-92 \%$ should be set initially for patients with a history of previous NIV or invasive ventilation and, if necessary, modified later based on blood gas results. These patients should be categorised as very urgent by ambulance teams and emergency services, requiring immediate blood gas measurement and senior assessment on arrival at the hospital emergency department. Against this background, the study of Austin et $a l^{51}$ has strengthened the advice given in this guideline. These authors randomised 405 patients with presumed exacerbation of COPD to high-concentration oxygen (8-10 L/ min via reservoir mask) or to titrated oxygen treatment with target range 88-92\% using nasal cannulae (with nebulised bronchodilator therapy driven by compressed air). Mortality was $9 \%$ among those given high-concentration oxygen and 4\% among those randomised to targeted oxygen therapy. For patients with spirometrically confirmed COPD, mortality was $9 \%$ vs $2 \%$, respectively. Further support for targeted oxygen therapy was supplied in the 2012 study of Cameron et al. ${ }^{135}$ They undertook retrospective review of the records of 254 COPD admissions to one hospital and they found that both hypoxaemia $\left(\mathrm{PaO}_{2}<60 \mathrm{~mm} \mathrm{Hg}\right.$ or $\left.6 \mathrm{kPa}\right)$ and hyperoxaemia $\left(\mathrm{PaO}_{2}>100 \mathrm{~mm} \mathrm{Hg}\right.$ or $\left.13.3 \mathrm{kPa}\right)$ were associated with increased risk of serious adverse outcome, defined as a composite of hypercapnic respiratory failure, assisted ventilation or inpatient death. Twenty-four per cent of patients were hyperoxaemic and these patients had OR of 9.2 for serious adverse outcome compared with normoxaemia. Hypoxaemia occurred in 33\% of patients and was associated with an OR of 2.2 for serious adverse outcome. Expressed in terms of the BTS guideline target range, the OR for serious adverse outcome was 2.0 for those with saturation below 88\% compared with 88-92\%. There was no increase in risk in the range of $93-96 \%$ but the OR for serious adverse outcome was 2.4 if the saturation was above $96 \%$. The evidence for targeted oxygen therapy in exacerbated COPD is now so strong that it is unlikely that ethical approval would be granted for any further trials that randomised patients to high-concentration oxygen. However, it is not known if $88-92 \%$ is necessarily the ideal saturation range and it would be ethical to randomise patients with COPD to targeted oxygen with a target range slightly above or below or slightly wider or narrower than $88-92 \%$.

Unfortunately, clinical studies have shown that patients with COPD are frequently given very high concentrations of oxygen, either because of misdiagnosis or because the risks of hyperoxia in patients with COPD have been overlooked. ${ }^{43-45} 285$ Many patients with COPD are unaware of the diagnosis or are mislabelled as having asthma (see section 9.5). The diagnosis of COPD should be communicated clearly to the patient because ambulance teams have difficulty differentiating asthma and COPD in the prehospital setting. In a recent Australian study, only $57 \%$ of 1048 patients recorded as COPD by ambulance teams had an emergency department discharge diagnosis of COPD. ${ }^{300}$

The consensus from the literature in the past was that patients with AECOPD should be treated with Venturi masks to minimise the risks of hypercapnic respiratory failure. ${ }^{84}$ However, with the availability of continuous oximetry during medical emergencies, it is now possible to titrate the flow of oxygen from nasal cannulae to achieve the desired target range and nasal cannulae 
were used in the only randomised trial of targeted oxygen therapy for patients with COPD. ${ }^{51}$ If Venturi masks are used, it is not yet known if it is better to start with a $28 \%$ Venturi mask or a $24 \%$ Venturi mask. Initial management with a $28 \%$ Venturi mask appears to be safe. ${ }^{301}$ The current guideline recommends starting with either a $28 \%$ Venturi mask of $1-2 \mathrm{~L}$ of nasal oxygen in cases of COPD with no known history of hypercapnic respiratory failure, with downward adjustment to a $24 \%$ mask (in hospital) if the saturation rises above $92 \%$. In cases of prior hypercapnic failure who do not have an oxygen alert card, it is recommended that prehospital treatment should be started using a $28 \%$ Venturi mask at $4 \mathrm{~L} / \mathrm{min}$ or a $24 \%$ Venturi mask in hospitals (and prehospital if available) with a target saturation of 88$92 \%$. Observational studies in the 1960s suggested that a $\mathrm{PaO}_{2}$ of $50 \mathrm{~mm} \mathrm{Hg}$ or $6.7 \mathrm{kPa}$ (saturation about $84 \%$ ) will prevent death from hypoxaemia in acute COPD exacerbations. ${ }^{290} 291$ If the saturation should fall below $88 \%$ despite treatment with a $24 \%$ or $28 \%$ Venturi mask, the patient should be treated with nasal cannulae or a simple face mask with the flow adjusted to maintain a saturation of $88-92 \%$ pending the availability of blood gas results. This small subgroup of patients is at very high risk of death and should be treated as a high priority on arrival in emergency departments, requiring immediate senior assessment and ABG measurements.

Evidence statements

- The target saturation in patients with COPD who are at risk of hypercapnia is $88-92 \%$ (evidence level $1+$ ).

- The target saturation for patients with other risk factors for hypercapnia (eg, morbid obesity, chest wall deformities or neuromuscular disorders or fixed airflow obstruction associated with bronchiectasis) is $88-92 \%$ based on expert opinion which is extrapolated from observational studies (evidence level 4).

Recommendations

G1 (also A3): For most patients with known COPD or other known risk factors for hypercapnic respiratory failure (eg, morbid obesity, CF, chest wall deformities or neuromuscular disorders or fixed airflow obstruction associated with bronchiectasis), a target saturation range of $88-92 \%$ is suggested pending the availability of blood gas results (grade A for COPD, grade $\mathrm{D}$ for other conditions).

G2: Some patients with COPD and other conditions are vulnerable to repeated episodes of hypercapnic respiratory failure. In these cases, it is recommended that treatment should be based on the results of previous blood gas estimations during acute exacerbations. For patients with prior hypercapnic failure (requiring NIV or intermittent positive pressure ventilation) who do not have an alert card, it is recommended that low-concentration oxygen treatment should be started using a $24 \%$ Venturi mask at $2-3 \mathrm{~L} / \mathrm{min}$ (or a $28 \%$ Venturi mask at $4 \mathrm{~L} / \mathrm{min}$ or nasal cannulae at $1-2 \mathrm{~L} / \mathrm{min}$ if a $24 \%$ mask is not available) with an initial target saturation of $88-92 \%$ pending urgent blood gas results. these patients should be treated as a high priority by emergency services and the oxygen concentration should be reduced if the saturation exceeds $92 \%$ but increased if it falls below $88 \%$ (grade D).

Good practice points for COPD and other conditions that may cause hypercapnic respiratory failure

Diagnosis of COPD or suspected exacerbation of COPD:

$\checkmark$ If the diagnosis is unknown, patients over 50 years of age who are long-term smokers with a history of chronic breathlessness on minor exertion such as walking on level ground and no other known cause of breathlessness should be treated as having suspected COPD for the purposes of this guideline. $\checkmark$ Spirometry should be measured at least once during hospital admissions for suspected COPD (as per NICE COPD guideline ${ }^{1}$ ). Measurement of spirometry may confirm (or exclude) a diagnosis of airflow obstruction and the $\mathrm{FEV}_{1}$ level is a useful indicator of disease severity in COPD.

Immediate management of patients with known or suspected COPD:

$\checkmark$ If the saturation remains below $88 \%$ in prehospital care despite a $28 \%$ Venturi mask, change to nasal cannulae at 2 $6 \mathrm{~L} / \mathrm{min}$ or a simple face mask at $5 \mathrm{~L} / \mathrm{min}$ with target saturation of $88-92 \%$ and alert the emergency department that the patient is to be treated as a high priority.

$\checkmark$ Patients with a respiratory rate $>30$ breaths/min should have the flow rate from Venturi masks set above the minimum flow rate specified for the Venturi mask packaging to compensate for the patient's increased inspiratory flow (see figure 11B). Increasing the oxygen flow rate into a Venturi mask does not increase the concentration of oxygen which is delivered.

$\checkmark$ Patients with a significant likelihood of severe COPD or other illness that may cause hypercapnic respiratory failure should be triaged as very urgent on arrival in hospital emergency departments and blood gases should be measured on arrival in hospital.

$\checkmark$ Prior to availability of blood gas measurements, use a $24 \%$ Venturi mask at $2-3 \mathrm{~L} / \mathrm{min}$ or nasal cannulae at $1-2 \mathrm{~L} / \mathrm{min}$ or $28 \%$ Venturi mask at $4 \mathrm{~L} / \mathrm{min}$ and aim for an oxygen saturation of $88-92 \%$

Initial hospital management of patients with exacerbation of COPD:

$\checkmark$ Patients with exacerbations of COPD need careful monitoring for hypercapnic (type 2) respiratory failure with respiratory acidosis which may develop in the course of a hospital admission even if the initial blood gases were satisfactory.

$\checkmark$ Avoid excessive oxygen use in patients with COPD. The risk of respiratory acidosis in patients with hypercapnic respiratory failure is increased if the $\mathrm{PaO}_{2}$ is above $10.0 \mathrm{kPa}$ due to previous excessive oxygen use.

$\checkmark$ If following blood gas measurements the $\mathrm{pH}$ and $\mathrm{PCO}_{2}$ are normal, aim for an oxygen saturation of $94-98 \%$ unless there is a history of previous hypercapnic respiratory failure requiring NIV or intermittent positive pressure ventilation or if the patient's usual oxygen saturation when clinically stable is below 94\% (these patients should have a target range of $88-92 \%)$. Blood gases should be repeated at 30-60 min to check for rising $\mathrm{PCO}_{2}$ or falling $\mathrm{pH}$.

$\checkmark$ Recheck blood gases after 30-60 min (or if there is evidence of clinical deterioration) for all patients with COPD or other risk factors for hypercapnic respiratory failure even if the initial $\mathrm{PCO}_{2}$ measurement was normal.

$\checkmark$ If the $\mathrm{PCO}_{2}$ is raised but $\mathrm{pH}$ is $\geq 7.35\left(\left[\mathrm{H}^{+}\right] \leq 45 \mathrm{nmol} / \mathrm{L}\right)$ and/ or a high bicarbonate level $(>28 \mathrm{mmol} / \mathrm{L})$, the patient has probably got long-standing hypercapnia; maintain target range of $88-92 \%$ for these patients. Blood gases should be repeated at 30-60 min to check for rising $\mathrm{PCO}_{2}$ or falling $\mathrm{pH}$.

$\checkmark$ If the patient is hypercapnic $\left(\mathrm{PCO}_{2}>6 \mathrm{kPa}\right.$ or $\left.45 \mathrm{~mm} \mathrm{Hg}\right)$ and acidotic ( $\mathrm{pH}<7.35$ or $\left[\mathrm{H}^{+}\right]>45 \mathrm{nmol} / \mathrm{L}$ ), start NIV with targeted oxygen therapy if respiratory acidosis persists for more than $30 \mathrm{~min}$ after initiation of standard medical management.

$\checkmark$ For patients using Venturi masks, consider changing from Venturi mask to nasal cannulae once the patient has stabilised. 
$\checkmark$ For patients who use LTOT for severe COPD, a senior clinician should consider setting a patient-specific target range if the standard range of $88-92 \%$ would require inappropriate adjustment of the patient's usual oxygen therapy while the patient is in hospital.

Management of hypercapnia or respiratory acidosis due to excessive oxygen therapy-avoidance of life-threatening rebound bypoxaemia (see section 6.3.2)

$\checkmark$ If a patient is suspected to have hypercapnic respiratory failure due to excessive oxygen therapy, the oxygen therapy must be stepped down to the lowest level required to maintain a saturation range of $88-92 \%$. This may be achieved using $28 \%$ or $24 \%$ oxygen from a Venturi mask or $1-2 \mathrm{~L} /$ min via nasal cannulae depending on oxygen saturation and subsequent blood gas measurements.

$\checkmark$ Sudden cessation of supplementary oxygen therapy can cause life-threatening rebound hypoxaemia with a rapid fall in oxygen saturations below the starting oxygen saturation prior to the start of supplementary oxygen therapy.

\subsubsection{Exacerbation of CF}

Patients with breathlessness due to CF should be managed in a Cystic Fibrosis Centre unless this is not possible for geographical reasons. If not possible, all cases should be discussed with the Cystic Fibrosis Centre or managed according to a protocol that has been agreed with the regional centre. Patients with advanced CF may suffer from exacerbations which are similar to exacerbations of advanced COPD with associated hypoxaemia and hypercapnia. The principles of management are similar to those in AECOPD, including a need to maintain adequate oxygen saturation and avoiding excessive hypercapnia and acidosis. As in COPD, NIV may be of value in severe cases. ${ }^{302}$ NIV in CF may also be helpful to reduce symptoms (eg, work of breathing and breathlessness) and assist in airway clearance.

It is recommended that patients with acute exacerbations of CF should be managed on similar lines to patients with AECOPD with a target oxygen saturation of $88-92 \%$ for most patients, but recognition that individual patients may need to be managed differently on the basis of previous and current blood gas measurements. One study has shown that patients with a respiratory rate above 30 breaths/min often have an inspiratory flow rate above the minimum flow rate specified on the mask packaging and may therefore benefit from a doubling of this flow rate. ${ }^{303}$ However, there is no direct experimental evidence of the clinical effectiveness of increased flow rates from Venturi devices. It is possible that patients with very high inspiratory flow rates might benefit from a $28 \%$ Venturi mask with the flow rate set at $6-8 \mathrm{~L} / \mathrm{min}$ to minimise the risk of the inspiratory flow rate exceeding the gas flow rate (see section 10.2.3). Patients with CF who have had previous episodes of hypercapnic respiratory failure should be issued with an oxygen alert card with recommendations based on previous blood gas measurements.

Evidence statement

- The optimal oxygen saturation level for patients with acute exacerbations of CF has not yet been investigated in clinical trials. Expert opinion advises treating these patients in a similar manner to patients with AECOPD who are at risk of hypercapnic respiratory failure (evidence level 4).

Recommendation

G3: Initial oxygen treatment of CF exacerbations should be similar to the initial oxygen treatment of COPD exacerbations with target saturation $88-92 \%$ (see sections 8.12.1-8.12.2) (grade D).
8.12.3 Chronic musculoskeletal and neurological disorders

Hypoxaemia due to musculoskeletal and neurological disorders may be associated with acute illness (such as a chest infection) superimposed on a chronic neuromuscular condition. However, muscle weakness can be acute, subacute (eg, Guillain-Barre' syndrome) or chronic (see section 8.13.6). For most patients with inadequate ventilation due to neuromuscular weakness, noninvasive or invasive ventilatory support is more useful than supplementary oxygen and these patients are at risk of hypercapnic respiratory failure which may be aggravated by high concentrations of oxygen. For this reason, it is recommended that spirometry should be monitored carefully and blood gases should be obtained as early as possible in all such cases. Pending the availability of blood gas results, a saturation target of $88-92 \%$ will avoid the risks of severe hypoxaemia or severe hypercapnia. The NICE guidance on NIV in motor neurone disease is also applicable to other neuromuscular conditions causing respiratory failure. $^{304}$

\section{- Evidence statement}

The optimal oxygen saturation level for patients with musculoskeletal and neurological disorders has not yet been investigated in clinical trials. Expert opinion advises urgent blood gas assessment and early consideration of NIV with a target saturation of $88-92 \%$ while blood gas results are awaited (evidence level 4).

Recommendation

G4: In the initial management of musculoskeletal and neurological disorders with acute respiratory failure or acute-on-chronic respiratory failure, aim at an oxygen saturation of $88-92 \%$ and measure blood gases to determine if NIV will be required (grade D).

Good practice point regarding patients with neurological disorders

$\checkmark$ Patients with respiratory failure due to neurological disorders or muscle disease are at high risk of dying and require urgent assessment to determine if they are likely to require non-invasive or invasive ventilator support rather than oxygen therapy. Monitor these patients with blood gases and regular spirometry (forced vital capacity). Patient's wishes regarding this form of treatment should be established as early as possible in the course of the illness, ideally before an acute episode has developed.

\subsubsection{Obesity-hypoventilation syndrome}

Patients with the OHS are chronically hypercapnic unless treated with NIV and may decompensate acutely resulting in hypercapnic respiratory failure with acidosis. ${ }^{305}$ One study has found the incidence of hypoventilation to be as high as $31 \%$ in a cohort of obese hospitalised patients. ${ }^{306}$ Wijesinghe et al ${ }^{47}$ have demonstrated in 2011 that breathing 100\% oxygen causes worsening hypercapnia in stable patients with obesity-associated hypoventilation. For purposes of oxygen therapy, patients with morbid obesity should be treated in a similar manner to patients with an AECOPD. The target saturation will usually be $88-92 \%$ but, as with COPD, a lower target range may be appropriate for individual patients based on blood gas measurements during a previous exacerbation or due to acute acidosis. Assessment of patients with increasing shortness of breath or worsening oxygen saturation must include blood gases. As in COPD, patients with respiratory acidosis may benefit from NIV.

Evidence statement

- Patients with OHS are at risk of worsening hypercapnia when administered high-flow oxygen (evidence level 1+). 
- However, the optimal oxygen saturation level for patients with OHS has not yet been investigated in clinical trials. Expert opinion advises treating these patients in a similar manner to patients with AECOPD who are at risk of hypercapnic respiratory failure (evidence level 4).

\section{Recommendations}

G5: Morbidly obese patients (BMI $\left.>40 \mathrm{~kg} / \mathrm{m}^{2}\right)$, even without evidence of coexistent OSA are at risk of hypoventilation and should be given titrated oxygen to maintain a target saturation of $88-92 \%$ (grade D).

G6: NIV should be considered for hypercapnic patients with COPD, CF, neuromuscular disorders or morbid obesity who are at risk of hypercapnic respiratory failure if the $\mathrm{pH}$ is $<7.35$ or $\left[\mathrm{H}^{+}\right]>45 \mathrm{nmol} / \mathrm{L}$ (grade D). See BTS/ICS Guideline for the ventilatory management of acute hypercapnic respiratory failure (ref 299).

\subsection{Common medical emergencies for which oxygen therapy is indicated only if hypoxaemia is present (see also table 4)}

There are a number of conditions such as myocardial infarction, angina and stroke for which oxygen was traditionally given to all patients in an attempt to increase oxygen delivery to the heart or brain. However, the administration of supplemental oxygen to normoxaemic patients has very little effect on blood oxygen content but may reduce myocardial and cerebral blood flow due to vasoconstriction which is a physiological response to hypoxia in most vital organs. There is no evidence of benefit from the administration of supplemental oxygen to non-hypoxaemic patients with these conditions and there is some evidence of possible harm, especially when used at high-flow rates, so it is recommended that oxygen should only be given to patients with these conditions if hypoxaemia is present, usually due to complications such as heart failure or pneumonia. There are no published trials supporting the use of oxygen to relieve breathlessness in non-hypoxaemic patients, and there is evidence from randomised studies that oxygen does not relieve breathlessness compared with air in non-hypoxaemic patients with COPD who are breathless following exertion. ${ }^{146}$

\subsubsection{Acute myocardial infarction, suspected myocardial infarction and acute coronary syndromes}

Some patients with acute myocardial infarction have heart failure and should be treated accordingly (see section 8.11.9). Most patients with suspected or confirmed myocardial infarction are not hypoxaemic and most are not breathless. In the case of non-hypoxaemic patients, it is not known if supplementary oxygen may be beneficial by increasing the amount of oxygen delivered to the hypoxaemic area of myocardium or whether it may actually cause vasoconstriction with increased systemic vascular resistance and reduced myocardial oxygen supply with worsened systolic myocardial performance..$^{307-315}$ A study of patients having coronary arteriography found that breathing $100 \%$ oxygen reduced coronary blood flow velocity by $20 \%$ and increased coronary resistance by $23 \% \cdot{ }^{316} \mathrm{~A}$ study of 16 healthy individuals using MRI found that coronary artery oxygen delivery fell by $11 \%$ due to decreased cardiac output and decreased left ventricular perfusion while breathing oxygen at $8 \mathrm{~L} / \mathrm{min}$ via a mask despite the increased blood oxygen content. ${ }^{317}$ However, Ranchord et $a l^{318}$ found that highconcentration oxygen increased the time to onset of exercise-induced myocardial ischaemia in patients with stable ischaemic heart disease, suggesting that the physiology of oxygen delivery to the heart may be different during exercise in ischaemic heart disease. Reduced myocardial oxygen consumption might also be associated with reduced contractility.

There is a theoretical possibility that high oxygen levels might exacerbate reperfusion injury to the heart. ${ }^{314}$ Despite a multitude of large studies of intervention in myocardial infarction, there had been only three published randomised studies of oxygen therapy prior to 2015. A randomised trial in 1976 did not identify any benefit from such therapy but found some evidence of potential harm. ${ }^{319}$ This trial reported a significantly greater rise in aspartate aminotransferase and heart rate which could suggest increased infarct size in the oxygen treated group. There was a difference in mortality between the two groups that did not reach statistical significance (3 deaths in 77 patients treated with air vs 9 deaths in 80 patients given oxygen at $6 \mathrm{~L} /$ min via a simple face mask for 24 hours). This study was undertaken at a time before angiographic interventions were possible for myocardial infarction.

Ranchord et $a l^{320}$ randomised 136 patients with ST-elevation myocardial infarction to $6 \mathrm{~L}$ of oxygen via simple face mask or titrated oxygen to achieve saturation of $93-96 \%$ for 6 hours after randomisation in a hospital setting. The trial was conducted between 2007 and 2009 and most patients had angiographic interventions. There was no difference in troponin $\mathrm{T}$ level between the groups and the number of deaths was too low (3) to permit meaningful analysis. Eighty-five per cent of patients in both groups had received oxygen in the ambulance and/or emergency department for a mean of $62 \mathrm{~min}$ prior to randomisation and almost all patients received thrombolysis or percutaneous coronary intervention within 4 hours of onset of chest pain. Therefore, this study really evaluated the effect of stopping oxygen therapy prior to therapeutic intervention in a group of patients of whom most had already received oxygen for more than 1 hour.

The third study was an open-label randomised study conducted in Russia which was reported with limited methodological details. ${ }^{321}$ Twenty-eight patients received oxygen in addition to standard therapy for $30 \mathrm{~min}$ prior to myocardial revascularisation and for 3 hours after the intervention, 30 patients received oxygen for 3 hours after the intervention and 79 patients received 'standard therapy'. Details of oxygen therapy prior to randomisation were not given. This study reported one death among 58 patients randomised to oxygen therapy and no deaths among 79 patients randomised to standard therapy but the authors reported fewer complications among those randomised to oxygen.

A systematic review and a historical review of oxygen therapy in acute myocardial ischaemia have both concluded that there was no evidence to support this practice in nonhypoxaemic patients and some evidence of possible harm. ${ }^{322} 323$ Further systematic reviews and meta-analysis in 2011 and 2012 concluded that the existing studies lacked power, no clear conclusions can be drawn and further studies are needed. ${ }^{324} 325$ One study from 1969 showed that hypoxaemia did not affect the availability of oxygen for myocardial metabolism in normal participants until the oxygen saturation fell to about $50 \%$, but evidence of myocardial ischaemia was seen at saturations of $70-85 \%$ in participants with coronary artery disease. ${ }^{326}$ In these circumstances, it is advised that patients with myocardial infarction or chest pain suspicious of myocardial infarction should be given supplementary oxygen if required to maintain a saturation of $94-98 \%$.

The study by Wilson and Channer ${ }^{4}$ in 1997 showed that desaturation below $90 \%$ was common in patients with myocardial infarction within the first 24 hours of admission to a 
coronary care unit, but these authors may not have been aware that nocturnal desaturation to this level is very common in healthy individuals. ${ }^{33}$ Wilson and Channer ${ }^{4}$ did not demonstrate any correlation between hypoxaemic events and adverse cardiac events. They did, however, show that monitoring by oximetry was inadequate in UK coronary care units in the mid-1990s.

The 2010 NICE Guideline for Chest Pain of recent onset recommends setting an oxygen target saturation range of 9498\% based on the arguments discussed above and the advice given in the 2008 BTS Emergency Oxygen Guideline. ${ }^{327}$ The 2012 guidelines for acute myocardial infarction of the European Society for Cardiology recommends the following: "Oxygen (by mask or nasal prongs) should be administered to those who are breathless, hypoxic $\left(\mathrm{SaO}_{2}<95 \%\right)$, or who have heart failure."328 The 2011 American College of Cardiology/American Heart Association guideline for Management of Patients With Unstable Angina/Non-ST-Elevation Myocardial Infarction gives the following advice: ${ }^{329}$ "Supplemental oxygen should be administered to patients with UA/NSTEMI with an arterial saturation $<90 \%$, respiratory distress, or other high-risk features for hypoxemia." This is followed by the following unreferenced advice: "It is reasonable to administer supplemental oxygen to all patients with UA/NSTEMI during the first $6 \mathrm{~h}$ after presentation." The limited available evidence supports the suggestion that clinicians should aim at normal or near-normal oxygen saturation in patients with myocardial infarction, acute coronary syndrome and chest pain suspicious of coronary artery disease. A target saturation range of $94-98 \%$ will meet all of these goals, and further research of this topic should be prioritised because this is such a common medical problem and there is so little existing evidence.

Fortunately, there is one recent published study and other forthcoming studies of oxygen use for myocardial infarction in ambulances. The AVOID study in Australia was study was designed to determine whether withholding routine supplemental oxygen therapy in patients with acute ST-elevation myocardial infarction but without hypoxaemia prior to reperfusion decreases myocardial infarct size. ${ }^{330-332}$ The results have suggested that cardiac enzyme levels were higher and infarct size at 6 months was $55 \%$ bigger as determined by cardiac MR scan when oxygen was administered. Mean peak troponin was similar in the oxygen and no oxygen groups $(57.4 \mathrm{vs} 48.0 \mu \mathrm{g} / \mathrm{L}$; ratio $1.20 ; 95 \%$ CI 0.92 to 1.56 ; $\mathrm{p}=0.18$ ). There was a significant increase in mean peak creatine kinase in the oxygen group compared with the no oxygen group (1948 vs $1543 \mathrm{U} / \mathrm{L}$; means ratio $1.27 ; 95 \% \mathrm{CI} 1.04$ to $1.52 ; \mathrm{p}=0.01)$. There was an increase in the rate of recurrent myocardial infarction in the oxygen group compared with the no oxygen group $(5.5 \%$ vs $0.9 \% ; \mathrm{p}=0.006)$ and an increase in frequency of cardiac arrhythmia $(40.4 \%$ vs $31.4 \% ; \mathrm{p}=0.05)$. At 6 months, the oxygen group had an increase in myocardial infarct size on cardiac MR ( $\mathrm{n}=139 ; 20.3$ vs $13.1 \mathrm{~g} ; \mathrm{p}=0.04) .{ }^{331}$ A subsequent publication by these authors showed that there was a significant increase in troponin and creatine kinase levels which was dependent on the total dose of supplemental oxygen (litres of supplemental oxygen received) and a typical patient receiving supplemental oxygen in the first 12 hours after ST-elevation myocardial infarction would experience an $\sim 20 \%$ increase in myocardial infarct size. ${ }^{332}$

The DETO2X-AMI trial is a further randomised trial of oxygen versus air in the management of suspected myocardial infarction and was started in Sweden and will hopefully provide more useful information concerning the management of this common medical emergency. ${ }^{333}$
In the past, most emergency calls to ambulance services because of chest pain were treated with high-concentration oxygen in accordance with the Joint Royal Colleges Ambulance Liaison Committee (JRCALC) older guidance. ${ }^{334}$ However, this guidance was updated in 2009 to reflect the advice given in the 2008 BTS Emergency Oxygen Guideline with a target saturation range of $94-98 \%$ for most patients with chest pain. ${ }^{12}$ Most such patients have a final diagnosis of undifferentiated chest pain rather than acute coronary artery syndrome and most patients with undifferentiated chest pain are normoxaemic. The clinical management of a very large number of patients was therefore changed following the introduction of the 2008 BTS guideline and subsequent publications have tended to support the concept of aiming for normoxaemia in patients with suspected myocardial infarction.

\section{Evidence statements}

- The optimal oxygen saturation level for patients with myocardial infarction and acute coronary syndromes has not yet been investigated in clinical trials. Expert opinion advises maintaining normal or near-normal saturation unless the patient is at risk of hypercapnic respiratory failure (evidence level 4).

- There is evidence from two randomised studies that the administration of oxygen to non-hypoxaemic patients in the immediate management of myocardial infarction may be associated with increased infarct size (evidence level $1+$ ).

\section{Recommendation}

F13: In myocardial infarction and acute coronary syndromes, aim at an oxygen saturation of $94-98 \%$ or $88-92 \%$ if the patient is at risk of hypercapnic respiratory failure (grade D).

\subsubsection{Stroke}

In the past, it was customary to give supplemental oxygen to all patients with stroke to try to improve cerebral oxygenation. There are four published randomised trials of oxygen therapy in patients with stroke with normal oxygen saturation, none of which have shown benefits. A study of supplemental oxygen (2 or $3 \mathrm{~L} / \mathrm{min}$ dependent on baseline oxygen saturation) for 24 hours $(n=550)$ found no difference in 1-year survival for the entire cohort of patients with stroke and no difference in survival for patients with more severe strokes. However, a post hoc subgroup analysis found that for patients with minor strokes, 1-year mortality was $18 \%$ in the group given oxygen and $9 \%$ in the group given air (OR $0.45 ; 95 \%$ CI 0.23 to 0.90 ; $\mathrm{p}=0.02) .{ }^{335} \mathrm{~A}$ pilot study of oxygen supplementation at a rate of 2 or $3 \mathrm{~L} / \mathrm{min}$, dependent on baseline oxygen saturation for 72 hours in the UK $(n=300)$ suggested better neurological recovery at 1 week, but showed no difference in survival and recovery at 6 months. ${ }^{336} 337$ The study did not find an excess risk for minor strokes in patients given oxygen. A much larger study $(n=8003)$ using the same dose and duration of oxygen treatment has just closed and is only available in abstract format so far, but confirms that routine oxygen treatment does not reduce mortality or improve recovery. ${ }^{338}$

A small randomised trial of high-concentration oxygen therapy $(10 \mathrm{~L} / \mathrm{min})$ in non-hypoxaemic patients $(n=40)$ showed no clinical benefit ${ }^{339}$ and a study of very high-flow oxygen (45 L/min) was terminated in 2009 after enrolling 85 patients because of excess mortality in the hyperoxaemia group (40\% vs $17 \%)$, the full report had not yet been published when this guideline was prepared. ${ }^{340}$ Finally, an observational study reported increased mortality associated with hyperoxaemia in ventilated patients with stroke on ICUs. ${ }^{341}$

Post-stroke hypoxaemia is common and associated with worse outcome in some small studies. ${ }^{342} 343$ The risk of hypoxaemia is 
particularly high at night, during interward transfers and in the CT scanner. ${ }^{342} 344$ Regular monitoring of oxygen saturation and treatment of all episodes of hypoxaemia has been shown to improve outcome in an observational study. ${ }^{343}$ It is therefore recommended to monitor oxygen saturation regularly (at least every 4 hours, including the night) during the acute phase of stroke and to treat every episode of hypoxaemia.

The most common causes of hypoxaemia after stroke are obstruction of airflow by secretions in the upper airway or complications of the stroke such as pneumonia, pulmonary embolism or heart failure. These must be addressed in every patient who develops hypoxaemia post-stroke.

It is recommended that patients with stroke should receive supplemental oxygen only after the airway has been cleared and if this treatment is required to achieve an oxygen saturation of 94-98\% (88-92\% for patients with coexisting risk of COPD or other risk of respiratory acidosis). High concentrations of oxygen should be avoided unless required to correct hypoxaemia. As patients with stroke tolerate nasal cannulae better than oxygen masks, oxygen should be given via nasal cannulae unless a strictly controlled oxygen concentration is indicated. 345346

There has also been some discussion concerning the optimal body position for the management of patients with stroke and potential hypoxaemia. A systematic review of clinical studies in conscious patients concluded that there was limited evidence that sitting in a chair had a beneficial effect and lying positions had a deleterious effect on oxygen saturation in patients with acute stroke with respiratory comorbidities, but patients with acute stroke without respiratory comorbidities can adopt any body position. ${ }^{161}$ The authors of this review recommended that people with acute stroke and respiratory comorbidities should be positioned as upright as possible as discussed in section 6 and recommendation A5. No trials have expressly addressed the best position in unconscious and semiconscious patients with stroke. As in other patient groups with a reduced level of consciousness the recovery position is recommended to safeguard the airway.

Evidence statements

- High concentrations of oxygen in normoxaemic patients with stroke may be associated with increased mortality (evidence level 1-).

- Routine oxygen for non-hypoxic patients with stroke does not improve recovery or reduce disability (evidence level 1-).

- Patient positioning has not been shown to affect $\mathrm{SaO}_{2}$ as measured by pulse oximetry in patients without cardiorespiratory comorbidities, but sitting out in a chair improves oxygenation in patients with pre-existing cardiorespiratory disease (evidence level 1-).

\section{Recommendation}

F14: High concentrations of oxygen should be avoided in patients with stroke, unless required to maintain normal oxygen saturation. Aim at an oxygen saturation of $94-98 \%$ or $88-92 \%$ if the patient is at risk of hypercapnic respiratory failure (grade D). Good practice points regarding stroke management

$\checkmark$ Oxygen saturation should be monitored at least every 4 hours throughout the day and night in patients with acute stroke and all episodes of hypoxaemia treated.

$\checkmark$ Patients with hypoxaemia post-stroke require medical review to establish and treat the cause.

$\checkmark$ Oxygen should only be given once the airway has been cleared and at the lowest concentration necessary to achieve an oxygen saturation of $94-98 \%$ or $88-92 \%$ if the patient is at risk of hypercapnic respiratory failure. $\checkmark$ Oxygen should be given via nasal cannulae, unless there are clear indications for a different oxygen delivery system.

$\checkmark$ Patients with stroke and cardiorespiratory comorbidities should be positioned as upright as possible, in a chair if possible (see recommendation A5).

$\checkmark$ Patients with a reduced level of consciousness after stroke should be nursed in the recovery position with the paralysed side lowest.

\subsubsection{Anxiety and hyperventilation or dysfunctional breathing}

Many patients who present to hospital with breathlessness are found to have no cardiopulmonary problems and many such patients have a specific diagnosis of hyperventilation, dysfunctional breathing, vocal cord dysfunction or panic attacks, sometimes in addition to asthma or some other underlying respiratory disorder. ${ }^{347}$ Many such patients will have an abnormally high oxygen saturation of $99 \%$ or $100 \%$ and clearly do not require supplemental oxygen therapy but some patients with vocal cord dysfunction may develop respiratory failure due to upper airway obstruction. Many other non-hypoxaemic patients will present to hospital with acute breathlessness of unknown cause, and the majority of patients with an elevated respiratory rate are likely to have an organic illness. In some cases, simple investigations will reveal a specific diagnosis such as pneumothorax or pneumonia or pulmonary embolism, but many cases remain undiagnosed. A policy of giving supplemental oxygen if the saturation falls below 94\% will avoid exposing patients with undiagnosed medical illnesses to the risk of hypoxaemia while avoiding the unnecessary use of oxygen in patients with behavioural or dysfunctional breathlessness.

Studies in normal volunteers have demonstrated that compensatory desaturation may occur shortly after voluntary hyperventilation. ${ }^{348}$ The mean $\mathrm{PaO}_{2}$ of 10 male volunteers increased from $13.7 \mathrm{kPa}(103 \mathrm{~mm} \mathrm{Hg})$ to $18.6 \mathrm{kPa}(140 \mathrm{~mm} \mathrm{Hg})$ during hyperventilation but fell to a nadir of $7.8 \mathrm{kPa}(58 \mathrm{~mm} \mathrm{Hg})$ about $7 \mathrm{~min}$ after cessation of hyperventilation and did not normalise until after a total of $17 \mathrm{~min}$ of observation. It is not known whether or not this occurs after pathological hyperventilation, but this phenomenon could cause considerable confusion if it should occur in an emergency department.

A traditional treatment for hyperventilation was to ask the participant to rebreathe from a paper bag to allow the carbon dioxide level in the blood to normalise. However, it has been shown that this practice can cause hypoxaemia with potentially fatal consequences. ${ }^{349}$ The average fall in $\mathrm{PO}_{2}$ during rebreathing was $26 \mathrm{~mm} \mathrm{Hg}(3.5 \mathrm{kPa})$ and the maximum fall was $42 \mathrm{~mm} \mathrm{Hg}$ (5.6 kPa). This guideline does not recommend rebreathing from a paper bag in cases of hyperventilation.

Good practice points regarding patients with suspected hyperventilation

$\checkmark$ Organic illness must be excluded before making a diagnosis of hyperventilation.

$\checkmark$ Patients with a definite diagnosis of hyperventilation should have their oxygen saturation monitored. Those with normal or high $\mathrm{SpO}_{2}$ do not require oxygen therapy.

$\checkmark$ Rebreathing from a paper bag can be dangerous and is NOT advised as a treatment for hyperventilation.

\subsubsection{Poisoning with substances other than carbon monoxide or cyanide}

Many poisons and drugs can cause respiratory or cardiac depression or direct toxic effects on the lungs. The treatment of individual toxic agents is beyond the scope of this guideline. Specific antidotes such as naloxone for opioids or flumazenil for 
benzodiazepines should be given if available and oxygen saturation should be monitored closely. Supplemental oxygen should be given to achieve a target saturation of $94-98 \%$ pending the results of blood gas analysis (88-92\% if at risk of hypercapnic respiratory failure, including patients with overdoses of narcotic agents). All potentially serious cases of poisoning should be monitored in a level 2 or 3 environment (HDU or ICU).

Three specific types of lung injury deserve special mention. Oxygen is known to be hazardous to patients with paraquat poisoning, ${ }^{125} 126$ and oxygen potentiates bleomycin lung injury. Because of these risks, oxygen should be given to patients with these conditions only if the oxygen saturation falls below $85 \%$. Some authors have suggested the use of hypoxic ventilation with $14 \%$ oxygen as a specific treatment for paraquat poisoning. ${ }^{350}$ Bleomycin lung injury can be potentiated by highconcentration oxygen therapy, even if given several years after the initial lung injury. ${ }^{127}$ It is therefore recommended that high concentrations of oxygen should be avoided in patients with possible bleomycin-induced lung injury and a lower oxygen saturation target range should be accepted (eg, 88-92\%).

There is evidence from animal experiments that oxygen may potentiate lung injury from aspiration of acids. ${ }^{128} 129$ The effect in humans is not known so patients with acid inhalation should have the usual adult target saturation range of 94-98\%, but it would appear prudent to aim in the lower half of the target range for these patients and clinical trials in humans are clearly required.

\section{Evidence statement}

- For most types of poisoning, the optimal oxygen saturation level has not yet been investigated in clinical trials. Expert opinion advises maintaining normal or near-normal saturation unless the patient is at risk of hypercapnic respiratory failure or unless they require high oxygen levels (carbon monoxide or cyanide poisoning) or have ingested a poison such as paraquat or bleomycin where supplemental oxygen may aggravate tissue damage (evidence level 4).

\section{Recommendations}

F15: In most poisonings, aim at an oxygen saturation of 94$98 \%$ unless the patient is at risk of hypercapnic respiratory failure (grade D).

F16: In poisoning by paraquat and poisoning by bleomycin, give oxygen only if the saturation falls below $85 \%$ and reduce or stop oxygen therapy if the saturation rises above $88 \%$ (grade D).

\subsubsection{Metabolic, endocrine and renal disorders}

Many metabolic and renal disorders can cause metabolic acidosis which increases respiratory drive as the body tries to correct the acidosis by increased excretion of carbon dioxide via the lungs. Although these patients have tachypnoea, they do not usually complain of breathlessness and most have high oxygen saturation (unless there is a coexisting pulmonary or cardiac problem). Supplementary oxygen is not required for such patients unless the oxygen saturation is reduced. In such cases, oxygen should be given to maintain a saturation of $94-98 \%$.

\section{Evidence statement}

- The optimal oxygen saturation level for patients with most metabolic, endocrine and renal disorders has not yet been investigated in clinical trials. Expert opinion advises maintaining normal or near-normal saturation unless the patient is at risk of hypercapnic respiratory failure (evidence level 4).

\section{Recommendation}

F17: In most metabolic and renal disorders, aim at an oxygen saturation of $94-98 \%$ unless the patient is at risk of hypercapnic respiratory failure (grade D).
8.13.6 Acute and subacute neuromuscular disorders producing respiratory muscle weakness

Patients with acute or subacute conditions affecting the respiratory muscles (often superimposed on chronic conditions) are at risk of sudden onset of respiratory failure with hypoxaemia and hypercapnia and may require non-invasive or invasive ventilatory support. This applies especially to patients with Guillain-Barre' syndrome for whom spirometry (forced vital capacity) should be monitored carefully as this should detect the onset of severe respiratory failure prior to the development of hypoxaemia. If the oxygen level falls below the target saturation, urgent blood gas measurements should be undertaken and the patient is likely to need ventilatory support.

Good practice point

$\checkmark$ Patients with respiratory failure due to neurological disorders or muscle disease are at high risk of dying and require urgent assessment to determine if they are likely to require non-invasive or invasive ventilator support rather than oxygen therapy. Monitor these patients with blood gases and regular spirometry (forced vital capacity). Patient's wishes regarding this form of treatment should be established as early as possible in the course of the illness, ideally before an acute episode has developed.

\subsubsection{Cluster headache}

Relief from cluster headache has been reported in $56-85 \%$ of cases. $^{351-354}$ Benefit has recently been reported for other types of headache. ${ }^{355}$ Although this could be considered as a form of emergency oxygen therapy, these patients are not breathless or hypoxaemic. Cluster headache is one of the most severe pain disorders known to humans. Patients can attend emergency departments in an acute cluster attack but because of the relatively short duration and frequency of the episodes, home treatment of the acute cluster attack is most often undertaken. For patients with cluster headache arrangements for home and ambulatory oxygen is recommended for treatment of the acute cluster headache bout. Oxygen at a flow rate of at least $12 \mathrm{~L} /$ min from a reservoir mask is recommended. ${ }^{353}$ Patients who have episodic cluster headache may not need oxygen on a longer term basis and once they have been cluster headache free for over 4 weeks the oxygen supply can be uplifted. However, because of the recurrent nature of the condition, emergency supply of oxygen should be made available for cluster headache sufferers. In a study comparing the use of oxygen with air, 78\% patients given oxygen at $12 \mathrm{~L} / \mathrm{min}$ via reservoir mask for $15 \mathrm{~min}$ had a reduction in pain at $15 \mathrm{~min}$ as compared with $20 \%$ for those breathing air at $12 \mathrm{~L} / \mathrm{min}(\mathrm{p}<0.001) .{ }^{354}$ In a further study, there was a complete or substantial reduction in pain at $30 \mathrm{~min}$ in $56 \%$ of patients (nine participants) in $80 \%$ or more of their cluster headaches when given oxygen at $6 \mathrm{~L} / \mathrm{min}$ for up to 15 min from a reservoir mask compared with $7 \%$ on air. ${ }^{352}$

Evidence statement

- Oxygen therapy can provide relief from cluster headache (evidence level 1-).

Recommendation

F18: For patients with cluster headaches, oxygen should be administered using a flow of at least $12 \mathrm{~L} / \mathrm{min}$ from a reservoir mask and home oxygen should be provided (grade D).

\subsection{Obstetric emergencies and labour}

The use of oxygen has been recommended during many obstetric emergencies and, in particular, for collapse related to haemorrhage, pulmonary embolism, eclampsia or amniotic fluid 
embolism. Severe pre-eclampsia and eclampsia may occasionally present with pulmonary oedema and this can occur in the antenatal or postnatal periods. Medical problems such as pneumonia or acute exacerbations of asthma are not uncommon during pregnancy. Peripartum cardiomyopathy is rare but may present with heart failure in the postnatal period. Major trauma is increasingly common, particularly related to road traffic accidents.

The use of oxygen during pregnancy should follow the same general principles as the use of oxygen for other patients. Pregnant women suffering major trauma or severe hypoxaemia should be started on high-concentration oxygen via a non-rebreathing reservoir mask and those with milder hypoxaemia can use nasal cannulae or a simple face mask or Venturi mask to achieve an oxygen saturation of $94-98 \%$ in most cases. Risk factors for hypercapnia are uncommon among women of child-bearing age but morbid obesity is an increasing problem in the UK population and is associated with risk of hypercapnia (see section 8.12.4). Morbidly obese pregnant women with hypoxaemia are at risk of hypercapnia and should have a target range of $88-92 \%$ pending availability of blood gas results. Pregnant women who are fully conscious with no cardiovascular compromise may be managed in the sitting position or if lying down should use the full left lateral position. If an undelivered woman is hypoxaemic with reduced consciousness level or needing respiratory or cardiovascular support or CPR, she should be managed with left lateral tilt applied. This will improve cardiac output and may also facilitate breathing for mechanical reasons. As an alternative, the patient can be left lying relatively flat and the uterus can be displaced manually (preferably to the left) in order to relieve aortocaval compression. This will usually require additional help and is uncomfortable.

Oxygen was commonly given as part of the treatment for many obstetric emergencies. However, it is recommended that, when oxygen is administered during pregnancy or labour, clinicians should aim to achieve normoxaemia (saturation 94-98\%). There is no randomised trial evidence to suggest that maternal 'hyperoxaemia' is beneficial to mother or fetus. A 2008 review of the use of oxygen during intrauterine resuscitation concluded as follows: "Caution should be exercised when considering maternal oxygen administration as a first-line intrauterine resuscitation measure until more data are available, reserving its use after other measures have been unsuccessful in resolving the non-reassuring fetal heart rate pattern., ${ }^{356}$

Oxygen is often given for short periods when acute fetal compromise is suspected in labour in the hope of increasing oxygen delivery to the fetus. A Cochrane review found no trials addressing the use of oxygen for fetal compromise but the authors identified two trials of prophylactic oxygen in labour which reported a statistically significant increase in the incidence of cord blood acidosis $\left(\mathrm{pH}<7.20\right.$ or $\left.\left[\mathrm{H}^{+}\right]>63 \mathrm{nmol} / \mathrm{L}\right)$ in the oxygenation group (relative risk (RR) 3.5, 95\% CI 1.34 to 9.19). ${ }^{357}$ A small $(n=56)$ randomised trial in Washington, USA reported in 2012 that the delivery room resuscitation rate was $20 \%$ for infants of mothers randomised to $2 \mathrm{~L}$ of nasal oxygen for at least $30 \mathrm{~min}$ before delivery compared with $0 \%$ in those receiving air from a nasal cannula $(\mathrm{p}=0.03) .{ }^{358}$ This study reported cord blood acidosis in just one participant in each group. When this study is added to the Cochrane review, the reported prevalence of cord blood acidosis in three published studies was $18 / 151(12 \%)$ in those randomised to oxygen therapy and 6/140 (4\%) when air was used throughout labour. A recent clinical opinion paper included a review of the two randomised trials assessing use of maternal oxygen supplementation in labour. ${ }^{359}$ These authors also noted an absence of data confirming fetal benefit and the possibility that increased free radical activity may be harmful. The authors of this 2014 paper conclude that appropriately powered randomised trials are still required. Until then, oxygen supplementation should be reserved for confirmed maternal hypoxaemia in labour, and should not be considered an appropriate intervention for nonreassuring fetal status.

This fits with the recommendation that pregnant women with evidence of hypoxaemia should have their blood oxygen saturation maintained in the normal range (94-98\%) using supplemental oxygen only when required to achieve this effect. This applies before or during labour as well as in the immediate postnatal period. The causes of maternal hypoxaemia may include trauma, pre-existing or new medical conditions as well as pregnancy-specific complications. In all of these situations the aim should be normoxaemia (saturation 94-98\%).

A Cochrane review of the use of supplemental oxygen for caesarean section during regional analgesia concluded as follows: "Current evidence suggests that supplementary oxygen given to healthy term pregnant women during elective caesarean section under regional anaesthesia is associated with higher maternal and neonatal oxygen levels (maternal $\mathrm{SpO}_{2}, \mathrm{PaO}_{2}$, $\mathrm{UaPO}_{2}$ and $\mathrm{UvPO}_{2}$ ) and higher levels of oxygen free radicals. However, the intervention was neither beneficial nor harmful to the neonate's short-term clinical outcome as assessed by Apgar scores." 360

A randomised trial of postpartum oxygen inhalation concluded that this practice is not beneficial for reducing vaginal blood loss during the third and fourth stages of labour. ${ }^{361}$

Evidence statements

- There have been no trials comparing different oxygen regimens in acutely unwell pregnant women so management should be based on the oxygen levels that are believed to be optimal in other groups of acutely unwell adults (evidence level 4).

- When women in late pregnancy (ie, above 20 weeks) lie flat on their backs, aortocaval compression by the gravid uterus reduces maternal cardiac output. This is improved by using left lateral tilt or manual displacement of the uterus. Placing the patient in the full left lateral position is another alternative (evidence level 3).

- Although maternal oxygen therapy can increase fetal oxygen levels, there are limited data regarding its risks or possible benefits to the mother, fetus and newborn (evidence level 4)

\section{Recommendations}

H1: Women who suffer from major trauma, sepsis or acute illness during pregnancy should receive the same oxygen therapy as any other seriously ill patients, with a target oxygen saturation of $94-98 \%$. The same target range should be applied to women with hypoxaemia due to acute complications of pregnancy (eg, collapse related to amniotic fluid embolus, eclampsia or antepartum or postpartum haemorrhage) (grade D).

H2: Women with underlying hypoxaemic conditions (eg, heart failure) should be given supplemental oxygen during labour to achieve an oxygen saturation of $94-98 \%$ unless they are at risk of hypercapnic respiratory failure (target range 8892\%) (grade D).

H3: Pregnant women who are fully conscious with no cardiovascular compromise may be managed in the sitting position or if lying down should use the full left lateral position (grade D).

H4: Pregnant women above 20 weeks gestation (uterine fundus at or above the level of the umbilicus) who are at risk of developing associated cardiovascular compromise (eg, trauma, vaginal bleeding, etc) should be positioned to avoid aortocaval compression by using left lateral tilt, manual uterine 
displacement or by placing them in a full left lateral position (grade D).

H5: Women who are more than 20 weeks pregnant with evidence of bypoxaemia associated with reduced consciousness or those requiring respiratory or cardiovascular support or CPR should be managed with left lateral tilt or manual uterine displacement (ideally to the left) to improve cardiac output and oxygen delivery (grade D).

H6: The use of oxygen supplementation during intrauterine fetal resuscitation during labour was widespread in the past but there is no evidence of benefit. There is weak evidence of harm to the fetus if supplemental oxygen is given for long periods during uncomplicated labour. Overall, the use of oxygen during labour is only required when there is evidence of maternal hypoxaemia (oxygen saturation <94\%) (grade D).

\subsection{Use of oxygen in postoperative and perioperative care, including PCA device use}

8.15.1 Oxygen use, pulse oximetry and the incidence of hypoxaemia in the postoperative period

Postoperative breathlessness and hypoxaemia can develop due to a variety of complications including atelectasis, pneumonia and pulmonary embolism. The use of opioid analgesia commonly used for moderate-to-severe pain can lead to and exacerbate respiratory failure, which can potentially be life-threatening. Historically, it has been common practice for postoperative patients to receive routine supplemental oxygen for a period ranging from hours to days after surgery to prevent hypoxaemia, particularly those receiving opioid analgesia. High-risk patients undergoing major surgery are best managed in an ICU and HDU settings which facilitate the continuous monitoring of $\mathrm{SpO}_{2}$ and usually end tidal $\mathrm{CO}_{2}$ (this is standard in ventilated patients). Regular ABG analysis using arterial lines is also available in ICU and HDU settings. This allows oxygen therapy to be administered in a dose sufficient to relieve hypoxaemia without causing hyperoxia.

However, the majority of postoperative patients are managed on general surgical wards where continuous monitoring is not possible. The question of whether all postoperative patients should receive supplemental oxygen routinely is debatable and there is lack of robust evidence from RCTs to either support or refute its use.

A number of observational studies have documented a high incidence of postoperative hypoxaemia. ${ }^{182}$ 362-364 A Cochrane review of 22992 patients reported on the incidence of hypoxaemia in the perioperative and postoperative period. ${ }^{181}$ Unsurprisingly, it is difficult to draw any conclusions on the overall incidence of hypoxaemia due to the variation in population groups, operative procedure and anaesthetic modality.

This same Cochrane review included data from five trials in which patients were randomised to pulse oximetry monitoring or no pulse oximetry during and after surgery. ${ }^{181}$ The review showed that pulse oximetry leads to a 1.5 -fold to 3 -fold reduction in perioperative hypoxaemia. However, while pulse oximetry led to the detection of hypoxaemia and interventions to correct it, there was no difference in complication rates between the two groups. Additionally postoperative cognitive function, length of hospital stay and mortality was the same in both groups. The authors suggested that correcting modest hypoxaemia, by increasing the blood oxygen saturation from marginal to satisfactory may confer no benefit to the patient. However, only small numbers of studies were included and further work is required for clarification.
The incidence of postoperative hypoxaemia demonstrated in randomised trials is again, variable. Gift et $a l^{366}$ found no clinically significant difference in $\mathrm{SpO}_{2}$ in nearly 300 postoperative patients randomised to four different treatments including oxygen $4 \mathrm{~L} / \mathrm{min}$ via nasal cannulae, $40 \%$ oxygen via mask, nurse-led hyperinflation and no treatment. ${ }^{365}$ Canet et $a l^{365}$ found that the incidence of hypoxaemia was higher after breathing air compared with $35 \%$ oxygen and the incidence increased if the patients were older or if they received general anaesthesia. $^{366}$

Given the potential to develop hypoxaemia and the inability to continuously monitor oxygenation in most postoperative patients, is it safer to routinely administer supplemental oxygen to all patients to prevent hypoxaemia? The major disadvantage to this approach is that while supplemental oxygen will correct hypoxaemia it can mask the ability to detect hypoventilation. ${ }^{228}$ Additionally, recently, Niesters et al ${ }^{107}$ have shown that hyperoxia has an additive effect on opioid-induced respiratory depression. Furthermore, there is a large body of evidence showing hyperoxia is associated with haemodynamic alterations which may increase myocardial ischaemia and impair cardiac performance (see section 8.13.1). A recent randomised trial of 340 patients postcardiac surgery administering nasal high-flow oxygen compared with usual care (oxygen to keep $\mathrm{SpO}_{2}>93 \%$ ) showed no improvement in postoperative oxygenation. ${ }^{367}$ However, patients in the high-flow group were less likely to require escalation of respiratory support at any time during the study (47 patients in the high-flow group (27.8\%) compared with 77 patients $(45 \%)$ in the standard care group (OR 0.47 , $95 \%$ CI 0.29 to $0.7, p<0.001)$. The authors hypothesised that the low-level airway pressure support provided by the nasal high-flow system may have influenced this outcome.

In a recent editorial, Martin and Grocott ${ }^{368}$ highlight the need to re-evaluate the unrestricted use of oxygen by anaesthetists. They discuss the relationship between oxygen therapy in perioperative care, critical care and resuscitation, highlighting the lack of clinical benefit from hyperoxaemia and the potential to lead to poor outcomes. They propose a strategy of 'precise control of arterial oxygenation' in which oxygen is administered to a defined target, for example, $\mathrm{PaO}_{2}$ of $8-10 \mathrm{kPa}$ or $\mathrm{SaO}_{2} 88-$ $92 \%$ thus avoiding potential harm from hypoxaemia and hyperoxaemia and improving clinical outcomes. These recommendations are in keeping with this present guideline.

The 'routine' use of supplemental oxygen postoperatively, therefore remains controversial. ${ }^{76} \quad 181 \quad 369-372$ More evidence from RCTs is required. Regular, but not necessarily, continuous oxygen saturation monitoring is mandatory. In keeping with the 2004 SIGN guideline on postoperative care which recommends maintaining an oxygen saturation above $92 \%$ for postoperative patients, this present guideline recommends administering oxygen to maintain a target saturation of $94-98 \%$ for most patients, and a target saturation of $88-92 \%$ in those patients at risk of hypercapnic respiratory failure. ${ }^{370}$

Evidence statement

- Pulse oximetry in the perioperative period leads to a significant reduction in perioperative hypoxaemia. However, identification and correction of mild or moderate hypoxaemia with pulse oximetry does not lead to a reduction in complication rates, length of stay or mortality (evidence level $1+$ ).

Good practice points

$\checkmark$ A target saturation of $94-98 \%$ is recommended for most surgical patients except those at risk of hypercapnic respiratory failure when a range of $88-92 \%$ should be achieved. 
$\checkmark$ Pulse oximetry monitoring is recommended for postoperative patients despite the lack of evidence from randomised studies.

\subsubsection{Patient-controlled analgesia}

One of the commonest indications for anaesthetists to prescribe supplemental postoperative oxygen therapy is to help prevent hypoxaemia when PCA is used. In two reviews, the incidence of postoperative hypoxaemia in patients receiving PCA was found to be $11.5 \%$ and $15.2 \%$. $^{373} 374$

However, Cashman and Dolin's ${ }^{373}$ systematic review comparing the effects of acute postoperative pain management on respiratory function found PCA did not lead to more respiratory events when compared with intramuscular analgesia and epidural anaesthesia. They reviewed 165 studies used a variety of indicators for respiratory depression including hypoventilation, hypercarbia, oxygen desaturation and requirement for naloxone. When using respiratory rate as a measure of respiratory depression, there was no difference between all three modalities of analgesia. However, when oxygen desaturation was used as an indicator for respiratory depression the incidence of desaturation in the intravenous PCA group was significantly lower (mean $11.5 \%$ (95\% CI 5.6\% to 22\%)) compared with epidural analgesia (mean $15.1 \%$ (95\% CI $5.6 \%$ to $22 \%$ )) and intramuscular analgesia (mean 37\% (95\% CI $22.6 \%$ to $45.9 \%$ )). This is perhaps surprising when PCA use is the commonest indication for supplemental oxygen.

Importantly, while administering supplemental oxygen corrects hypoxaemia, it has the ability to mask respiratory depression by delaying the onset of desaturation. ${ }^{102} 107$ A study using transcutaneous carbon dioxide monitoring via ear lobe probe (TOSCA) compared postoperative patients using epidural analgesia and patients using morphine via PCA infusion pump. ${ }^{375}$ All patients received oxygen $4 \mathrm{~L} / \mathrm{min}$ in the postoperative period. Significant desaturations were not observed in either group. However, the PCA group had a higher median $\mathrm{PtCO}_{2}$, lower respiratory rate and longer hypercarbia time as detected by the TOSCA. This study demonstrates that transcutaneous carbon dioxide monitoring is useful in detecting opioid-induced respiratory depression. It also shows that hypoventilation is common when PCA analgesia is administered. However, outside the operating theatre and intensive care where only pulse oximetry is available, the use of supplemental oxygen may mask hypoventilation. For this reason, oxygen should be administered to correct hypoxaemia rather than prevent it. A target saturation of $94-98 \%$ is recommended in most patients except those at risk of hypercapnic respiratory failure when a range of $88-92 \%$ should be achieved.

Niesters et $a l^{107}$ have recently demonstrated in a proof-of-concept study that hyperoxaemia has an additive effect on opioid-induced respiratory depression. In a study of 20 healthy volunteers, there was steep reduction in minute ventilation and increase in end-tidal $\mathrm{CO}_{2}$ when breathing 50\% oxygen compared with breathing air while receiving a remifentanyl infusion. Additionally apnoeic episodes were significantly higher while breathing oxygen. An audit of blood gases from 4866 patients at a university hospital has shown that hypercapnia was commoner than hypoxaemia in blood gas samples from surgical wards, a surgical HDU, theatre and the ICU as well as on medical wards and many of these hypercapnic patients had respiratory acidosis. ${ }^{42}$

In the absence of large RCTs investigating the effects of PCA and the effects of hyperoxaemia, oxygen should be administered to correct hypoxaemia rather than prevent it. A target saturation of $94-98 \%$ is recommended in most patients except those at risk of hypercapnic respiratory failure when a range of $88-92 \%$ should be achieved.

Good practice points

$\checkmark$ Patients using PCA should have two-hourly oximetry observations because of the risk of hypoxaemia. Oxygen should be administered to keep patients within the appropriate target saturation range.

$\checkmark$ A target saturation of $94-98 \%$ is advised in most patients having PCA except those at risk of hypercapnic respiratory failure when a range of $88-92 \%$ should be achieved.

\subsubsection{The role of hyperoxaemia in reducing postoperative complications}

High levels of inspired oxygen (eg, 80\%) in the perioperative and postoperative periods have in the past, been proposed to improve clinical outcomes including a reduction in the incidence of surgical site infection. The rationale for its use being that increased tissue $\mathrm{PO}_{2}$ within surgical wounds might enhance neutrophil killing capacity resulting in reduced rates of infection. Earlier meta-analysis supported this hypothesis; ${ }^{376} 377$ however, three RCTs have been published subsequently and do not concur with these findings. ${ }^{378-380}$

Two recent meta-analysis again show conflicting results: Togioka $e a^{381}$ included seven randomised trials and found no overall benefit of hyperoxia in reducing surgical site infection. However, a statistically significant benefit was demonstrated in subgroups undergoing general anaesthesia and colorectal surgery. Hovaguimian et $a l^{382}$ included nine randomised trials in their meta-analysis and concluded that high perioperative oxygen did reduce the risk of perioperative surgical site infection. Both these meta-analyses included the largest RCT in this area (the PROXI trial), including 1400 patients, which showed no benefit in the administration of $80 \%$ oxygen compared with $30 \%$ oxygen in the risk of developing surgical site infection after abdominal surgery. ${ }^{379}$ Long-term follow-up of patients with cancer within the PROXI study revealed a $45 \%$ increase in long-term mortality in the high $\mathrm{FiO}_{2}$ group (HR was $1.45 ; 95 \%$ CI 1.10 to $1.90 ; \mathrm{p}=0.009) .{ }^{383}$ Kurz et $a l^{384}$ reported in 2015 that supplemental oxygen did not reduce surgical site infection risk among patients having colorectal resections.

Hyperoxia has also been proposed to reduce the incidence of postoperative nausea and vomiting via a number of mechanisms including the prevention of subtle intestinal ischaemia reducing serotonin release and the reduction of dopamine release in the carotid bodies. This has been extensively investigated with conflicting evidence from RCTs. Two recent systematic reviews and meta-analyses show differing results. ${ }^{382} 385$ Orhan-Sungur et $a l^{385}$ included data from 10 studies and showed no overall benefit in hyperoxaemia reducing the risks of postoperative nausea and vomiting. Hovaguimian et $a l^{382}$ included data from 11 studies and showed high concentrations of oxygen provided a significant protective effect in a subgroup of patients who received inhalation anaesthetics without prophylactic antiemetics. However, overall there was no benefit in the composite end point postoperative nausea and vomiting.

Experimental data have shown hyperbaric oxygen improves anastomotic integrity in animal models. ${ }^{386}{ }^{387}$ Recently, 72 patients who underwent elective open infraperitoneal anastomosis for rectal cancer were randomised to $30 \%$ or $80 \%$ oxygen at induction and for 6 hours postoperatively. ${ }^{388}$ The risk of anastomotic leak was $46 \%$ lower in the $80 \% \mathrm{FiO}_{2}$ group (RR 0.63 ; $95 \%$ CI 0.42 to 0.98 ) versus the $30 \% \mathrm{FiO}_{2}$ group. The same group has shown similar results in patients with 
oesophagojejunal anastomosis when the risk of anastomotic leak was $49 \%$ lower in the $80 \% \mathrm{FiO}_{2}$ group (RR $0.61 ; 95 \%$ CI 0.40 to 0.95 ) versus the $30 \% \mathrm{FiO}_{2}$ group. ${ }^{389}$ However, the numbers of patients in both studies in each treatment arm were small and further work is required from other investigators to validate this finding, especially in view of the concerns of Meyhoff et al ${ }^{383}$ that high-concentration oxygen may increase medium-term mortality in patients having cancer surgery.

\section{Evidence statements}

- The possible effects of hyperoxaemia in the perioperative and postoperative period remain controversial and may or may not reduce overall surgical site infection. However, there may be benefit in subgroups of patients undergoing general anaesthesia and colorectal surgery for non-malignant disease (evidence level 1-).

- Hyperoxaemia in the perioperative and postoperative period does not substantially reduce the incidence of postoperative nausea and vomiting but meta-analyses have come to conflicting conclusions (evidence level 1-).

- Hyperoxaemia in the perioperative and postoperative period has been shown to improve anastomotic integrity in patients undergoing gastric and colorectal surgery (evidence level 1-). Recommendation

J1: Hyperoxaemia is not recommended routinely in the perioperative and postoperative period to reduce the incidence of postoperative nausea and vomiting (grade D).

Good practice point

$\checkmark$ There is conflicting evidence concerning the balance of potential benefits and risks of perioperative hyperoxaemia to reduce the risk of surgical site infection in elective surgery and there is no evidence for this practice in patients having emergency surgical procedures. More trials are required for specific procedures and more information is required concerning long-term mortality risks to patients with cancer. In the meantime, oxygen should not be used for this indication outside of clinical trials.

\subsection{Use of oxygen during endoscopy and other procedures involving conscious sedation}

Conscious sedation may be defined as "a technique in which the use of a drug or drugs produces a state of depression of the central nervous system enabling treatment to be carried out, but during which verbal contact with the patient is maintained throughout the period of sedation. The drugs and techniques used to provide conscious sedation should carry a margin of safety wide enough to render loss of consciousness unlikely."390 Conscious sedation is a common hospital procedure in a variety of settings from the endoscopy suite to the emergency department. It is performed by anaesthetists, physicians, trained nurses and operating department practitioners. The most commonly used sedative agents are those belonging to the benzodiazepine and opioid classes and increasingly, propofol.

During fibre-optic bronchoscopy reduction in $\mathrm{SaO}_{2}$ is a

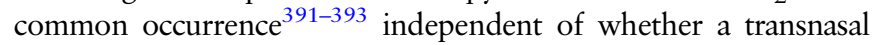
or transoral approach is selected. ${ }^{394}$ There are several contributing factors including the effect of central respiratory depressant drugs, partial physical occlusion of the airway by the bronchoscope, prolonged use of airway suction, as well as V/Q mismatch produced by instillation of anaesthetic solution and lavage fluids in the airways. ${ }^{395-397}$ One small non-blinded randomised study showed no significant influence of patient positioning (supine vs semirecumbent) on the desaturation observed during fibre-optic bronchoscopy, ${ }^{393}$ although a further study has shown a higher rate of desaturation in the sitting as opposed to supine position. ${ }^{398}$
Milman et $a l^{391}$ demonstrated that following benzodiazepine sedation, without supplemental oxygen, $38 \%$ of patients desaturate $\left(\mathrm{SpO}_{2}<90 \%\right)$ before bronchoscopy, $80 \%$ during and $60 \%$ following the procedure. Thus, all patients undergoing fibre-optic bronchoscopy should be routinely monitored using transcutaneous pulse oximetry which provides an accurate noninvasive method for assessing hypoxaemia during the sedation, procedure and recovery stages.

There are variable data available on the effect of hypoxaemia on the incidence of significant complications including cardiac arrhythmia during fibre-optic bronchoscopy. In an early prospective study minor rhythm abnormalities were frequently noted, with more major rhythm abnormalities more commonly seen in patients who developed hypoxaemia following the procedure. ${ }^{399}$ All arrhythmias were self-limiting and did not lead to haemodynamic compromise. This finding was reiterated in a further study which again showed a noted association between hypoxaemia and the development of cardiac arrhythmia. ${ }^{392}$ In contrast, Lundgren $e t a l^{395}$ in a small study of 10 patients with restrictive lung disease secondary to pulmonary fibrosis demonstrated that although prolonged airway suctioning lead to a reduction in $\mathrm{PaO}_{2}$ there was no significant associated increased incidence of cardiac arrhythmia. Further studies have shown no increase in arrhythmias during fibre-optic bronchoscopy, with similar rates of minor arrhythmias to that seen during lung function testing. ${ }^{391} 394$

The degree and duration of hypoxaemia may be ameliorated by oxygen supplementation provided via a nasal or pharyngeal catheter. $^{391393}$ In a large 6-year retrospective study $(n=1051)$ it was demonstrated that routine use of oxygen on a prophylactic basis was not required during fibre-optic bronchoscopy even when conscious sedation is performed, as most desaturations observed were transient and thus not requiring specific intervention. ${ }^{400}$ The requirement for supplemental oxygen is, however, known to increase in relation to falling $\mathrm{FEV}_{1}$ or PEF. Jones and O'Driscoll ${ }^{400}$ showed that $35 \%$ of those with an $\mathrm{FEV}_{1}<1 \mathrm{~L}$ required administration of oxygen, $14 \%$ with an $\mathrm{FEV}_{1}$ of 1.0 $1.5 \mathrm{~L}$ and $7 \%$ with an $\mathrm{FEV}_{1}>1.5 \mathrm{~L}$. However, requirement for supplemental oxygen remains variable, ranging from 5\% to $32 \%$ and thus needs to be assessed on an individual basis. ${ }^{400} 401$

Oxygen supplementation should thus be used when desaturation is significant $\left(\mathrm{SpO}_{2}<90 \%\right.$, change of $\left.\mathrm{SpO}_{2}>4 \%\right)$ and prolonged $(>1 \mathrm{~min})$ to reduce the risk of hypoxaemia-associated complications. ${ }^{391} 392399400402$ The arterial oxygenation target should be dependent on the individual patient, a $\mathrm{SpO}_{2}$ of 94 $98 \%$ being suitable for the majority of patients, except those at risk of hypercapnic respiratory failure where an appropriate target is a $\mathrm{SpO}_{2} 88-92 \%$.

Reduction in transcutaneous $\mathrm{SaO}_{2}$ has also been reported during and following both upper ${ }^{403-409}$ and lower ${ }^{408-410}$ GI endoscopy, particularly during more complicated therapeutic procedures such as endoscopic retrograde cholangiopancreatography. ${ }^{409} 411412$ A small observational study showed more marked desaturation in those patients with a pre-existing requirement for long-term oxygen therapy. ${ }^{412}$ Hypoxaemia is likely to be multifactorial, as is the case with bronchoscopic procedures, a consequence of sedation and the physical distortion of the airway produced by passage of the endoscope. Thus, as with fibre-optic bronchoscopy it is essential to ensure adequate facilities for monitoring of transcutaneous oxygen saturations at all stages throughout the procedure.

Hypoxaemia during GI endoscopy may be more frequently observed in individuals with pre-existing cardiorespiratory disease or those undergoing complicated therapeutic 
procedures, especially if heavily sedated. ${ }^{292} 293295296300301$ Although the use of oxygen therapy on a prophylactic basis is suggested in all those patients receiving conscious sedation in the current guidelines of the British Society of Gastroenterology, ${ }^{301}$ there are, however, no recognised studies analysing whether routine use of prophylactic oxygen decreases or increases the risks of GI endoscopy even in at-risk patients. There is a risk that routine oxygen use might actually mask the development of respiratory failure in such patients because the patient could develop hypercapnia and carbon dioxide narcosis despite having satisfactory oxygen saturation measurements. Such patients require careful continuous oxygen saturation monitoring and clinical observation throughout, and facilities for oxygen administration should be readily available in the event of significant desaturation as discussed in section 7 .

Limited data are available on the use of oxygen during other procedures involving conscious sedation. Invasive cardiac procedures such as cardiac catheterisation and angioplasty have been associated with arterial oxygen desaturation in a significant proportion of patients, particularly those with recognised cardiorespiratory disease, and can be corrected by supplemental oxygen administration. ${ }^{413}$ In an emergency department setting, procedures requiring conscious sedation may also be associated with hypoxaemia; however, use of oxygen in a prophylactic manner has not been demonstrated to reduce the incidence of hypoxaemic events, although these studies were small and likely to be underpowered to this end. ${ }^{414} 415$

$\mathrm{SaO}_{2}$ monitoring is indicated during the administration of sedation, procedure and recovery period in all procedures involving conscious sedation. Significant caution should be taken with both the administration of sedation and supplemental oxygen in patients with known resting hypercapnia or significant lung disease who are at risk of hypercapnic respiratory failure. It is crucial to ensure sufficient clinical assessment of patients at all times. Monitoring of transcutaneous carbon dioxide levels and capnography in additional to standard monitoring may be of use, particularly in high-risk patients and those receiving moderate or deep sedation to identify early signs of respiratory depression. ${ }^{416} 417$

Evidence statements

- Hypoxaemia is a common observation during fibre-optic bronchoscopy related to use of central depressant drugs, partial airway occlusion, use of airway suction, patient positioning and instillation of fluid into the airways. There is a noted relationship between reduced $\mathrm{FEV}_{1}$ and PEF and increased occurrence of hypoxaemia (evidence level 4).

- Hypoxaemia is also noted during both upper and lower GI endoscopy and other procedures involving conscious sedation (evidence level 2+).

- The use of supplemental oxygen via nasal or pharyngeal catheter can ameliorate hypoxaemia (evidence level 2+).

- Oxygen supplementation should be provided to patients with persistent reduction in $\mathrm{SaO}_{2}\left(\mathrm{SpO}_{2}<90 \%\right.$ or change $>4 \%$, for $>1 \mathrm{~min}$ ) (evidence level 4).

\section{Recommendations}

J2: All procedures involving conscious sedation warrant routine continuous monitoring of oxygen saturation via pulse oximetry prior to and during the procedure, and in the recovery period, particularly fibre-optic bronchoscopy and upper GI endoscopy where a reduction in $\mathrm{SaO}_{2}$ is common, particularly with concurrent use of sedation (grade $\mathrm{C}$ ).

J3: Significant arterial oxygen desaturation $\left(\mathrm{SpO}_{2}<90 \%\right.$ or fall of $4 \%$ or more that is prolonged ( $>1 \mathrm{~min}$ during endoscopy procedures)) should be corrected by supplemental oxygen with the aim of achieving target oxygen saturations of $94-98 \%$, or $88-92 \%$ in those at risk of hypercapnic respiratory failure (grade D).

J4: Complicated upper GI endoscopy or procedures in patients with cardiorespiratory comorbidity are especially likely to lead to hypoxaemia and may also lead to hypercapnia, especially if the patient is heavily sedated. It is recommended that blood gases should be measured if such patients should require prolonged oxygen administration. The routine administration of oxygen is not recommended as it may delay the recognition of respiratory failure (grade $\mathrm{D}$ ).

J5: Constant clinical assessment of the patient is crucial at all stages of conscious sedation procedures and monitoring of capnography or transcutaneous carbon dioxide levels may be a useful adjunct to identify early respiratory depression (grade D).

J6: During the recovery period after procedures requiring conscious sedation, supplemental oxygen should be titrated to achieve target saturations of $94-98 \%$ in most patients and $88-92 \%$ in those at risk of hypercapnic respiratory failure (see section 10.5.1) (grade D).

\subsection{Use of oxygen in palliative care settings}

In 2004, a working party of the Association of Palliative Medicine Scientific Committee set out to examine the evidence concerning the use of oxygen for the palliation of breathlessness in COPD, advanced cancer and chronic heart failure. ${ }^{418}$ They found that there was evidence that oxygen may have a useful role in the palliation of breathlessness. ${ }^{419} 420$ However, the conclusion of most subsequent studies ${ }^{147} 148150421$ and systematic reviews ${ }^{159} 422-424$ is that there is no difference between oxygen or air in relief of breathlessness, although the intervention with either seems to induce some sense of relief of symptoms. There is general consensus that facial cooling (eg, by hand-held fan) of second and third branches of trigeminal/vagal nerve reduces the sensation of breathlessness. ${ }^{425}$ These mechanisms are believed to be the reasons why both air and oxygen seem to work equally.

In addition, there is also the question of the relationship of hypoxaemia to the sensation of breathlessness. There does not seem to be a clear relationship between the two as it has been shown that occurrence of hypoxaemia (or absence of hypoxaemia) has no relationship to relief of breathlessness in palliative care patients. ${ }^{426}$

Breathlessness in terminally ill patients is a complex multifaceted problem. The relief of this distressing symptom should be individualised in each patient. The evidence of benefit from opioids and non-pharmacological treatments is stronger than the evidence of benefit from oxygen, especially for nonhypoxaemic patients ${ }^{426} 427$ (evidence level $2+$ ). Simple devices like hand-held fans may be tried before more complex methods (eg, oxygen and its attached equipment) are tried. There is concern in the clinical community that dying patients may become psychologically dependent on oxygen and become afraid to let go of the equipment even when clearly dying. Face masks can be intrusive and may be perceived as a barrier by the family members between them and the patient. Campbell and colleagues have shown that, when death is imminent, in the absence of respiratory distress, oxygen should not be routinely given even when severe hypoxaemia is present. Among those already receiving oxygen, it was possible to discontinue it in $90 \%$ without causing distress. ${ }^{428}$

Previous clinical recommendations for the use of oxygen in palliative care were based on the recommendations from the 
working party of the Association of Palliative Medicine Science Committee in $2004^{418}$ but most publications since that date have cast doubt on the value of oxygen therapy for patients with advanced cancer or heart failure unless the patient is hypoxaemic. For some conditions such as advanced pulmonary fibrosis requiring palliative care, the potential benefits of oxygen for non-hypoxaemic patients at rest have not been addressed in clinical trials although many patients with this condition desaturate on exertion and benefit from ambulatory oxygen therapy. Readers should refer to the BTS Home Oxygen guideline for guidance about the medium-term use of oxygen by palliative care patients in the home setting. ${ }^{13}$

Evidence statements

- Breathlessness is a common symptom among palliative care patients but there is little evidence that the degree of breathlessness is related to the oxygen saturation (evidence level 2 $+)$.

- Most recent studies have shown that there is no difference between oxygen and air in the relief of breathlessness in nonhypoxaemic palliative care patients (evidence level $1+$ ).

- There is contradictory evidence concerning the possible benefits of oxygen in hypoxaemic palliative care patients, most studies were negative (evidence level $2+$ ).

- Recent studies suggest that alternative treatments such as opioids or non-pharmacological therapy such as a hand-held fan or treatment of anxiety may be more beneficial (evidence level $2+$ ).

\section{Recommendations}

K1: Oxygen use in palliative care patients should be restricted to patients with $\mathrm{SpO}_{2}$ consistently $<90 \%$ or patients who report significant relief of breathlessness from oxygen. In nonhypoxaemic patients, opioids and non-pharmacological measures should be tried before oxygen (grade B).

K2: In general, there is no role for the monitoring of oxygen saturation or $\mathrm{PO}_{2}$ in comfort-focused care in the last few days of life. If the patient appears comfortable, oxygen levels are irrelevant and should not influence care (grade D).

Good practice points related to oxygen use in palliative care

Oxygen therapy for the symptomatic relief of breathlessness in palliative care patients is more complex than the simple correction of hypoxaemia. Consider the following issues:

$\checkmark$ Consider early involvement of palliative care specialists and physiotherapists.

$\checkmark$ As breathlessness is a multifactorial sensation, a comprehensive assessment of contributing factors (such as anxiety) should be carried out.

$\checkmark$ Low-dose opioids should be considered because they are effective for the relief of breathlessness in palliative care patients.

$\checkmark$ A trial of a hand-held fan to help relieve breathlessness is recommended prior to trial of oxygen.

$\checkmark$ Oxygen use has to be tailored to the individual and a formal assessment made of its efficacy for reducing breathlessness and improving quality of life for that person.

$\checkmark$ Oxygen therapy should not be continued in the absence of patient benefit or where its disadvantages (eg, discomfort of masks or nasal cannulae, drying of mucous membranes) outweigh any likely symptomatic benefit.

\subsection{Use of helium-oxygen mixtures (Heliox)}

Helium is an inert gas which is lighter and less dense than oxygen and nitrogen which comprise over $99 \%$ the air that we breathe. Helium has been mixed with oxygen (Heliox), usually in a ratio of $80: 20$ or $70: 30$ to treat adults and children with upper and lower airway disease as the reduced gas density can decrease resistance to airflow and therefore the work of breathing in narrowed airways. A 4-year study in a critical care unit reported 92 instances of use (73\% paediatric) with an $\sim 50 / 50$ ratio of upper and lower airway disorders treated by Heliox. ${ }^{429}$

The use of Heliox for upper airway obstruction in adults has not been the subject of formal clinical trials because presentations are rare, the site and nature of airflow obstruction is variable and patients tend to present as acute emergencies where randomisation would be difficult. However, there are case reports of benefit where Heliox has been used with apparent benefit to patients (and no side effects other than temporary alteration in the voice).

Heliox has been used in asthma and COPD, both as an inhaled gas and as the driving gas for nebulised treatments. There is a great deal of uncertainty about possible benefits, partly because the gas itself can affect the calibration and functioning of equipment and this can influence measures of airflow such as peak expiratory flow and $\mathrm{FEV}_{1}$. A systematic review in 2003 concluded that the existing evidence did not provide support for the administration of helium-oxygen mixtures to emergency department patients with moderate-to-severe acute asthma with the caveat that the conclusions were based on between-group comparisons and small studies and the results should be interpreted with caution. ${ }^{430}$ A later review in 2007 and a small randomised trial published in 2011 noted a slight improvement in airflow measures but the clinical significance could not be determined. ${ }^{431} 432$

The use of Heliox in COPD has been less studied than its use in asthma. A retrospective study of 81 patients with AECOPD and respiratory acidosis reported a significant decrease in intubation and mortality in the Heliox group and shorter ICU stay and hospital stay in survivors. ${ }^{43}$ The authors advised (in 2001) that prospective randomised studies were needed and a later meta-analysis (in 2004) concluded that definitive evidence of benefit was lacking. ${ }^{434}$ Heliox has been used with reported benefit in conjunction with non-invasive positive pressure ventilation but that is outside the scope of this guideline.

The use of Heliox as a driving gas for nebulisers is also controversial, partly due to the complex interactions between the driving gas and the inhaled medication and measures of airflow. A systematic review in 2007 concluded that the use of Heliox to drive nebulisers in patients with acute asthma slightly improved airflow measures but it was not possible to determine whether this improved recovery. ${ }^{431}$

Evidence statements

- There is little evidence overall to suggest that Heliox has any benefit over air-oxygen mixtures in asthma or COPD and it is more expensive than oxygen-air mixtures (evidence level 1-).

- There is weak evidence from case reports and clinical practice that a therapeutic trial of Heliox may be reasonable in patients with mechanical upper airway obstruction or postoperative stridor (evidence level 3).

\section{Recommendations}

L1: There is insufficient evidence to support the use of Heliox either as an inhaled gas or as the driving gas for nebuliser therapy in adult patients with acute exacerbations of asthma or AECOPD except as part of randomised clinical trials or in exceptional circumstances (grade D).

L2: A therapeutic trial of Heliox is reasonable in patients with mechanical upper airway obstruction or postoperative stridor (grade D).

L3: Heliox use for patients with asthma or COPD should be considered only in clinical trials or in specialist hands for severe exacerbations that are not responding to standard treatment 
(and in patients with COPD where there are contraindications to intubation) (grade D).

\subsection{Use of CPAP}

The principles and indications for use of CPAP outside of urgent care are beyond the scope of this guideline as are the technical details of CPAP use. Acute CPAP therapy is used commonly in the management of acute pulmonary oedema and many patients who are overweight or long-term users of home CPAP require hospital admission for the management of medical and surgical conditions. Most of these patients should continue to use their own CPAP device during their hospital admission (usually overnight) and some may need to entrain oxygen to maintain their target oxygen saturation range. The use of CPAP in patients in the preoperative and postoperative period to treat or prevent hypoxaemia in patients with sleepdisordered breathing and the use of CPAP to treat pulmonary oedema warrant special consideration.

\subsubsection{Use of CPAP in perioperative care}

Obesity has reached epidemic proportions with more than one billion overweight adults worldwide, of whom at least 300 million are obese (see also section 8.15 ). Obesity predisposes to OSA and OHS. CPAP is the mainstay of medical treatment for patients with OSA. It has been shown to reduce apnoeas and daytime sleepiness as well as reducing sequelae of untreated OSA such as impaired cognition, hypertension, coronary artery disease and cerebrovascular accident. ${ }^{435}$ The incidence of postoperative desaturation and respiratory failure is unsurprisingly high in patients with OSA. ${ }^{436}$ Residual anaesthetic drugs have the potential to weaken the muscles of the upper airway and depress the respiratory drive, thus amplifying the disease. CPAP in the postoperative period has been shown to reduce reintubation and severe respiratory complications after major abdominal surgery in patients without sleep-disordered breathing. ${ }^{437} \mathrm{An}$ observational study of 16 patients with OSA reported a reduction in postoperative complications in patients who used CPAP preoperatively, on extubation and continuously for 24-48 hours after surgery. ${ }^{438}$ The British Obesity and Metabolic Surgery Society (BOMSS) recommend patients with diagnosed sleepdisordered breathing established on CPAP or bi-level positive airway pressure ventilation (BiPAP) who are undergoing surgery, to bring their machines with them and use them in the preoperative and postoperative period. ${ }^{439}$ Patients with OSA or OHS with evidence of desaturation $<88 \%$ in the postoperative period should be started on CPAP or BiPAP rather than supplemental oxygen alone. Supplemental oxygen can be entrained if necessary to maintain the target saturation range, usually $88-$ 92\%.

Caution should be exercised when administering oxygen to all morbidly obese patients even those without a diagnosis of sleep-disordered breathing. One study of 40 morbidly obese patients undergoing laparoscopic bariatric surgery showed the incidence of postoperative hypoxaemia was as high in patients without OSA compared with those with a diagnosis of OSA and supplemental oxygen did not reduce the frequency of oxygen desaturations. ${ }^{440}$

\section{Evidence statements}

- OSA is associated with increased incidence of desaturation, respiratory failure, cardiac events and ICU admission (evidence level 1+).

- CPAP therapy used in the preoperative and postoperative period reduces complications in patients with OSA after surgery (evidence level $2+$ ).

\section{Recommendation}

N1: Patients with diagnosed sleep-disordered breathing established on CPAP undergoing surgery should bring their machines with them and use them in the preoperative and postoperative period. If adequate saturations are not achieved despite CPAP therapy then assess for worsening ventilation with blood gases and oxygen should be entrained to achieve a saturation of 88-92\% (grade D).

\subsubsection{Use of CPAP in acute pulmonary oedema}

Two meta-analysis studies and one large RCT (Gray et $a l^{443}$ ) have provided evidence that CPAP administered with supplemental oxygen has definite measurable physiological and shortterm clinical benefits in pulmonary oedema. ${ }^{441} 442$ The longer term benefits are less clear, in particular any reduction in mortality. The meta-analysis and a prehospital RCT looking at use of CPAP suggest reduced mortality and/or intubation rates. $^{441} 442444$ However, this mortality benefit was not found in the largest and best constructed RCT. ${ }^{443}$ In addition, this study did not report a reduction in the number of intubations.

Evidence statements

- Patients with cardiogenic pulmonary oedema, treated with CPAP and $\mathrm{O}_{2}$ show early improvement in gas exchange when compared with standard treatment (evidence level $1+$ ).

- Reports that short-term mortality and intubation rates are decreased were not confirmed in the largest RCT (evidence level $1+)$.

- The use of CPAP therapy in the prehospital environment may be beneficial to patients with acute pulmonary oedema as it can potentially decrease the need for endotracheal intubation, improve vital signs during transport to hospital and improve short-term mortality (evidence level $1+$ ).

\section{Recommendation}

N2: CPAP with entrained oxygen to maintain saturation 9498\% should be considered as an adjunctive treatment to improve gas exchange in patients with cardiogenic pulmonary oedema who are not responding to standard treatment in hospital care or in prehospital care (grade B).

\section{SECTION 9: EMERGENCY USE OF OXYGEN IN AMBULANCES, COMMUNITY AND PREHOSPITAL SETTINGS}

This section applies to a range of clinical settings to include emergency oxygen use in patients' homes, GP practices or health centres and within an ambulance care setting. Management in some prehospital settings such as a primary care centre or in a paramedic ambulance may be almost identical to hospital management. Readers are referred to section 10 for advice concerning choice of oxygen delivery devices and systems.

Readers are referred to tables 1-4 and charts 1 and 2 for a summary of the key elements of oxygen therapy in common medical emergencies. A brief summary of this section can be downloaded from www.brit-thoracic.org.uk.

Ongoing care at home of chronically hypoxaemic patients is not covered by this guideline. There is little literature on which to base any recommendations when such patients have an acute exacerbation of their condition, but patient safety should be the priority. The NICE guidelines on COPD recommend that patients receiving long-term oxygen and those with an $\mathrm{PaO}_{2}$ of $<7 \mathrm{kPa}$ should be considered for treatment in hospital during exacerbations. ${ }^{1}$

\section{Good practice point}

$\checkmark$ Chronically hypoxaemic patients with a clinical exacerbation associated with a $3 \%$ or greater fall in oxygen saturation on 
their usual oxygen therapy should usually be assessed in hospital with blood gas estimations. $\mathrm{PaO}_{2}$ of $<7 \mathrm{kPa}$ equates to $\mathrm{SpO}_{2}$ below $\sim 85 \%$.

\subsection{Pulse oximetry and availability of oxygen}

It is essential to provide optimal oxygen therapy at the earliest possible opportunity while the acutely breathless patient is being assessed and treated in the community and during transfer to hospital. For most such patients the main concern is to give sufficient oxygen to support their needs. Hypoxaemia can lead to cardiac arrhythmias, renal damage and, ultimately, cerebral damage. However, excessive oxygen therapy can also be dangerous for some patients, especially those with advanced COPD. Target saturation should be used; pulse oximetry is necessary to achieve this. Section 10.4.2 provides advice concerning the choice of oxygen cylinders in primary care practices.

Emergency ambulances and emergency/fast response type vehicles and ambulance service motorbikes and cycles should be equipped with oxygen and oximeters appropriate to the mode of transport. Thus, fast response cars/motorbikes and cycles will require hand-held finger oximeter-type devices and staff initiating oxygen in the home will need a portable or finger oximeter. Pulse oximetry devices used by Community First Responder (CFR) schemes should wherever possible reflect that of the ambulance service to which they are affiliated. Likewise Voluntary Aid Societies (VAS) medical directors are encouraged to discuss the purchase and use of pulse oximeters.

There are many cheap, small, finger oximeters available on the internet, but there has been no thorough evaluation of the accuracy and reliability of these devices. However, they are now being widely used by medical staff in many settings, and by patients on oxygen therapy at home. In the future, having a small oximeter may be as ubiquitous as a stethoscope, reinforcing that oxygen saturations should be the 'fifth vital sign'. Within hospital medicine, the oximeter reading is now established as an essential part of the NEWS. ${ }^{96}$

Evidence statement

- The use of oximeters in acute care is based on physiological data and expert opinion in the absence of randomised trials (evidence level 4).

\section{Recommendations}

T1: Pulse oximetry must be available in all locations where emergency oxygen is being used by healthcare professionals (see also the limitations of using pulse oximetry section 7.1.2) (grade D).

V1: Emergency oxygen should be available in primary care medical centres, preferably using oxygen cylinders with integral high-flow regulators. Alternatively, oxygen cylinders fitted with high-flow regulators (delivering up to $15 \mathrm{~L} / \mathrm{min}$ ) must be used to allow use with reservoir masks (grade D).

V2: Healthcare organisations should take measures to eliminate the risk of oxygen tubing being connected to the incorrect wall oxygen outlet or to outlets that deliver compressed air or other gases instead of oxygen. Air flow meters should be removed from the wall sockets or covered with a designated air outlet cover when not in use. Special care should be taken if twin oxygen outlets are in use (grade D).

\subsection{Clinical assessment by first responder(s) (GP, nurse or ambulance staff)}

It is suggested that the first healthcare professional(s) to encounter an acutely breathless patient should perform an initial 'ABC' assessment, followed by obtaining a quick history from the patient and/or family or friends. Immediate assessment should include recording of pulse rate, respiratory rate and pulse oximetry.

- Clinical assessment of a breathless patient starts with 'ABC' (see recommendation $\mathrm{B} 1$ ).

- A brief history should be taken from the patient or other informant.

- Initial assessment should include pulse and respiratory rate in all cases (see recommendation B1).

- Pulse oximetry should always be measured in patients with breathlessness or suspected hypoxaemia (see recommendation B2).

- Disease-specific measurements should also be recorded (eg, peak expiratory flow in asthma, blood pressure in cardiac disease).

\subsection{Immediate management of hypoxaemic patients}

Having ascertained that the airway is clear, the first responders should start oxygen treatment if the oxygen saturation is below the target. The initial oxygen therapy should follow the general principles given in tables $1-4$ and charts 1 and 2 . There is some evidence that bronchodilator therapy, however given, can cause increased $\mathrm{V} / \mathrm{Q}$ mismatch and reduced blood oxygen levels in acutely ill patients shortly after treatment (see section 10.8.1).

Good practice points

$\checkmark$ The initial oxygen therapy to be used in the various clinical situations is given in tables $1-4$.

$\checkmark$ If there is a clear history of asthma or heart failure or other treatable illness, appropriate treatment should be instituted in accordance with guidelines or standard management plans for each disease.

$\checkmark$ The oxygen saturation should be monitored continuously until the patient is stable or arrives at hospital for a full assessment. The oxygen concentration should be adjusted upwards or downwards to maintain the target saturation range.

$\checkmark$ In most emergency situations, oxygen is given to patients immediately without a formal prescription or drug order. The lack of a prescription should never preclude oxygen being given when needed in an emergency situation. However, a subsequent written record must be made of what oxygen therapy has been given to every patient (in a similar manner to the recording of all other emergency treatment).

\subsection{Patients with known COPD}

A proportion of breathless patients will have COPD. A 2006 Cochrane review of oxygen therapy for COPD in the prehospital setting found no relevant randomised studies but the paper by Austin et $a l^{51}$ in 2010 has shown a reduced mortality in patients with COPD in prehospital care who were managed with titrated oxygen to achieve a target saturation of $88-92 \%$ compared with high-concentration oxygen therapy (RR $0.22) .{ }^{445}$

Audits of emergency admissions in UK hospitals have shown that at least $5 \%$ of all medical admissions and $25 \%$ of breathless or hypoxaemic medical patients who require hospital admission have COPD or other conditions that may place them at risk of hypercapnia. ${ }^{165}$ UK government data list over 100000 hospital admissions due to COPD each year which constitutes $2 \%$ of all emergency admissions to hospitals in England and Wales. ${ }^{446}$ Many of these patients will require carefully titrated oxygen therapy because they are at risk of carbon dioxide retention or respiratory acidosis. In a large UK study, $47 \%$ of patients with exacerbated COPD had $\mathrm{PaCO}_{2}>6.0 \mathrm{kPa}(45 \mathrm{~mm} \mathrm{Hg}), 20 \%$ had respiratory acidosis $\left(\mathrm{pH}<7.35\right.$ or $\left.\left[\mathrm{H}^{+}\right]>45 \mathrm{nmol} / \mathrm{L}\right)$ and $4.6 \%$ 
had severe acidosis $\left(\mathrm{pH}<7.25\right.$ or $\left.\left[\mathrm{H}^{+}\right]>56 \mathrm{nmol} / \mathrm{L}\right){ }^{43}$ Acidosis was more common if the blood oxygen was $>10 \mathrm{kPa}$ $(75 \mathrm{~mm} \mathrm{Hg})$. Plant $e t a l^{43}$ recommended that patients with acute COPD should be maintained within a $\mathrm{PaO}_{2}$ range of $7.3-10 \mathrm{kPa}$ $(55-75 \mathrm{~mm} \mathrm{Hg})$ to avoid the dangers of hypoxaemia and acidosis. Good practice points (section 8.12 and table 3)

$\checkmark$ Patients with COPD should initially be given oxygen via a Venturi $24 \%$ mask at $2-3 \mathrm{~L} / \mathrm{min}$ or Venturi $28 \%$ mask at a flow rate of $4-6 \mathrm{~L} / \mathrm{min}$ or nasal cannulae at a flow rate of 1$2 \mathrm{~L} / \mathrm{min}$.

$\checkmark$ Some patients may benefit from higher flow rates via the Venturi mask (see section 10.2.3). The target oxygen saturation should be $88-92 \%$ in most cases or an individualised saturation range based on the patient's blood gas measurements during previous exacerbations.

\subsection{Patients who should be assumed to have COPD}

One of the challenges faced by the initial clinical response staff is that the diagnosis may be unclear and the patient's medical records or detailed history may not be available. It has been shown that ambulance teams may be aware of a diagnosis of COPD in only $58 \%$ of cases. ${ }^{447}$

The Guideline Group consider that an initial diagnosis of COPD should be assumed if there is no clear history of asthma and the patient is $>50$ years of age and a long-term smoker or ex-smoker with a history of longstanding breathlessness on minor exertion. The diagnosis should be reassessed on arrival at hospital where more information will probably become available, and the $\mathrm{FEV}_{1}$ should be measured unless the patient is too breathless to undertake spirometry.

Good practice point

$\checkmark$ If the diagnosis is unknown, patients $>50$ years of age, who are long-term smokers with a history of chronic breathlessness on minor exertion such as walking on level ground, and no other known cause of breathlessness, should be treated as having suspected COPD for the purposes of this guideline. Patients with COPD may also use terms such as chronic bronchitis and emphysema to describe their condition but may sometimes mistakenly use 'asthma' (see table 3 ).

\subsection{Other patients at risk of hypercapnic respiratory failure with respiratory acidosis}

- Patients who are already on long-term oxygen therapy most of whom will have COPD.

- Patients with fixed airflow obstruction associated with bronchiectasis.

- Any patient with severe kyphoscoliosis or severe ankylosing spondylitis.

- Severe lung scarring from old tuberculosis (especially with thoracoplasty).

- Morbid obesity (BMI $>40 \mathrm{~kg} / \mathrm{m}^{2}$ ).

- Patients with neuromuscular disorders (especially if muscle weakness has led to wheelchair use).

- Any patient on home mechanical ventilation.

- Overdose of opioids, benzodiazepines or other drugs causing respiratory depression.

\subsection{Oxygen alert cards and $24 \%$ or $28 \%$ Venturi masks in patients with COPD (and others at risk of respiratory acidosis) who have had an episode of hypercapnic respiratory failure}

The administration of high oxygen concentrations in acute COPD and other conditions (see section 8.12) leads to worsening of hypercapnic respiratory failure and respiratory acidosis. ${ }^{43}$
Patients with COPD on oxygen therapy with a $\mathrm{PO}_{2}>10 \mathrm{kPa}$ $(75 \mathrm{~mm} \mathrm{Hg})$ are at risk of $\mathrm{CO}_{2}$ retention may be assumed to have excessive oxygen therapy. This is a very common problem, data from the 2008 national COPD audit indicate that $30 \%$ of patients with COPD had received $>35 \%$ oxygen in the ambulance prior to admission and 35\% were still receiving highconcentration oxygen when blood gases were taken in hospital. ${ }^{46}$

If a patient is found to have respiratory acidosis due to excessive oxygen therapy, the oxygen therapy should not be discontinued immediately because the oxygen level will fall significantly over 1-2 min by virtue of the alveolar gas equation (see section 5.2.1), whereas the carbon dioxide level will take much longer to correct itself (see section 5.3.2). In this situation, the oxygen treatment should be stepped down to $28 \%$ or $35 \%$ oxygen from a Venturi mask, or oxygen at $1-2 \mathrm{~L} / \mathrm{min}$ from nasal cannulae depending on oxygen saturation and blood gas results. A saturation target of $88-92 \%$ is recommended for acidotic patients in hypercapnic respiratory failure and NIV is required if the acidosis does not resolve quickly. ${ }^{143}$ This avoidable problem has occurred historically during the transfer to hospital, prior to measurement of ABGs or before a definitive diagnosis is known. Furthermore, ambulance staff are often not informed at present of a diagnosis of COPD and may not be aware of the presence of other high-risk conditions such as kyphoscoliosis or respiratory failure due to neuromuscular conditions. ${ }^{447}$ These patients should be issued with an oxygen alert card (example shown in figure 8 ) and a $24 \%$ or $28 \%$ Venturi mask based on previous blood gas results. The recommended oxygen saturation will be based on the clinical scenario for each individual patient but will usually be $88-92 \%$, occasionally $85-$ $88 \%$ or $85-90 \%$ based on previous blood gas results. Patients should be instructed to show this card to the ambulance crew and emergency department staff in order to avoid the use of high oxygen concentrations. This scheme can be successful. ${ }^{448}$ The ambulance service should be informed about which patients are issued with oxygen alert cards. The Association of Ambulance Chief Executives (AACE) JRCALC UK Ambulance Services Clinical Practice Guidelines 2016: (London, AACE, 2016) reflect this 2017 BTS Guideline for the use of oxygen in COPD. $^{12}$

Good practice points (see also section 8.12)

$\checkmark$ Patients with COPD (and other at-risk conditions) who have had an episode of hypercapnic respiratory failure should be issued with an oxygen alert card and with a $24 \%$ or $28 \%$ Venturi mask. They should be instructed to show the card to the ambulance crew and emergency department staff in the event of an exacerbation.

$\checkmark$ The content of the alert card should be specified by the physician in charge of the patient's care, based on previous blood gas results.

$\checkmark$ If a patient has an oxygen alert card, initial oxygen therapy should be based on the guidance on the card until the results of blood gases are available.

$\checkmark$ Oxygen alert cards with agreed content can be obtained via the BTS website.

$\checkmark$ The primary care team and ambulance service should also be informed by the responsible clinician that the patient has had an episode of hypercapnic respiratory failure and carries an oxygen alert card. The home address and ideal oxygen concentration or target saturation ranges of these patients can be flagged in the ambulance control systems and information disseminated to ambulance crews when required. 
Figure 8 Example of oxygen alert card.

\section{OXYGEN ALERT CARD}

Name:

I have a chronic respiratory condition and I am at risk of having a raised carbon dioxide

level in my blood during flare-ups of my condition (exacerbations)

\author{
Please use my ___ $\%$ Venturi mask to achieve an oxygen saturation of \\ $\%$ to \\ $\%$ during exacerbations of my condition
}

Use compressed air to drive nebulisers (with nasal oxygen a $2 \mathrm{l} / \mathrm{min}$ )

If compressed air is not available, limit oxygen-driven nebulisers to 6 minutes $\checkmark$ Out-of-hours services providing emergency primary care services should be informed by a responsible clinician that the patient has had an episode of hypercapnic respiratory failure and carries an oxygen alert card. Use of oxygen in these patients will be guided by the instructions on the alert card or by a patient-specific protocol which can be shared by hospital teams, the ambulance service and the primary care team.

$\checkmark$ During treatment by ambulance staff, oxygen-driven nebulisers should be used for patients with asthma and may be used for patients with COPD in the absence of an air-driven compressor system. If oxygen is used for patients with known COPD, its use should be limited to $6 \mathrm{~min}$. This will deliver most of the nebulised drug dose but limit the risk of hypercapnic respiratory failure (section 10.8.2). Ambulance services are encouraged to explore the feasibility of introducing battery-powered, air-driven nebulisers or portable ultrasonic nebulisers.

$\checkmark$ If a patient is suspected to have hypercapnia or respiratory acidosis due to excessive oxygen therapy, the oxygen therapy should not be discontinued but should be stepped down to the lowest level required to maintain a saturation range of $88-92 \%$. This may be achieved using $28 \%$ or $24 \%$ oxygen from a Venturi mask or $1-2 \mathrm{~L} / \mathrm{min}$ via nasal cannulae depending on oxygen saturation and subsequent blood gas results.

\subsection{Choice of devices in prehospital care}

The range of oxygen delivery devices is very wide as discussed in section 10. However, most patients can be managed with one of five types of oxygen delivery device.

Good practice points

It is recommended that the following delivery devices should be available in prehospital settings where oxygen is administered (see section 10):

$\checkmark$ High-concentration reservoir mask (non-rebreathe mask) for high-concentration oxygen therapy;

$\checkmark$ Nasal cannulae (preferably) or a simple face mask for medium-concentration oxygen therapy;

$\checkmark$ Twenty-eight per cent Venturi mask for patients with definite or likely COPD (patients who have an oxygen alert card may have their own $24 \%$ or $28 \%$ Venturi mask);

$\checkmark$ Tracheostomy masks for patients with tracheostomy or previous laryngectomy.

\subsection{Emergency use of oxygen in patient's home}

The emergency use of oxygen in the patient's home will be determined by what is available in the patient's home (see section 10.4) or what is carried by the first responder. GPs are probably unlikely to carry an emergency oxygen supply such as a portable cylinder unless they work in remote or rural areas. If oxygen is available in the patient's home by either oxygen concentrator or short burst oxygen by cylinder, the flow rate should be guided by the use of pulse oximetry if available. Otherwise, the need for oxygen may need to be determined by the presence of central cyanosis which can be very difficult in poor light. Enough oxygen should be given to achieve $88-92 \%$ if the patient is known to be at risk of hypercapnia, or the target saturation as stated on their oxygen alert card. Otherwise flow rate should be titrated to achieve saturations of $94-98 \%$ or correction of cyanosis if no pulse oximeter is available. It is not recommended that patients adjust their own flow rate of an existing oxygen supply based on saturations or increase in breathlessness unless it is part of an agreed escalation plan.

Good practice points

$\checkmark$ GPs or first responders visiting a patient's home should carry a portable pulse oximeter to assess hypoxaemia and guide use of oxygen if available and should call emergency services if hypoxaemia or other serious illness is suspected.

$\checkmark$ Those attending patients as an emergency in rural or remote areas should consider carrying a portable oxygen cylinder as part of their emergency equipment.

\subsection{Use of oxygen by rescue services and other non-NHS first responders}

The BTS Guideline was written for use by healthcare professionals, mostly operating within the NHS and emphasises the strategy of titrating supplemental oxygen to target levels of oxygen saturation, facilitated by the use of pulse oximeters. ${ }^{2}$ However, it is recognised that emergency oxygen is used in many other circumstances, especially by volunteers involved in Mountain Rescue, Lifeboat Rescue, Cave Rescue and similar services. In these circumstances, training of volunteers is the responsibility of the individual organisation and it is recognised that oximeters may not be available in all circumstances where oxygen is used by such teams. Furthermore, there are many circumstances such as hypothermia and shock where oximetry readings may be unreliable and these circumstances are more likely to be present during call-outs by the above services than during general ambulance operations in the UK. The AACE JRCALC UK Ambulance Services Clinical Practice Guidelines 2016: London, AACE, 2016 contains succinct guidance for ambulance staff in the UK based on the BTS Guideline. ${ }^{12}$ It is recommended that other first responder organisations may wish to base their oxygen strategies on the principles of the BTS guideline and the succinct practical advice, tables and flowchart contained in the JRCALC drugs section, accepting the limitations previously listed and the challenging environment in which these teams may operate. It is appreciated that oxygen 
may be used on a more pragmatic basis if it is not possible to obtain a reliable oximetry reading due to conditions such as shock or hypothermia or if oximetry is not available. Organisations are advised to prepare guidance for situations when oximetry is unreliable or unavailable for the above reasons. Organisational guidance should reflect the general principles of the BTS and JRCALC guidelines but BTS cannot endorse the specific recommendations of other bodies, especially if oxygen is given without monitoring by pulse oximetry. It is recognised that voluntary organisations may have difficulty in providing training (and regular refresher training) in the use of oxygen and oximeters to large numbers of volunteers. However, BTS regards the use of oximetry to guide oxygen therapy as best practice. Organisations should give very careful consideration to any decision resulting in a pulse oximetry not being used at the casualty site and/or in transport of seriously ill patients based on purely financial reasons.

\subsection{Use of nitrous oxide/oxygen mixtures (eg, Entonox)}

Entonox is a 50:50 mixture of oxygen and nitrous oxide that has been widely used as an inhalational analgesic agent for many years. However, a Danish review in 1994 reported as follows: "There are no controlled studies concerning the effect of pain treatment in the pre-hospital phase. The few available controlled studies conducted inside the hospital have not shown significant pain-relieving effects of nitrous oxide for patients suffering from pain of acute medical or surgical origin when compared to other methods of pain treatment." ${ }^{449} \mathrm{Su}$ et $a l^{450}$ reported better pain control and a lower caesarean section rate when $50 \%$ nitrous oxide was given to 658 women in labour compared with 642 women given $50 \%$ oxygen. The commonest side effect was dizziness which affected 39\% of those given nitrous oxide and none of the control participants. Because of its rapid onset of action and lack of serious side effects, it has been widely used in providing analgesia during childbirth and in trauma patients, in the emergency department and in the ambulance or scene of an accident. The gas mixture is usually selfadministered using a facemask with a demand valve that the patient holds to their face. The demand valve opens to allow gas to flow when it detects an inspiration. The analgesic onset is rapid, occurring within a few breaths. The main side effect is drowsiness and if the patient takes too much, because they are holding the mask to their face, the mask will slip off and the patient returns to breathing ambient air again. The rapid washout of nitrous oxide means that the patient regains consciousness very quickly. The mixture is less dense than air and may expand in air filled cavities, and as such, is contraindicated in patients with known emphysema. ${ }^{451}$

There is a concern of course that if Entonox is used in patients who are at risk of hypercapnic respiratory failure, the high concentration of oxygen may precipitate hypercapnia. Again, if the patient does become drowsy because of hypercapnia or the sedating effect of Entonox, they may release the mask and return to breathing air again. Unfortunately, if the patient has a cause for hypoxaemia, this will result in swinging from hyperoxaemia to hypoxaemia. Thus for this reason, the guideline group advise that Entonox is best avoided in patients who are at risk of hypercapnia or hypoxaemia.

Evidence statement

- The use of Entonox gas mixture is based on expert opinion in the absence of randomised trials to study the effects on oxygen physiology. The value of Entonox as an analgesic agent is outside the scope of this guideline (evidence level 4).

\section{Recommendation}

M1: The use of Entonox gas mixture for analgesia should be avoided if possible in patients at risk of hypercapnic respiratory failure (grade D).

\subsection{Use of oxygen in prehospital care for pregnant women}

The use of oxygen in the management of pregnant women in prehospital care should follow the same principles as in hospital care (section 8.14). All healthcare professionals caring for women in pregnancy should be aware of the importance of left lateral tilt or manual displacement of the uterus in circumstances of trauma, collapse, etc. This applies at gestations above 20 weeks (ie, when the fundus of the uterus reaches the level of the umbilicus). This will potentially improve maternal cardiac output and improve the mechanics of breathing. Pregnant women suffering major trauma or severe hypoxaemia should be started on high-concentration oxygen via a non-rebreathing reservoir mask and those with milder hypoxaemia can use nasal cannulae or a simple face mask or Venturi mask to achieve an oxygen saturation of $94-98 \%$ in most cases.

If mild/moderate analgesia is required during transportation, 'Entonox' may be administered—see previous section 9.11.

Recommendations for oxygen use in pregnancy are given in section 8.14.

\section{SECTION 10: PRACTICAL ASPECTS OF OXYGEN THERAPY}

Oxygen delivery systems can be considered as two components: the method of storage and provision of oxygen (eg, cylinders); the method of delivery to the patient (eg, Venturi mask). The options available for both will depend on the environment in which it is being used and the needs of the patient.

\subsection{Oxygen storage and provision and safety}

\subsubsection{Cylinders (compressed gas)}

Cylinders contain compressed gas held under a very high pressure. They come in an array of sizes and hence capacity, ranging from small portable cylinders for individual patient use to large cylinders suitable for hospital use (table 12). These can be used for bedside administration where piped oxygen is not available or can be the supply for a piped system.

With recent changes in technology, high-pressure cylinders are now available (ie, filled to $200 \mathrm{bar}$ rather than $137 \mathrm{bar}$ which can contain $54 \%$ more gas for the same size cylinder). It is important for all users of oxygen to be aware that most oxygen cylinders are colour-coded (black cylinder with white shoulder) but some high-pressure oxygen cylinders are all white. Small lightweight cylinders are also available for ambulatory use (eg, some weigh $3.2 \mathrm{~kg}$ when full). All systems containing compressed gases in the UK are subject to the Pressure Systems Safety Regulations 2000 (SI 2000 No 128). These regulations are intended to prevent the risk of injury from pressurised systems. Oxygen supports combustion and there is a risk of fire if oxygen is used close to combustible materials and a source of ignition. There is one case report of a serious fire caused by spontaneous sparking in the outflow system of an oxygen cylinder. ${ }^{452}$ Although oxygen is not an explosive gas, it is possible

Table 12 Examples of oxygen cylinder sizes and capacities

\begin{tabular}{lllllllll}
\hline Size & C & CD & D & E & F & G & HX & J \\
\hline Height & 36 & 49 & 46 & 79 & 86 & 124 & 94 & 145 \\
Capacity (L) & 170 & 460 & 340 & 680 & 1360 & 3400 & 2300 & 6800 \\
\hline
\end{tabular}


for oxygen cylinders to explode if heated during a fire, and deaths have been recorded in these circumstances. ${ }^{453} 454$

Healthcare organisations must ensure that they have a policy in place which ensures the safety of patients, staff and contractors in the provision, storage, use and maintenance of compressed gas systems as required by the Health and Safety at Work Act 1974.

Clinicians using oxygen cylinders should check the labelling of the cylinder to ensure that it is an oxygen cylinder and checks should be made to ensure that the cylinder is not empty or near empty.

\subsubsection{Liquid oxygen}

Liquid oxygen is contained in pressure tanks and is obtained from atmospheric oxygen by fractional distillation. It has to be evaporated into a gas before use. Large tanks are often used by hospitals and small tanks can be used domestically. Portable liquid oxygen is also available in small portable containers which can be filled from the larger tanks.

\subsubsection{Oxygen concentrators}

Oxygen concentrators are largely used in the domiciliary setting for the provision of long-term oxygen therapy and are therefore not used in the acute setting so will not be covered further.

\subsection{Patient delivery methods/interfaces}

10.2.1 High-concentration reservoir mask (non-rebreathing mask)

This type of mask delivers oxygen at concentrations between $60 \%$ and $90 \%$ when used at a flow rate of $15 \mathrm{~L} / \mathrm{min}$ (figure 9). ${ }^{455}$ The delivered oxygen concentration is variable and will depend on the mask fit and the patient's breathing pattern. These masks are most suitable for trauma and emergency use in patients in whom carbon dioxide retention is unlikely.

\subsubsection{Simple face mask}

This type of mask delivers oxygen concentrations between $40 \%$ and $60 \%$ (figure 10). It is sometimes referred to as an MC Mask,

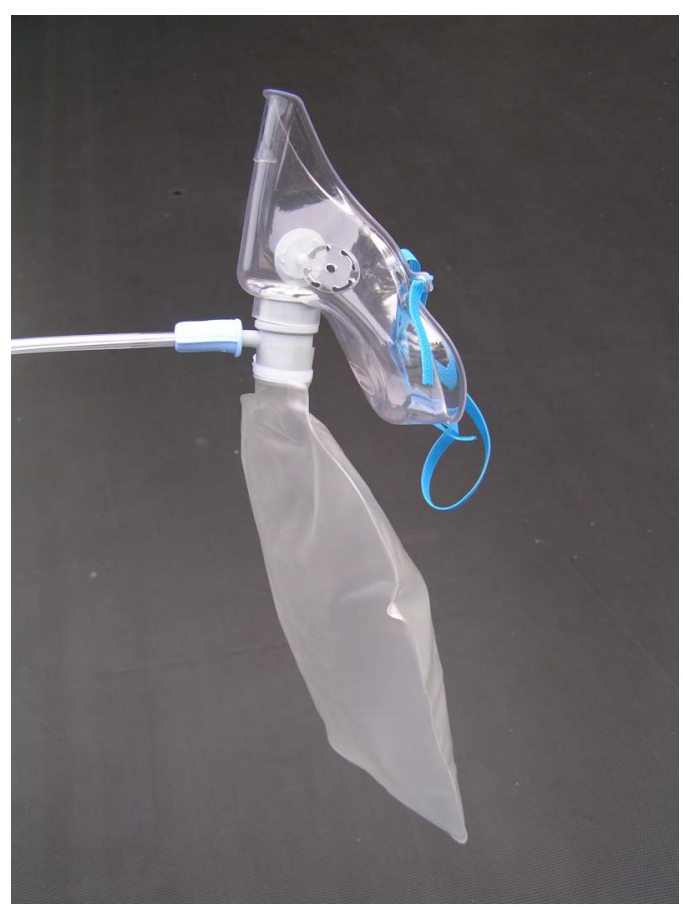

Figure 9 High-concentration reservoir mask (non-rebreathing mask).

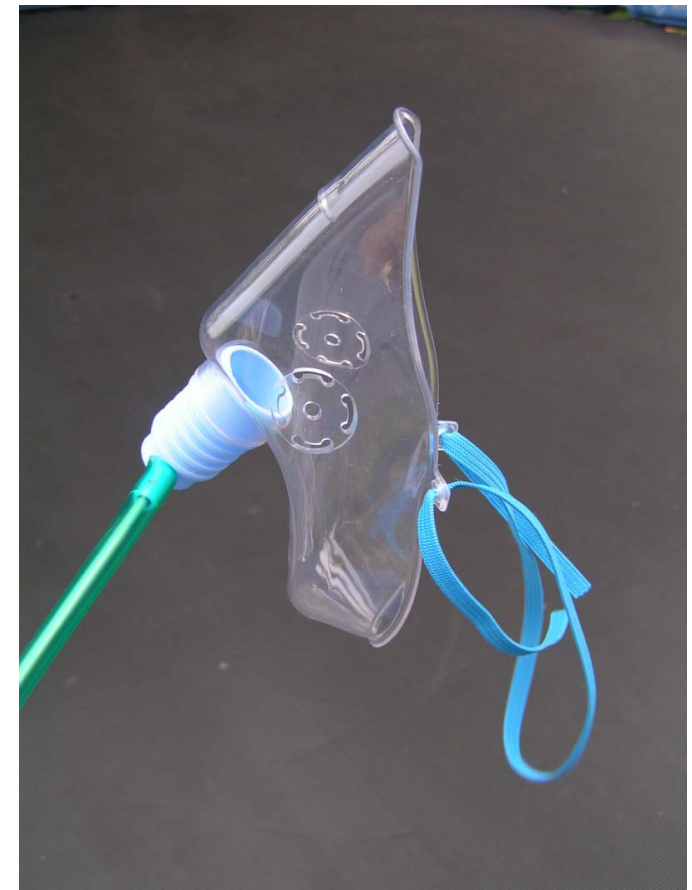

Figure 10 Simple face mask.

Medium Concentration Mask, Mary Catterall Mask or as a 'Hudson Mask', but the latter description is discouraged because the Hudson Company make many types of mask (including highconcentration reservoir masks). The guideline group favours the term 'simple face mask'. The oxygen supplied to the patient will be of variable concentration depending on the flow of oxygen and the patient's breathing pattern. The concentration can be changed by increasing or decreasing the oxygen flows between 5 and $10 \mathrm{~L} / \mathrm{min}$. However, different brands of simple face mask can deliver a different oxygen concentration at a given flow rate. Flows of $<5 \mathrm{~L} / \mathrm{min}$ can cause increased resistance to breathing, and there is a possibility of a build-up of carbon dioxide within the mask and rebreathing may occur. ${ }^{115}$

This mask is suitable for patients with respiratory failure without hypercapnia (type 1 respiratory failure) but is not suitable for patients with hypercapnic (type 2) respiratory failure. The mask may deliver a high concentration of oxygen $(>50 \%)$ and is therefore not recommended for patients who require lowconcentration oxygen therapy because of the risk of carbon dioxide retention. Patients using a simple face mask may have an inspiratory flow rate greater than the gas flow rate from the mask, so the simple face mask should not be used at flow rates below $5 \mathrm{~L} / \mathrm{min} .{ }^{115}$ Several publications have shown that patients who require medium-concentration oxygen therapy tend to prefer nasal cannulae to simple face masks and the cannulae are more likely to be left in position by the patient and less likely to fall off. ${ }^{456-459}$

\subsubsection{Venturi mask}

A Venturi mask will give an accurate concentration of oxygen to the patient regardless of oxygen flow rate (the minimum suggested flow rate is written on each Venturi device and the available options are shown in table 13; figure 11A,B). The oxygen concentration remains constant because of the Venturi principle. The gas flow into the mask is diluted with air which is entrained via the cage on the Venturi adaptor. The amount of air sucked 
Table 13 Total gas flow rate from Venturi masks at different oxygen flow rates

\begin{tabular}{lllllll}
\hline \multicolumn{7}{l}{ Venturi values } \\
\cline { 2 - 7 } Oxygen flow L/min & $\mathbf{2 4}$ & $\mathbf{2 8}$ & $\mathbf{3 1}$ & $\mathbf{3 5}$ & $\mathbf{4 0}$ & $\mathbf{6 0}$ \\
\hline 20 & & & & & 84 & 40 \\
15 & & & & 84 & 82 & 30 \\
12 & & & & 67 & 50 & 24 \\
10 & & & 78 & 56 & 41 & \\
8 & & 89 & 63 & 46 & & \\
6 & & 67 & 47 & & & \\
4 & 102 & 44 & & & & \\
2 & 51 & & & & &
\end{tabular}

into the cage is related to the flow of oxygen into the Venturi system. The higher the flow the more air is sucked in. The proportions remain the same and therefore the Venturi mask delivers the same concentration of oxygen as the flow rate is increased.

Venturi masks are available in the following concentrations: $24 \%, 28 \%, 31 \%, 35 \%, 40 \%$ and $60 \%$. They are suitable for all patients needing a known concentration of oxygen, but $24 \%$ and $28 \%$ Venturi masks are particularly suited to those at risk of carbon dioxide retention. A further benefit of Venturi masks is that the flow rate of gas from the mask will usually exceed the inspiratory flow rate of the patient. One study has shown that patients with a respiratory rate $>30$ breaths/min often have an inspiratory flow rate above the minimum flow rate specified on the mask packaging. ${ }^{303}$ Therefore, for patients with a high respiratory rate, it is suggested that the flow rate for Venturi masks should be set above the minimum flow rate listed on the packaging (increasing the oxygen flow rate into a Venturi mask does not increase the concentration of oxygen which is delivered). The accuracy of oxygen delivery from a Venturi mask is greatly reduced if the mask is not accurately placed on the patient's face. ${ }^{460}$

Patients with a respiratory rate $>30$ breaths/min often have a flow rate which is above the minimum delivered by the Venturi system as specified by the flow rate recommended for the mask. The flow may be increased as shown in figure 11B.

Venturi masks deliver a constant percentage of oxygen but the effect on the patient will depend on the condition being treated and on the breathing pattern and baseline oxygen saturation of the patient. As might be expected from the oxygen dissociation curve, patients with an oxygen saturation that is already in the normal range will have a very small rise in oxygen saturation (although the $\mathrm{PaO}_{2}$ is likely to rise substantially). However, patients with very low oxygen saturation will have a marked rise if given even a small concentration of oxygen. This is because the oxygen dissociation curve is actually a 'rapid escalator' rather than a 'slippery slope'. ${ }^{102} 106$ This is illustrated in figure 12 which demonstrates that a small increase in inspired oxygen concentration can make a big difference when the
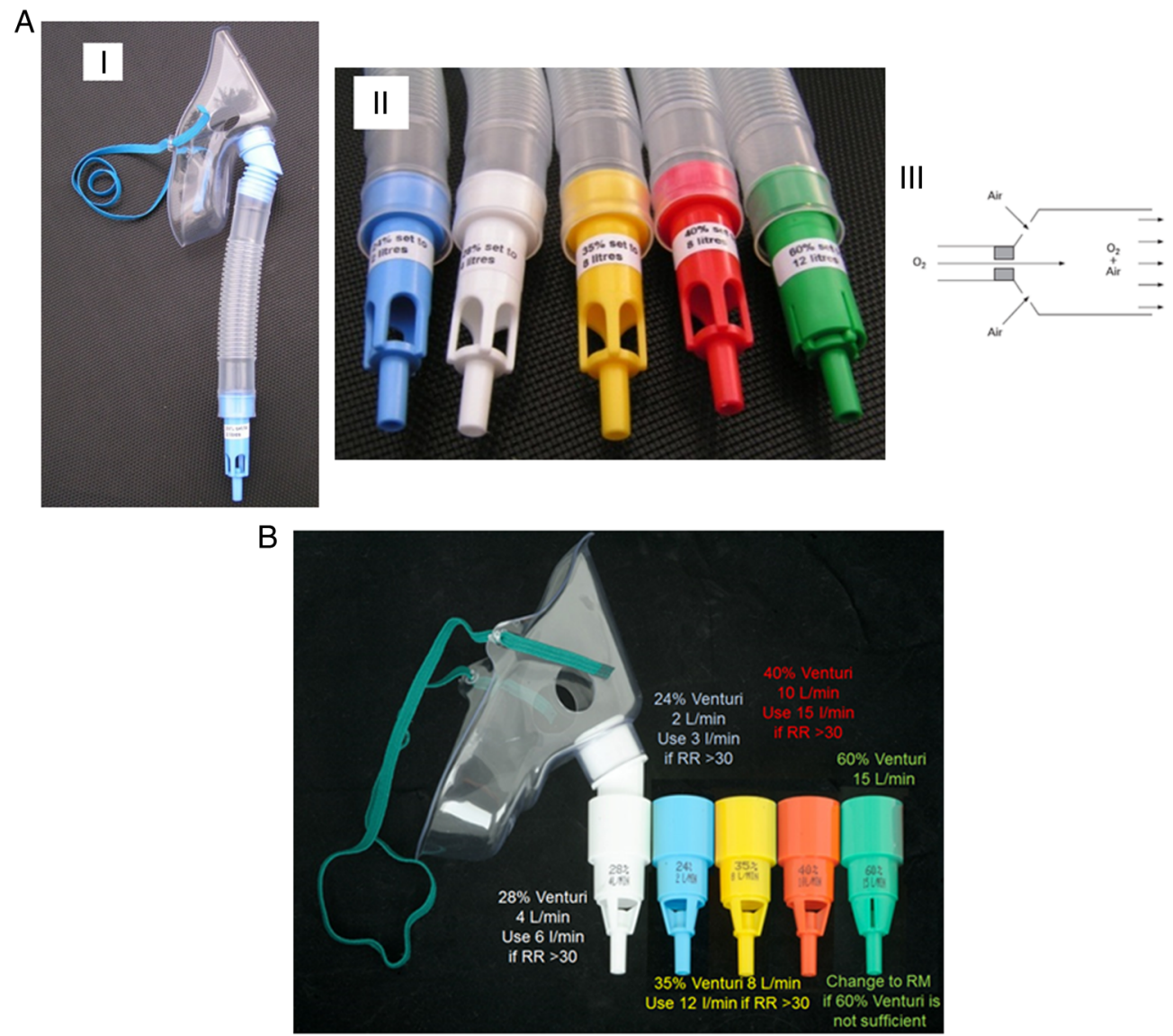

Figure 11 (A) (a) Venturi mask, (b) range of concentrations available, (c) operation of Venturi valve. For 24\% Venturi mask the typical oxygen flow of $2 \mathrm{~L} / \mathrm{min}$ gives a total gas flow of $51 \mathrm{~L} / \mathrm{min}$. For $28 \%$ Venturi mask, $4 \mathrm{~L} / \mathrm{min}$ oxygen flow gives total gas flow of $44 \mathrm{~L} / \mathrm{min}$ (table 13 ). (B) Suggested flow rates for Venturi masks and adjustment for high respiratory rate. RM, reservoir mask; RR, relative risk. 


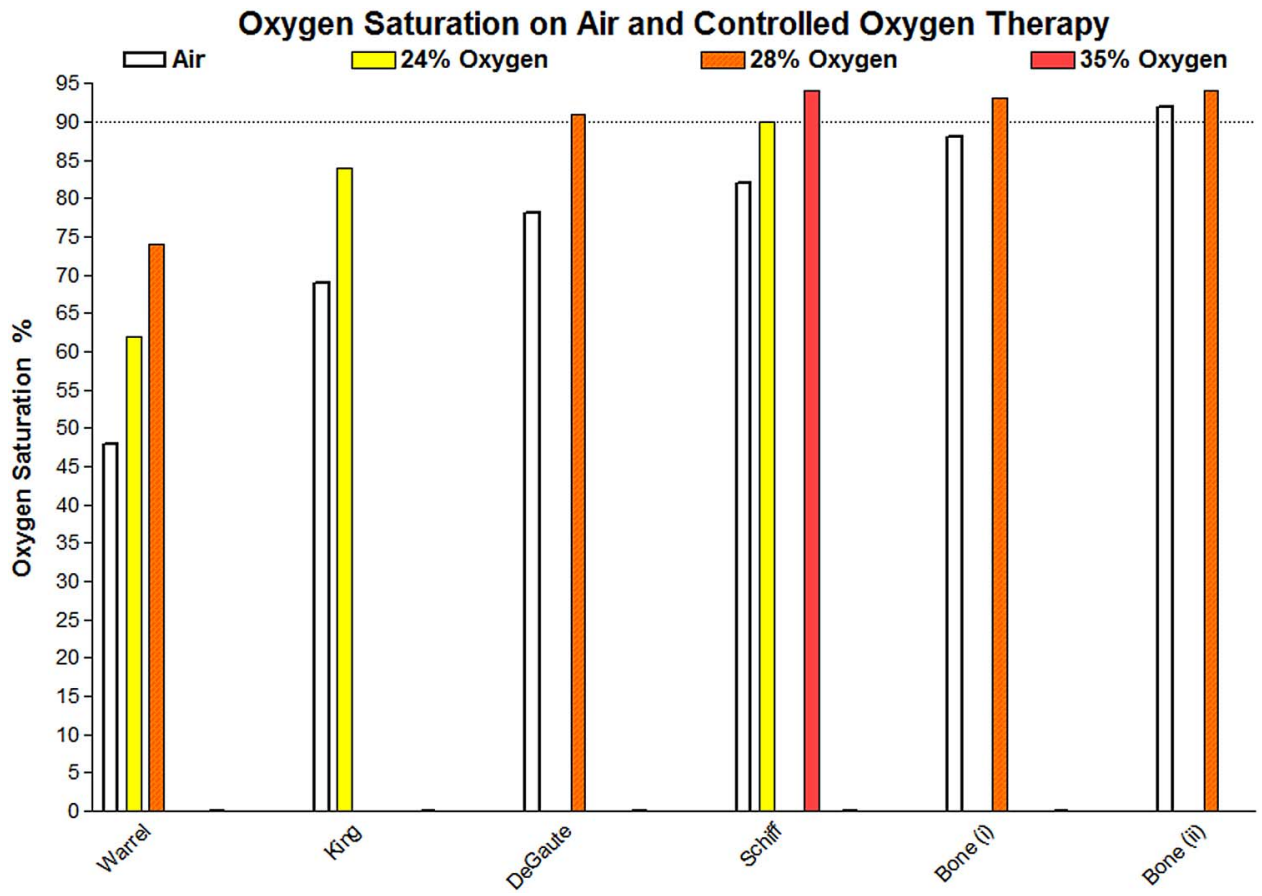

Figure 12 Oxygen saturation response to treatment with $24 \%, 28 \%$ and $35 \%$ oxygen in hypoxaemic patients with COPD. This illustration shows actual oxygen saturations from Warrel et $a^{85}$ and King et $a^{293}$ together with calculated saturations from DeGaute et al ${ }_{1}^{461}$ Schiff and Massaro $^{292}$ and Bone et al ${ }^{462}$ (two different groups of patients). COPD, chronic obstructive pulmonary disease.

saturation is below $80 \%$ but the response to oxygen is much less if the saturation is close to or above $90 \%$.

\subsubsection{Nasal cannulae}

Nasal cannulae can be used to deliver low-concentration and medium-concentration oxygen concentrations (figure 13). However, there is wide variation in patients' breathing patterns so the same flow rate of nasal oxygen may have widely different effects on the blood oxygen and carbon dioxide levels of different patients. Nasal cannulae at $1-4 \mathrm{~L} / \mathrm{min}$ can have effects on oxygen saturation approximately equivalent to those seen with $24-40 \%$ oxygen from Venturi masks. ${ }^{230} 463$ The actual concentration of oxygen delivered $\left(\mathrm{FiO}_{2}\right)$ cannot be predicted and so cannot be used if a calculation of A-a gradient is required. The oxygen concentration continues to rise up to flows above $6 \mathrm{~L} / \mathrm{min}$. Some patients may experience discomfort and nasal dryness at flows above $4 \mathrm{~L} / \mathrm{min}$, but they can be well tolerated (see section 10.2.5). ${ }^{230}{ }^{464}$ Although one might expect mouth breathing to reduce the efficiency of nasal cannulae, the majority of studies have shown that mouth breathing results in either the same inspired oxygen concentration or a higher concentration, especially when the respiratory rate is increased. ${ }^{464}$ This is important because patients with acute breathlessness are likely to breathe quickly and via the mouth rather than the nose. As there is marked individual variation in breathing pattern, the flow rate must be adjusted based on oximetry measurements and, where necessary, blood gas measurements. A cross-over comparison of nasal cannulae versus a Venturi mask (both adjusted to give satisfactory initial oxygen saturation) showed that the oxygen saturation of patients with exacerbated COPD fell below $90 \%$ for 5.4 hours/day during treatment with nasal cannulae compared with 3.7 hours/day during treatment with a Venturi mask. ${ }^{465}$ However, that study was published in 1999 before oximetery was widely used to monitor oxygen saturation.

The upper range of oxygen delivery from nasal cannulae is a little lower than the output of a simple face mask, but the lower range goes a lot lower than a simple face mask which should not be used below a flow rate of $5 \mathrm{~L} / \mathrm{min}$ (about $40 \%$ oxygen). ${ }^{115}$ The performance and variation of nasal cannulae for medium-concentration oxygen therapy is broadly similar to that of the simple face mask, both in laboratory experiments $^{230} 464$ and in clinical practice. ${ }^{457-459} 466467$ One study suggested that the saturation was lower with nasal cannulae than with simple face masks in a subgroup of men following abdominal surgery. ${ }^{467}$ However, the use of target ranges would resolve this issue. Three patient preference studies comparing nasal cannulae with simple face masks in postoperative care found that patient preference was strongly in favour of nasal cannulae with up to $88 \%$ of patients preferring cannulae to masks. ${ }^{457-459}$ Another advantage of cannulae over simple face masks is that they are less likely to be removed accidentally and they allow the patient to speak and eat. ${ }^{458} 468$ There are no comparisons of these devices in acute care, but there is no reason to believe that the results would be any different for patients requiring medium-concentration oxygen therapy. Nasal cannula (not Venturi masks) were used in the Austin et al's ${ }^{51}$ study which showed improved survival from controlled oxygen therapy in AECOPD.

Advantages of nasal cannulae compared with simple face masks for medium-concentration oxygen therapy

- Comfort (but a minority of patients dislike the flow of oxygen into the nose, especially above $4 \mathrm{~L} / \mathrm{min}$ ).

- Adjustable flow gives wide oxygen concentration range (flow rate of $1-6 \mathrm{~L} / \mathrm{min}$ gives $\mathrm{FiO}_{2}$ from $\sim 24 \%$ to $~ 50 \%$ ), suitable for variable oxygen therapy and concentration titration.

- Patient preference.

- No claustrophobic sensation.

- Not taken off to eat or speak and less likely to fall off.

- Less affected by movement of face.

- Less inspiratory resistance than simple face masks.

- No risk of rebreathing of carbon dioxide.

- Cheaper. 
Figure 13 Nasal cannulae.
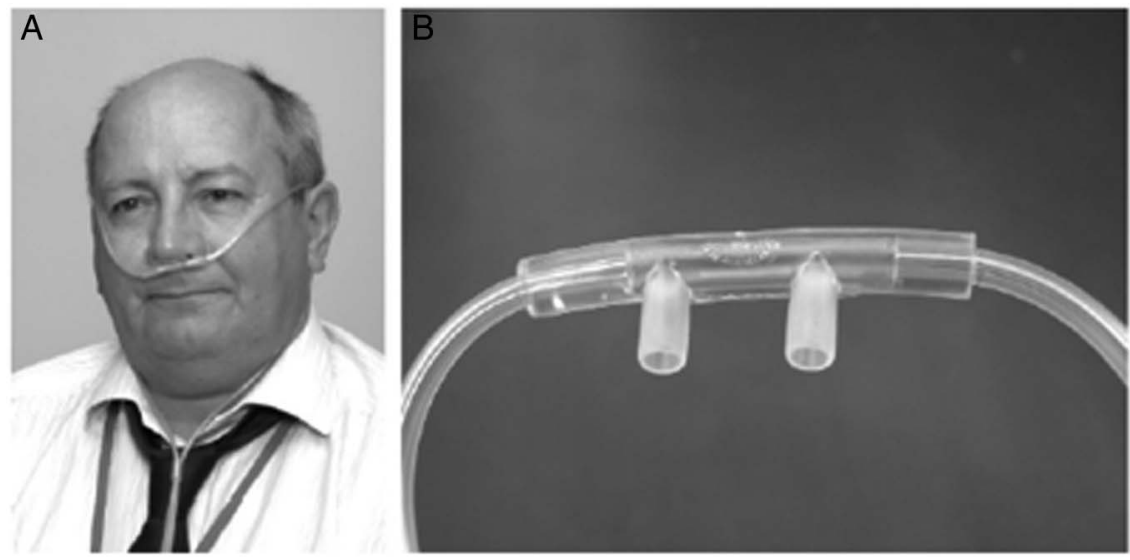

Disadvantages of nasal cannulae

- May cause nasal irritation or soreness.

- May not work if nose is severely congested or blocked.

- Actual concentration of oxygen $\left(\mathrm{FiO}_{2}\right)$ cannot be predicted

10.2.5 High-flow humidified oxygen via nasal cannulae

There is increasing use of HFNC as an alternative delivery device for adults requiring medium-concentration and highconcentration oxygen therapy (figure 14). There is currently a small body of evidence to support its use as well as growing interest from positive clinical observations. The therapeutic effects are thought to be multifactorial and include delivery of increased $\mathrm{FiO}_{2}$, a CPAP effect and greater comfort for the patient when compared with face masks. To deliver HFNC, three elements are required: a patient interface, a gas delivery device and a humidifier. However, specific research on the effect of humidification and high-flow oxygen therapy is lacking (see section 10.6.1). A flow of at least $60 \mathrm{~L} / \mathrm{min}$ can be accommodated via standard dimension prongs on adult nasal cannula. Several manufacturers provide these, specifically designed for high-flow application. Ritchie et $a l^{469}$ reported a positive airway pressure of about $7 \mathrm{~cm} \mathrm{H}_{2} \mathrm{O}$ in normal volunteers at a flow of $50 \mathrm{~L} / \mathrm{min}$. Trials to date are few and small; however, Tiruvoipati et $a l^{470}$ performed a prospective randomised trial of 50 patients postextubation, who required high-flow oxygen therapy. This study showed HFNC to be as effective as high-flow face mask in delivering oxygen to extubated patients as well as a significant difference in tolerance of HFNC and a trend towards improved comfort in this group. A recent study by Hernández et $a l^{471}$ randomised 527 mechanically ventilated patients at low risk of reintubation to either high-flow nasal oxygen or conventional oxygen for 24 hours after extubation.

They reported that the use of HFNC oxygen compared with conventional oxygen therapy reduced the risk of reintubation within 72 hours $(4.9 \%$ vs $12.2 \%$; $=0.04)$. A prospective sequential comparison trial by Roca et al ${ }^{472}$ examined 20 patients with acute hypoxaemic respiratory failure and compared HFNC with conventional oxygen therapy. HFNC was better tolerated and more comfortable than face mask. HFNC was associated with better oxygenation and lower respiratory rate. Cuquemelle et $a l^{473}$ also reported greater patient comfort using this system. In further support a study by Parke et al, ${ }^{474}$ 60 patients with mild-to-moderate hypoxemic respiratory failure were randomised to receive HFNC or high-flow face mask and they analysed the success of allocated therapy, NIV rate and oxygenation. Significantly, more HFNC patients succeeded with their allocated therapy and the rate of NIV in this group was
$10 \%$, compared with $30 \%$ in the high-flow face mask group. The HFNC patients also had significantly fewer desaturations.

Frat et $a l^{475}$ randomised 310 patients with acute hypoxaemic respiratory failure (without hypercapnia) to treatment with HFNC, reservoir mask or NIV and they found that HFNC increased the number of ventilator-free days and reduced 90-day mortality but did not significantly reduce the intubation rate which was the primary end point of the study.
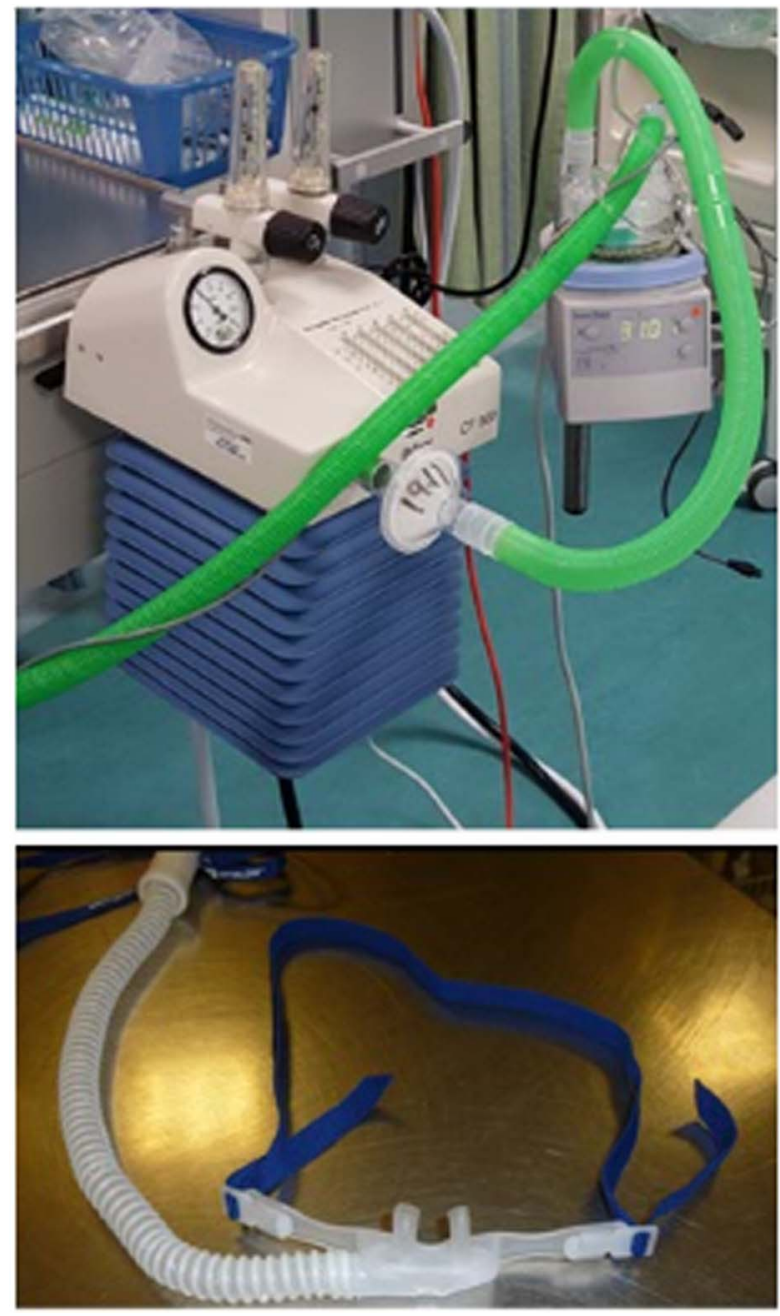

Figure 14 High-flow humidified nasal cannulae, flow generator and humidifier system. 
Larger and more comprehensive studies are still awaited but research to date is encouraging, indicating that HFNC may have an important role to play in the delivery of high-flow oxygen both in terms of physiological advantages and the tolerance and comfort of the individual patients who are not at risk of hypercapnia (evidence level 2- to 1-).

Good practice point

$\checkmark$ High-flow nasal oxygen should be considered as an alternative to reservoir mask treatment in patients with acute respiratory failure without hypercapnia.

\subsubsection{Tracheostomy mask}

These devices are designed to allow oxygen to be given via a tracheostomy tube or to patients with previous laryngectomy (ie, neck breathing patients; figure 15). The oxygen flow rate should be adjusted to achieve saturation in accordance with tables 1-4 and chart 1 (figure 1). Oxygen given in this way for prolonged periods needs constant humidification and patients may need suction to remove mucus from the airway.

\subsubsection{Non-invasive ventilation}

This treatment option is beyond the scope of the present guideline. Readers are referred to the BTS guideline concerning the use of NIV in patients with exacerbations of COPD. 209299

\subsection{Oxygen carriage and delivery during patient transport in ambulances}

Transport of oxygen cylinders in vehicles comes under the Transport of Dangerous Substances Act or the Carriage Regulations only if $1000 \mathrm{~L}$ or more (measured by the water capacity of the cylinder) is carried at any one time. Ambulances are therefore exempt from this. Normal health and safety requirements will still apply. ${ }^{476} 477$

\subsubsection{Health and Safety Executive guidance for safe use of} oxygen cylinders 476477

- All cylinders must be secured appropriately so they cannot move in transit (includes portable cylinders).

- No smoking in the vicinity of cylinders.

- Cylinders must be checked regularly for obvious signs of leakage.

- Cylinders must be kept out of direct sunlight.

- Green warning triangle 'compressed gas' should be displayed on the vehicle.

- Cylinders should never be lifted by the neck.

- They should only be changed by suitably trained personnel.

- Apart from portable cylinders, all cylinders should be moved using a cylinder trolley.

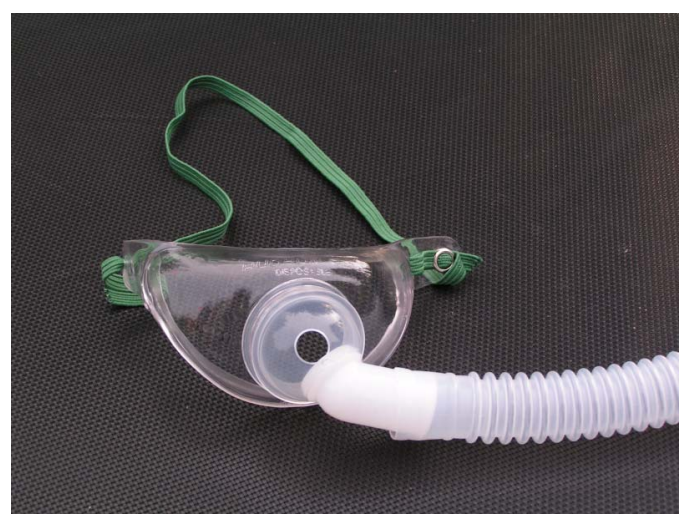

Figure 15 Tracheostomy mask.
- Cylinders must be turned off/fully closed when not in use

- Cylinders must be in date. Staff should check the expiry date prior to use.

\subsubsection{Oxygen use by UK ambulance services}

Currently, within the UK, the ambulance service-whether NHS or private-has a range of vehicles and oxygen delivery systems at their disposal. There is an increasing use of cycle response units which tend to use the lightweight $\mathrm{AZ}$ or $\mathrm{C}$ sized cylinder with a capacity of $170 \mathrm{~L}$. Motorcycle response units are generally equipped with the same AZ or $\mathrm{C}$ sized cylinders. Fast response units based on cars tend to be equipped with at least two of the lightweight CD sized cylinders which hold $460 \mathrm{~L}$. The CD cylinder is also the size favoured by mountain, cave and mines rescue teams.

Front-line ambulances are usually equipped with piped oxygen fittings (Schraeder type) and supplied from two HX sized $(2300 \mathrm{~L})$ cylinders, as well as carrying at least two CD sized cylinders to power a portable oxygen-powered resuscitator. The piped supply has several outlet points placed in strategic positions to which are attached standard Schrader flow meters (0-15 L/min). This enables oxygen to be given throughout the patient's journey. The ambulance is also equipped with a portable supply which can be used at the site of an accident, taken into a patient's home or can be used when transferring a patient. They carry a range of patient interfaces for delivering the oxygen under the different circumstances encountered.

Portable resuscitators are always capable of supplying freeflow oxygen therapy as well as their resuscitator facility. Again, there are a variety of portable oxygen-powered resuscitators and it is beyond the scope of these guidelines to describe each one available for use in prehospital care. It is strongly suggested that those practitioners who need to work closely with the ambulance service should become familiar with the equipment used by their local ambulance service provider. With the possible exception of the cycle response units, all types of ambulance service response will have portable resuscitators, bag-valve mask devices and hand-operated suction as a minimum. Front-line emergency ambulances will also have rechargeable batterypowered suction available.

Patient transport service ambulances are equipped with an oxygen supply, normally an HX cylinder $(2300 \mathrm{~L})$ delivering the oxygen via a flow meter attached directly to the cylinder. Such vehicles also tend to carry basic hand-held suction devices. A range of oxygen masks are usually available for the different types of patients carried on such vehicles. Vehicles that are equipped with an oxygen supply should also carry oximeters to ensure appropriate use of oxygen (see section 9.8 for advice on which oxygen delivery devices should be carried in ambulances).

\subsection{0xygen carriage in other vehicles and in primary care settings and patients' homes}

10.4.1 Oxygen carriage in private cars (Health and Safety Executive guidance)

When travelling by car, patients have the freedom to carry their own portable oxygen cylinder. ${ }^{476} 477$ Some GPs in rural areas also carry oxygen in their cars. However, it is advised that certain safety precautions should be followed:

- Patients are allowed to carry oxygen cylinders for their own use without putting any labels or signs on their vehicle. This includes public transport such as buses or taxis. It is an offence to display a hazard diamond if oxygen is not being transported in a vehicle so it is preferable not to use a hazard triangle on private vehicles. 
- The cylinder should be secure within the car and cannot move during transport or in the event of an accident. The authors of this guideline are aware of one fatality caused by a patient smoking in a vehicle where an unsecured liquid oxygen cylinder spilt into the floor-well during sudden braking.

- Patients should inform their car insurance company if oxygen is carried in the car.

\subsubsection{Medical centres and primary care practices}

The majority of medical centres and practices should have a supply of oxygen for emergency use. Generally, cylinders with integral high-flow regulators should be ordered. Otherwise, the cylinder must be fitted with a high-flow regulator capable of delivering a flow of up to $15 \mathrm{~L} / \mathrm{min}$ in order to deliver mediumconcentration and high-concentration oxygen therapy. A recommended list of oxygen delivery devices for use in prehospital care is given in section 9.8 .

Emergency oxygen should be available in primary care medical centres, preferably using oxygen cylinders with integral high-flow regulators. Alternatively, oxygen cylinders fitted with high-flow regulators (delivering up to $15 \mathrm{~L} / \mathrm{min}$ ) must be used to allow use with reservoir masks (see recommendation V1).

\subsubsection{Emergency use of oxygen in the patient's home}

In patients' homes, oxygen is usually provided for long-term therapy with an oxygen concentrator and an ambulatory supply with lightweight cylinders (or a portable liquid oxygen system). Long-term oxygen therapy is covered in other guidelines. In some circumstances, there may be a supply of cylinders for short-term/short-burst therapy or palliative use. The existing home oxygen supply may be used by a patient or GP in an emergency situation before the arrival of an ambulance using the patient's existing interface. If a GP is attending a patient at home with oxygen, ideally, the use of oxygen should be guided by pulse oximetry. The existence of any oxygen alert card should be asked for so that the emergency services attending can be aware of the target saturation and oxygen supply titrated accordingly.

The patient/carers should be made aware of the following Health and Safety recommendations: ${ }^{476} 477$

- All cylinders should be stored on a cylinder trolley or suitably secured so they cannot be knocked over.

- There should be no trailing oxygen tubing.

- A green warning triangle for 'compressed gas' should be displayed by the front door (warns emergency services in the event of a fire).

- The minimum number of cylinders should be stored in the house.

- There should be no smoking in the vicinity of oxygen cylinders.

- Cylinders must be checked regularly for obvious signs of leakage.

- Cylinders must be kept out of direct sunlight.

- Oxygen must not be used near a naked flame or source of heat.

\subsection{Oxygen delivery systems in hospitals}

Most hospitals have piped oxygen systems as described previously, although some wards can still be found where piped oxygen is not available and large compressed gas cylinders are used to supply the oxygen. Acute hospitals can spend up to $£ 100000$ per annum on liquid oxygen, so any device that uses lower oxygen flow rates could have significant economic savings for hospitals (eg, nasal cannulae instead of a simple face mask for medium-concentration oxygen).

\subsubsection{Perioperative and postoperative care}

Medium-concentration masks and nasal cannulae are usually sufficient (target saturation 94-98\%) except for patients with known significant COPD who should receive oxygen from a $24 \%$ or $28 \%$ Venturi mask or $1-2 \mathrm{~L} / \mathrm{min}$ from nasal cannulae aiming at a saturation range of $88-92 \%$.

\subsubsection{Emergency departments}

Medium-concentration or high-concentration oxygen is normally used (via nasal cannulae, simple face mask or reservoir mask), but particular attention should be given to patients who have hypercapnic respiratory failure when a $24 \%$ or $28 \%$ Venturi mask or nasal cannulae at a flow rate of $1-2 \mathrm{~L} / \mathrm{min}$ would be appropriate.

\subsubsection{General wards and respiratory wards}

The method of oxygen delivery will depend on the following circumstances:

- Expected duration of treatment.

- Type of respiratory illness.

- Pattern of breathing (high or low respiratory rate and drive).

- Need for humidification.

- Risk of carbon dioxide retention.

- Presence of confusion and its effect on potential compliance.

Nasal cannulae, simple face masks, reservoir masks and Venturi masks should be used where appropriate (see tables 1-4 and charts 1 and 2 in the main guideline). Nasal cannulae at flow rates or $0.5-1.0 \mathrm{~L} / \mathrm{min}$ are sometimes used as a substitute for Venturi masks in acute or postacute patients with COPD on respiratory wards (adjusting flow as necessary to achieve the desired ABG tensions). This practice requires the use of paediatric flow meters to ensure consistent and finely calibrated oxygen delivery and is not recommended outside specialist units.

\subsubsection{Devices used in emergency oxygen therapy}

Based on the advantages of each delivery system discussed above, the following recommendations are made for delivery of oxygen in medical emergencies. It is likely that additional equipment will be maintained in specialist units, but specialist treatment is outside the scope of the present guideline.

Good practice points (see tables 1-4)

$\checkmark$ Most hospital patients can be managed with the same delivery device, as in good practice points which follow section 9.8, but a $24 \%$ Venturi mask should also be available.

- The high-concentration reservoir mask at $15 \mathrm{~L} / \mathrm{min}$ is the preferred means for delivering high-concentration oxygen to critically ill patients until reliable pulse oximetry monitoring has been established.

- Nasal cannulae should be used rather than simple face masks in most situations requiring mediumconcentration oxygen therapy. Nasal cannulae are preferred by patients for reasons of comfort and they are less likely to be removed during meals. There is also a cost-saving.

- The flow rate from nasal cannulae for mediumconcentration oxygen therapy should be adjusted between 2 and $6 \mathrm{~L} / \mathrm{min}$ to achieve the desired target saturation.

- Venturi masks are recommended for patients requiring precise control of $\mathrm{FiO}_{2}$. Venturi masks can deliver a constant $\mathrm{FiO}_{2}$ of $24 \%, 28 \%, 31 \%, 35 \%, 40 \%$ and $60 \%$ oxygen with a greater gas flow than a simple face mask. 
In those at risk of developing hypercapnic respiratory failure with oxygen therapy, the use of Venturi masks may reduce this risk. Furthermore, there is less likelihood of dilution of the oxygen stream by room air if the patient's inspiratory flow rate exceeds the flow rate delivered by the face mask.

$\checkmark$ For many patients, 24-28\% Venturi masks can be substituted with nasal cannulae at low-flow rates $(1-2 \mathrm{~L} / \mathrm{min})$ to achieve the same target range.

$\checkmark$ The flow rate from simple face masks should be adjusted between 5 and $10 \mathrm{~L} / \mathrm{min}$ to achieve the desired target saturation. Flow rates below $5 \mathrm{~L} / \mathrm{min}$ may cause carbon dioxide rebreathing and increased resistance to inspiration.

$\checkmark$ Patients with COPD with a respiratory rate of $>30$ breaths/ min should have the flow rate set to above the minimum flow rate specified for the Venturi mask and/or packaging (see figure 11B) (increasing the oxygen flow rate into a Venturi mask increases the total gas flow from the mask but does not increase the concentration of oxygen which is delivered).

$\checkmark$ HFNC are well tolerated and may be used as an alternative in hypoxic adult patients requiring medium-concentration to high-concentration oxygen therapy and who are not at risk of hypercapnia.

\subsubsection{Flow meters}

All oxygen delivery systems must have a method of taking the high pressure/flow of gas and reducing it so it can be administered to the patient at a specific flow depending on the individual's needs and mask being used. Piped oxygen points have Schrader flow meters and cylinders have pressure and flow regulators. Most oxygen flow meters use a floating ball to indicate the flow rate. The centre of the ball should be aligned with the appropriate flow rate marking. The example shown in figure 16 indicates the correct setting to deliver $2 \mathrm{~L} / \mathrm{min}$.

\subsubsection{Oxygen tubing and oxygen wall outlets}

Oxygen tubing is needed to connect flow meters and regulators to the patient delivery device. It is important to ensure that all tubing is connected correctly at both ends. The National Patient Safety Agency has reported frequent adverse events related to oxygen use, including four reports of instances where an oxygen mask was connected in error to a compressed air outlet instead of an oxygen outlet. Compressed air outlets are often used to drive nebulisers in hospitals because they are quieter than electrical compressors. However, the flow meter looks very similar to an oxygen flow meter and is often mounted beside an oxygen flow meter so it is very important to ensure that air flow meters are clearly labelled. There is a similar risk with other piped gas outlets such as those delivering nitrous oxide in some hospitals. Air flow meters are never required in an emergency and should be removed from wall sockets or covered by a designated 'hood' when not in use, an example is shown in figure 17.

The guideline authors are also aware of some cases where twin oxygen outlets were in use and the wrong one had been turned on or off. For example, one patient tried to turn off the oxygen flow after finishing a nebulised treatment but accidentally turned off the oxygen flow to a neighbouring patient with serious consequences. It is recommended that patients should not be allowed to adjust oxygen flow, especially if there are dual outlets.

Healthcare organisations should take measures to eliminate the risk of oxygen tubing being connected to the incorrect wall oxygen outlet or to outlets that deliver compressed air or other gases instead of oxygen. Air flow meters should be removed from the wall sockets or covered with a designated air outlet cover when not in use. Special care should be taken if twin oxygen outlets are in use (see recommendation V2).

Evidence statement

- Matters related to oxygen tubing and equipment and wall outlets are based on expert opinion and manufacturers' advice (evidence level 4).

\section{Recommendation}

V2: Healthcare organisations should take measures to eliminate the risk of oxygen tubing being connected to the incorrect wall oxygen outlet or to outlets that deliver compressed air or other gases instead of oxygen. Air flow meters should be removed from the wall sockets or covered with a designated air outlet cover when not in use. Special care should be taken if twin oxygen outlets are in use (grade D).

\subsection{Use of humidified oxygen}

\subsubsection{Rationale for use of humidified oxygen}

The upper airway normally warms, moistens and filters inspired gases. When these functions are impaired by a pathological process or when they are bypassed by an artificial airway, it is common practice to provide humidification. The main reason for using humidification, especially with high-flow oxygen, is
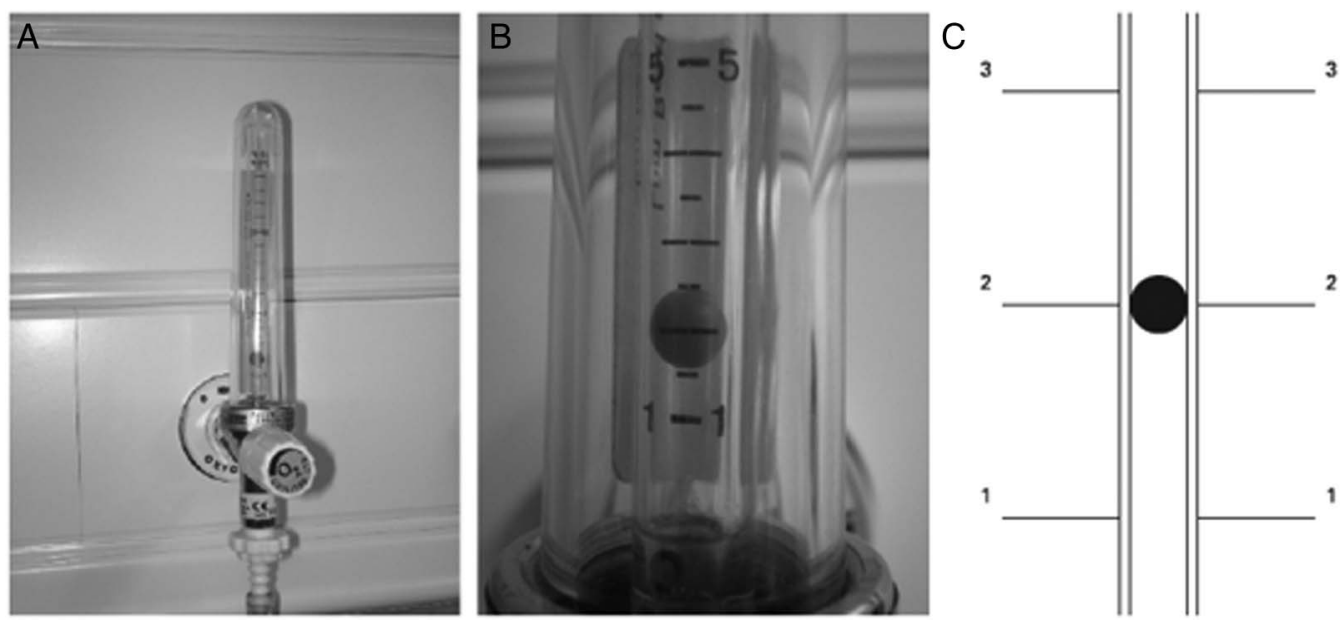

Figure 16 Flow meter showing correct setting to deliver oxygen at a rate of $2 \mathrm{~L} / \mathrm{min}$. 


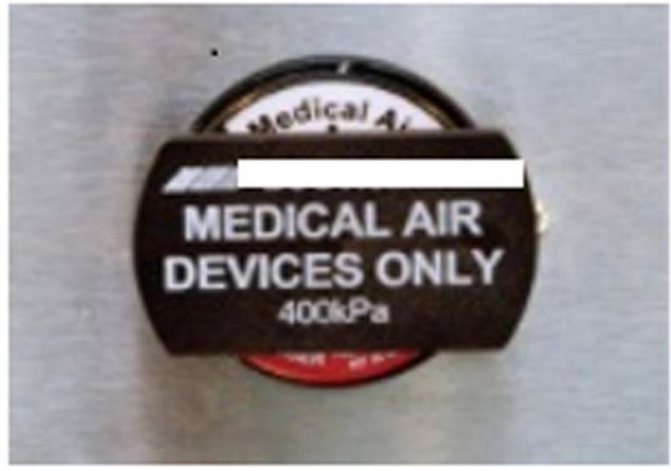

Figure 17 Air outlet cover.

that it may reduce the sensation of dryness in the upper airways that oxygen can cause. However, in the non-intubated population, there appears to be little scientific evidence of any benefit from humidified oxygen except that single doses of nebulised isotonic saline have been shown to assist sputum clearance and reduce breathlessness in patients with COPD. ${ }^{478} 479$ There is also evidence that humidification, when combined with physiotherapy, can increase sputum clearance in bronchiectasis. ${ }^{480}$ RCTs of the effects of humidified high-flow oxygen on patient comfort are required. Use of low-flow oxygen through nasal cannulae is well tolerated without the need for humidification. ${ }^{481}$ HFNC require warmed and humidified oxygen.

Evidence statement

- The use of humidified oxygen in acute care is based on expert opinion (evidence level 4).

Recommendations

Q1: Humidification is not required for the delivery of low-flow oxygen (mask or nasal cannulae) or for the short-term use of high-flow oxygen. It is not therefore required in prehospital care. Pending the results of clinical trials, it is reasonable to use humidified oxygen for patients who require high-flow oxygen systems for more than 24 hours or who report upper airway discomfort due to dryness (grade D).

Q2: In the emergency situation, humidified oxygen use can be confined to patients with tracheostomy or an artificial airway although these patients can be managed without humidification for short periods of time (eg, ambulance journeys) (grade D).

Q3: Humidification may also be of benefit to patients with viscous secretions causing difficulty with expectoration. This benefit can be achieved using nebulised normal saline (grade D).

\subsubsection{Use of bubble humidification systems}

Humidified oxygen is widely administered in hospitals across the UK and this is presumed to alleviate nasal and oral discomfort in the non-intubated patient. Humidification of supplemental oxygen was commonly delivered by bubbling oxygen through either cold or warm sterile water before it reached the patient. However, the effect on patient comfort is negligible. $^{481} 482$ Bubble humidifiers may, however, represent an infection hazard and should not be used. ${ }^{483}$ There is no evidence of a clinically significant benefit from 'bubble bottle' systems but there is an infection risk.

Evidence statement

- The advice not to use bubble bottles is based on expert opinion (evidence level 4).

Recommendation

Q4: Bubble bottles which allow a stream of oxygen to bubble through a container of water should not be used because there is no evidence of a clinically significant benefit but there is a risk of infection (grade D).

\subsubsection{Large volume nebulisation-based humidifiers}

If humidification is required, it should ideally deliver the inspired gas at a temperature of $32-36^{\circ} \mathrm{C}$. Cold water humidifiers are simple and inexpensive but less efficient than a warm water systems (about 50\% relative humidity at ambient temperatures). The warm water option is more effective, targeting a relative humidity of $100 \%$, but both systems are thought to be a potential infection control risk. Warm water humidifiers are expensive and mostly confined to ICUs and HDUs and thus outside the scope of this guideline.

Newer humidifying systems are really 'giant nebulisers' with a $1 \mathrm{~L}$ reservoir of saline or sterile water and an adjustable Venturi device (figure 18). These systems are attached directly to the oxygen flow meter and connected to an aerosol mask via Flex tube. They allow delivery of precise oxygen concentrations of $28 \%, 35 \%, 40 \%$ and $60 \%$ oxygen via their Venturi device. This requires a specific oxygen flow rate as well as adjusting the Venturi nozzle on the device. It is possible to deliver $24 \%$ oxygen using a special adaptor. These large volume humidifiers have a high humidification output. The main indication for use is to assist with expectoration of viscous sputum. There are no published randomised studies involving these devices, but it has been shown that single doses of nebulised saline can assist sputum production and relieve breathlessness in patients with COPD. 478479

\section{Good practice points related to humidified oxygen therapy}

$\checkmark$ Consider use of a large volume oxygen humidifier device for patients requiring high-flow rates or longer term oxygen, especially if sputum retention is a clinical problem.

$\checkmark$ In the absence of an artificial airway, the decision to humidify supplemental oxygen needs to be made on an individual basis but this practice is not evidence-based.

\subsection{Use of oxygen in patients with tracheostomy or laryngectomy}

The number of patients with a tracheostomy being cared for in a ward setting is increasing as critical care personnel use this as a method of facilitating weaning from mechanical ventilation. In the absence of a pressurised circuit, oxygen is predominantly delivered via tracheostomy mask. This is a variable performance device and delivers concentrations up to $60-70 \%$. If the patient deteriorates and requires an increased oxygen concentration exceeding the concentration that a variable performance interface can deliver (60-70\%), it will be necessary to seek an alternative delivery system, usually a T-piece device fitted directly to the tracheostomy tube.

With a mask system, the interface will be connected to a humidification system via elephant tubing. As inserting a tracheostomy tube bypasses the patient's natural mechanisms to warm and moisturise inspired gases, it is essential to humidify any supplemental oxygen being delivered to the tracheotomised patient. This will help maintain a patent tracheostomy tube, reducing the build-up of secretions within the inner tube or the tracheostomy itself and minimising any subjective discomfort that the patient may experience.

Evidence statement

- The use of tracheostomy masks is based on expert opinion and clinical experience (evidence level 4). 


\section{Recommendation}

P1: When oxygen is required by patients with prior tracheostomy or laryngectomy, a tracheostomy mask (varying the flow as necessary) should achieve the desired oxygen saturation (tables 1-4). An alternative delivery device, usually a T-piece device fitted directly to the tracheostomy tube, may be necessary if the patient deteriorates (grade D).

\subsection{Delivering oxygen to patients who require nebulised bronchodilator therapy \\ 10.8.1 Nebulised bronchodilator therapy in asthma}

In patients with acute severe asthma, oxygen should be used as the driving gas for the nebulised bronchodilators whenever possible at a gas flow rate of $6-8 \mathrm{~L} / \mathrm{min}$ because these patients are at risk of hypoxaemia. If the available cylinders in general practice do not produce this flow rate, an air-driven nebuliser (with electrical compressor) should be used with supplemental oxygen by nasal cannulae at 2-6 L/min to maintain an appropriate oxygen saturation level. There is some evidence that bronchodilator therapy (whether delivered by nebuliser or by metered dose inhaler) may cause pulmonary vasodilation leading to increased V/Q mismatch and reduced blood oxygen levels in acutely ill patients and in patients with stable COPD and asthma. ${ }^{484-486}$ The lowest oxygen saturation occurs not during bronchodilator therapy but about 25 min later. ${ }^{486}$ One group reported a rise in oxygen saturation during bronchodilator therapy. ${ }^{487}$

There is some evidence that bronchodilator therapy, however given, can cause increased V/Q mismatch and reduced blood oxygen levels in acutely ill patients shortly after treatment.

\subsubsection{Nebulised bronchodilator therapy for patients with COPD} and other risk factors for hypercapnic respiratory failure When an oxygen-driven nebuliser is given to patients with COPD there is a risk of hypercapnia and acidosis due to the high $\mathrm{FiO}_{2}$ which is delivered. In AECOPD the carbon dioxide level

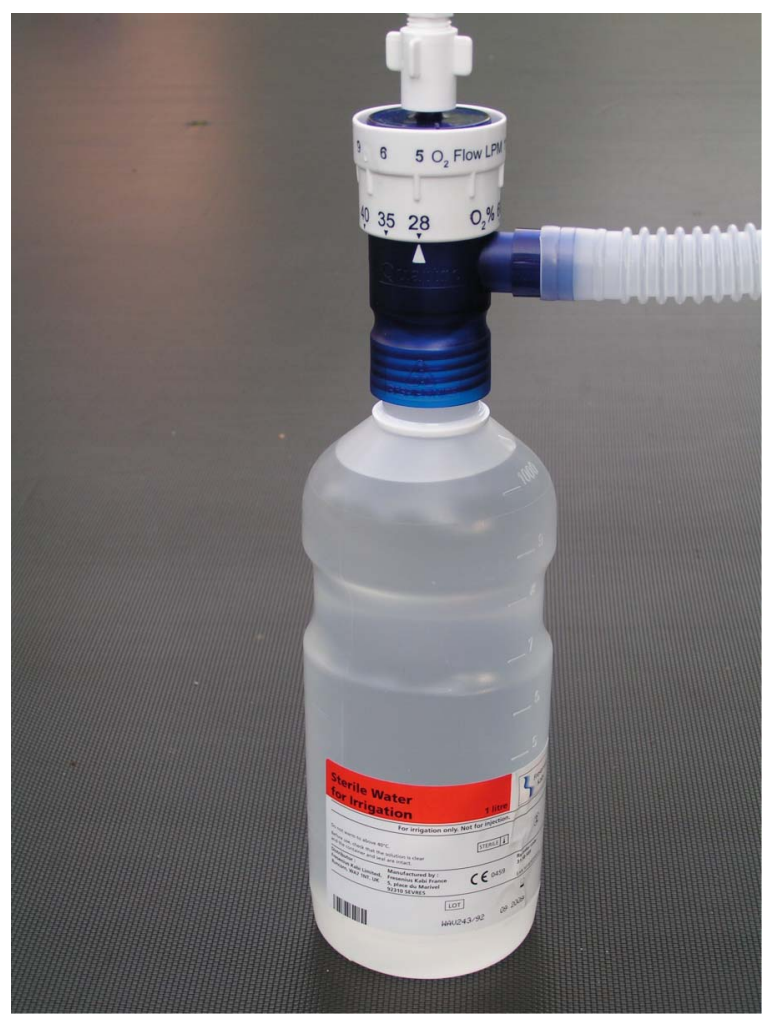

Figure 18 Large volume nebulisation-based humidifier. can rise substantially within $15 \mathrm{~min}$ of starting highconcentration oxygen therapy. ${ }^{48}$ Edwards et al ${ }^{488}$ have confirmed that oxygen-driven nebulisers cause elevation of the blood $\mathrm{CO}_{2}$ level in patients with COPD. The Austin et al's ${ }^{51}$ study which demonstrated improved mortality with targeted oxygen therapy in patients with AECOPD used air-driven nebulisers to ensure that the target saturation was maintained. When nebulised bronchodilators are given to hypercapnic patients, they should ideally be given using an electrical compressor or ultrasonic nebuliser and, if necessary, supplementary oxygen should be given concurrently by using nasal cannulae at $1-4 \mathrm{~L} / \mathrm{min}$ to maintain an oxygen saturation of $88-92 \%$. For hypercapnic patients or those with a history of hypercapnia, oxygen saturation should be monitored continuously during nebulised treatments. If an electrical compressor or ultrasonic nebuliser is not available, compressed air can be used to drive the nebuliser but care should be taken to ensure that the nebuliser is not attached to an oxygen outlet (see section 10.8). Once the nebulised treatment is completed, targeted oxygen therapy should be reinstituted.

Compressed air is not routinely available for treatment by ambulance staff. In this situation, oxygen-driven nebulisers may be used but should be limited to 6 min for patients with known COPD because the nebuliser mask delivers a high concentration of oxygen (about 60\%). Since most of the effective dose from a nebuliser chamber is delivered within $6 \mathrm{~min}$, limiting oxygendriven nebulisers to 6 min will minimise the risk of hypercapnic respiratory failure while delivering most of the nebulised drug dose. It must be recognised that patients with COPD may develop hypercapnia and respiratory acidosis if they are left on high-flow oxygen via a nebuliser mask system long after the nebulisation process has finished.

Evidence statement

- High-concentration oxygen therapy can cause hypercapnia within $15 \mathrm{~min}$ in acute COPD (evidence level 4).

- The use of oxygen to drive nebulisers is based on expert opinion and extrapolation from observational studies (evidence level 4).

Recommendations

R1: For patients with asthma, nebulisers should be driven by piped oxygen or from an oxygen cylinder fitted with a highflow regulator capable of delivering a flow rate of $>6 \mathrm{~L} / \mathrm{min}$. The patient should be changed back to his/her usual oxygen mask or cannulae when nebuliser therapy is complete. If the cylinder does not produce this flow rate, an air-driven nebuliser (with electrical compressor) should be used with supplemental oxygen by nasal cannulae at $2-6 \mathrm{~L} / \mathrm{min}$ to maintain an appropriate oxygen saturation level (grade D).

R2: When nebulised bronchodilators are given to patients with hypercapnic acidosis, they should be given using an ultrasonic nebuliser or else a jet nebuliser driven by compressed air and, if necessary, supplementary oxygen should be given concurrently by nasal cannulae to maintain an oxygen saturation of 88-92\%. The same precautions should be applied to patients who are at risk of hypercapnic respiratory failure prior to the availability of blood gas results and the oxygen saturation should be monitored continuously during treatment. Once the nebulised treatment is completed for patients at risk of hypercapnic respiratory failure, their previous targeted oxygen therapy should be reinstituted (grade D).

Good practice points

Do not allow hypoxaemia to occur while administering nebulised treatments:

$\checkmark$ For hypoxaemic patients, oxygen therapy should continue during nebulised treatments. 
Driving gas for nebulised treatment in ambulances:

$\checkmark$ During treatment by ambulance staff oxygen-driven nebulisers should be used for patients with asthma and may be used for patients with COPD in the absence of an air-driven compressor system. If oxygen is used for patients with known COPD, its use should be limited to $6 \mathrm{~min}$. This will deliver most of the nebulised drug dose but limit the risk of hypercapnic respiratory failure (section 10.4). Ambulance services are encouraged to explore the feasibility of introducing battery-powered, air-driven nebulisers or portable ultrasonic nebulisers.

\subsection{Delivery of helium-oxygen mixtures (Heliox)}

The use of helium-oxygen mixtures is discussed in section 8.18. The evidence base for the use of this gas mixture is weak and the use of Heliox as an inspired gas or as the driving gas for nebuliser therapy is not recommended outside of randomised clinical trials. The use of Heliox affects the delivery of aerosolised treatment in complex ways so the choice of nebuliser systems for the use of Heliox as a driving gas in clinical trials is outside the scope of this guideline. The most likely use for Heliox in clinical practice is for patients with mechanical upper airway obstruction or postoperative stridor. It has been shown that Heliox administration via a standard high-concentration reservoir mask leads to significant dilution by room air. ${ }^{489}$ For this reason, Heliox should be administered via a system that achieves a gas tight seal, with no leaks between the delivery device and the surroundings.

Heliox 80:20 mixture has a similar oxygen content to air (nitrogen:oxygen 79:21). Heliox 70:30 delivers a higher $\mathrm{FiO}_{2}$ but is more dense than Heliox 80:20 because it contains more oxygen which is denser than helium. The overall balance between oxygen content and gas density has not been addressed in clinical trials but it is recommended that clinicians should aim to keep the saturation in the appropriate target range for the individual patient, usually $94-98 \%$ for patients with asthma or upper airway obstruction and $88-92 \%$ for patients with severe COPD.

\subsection{Delivery of nitrous oxide/oxygen mixtures (eg, Entonox)}

The use of nitrous oxide/oxygen mixtures (Entonox, a 50:50 mix) is discussed in section 8.18. The gas is supplied in a range of cylinder sizes ranging from small portable cylinders for ambulances to much larger ones that can allow the gas to be piped to various outlets around a labour ward. The gas mixture is usually self-administered using a facemask with a demand valve that the patient holds to their face. The demand valve opens to allow gas to flow when it detects an inspiration. Like oxygen, Entonox is classified as a medical gas, and although it is recommended that it is prescribed, it is not a prescription-only medicine. The manufacturers recommend that it should only be prescribed and administered by suitably trained personnel within established clinical guidelines or protocols.

\subsection{Delivery of oxygen during procedures that may} partially occlude the airway (eg, bronchoscopy)

Reduction in oxygen saturation is common during endoscopic procedures that may partially occlude the airway such as bronchoscopy and upper GI endoscopy, and supplemental oxygen is recommended in some guidelines for all patients given sedation and selected patients without sedation. ${ }^{402} 490491$ This guideline emphasises the importance of monitoring oxygenation during the procedure by use of continuous pulse oximetry (see section 8.16).

All guidelines stress caution with the use of sedation and supplemental oxygen in those at risk of hypercapnia. The BSG guidelines recommend preoxygenation at $2 \mathrm{~L} / \mathrm{min}$ to prevent hypoxaemia, claiming that this is safe even in patients with COPD. ${ }^{491}$ However, the authors of this guideline recommend that oxygen should be administered only to correct hypoxaemia. Particular caution is advised in patients who may be prone to carbon dioxide retention and the use of transcutaneous $\mathrm{CO}_{2}$ monitoring in these patients has been suggested. ${ }^{492}$

Generally, oxygen is delivered at a flow rate of at least $2 \mathrm{~L} /$ min via nasal cannulae through one or both nostrils to maintain oxygen saturations above $90 \%$ or to correct falls in saturation of $>4 \%$ as discussed in section 8.16. Patient positioning (supine or semirecumbent) had no significant effect on oxygen saturations during bronchoscopy in a study of 38 patients. ${ }^{393}$

Evidence statement

- The use of oxygen during endoscopic procedures is based on expert opinion and extrapolation from observational studies (evidence level 4).

Recommendations J2-J6

J2: All procedures involving conscious sedation warrant routine continuous monitoring of oxygen saturation via pulse oximetry prior to and during the procedure, and in the recovery period, particularly fibre-optic bronchoscopy and upper GI endoscopy where a reduction in $\mathrm{SaO}_{2}$ is common, particularly with concurrent use of sedation.

J3: Significant arterial oxygen desaturation $\left(\mathrm{SpO}_{2}<90 \%\right.$ or fall of $4 \%$ or more that is prolonged ( $>1$ min during endoscopy procedures)) should be corrected by supplemental oxygen with the aim of achieving target oxygen saturations of $94-98 \%$, or 88-92\% in those at risk of hypercapnic respiratory failure.

J4: Complicated upper GI endoscopy or procedures in patients with cardiorespiratory comorbidity are especially likely to lead to hypoxaemia and may also lead to hypercapnia, especially if the patient is heavily sedated. It is recommended that blood gases should be measured if such patients should require prolonged oxygen administration. The routine administration of oxygen is not recommended as it may delay the recognition of respiratory failure.

J5: Constant clinical assessment of the patient is crucial at all stages of conscious sedation procedures and monitoring of capnography or transcutaneous carbon dioxide levels may be a useful adjunct to identify early respiratory depression.

J6: During the recovery period after procedures requiring conscious sedation, supplemental oxygen should be titrated to achieve target saturations of $\mathbf{9 4 - 9 8 \%}$ in most patients and 88$92 \%$ in those at risk of hypercapnic respiratory failure (see section 10.5.1).

\subsection{CPAP devices and indications}

CPAP is a form of non-invasive ventilatory support that may be used when high inspired oxygen concentrations $(>60 \%)$ are not sufficient to maintain adequate oxygenation in patients with respiratory failure without hypercapnia. CPAP maintains a fairly constant positive pressure throughout inspiration and expiration, and the positive pressure applied is thought to improve lung expansion, recruit atelectactic areas and offset intrinsic PEEP, reducing work of breathing. Conditions in which CPAP has been used successfully in the management of acute respiratory failure include heart failure, pneumonia, ARDS, chest wall trauma and pulmonary fibrosis avoiding intubation and mechanical ventilation. 
The indications, use and equipment required for CPAP are more fully described in the BTS guideline for NIV. ${ }^{209}$ Generally, a gas flow generator is required, usually powered by the wall air and oxygen supplies or oxygen cylinder, and delivered by a tightly fitting facial or nasal mask, or hood. The level of positive pressure can be adjusted by the use of different sized valves fitted to the mask, or by a single variable valve. Domiciliary CPAP machines used for the treatment of OSA are not suitable for the management of respiratory failure as they may not be able to maintain pressure in patients with rapid respiratory rates and high minute ventilation. Non-invasive ventilators that are designed to deliver BiPAP can be used to deliver CPAP by setting the same inspiratory and expiratory pressure.

\section{SECTION 11: PRESCRIPTION, ADMINISTRATION AND MONITORING OF OXYGEN THERAPY}

\subsection{Safe prescription and administration of oxygen}

11.1.1 Legal status of medical oxygen: Does it need a prescription?

Medical oxygen is a drug. However, the legal status of oxygen in the UK is that of a medicinal product on the General Sales List (Medicines and Healthcare Products Regulatory Agency, personal communication). This means that the sale or dispensation of oxygen does not technically require a prescription because it is not a 'prescription-only medicine'. This status was conferred for practical reasons to facilitate the use of oxygen in the domiciliary setting where the distribution system no longer involves pharmacies. However, the use of oxygen by paramedics, registered nurses, doctors, physiotherapists and others in emergency situations is similar to the use of all other medicinal products by these people. Clinical governance requires that the intentions of the clinician who initiates oxygen therapy should be communicated clearly to the person who actually administers oxygen to the patient and an accurate record must be kept of exactly what has been given to the patient. In this respect, oxygen is in the same category as paracetamol, aspirin, ibuprofen, antihistamines, antiemetics and many other medicines that do not require a prescription if purchased by a patient for his/ her own use but do require accurate documentation if administered by a health professional to a patient. In healthcare settings, all of these medicines are conventionally recorded on a 'prescription chart' or 'drug kardex' alongside drugs in the 'prescription-only' category such as antibiotics.

A hospital 'prescription chart' is a document in which doctors and other prescribing clinicians make a list of all drugs and medicinal products required by a patient and where registered nurses and other clinicians make a record of all drugs that have been administered to the patient. In many hospitals, the prescription and documentation of administration are recorded directly into the Electronic Patient Record. These documents are also used by hospital pharmacies to dispense medicines but, for technical reasons, these hospital documents (or the equivalent documents in primary care settings or the Patient Report Form (PRF) or electronic PRF used by the ambulance services) do not have the same legal status as a prescription that is given to a patient to take to a community pharmacist for the purposes of dispensing a medicine. The 'prescription' of oxygen in acute healthcare settings is therefore not technically a prescription for two reasons: (1) oxygen is not a prescription-only medicine; and (2) a hospital drug chart is not truly a prescription. The second issue applies to all medicines used in healthcare settings, not just to oxygen. However, the words 'prescription' or 'prescription chart' are widely used to describe the documents which record the use of medicines in UK hospitals and other healthcare settings. For this reason, the present guideline will use the term 'prescribe' and 'prescription chart' for all orders for oxygen use. The important issue is that there must be a clear written record of all medicinal products, including oxygen, which are given to a patient by healthcare professionals. Ideally, this document should be prepared at the time when oxygen therapy is started. In emergencies, however, clinicians will treat the patient first and subsequently make written records of all treatments given, including oxygen therapy.

\subsubsection{Reasons to prescribe oxygen therapy}

Oxygen is prescribed for hypoxaemic patients to increase $\mathrm{PO}_{2}$ and decrease the work of breathing necessary to maintain a given $\mathrm{PaO}_{2}$ (see section 6). The concentration of oxygen required depends on the condition being treated; an inappropriate concentration may have serious or even lethal effects. Appropriate target saturation ranges for common medical emergencies are given in section 8 of this guideline and in tables 1-4. Each hospital should have an agreed policy and protocol for oxygen prescribing to allow staff to adjust oxygen delivery devices and to give oxygen in emergency situations prior to the availability of a prescription. The use of a patient group direction (PGD) may be appropriate if other solutions have not worked in the clinical setting. Please see details at: https://www. nice.org.uk/guidance/MPG2 and http://www.medicinesresources. nhs.uk/en/Communities/NHS/PGDs/.

Although a PGD is not strictly required for medical gases (because they are not a prescription-only medicine) it is important for every organisation to have a written policy to regulate oxygen use up to time when a prescription is written, for example, for patients arriving by ambulance in the emergency department with oxygen use already initiated by the ambulance service. For some organisations, a PGD may be considered as the best way to address this important issue. BTS audits up to 2015 have shown that only $57 \%$ of patients using supplemental oxygen in UK hospitals had a valid prescription or written order. This is a situation that would not be tolerated for any other drug.

\subsubsection{Implementing an oxygen prescription policy}

Oxygen prescriptions may include initial mode of delivery and flow rate (eg, $4 \mathrm{~L} / \mathrm{min}$ via nasal cannulae). But the most important aspect of the prescription is to give a target range. The clinicians who administer oxygen (usually registered nurses, midwives or physiotherapists) should be trained and empowered to adjust the oxygen concentration upwards and downwards as necessary to maintain the patient in the target saturation range (including adjustments made during nebulised therapy driven by oxygen or nebulised therapy driven by air with simultaneous administration of oxygen by nasal cannulae). This will require all hospitals to have an agreed oxygen administration programme with universal access to educational materials about oxygen administration. The clinicians who monitor the oxygen saturation (often healthcare assistants) should be trained to inform those who have been trained to administer oxygen if the oxygen saturations fall above or below the target saturations. Those doing the monitoring should also understand the importance for the patient of keeping in the target range. Implementing this policy will require all organisations to have agreed procedures (which may include PGDs up to the time when a prescription is written) and training programmes for all clinical staff and regular training programmes in the safe use of oxygen and audit of outcomes.

Many hospitals have developed an 'admission bundle' for all patients admitted to hospital. This bundle may include checking 
for hospital-associated thrombosis risk, checking MRSA risk and the prescription of antithrombotic agents and anti-MRSA agents if appropriate. As part of this process, many hospitals now specify that an oxygen saturation target must be set for all patients on admission to hospital so that the nursing team will know the correct starting target range if oxygen therapy should suddenly become necessary (as well as making a callout to the medical team to assess the patient). Furthermore, this allows nurses making bedside observations to know if the oxygen saturation is appropriate for each patient whether or not the patient is receiving oxygen at the time when the observation is made. Some older people without respiratory problems may have oxygen saturation below $94 \%$ and the target range for such patients should be adjusted accordingly. It was reported in the 2011 BTS national emergency oxygen audit that almost half of UK hospitals now have a policy of setting a target saturation range for all patients at the time of admission to hospital. Policies of this sort will become even more important when the EWS system takes oxygen saturation (above or below target range) into account. ${ }^{16}$

\subsubsection{Administration and monitoring of oxygen therapy}

The appropriate device should be used to provide the prescribed oxygen and the effects should be monitored using pulse oximetry, monitoring of respiratory rate and close observation of the patient. Arterial or capillary blood gas analysis should be repeated if clinical progress is not satisfactory and in all cases of hypercapnia and acidosis.

\subsubsection{Education of health professionals}

The clinician or registered healthcare professional administering the oxygen therapy should be aware of the hazards of hypoxaemia and hyperoxaemia and the signs and symptoms of inadequate or excessive oxygen delivery.

\subsubsection{How to prescribe oxygen effectively}

In the past, oxygen was often not prescribed at all or prescribed on a standard hospital drug chart as 'oxygen'. It was unusual for the prescription to include full details of what device to use, what flow rate(s) to administer and whether the prescription was for a fixed concentration of oxygen or to aim at a specific oxygen saturation target. ${ }^{4-10}$

It has been shown that a purpose-designed oxygen prescription sheet can improve oxygen prescribing in the short term, but experience has shown that free-standing oxygen prescription charts are often forgotten and unused. ${ }^{7}$ Recent audit studies by members of the guideline group (part of annual BTS hospital oxygen audits) have shown improved standards of prescribing with the use of a preprinted section for oxygen use in all hospital drug charts and with prompted electronic prescribing of oxygen for all hospital patients. This system was further enhanced by setting a desired saturation range for each patient. Suggested target saturations for common medical conditions are given in sections 8 and 9 of this guideline. It is important that healthcare professionals where oxygen is administered are familiar with the optimal saturation ranges for common conditions (see online appendix 3), and it is also important that those delivering the oxygen are familiar with the equipment in use and the best types of device to deliver low-concentration, mediumconcentration and high-concentration oxygen therapy. Figure 19 shows a working example of a preprinted oxygen section for a hospital prescription chart, and figures 1 and 2 (charts 1 and 2) give advice to prescribers and advice to those delivering oxygen onwards.
The safe use of oxygen includes careful consideration of the appropriate delivery device (mask, cannulae, etc) together with an appropriate source of oxygen and an appropriate oxygen flow rate.

An oxygen target range should be prescribed for all hospital patients. The method and rate of oxygen delivery should be altered by registered nurses or other registered healthcare professionals in order to achieve the prescribed target saturation as per the hospital policy. For most conditions, oxygen should be prescribed to achieve a target saturation of $94-98 \%$ (or $88-92 \%$ for those at risk of hypercapnic respiratory failure). The nurse should sign this prescription chart or the electronic equivalent on every drug round. This signature on the drug chart confirms that the patient is either receiving appropriate oxygen therapy (the delivery device and flow rate should be documented alongside the $\mathrm{SpO}_{2}$ on the bedside observations chart or electronic observations system) or else has $\mathrm{SpO}_{2}$ within the target range on air at the most recent observation round. It is not necessary to measure oximetry during drug rounds but action may be required if the patient was documented to be outside of their target range at the most recent observations round and if no action has yet taken place.

Evidence statement

- Prescription of oxygen in acute illness is based on expert opinion (evidence level 4).

Recommendations for safe prescribing and safe administration of oxygen

S1: Every healthcare facility should have a standard oxygen prescription document or, preferably, a designated oxygen section on all drug-prescribing cards or guided prescription of oxygen in electronic prescribing systems (grade D).

S2: A prescription for oxygen should always be provided, except in sudden illness when it must be started immediately and documented retrospectively (grade D).

S3: Doctors and other prescribers should prescribe oxygen using a target saturation range (sections 8,9 and 11) and sign the drug chart or electronic prescribing order (grade D).

S4: An oxygen target saturation range should be prescribed for all patients who are admitted to hospital. This will ensure that every patient will receive appropriate oxygen therapy if it should be required. It will also ensure that all clinicians are aware of the appropriate oxygen target range for every patient under their care (grade D).

Good practice points related to prescribing and administering oxygen therapy to patients

$\checkmark$ Oxygen should be prescribed on the drug chart or electronic prescribing system using a target saturation range.

$\checkmark$ Oxygen should be prescribed to a target saturation range rather than prescribing a fixed concentration of oxygen or $\mathrm{FiO}_{2}$ (see recommendations A1, A2, A4 and A5).

$\checkmark$ For hypoxaemic patients, oxygen therapy should continue during other treatments such as nebulised therapy. Clinicians should assess the clinical status of the patient prior to prescribing oxygen and the patient's condition should be reassessed frequently during oxygen use (see recommendations B1-B3).

$\checkmark$ In most emergency situations, oxygen is given to patients immediately without a formal prescription. The lack of a prescription should never preclude oxygen being given when needed in an emergency situation. However, a subsequent written record must be made of what oxygen therapy has been given to every patient in a similar manner to the recording of all other emergency treatment.

$\checkmark$ If a patient has an oxygen alert card, initial oxygen therapy should be based on the guidance on the card until the results of blood gases are available. 
Figure 19 Working example of oxygen section for hospital prescription charts. *Saturation is indicated in almost all cases except for terminal palliative care.

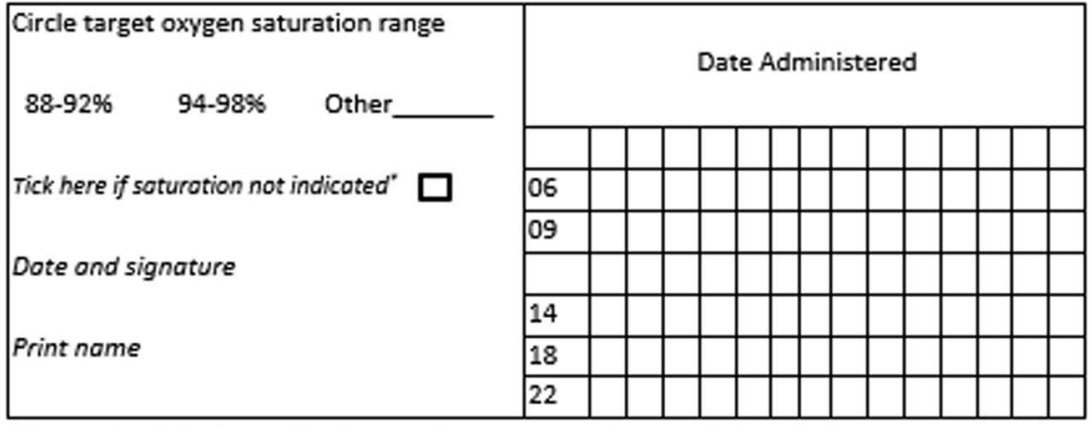

*Saturation is indicated in almost all cases except for terminal palliative care
Monitoring and adjusting therapy (see sections 9-11)

T1: Pulse oximetry must be available in all locations where emergency oxygen is being used by healthcare professionals (see also the limitations of using pulse oximetry section 7.1.2) (grade D).

T2: All documents which record oximetry measurements or blood gas results should state whether the patient is breathing air or a specified oxygen delivery device and flow rate using the abbreviations shown in table 5 (grade D).

T3: In all situations where repeated blood gas measurements are required, they should be measured as soon as possible, usually within $30 \mathrm{~min}$ of any treatment change, to determine if the proposed target saturations are appropriate. Consider the use of an indwelling arterial catheter if multiple samples are likely to be required (grade $\mathrm{D}$ ).

T4: Adjustments should only be made by registered staff who have been trained to administer oxygen. If the oxygen saturation falls below the prespecified range, the concentration of oxygen should be increased; if the saturation rises above this range, the oxygen concentration should be reduced. If the monitoring of oxygen saturation is performed by unregistered staff (eg, healthcare assistants), there must be a clear protocol in place which requires that they should inform staff who are trained to administer oxygen if the oxygen saturation is above or below the target saturation (grade D).

Good practice points related to administration of oxygen therapy $\checkmark$ For hypoxaemic patients, oxygen therapy should continue during other treatments such as nebulised therapy. Clinicians should assess the clinical status of the patient prior to prescribing oxygen and the patient's condition should be reassessed frequently during oxygen use (see recommendations B1-B3).

$\checkmark$ The administering healthcare professional should note the oxygen saturation before stating oxygen therapy whenever possible but never discontinue or delay oxygen therapy for seriously ill patients (see recommendation B2).

$\checkmark$ The healthcare professional should start oxygen therapy using an appropriate delivery system and flow rate as specified in sections 8-10 of this guideline. The target oxygen saturation should be documented on the respiratory section of the observation chart.

$\checkmark$ Whenever possible, patients should be given an oxygen information sheet (example in web appendix 6 of this guideline on the BTS website).

$\checkmark$ Staff should check the oxygen supply and connections on a regular basis because there have been serious incidents due to disconnection or misconnection of oxygen supplies.

$\checkmark$ Staff must ensure that adequate oxygen is provided during transfers and while patients are in diagnostic departments. Additionally, oxygen saturation should be monitored continuously for seriously ill patients who require escorted transfers. This is because there have been serious incidents involving accidental discontinuation of oxygen or cylinders running out during interward transfers or transfers to other departments such as for $\mathrm{x}$-rays.

Practical aspects of oxygen dispensing, documentation and monitoring

W1: Registered nurses and others who dispense drugs in hospitals should sign the drug chart or electronic prescribing record at every drug round and check that the patient is receiving oxygen therapy. This is to check that the patient is within the target saturation and also to check whether weaning and discontinuation should be instituted (grade D).

W2: Most patients are prescribed an oxygen target range. If patients are on air at the time of the drug round, registered nurses should sign the drug chart using a code such as ' $A$ ' for air and the observation chart should also be filled in using the code A for air (see table 5 and figure 19; grade D).

W3: All patients should have their oxygen saturation observed for at least $5 \mathrm{~min}$ after starting oxygen therapy or for patients who require an increased concentration of oxygen and after oxygen therapy has been decreased or stopped (grade D).

W4: If the oxygen saturation is above the target saturation range and the patient is stable, the delivery system or oxygen flow rate should be modified to return the saturation to within the target range (grade D).

W5: Patients who have a target saturation of $88-92 \%$ should have their blood gases measured within $30-60 \mathrm{~min}$. This is to ensure that the carbon dioxide level is not rising. This recommendation also applies to those who are at risk of developing hypercapnic respiratory failure but who have a normal $\mathrm{PCO}_{2}$ on the initial blood gas measurement (grade D).

W6: Stable patients whose oxygen saturation is within their target saturation range of $94-98 \%$ do not need repeat blood gas measurements within 30-60 min if there is no risk of hypercapnic respiratory failure and acidosis and may not need any further blood gas measurements unless there should be further deterioration including symptoms or signs of possible hypercapnia (grade D).

W7: Stable patients on oxygen treatment should have $\mathrm{SpO}_{2}$ and physiological variables (eg, NEWS) measured four times a day (grade D).

W8: In those who have signs of critical illness (eg, NEWS 7 or above), oxygen saturation should be monitored continuously and the patient may require level 2 or 3 care on a HDU or critical care unit (grade D).

W9: If the patient is clinically stable and the oxygen saturation is within the target range, treatment should be continued (or eventually lowered) depending on the clinical situation (grade D). 
W10: Oxygen therapy should be increased if the saturation is below the desired range and decreased if the saturation is above the desired range (and eventually discontinued as the patient recovers) (grade $\mathrm{D})$.

W11: The new saturation (and the new delivery system) and flow rate should be recorded on the patient's observation chart after $5 \mathrm{~min}$ of treatment at the new oxygen concentration. Each change should be recorded by the clinician trained to administer oxygen by signing the observation chart (only changes should be signed for) (grade D).

W12: Repeat blood gas measurements are not required for stable patients who require a reduced concentration of oxygen (or cessation of oxygen therapy) to maintain the desired target saturation (grade D).

W13: Patients with no risk of hypercapnic respiratory failure do not always need repeat blood gas measurements after an increase in oxygen concentration. However, the patient requires clinical review to determine why the oxygen saturation has fallen (grade D).

W14: Patients at risk of hypercapnic respiratory failure (usually those with a target range of $88-92 \%$; see table 4) require repeat blood gas assessment 30-60 min after an increase in oxygen therapy (to ensure that the carbon dioxide level is not rising) (grade D).

W15: For patients with no risk of hypercapnic respiratory failure, monitoring by pulse oximeter is sufficient (repeated blood gases not required) provided the patient is clinically stable and the oxygen saturation remains in the desired range, usually 94-98\% (grade D).

W16: If a patient's oxygen saturation is lower than the prescribed target range, first check all aspects of the oxygen delivery system and the oximeter device for faults or errors (grade D).

W17: If a patient's oxygen saturation is consistently lower than the prescribed target range, there should be a medical review and the oxygen therapy should be increased according to an agreed written protocol (grade D).

W18: If the oxygen saturation fails to rise following 5$10 \mathrm{~min}$ of increased oxygen therapy or if there is clinical concern following medical review, then blood gas measurements should be repeated (grade D).

\section{Training in oxygen prescribing and use}

$X 1$ : All clinicians prescribing oxygen should have appropriate training and access to written or electronic oxygen prescribing guidelines based on this national guideline (grade $\mathrm{D}$ ).

(Training slides for doctors and nurses are available as online supplementary appendices 7 and 8 on the BTS website.)

$X 2$ : Every hospital should have a training programme to ensure that clinical staff are familiar with the hospital's oxygen administration policies. In view of the high number of adverse incidents related to oxygen therapy, it is recommended that all acute Trusts should include basic training in oxygen use in the mandatory training programmes for all clinical staff (grade D).

\subsection{Starting oxygen therapy}

Safe prescribing and safe administration of oxygen are closely linked. In emergencies, oxygen therapy should be started immediately and documented as soon as possible. In all other situations, oxygen should be prescribed in accordance with the standards described in section 11.1 before administration is started. The healthcare professional who administers the oxygen therapy (usually a nurse or physiotherapist) should be fully trained and should follow local or national protocols as described in section 11.1.
Good practice points

$\checkmark$ The administering healthcare professional should note the oxygen saturation before starting oxygen therapy (see recommendation B2).

$\checkmark$ The healthcare professional should start oxygen therapy using an appropriate delivery system and flow rate as specified in sections $8-10$ of this guideline. The target oxygen saturation should be documented on the respiratory section of the observation chart.

$\checkmark$ Whenever possible, patients should be given an oxygen information sheet (example in web appendix 6 of this guideline on the BTS website).

\subsection{Monitoring oxygen therapy}

11.3.1 Pulse oximeters

Pulse oximetry should be available to all healthcare staff managing patients receiving oxygen therapy and they should be trained in their use (see section 7 for technical and practical information regarding oximeter use). Clinicians should be aware that pulse oximetry gives no information about the $\mathrm{PCO}_{2}$ or $\mathrm{pH}$ and most pulse oximeters are unreliable when a patient's $\mathrm{SpO}_{2}$ falls below about $85 \%$. Pulse oximetry is dependent on pulsatile flow, and it may not be possible to achieve a satisfactory oximeter reading in patients with cold hands, especially those with severe Raynaud's phenomenon due to collagen vascular diseases (which may also cause hypoxic lung disease). The readings may also be affected by shock, skin pigmentation, nail varnish, etc (see section 7). It is essential to record the oxygen delivery system alongside the oximetry result.

All measurements of oxygen saturation should be recorded in the observation chart along with the code for the oxygen delivery system that is being used. If the patient is breathing air at the time of the observation, this should also be recorded in the chart (see recommendation B2 and figure 19).

\subsubsection{Arterial or arterialised capillary blood gases}

- Arterial or arterialised capillary blood gases should be measured and the oxygen device and flow rate should be noted on arrival at hospital (or at the time when oxygen therapy becomes necessary) for most patients requiring emergency oxygen therapy (see recommendations C1-C3).

- Blood gas measurements should be repeated in all critically ill patients and in many other cases according to the response to treatment (see recommendations $\mathrm{C} 1-\mathrm{C} 3$ ).

\subsubsection{Physiological monitoring: 'track and trigger' systems}

- EWS systems such as the NEWS are useful for monitoring patients.

- Tachypnoea is a sensitive indicator of deteriorating respiratory function.

- All acutely ill patients should have physiological monitoring using EWS or a similar physiological assessment system in addition to pulse oximetry (see recommendation B3).

- The original 2012 version of the NEWS allocated inappropriate points for hypoxaemia to patients with target ranges below 94-98\% who were within their target range. It was necessary to correct for this when using such charts. ${ }^{165}$ This is recognised in the 2017 update of the NEWS chart which makes special provision for patients at risk of hypercapnia. Patients at risk of hypercapnia should score no NEWS points for saturation if within their target range and they should score points if the oxygen saturation falls below the target range or if the saturation rises above the target range while breathing oxygen. 
11.3.4 Monitoring during the first hour of oxygen therapy Evidence statement

- Monitoring of oxygen therapy is based on expert opinion.

- All patients should have their oxygen saturation observed for at least $5 \mathrm{~min}$ after starting oxygen therapy or for patients who require an increased concentration of oxygen and after oxygen therapy has been decreased or stopped (see recommendation W3) (evidence level 4).

\subsubsection{Subsequent monitoring}

The exact requirements for monitoring will depend on the clinical condition of each patient. Saturations are usually measured after 1 hour of oxygen therapy and then four-hourly. Stable patients should be monitored four times a day. However, critically ill patients will require continuous monitoring of oxygen saturation and other physiological measurements.

\subsubsection{When to increase oxygen therapy}

In most instances, failure to achieve the desired oxygen saturation is due to the severity of the patient's illness, but it is important to check that the oximeter is correctly placed and functioning normally as well as checking that the oxygen delivery device and the oxygen flow rate are correct. If the oxygen is being delivered from a cylinder, clinicians should check the labelling of the cylinder to ensure that it is an oxygen cylinder and checks should be made to ensure that the cylinder is not empty or near empty.

\subsubsection{When to lower oxygen therapy}

Most conditions which require supplemental oxygen therapy will improve with treatment and it will then be possible to reduce the amount of oxygen administered to the patient. Improvement will usually be confirmed by observing an improving oxygen saturation and a reduction in the physiological score on the NEWS observation chart as discussed in section 7.

Evidence statement

- Adjustment of oxygen therapy is based on expert opinion (evidence level 4).

Recommendations

$U 1$ : Lower the oxygen concentration if the patient is clinically stable and the oxygen saturation is above the target range or if it has been in the upper zone of the target range for some time (usually 4-8 hours) (grade D).

$U 2$ : If the target saturation is maintained, the new delivery system and flow should be continued. Repeat blood gas measurements are not required. If the patient is stable, the process can be repeated and the patient can eventually be weaned off oxygen (see section 12) (grade D).

\section{SECTION 12: WEANING AND DISCONTINUATION OF OXYGEN THERAPY}

In most acute illnesses, oxygen therapy will be reduced gradually as the patient recovers and oxygen therapy can be discontinued once the patient can maintain a saturation of $94-98 \%$ while breathing air (or the patient's baseline oxygen saturation level if known). Some patients may have episodic hypoxaemia during recovery from acute illness (eg, patients with COPD with intermittent mucus plugging) and some convalescent patients may be comfortable at rest with a satisfactory oxygen saturation but may desaturate and become breathless when they start to mobilise. The ongoing prescription of a target saturation range will cover both of these types of occurrence.

Some patients with chronic lung diseases will already be established on long-term oxygen therapy and should be tapered slowly to their usual maintenance oxygen delivery device and flow rate.

A small number of patients who have suffered major respiratory or cardiac injury may require a prescription for home oxygen to permit safe discharge from hospital. However, many patients with COPD exacerbations may have a low $\mathrm{PO}_{2}$ on discharge from hospital but a reasonable $\mathrm{PO}_{2}$ at a subsequent clinic visit, so decisions about long-term oxygen should not be made on the basis of blood gas measurements made during AECOPD (see BTS guideline for home oxygen in adults). ${ }^{13}$

\subsection{How to discontinue oxygen therapy for stable patients} Evidence statement

- Reduce oxygen therapy gradually for stable patients (see section 11.3.7). Weaning and discontinuation of oxygen therapy is based on expert opinion in the absence of randomised trials (evidence level 4).

Recommendations

U3: Most stable convalescent patients will eventually be stepped down to $2 \mathrm{~L} / \mathrm{min}$ via nasal cannulae prior to cessation of oxygen therapy. Patients at risk of hypercapnic respiratory failure may be stepped down to $1 \mathrm{~L} / \mathrm{min}$ (or occasionally $0.5 \mathrm{~L} /$ min) via nasal cannulae or a $24 \%$ Venturi mask at $2 \mathrm{~L} / \mathrm{min}$ as the lowest oxygen concentration prior to cessation of oxygen therapy (grade D).

U4: Oxygen therapy should be stopped once a patient is clinically stable on low-concentration oxygen and the oxygen saturation is within the desired range on two consecutive observations (but the prescription for a target saturation range should remain active in case of future deterioration). It may be appropriate to alter the target range following senior review in patients with chronic cardiopulmonary disease who either have saturations $<94 \%$ when stable or in whom it is deemed sensible to discharge from hospital with saturations $<94 \%$ pending an outpatient oxygen assessment. Oxygen should also be stopped if the patient has come to the end of a written protocol of timed oxygen (eg, postoperatively) (grade D).

U5: Oxygen saturation on air should be monitored for $5 \mathrm{~min}$ after stopping oxygen therapy. If it remains in the desired range it should be rechecked at 1 hour (grade D).

U6: If the oxygen saturation and physiological 'track and trigger' score (eg, NEWS) is satisfactory at 1 hour, the patient has safely discontinued oxygen therapy. However, saturation and physiology should continue to be monitored on a regular basis according to the patient's underlying clinical condition (grade D).

U7: If the saturation falls below the patient's target range on stopping oxygen therapy, restart the lowest concentration that maintained the patient in the target range and monitor for $5 \mathrm{~min}$. If this restores the saturation into the target range, continue oxygen therapy at this level and attempt discontinuation of oxygen therapy again at a later date provided the patient remains clinically stable (grade $\mathrm{D})$.

U8: If a patient requires oxygen therapy to be restarted at a higher concentration than before to maintain the same target saturation range, the patient should have a clinical review to establish the cause for this deterioration (grade D).

U9: Some patients may have episodic hypoxaemia (eg, after minor exertion or due to mucus plugging) after they have safely discontinued oxygen therapy. An ongoing prescription for a target saturation range will allow these patients to receive oxygen as the need arises but transient asymptomatic desaturation does not require correction (grade D). 


\section{SECTION 13: OUTCOMES AND AUDIT}

\subsection{Audit}

Audits of oxygen prescription and use have been carried by the BTS in 2008 prior to the launch of the first edition of this guideline and most years since then. ${ }^{16}$ About $14 \%$ of UK hospital patients receive supplemental oxygen therapy on any given day but only one-third of these patients had any type of prescription or written order for oxygen in 2008, this had risen slowly to only $57 \%$ by the time of the 2015 audit. ${ }^{493} 494$ Audit of oxygen use within individual healthcare organisations is strongly encouraged and the oxygen champion at each Trust can compare their results with the average for all other units.

\subsection{Audit of compliance with guideline}

It is recommended that all users of oxygen will audit their own practice against the suggested optimal practice suggested in this guideline. This applies especially to high-frequency users such as ambulance services, emergency departments and medical wards.

On the job audit

Regular reviews of the drug card and observation chart on medical rounds at the pilot sites have been very important in the successful introduction of this policy. This has provided instant feedback for doctors, nurses and healthcare assistants and has produced successful change.

\section{Whole hospital and ward audits}

An audit tool is available on the BTS audit website (https:// audits.brit-thoracic.org.uk/). This has been successfully used UK wide and since 2008 with annual nationwide audits and the facility for individual units to use the audit tool for a local audit at any time. The audit tool allows healthcare organisations to monitor how many patients are receiving oxygen therapy, whether there is a valid prescription and the proportion of patients within the target range. Healthcare organisations can also audit the documentation of oxygen saturation on observation rounds and whether oxygen has been signed for on drug rounds. Organisational issues can also be audited such as the following:

- Does the Trust have an oxygen prescribing policy based on this guideline?

- Is there a standard prompted oxygen in the prescription document or electronic record?

- Is training implemented for nursing staff?

- Is training implemented for medical staff?

\section{SECTION 14: DISSEMINATION AND IMPLEMENTATION OF THE GUIDELINE AND EDUCATION OF HEALTHCARE PROFESSIONALS}

These matters are covered in more detail in online appendix 11.

\subsection{Dissemination}

Dissemination of this guideline will be encouraged and supported strongly by the societies involved in the production of the guideline.

\subsection{Local guidelines}

It is recognised that many healthcare organisations tend to modify national guidelines for local use. Summaries of the guideline for acute hospitals, ambulance services and general practice are shown in online supplementary appendices 3 and 5 on the BTS website (www.brit-thoracic.org.uk). Educational materials are available on the BTS website (appendices 7 and 8). It is hoped that the shortened version of this guideline (or a customised local version) will be made available on the website of every NHS Trust.

\subsection{Local oxygen policy}

It is usual for a new policy to be presented to the local policy committee. A specimen example of a local policy is available in web appendix 4 to help with the production of this policy in individual healthcare organisations.

\subsection{Oxygen prescription chart}

Implementation of the guideline requires an 'oxygen section' in the prescription chart or in the electronic prescribing record in all hospitals. A specimen example is available in figure 19. From experience, it is recommended that oxygen should be placed at the start of the prescription chart because all hospital patients need a specified target saturation range. The oxygen prescription may be missed if it is placed in another part of the drug chart.

\subsection{Staff education}

Medical staff education is fundamental to the success of this guideline. Teaching slides are available on the BTS website. It is thought that these are suitable for FY1, FY2 and specialty trainees. They are also suitable for undergraduate medical education. Nursing staff and nursing students also require education as do physiotherapists, pharmacists, midwives and other clinicians including healthcare assistants and other unregistered staff who may assist in the care of patients who are using supplemental oxygen. Slide sets have also have also been produced for this purpose and are available on the BTS website. It is suggested that small groups of 5-10 nurses from wards are taught in sessions lasting $30 \mathrm{~min}$ each day before the introduction of the guideline locally. This has been found to be more successful than relying on training days as this would take too long to train enough staff adequately.

\subsection{Local champions}

Local champions exist in hospitals, local champions in hospitals, PCT providers and ambulance services who have helped to introduce these guidelines. The Guideline Development Group are very grateful to the local oxygen champions for organising the introduction of local oxygen policies in $89 \%$ of UK acute hospital Trusts by 2011. In most instances, the oxygen champions have introduced a new prescription chart and helped to organise staff education. Clinical governance leads will also need to become committed to the implementation of the Emergency Oxygen Guideline and audit of this process.

\subsection{Benefits of nationwide implementation}

One major benefit of nationwide implementation is that, when staff transfer between organisations, they will be familiar with the oxygen prescription and administration system.

\section{SECTION 15: AREAS REQUIRING FURTHER RESEARCH}

Because of the life and death nature of many conditions for which emergency oxygen therapy is used, it seems that clinicians have been wary of conducting controlled trials of oxygen therapy for most of the commoner indications. Additionally, it can be difficult to obtain ethical approval for such studies because seriously ill patients are usually not able to provide informed consent.

Further research is required in many areas including:

Basic physiological studies 
- Effect of cigarette smoking on blood levels of oxygen and carbon dioxide in acute illness.

Clinical trials

- Randomised trial of 'precautionary use' of oxygen in critical illness compared with a conservative policy of monitoring carefully and giving titrated oxygen therapy oxygen only if the saturation falls below the target range (and trials to determine the best target range to aim for).

- RCTs of nasal high-flow humidified oxygen compared with NIV or high-concentration oxygen in critical illness.

- The optimal oxygen target values and their feasibility during CPR and immediately after a restoration of spontaneous circulation in order to improve survival and neurological outcome.

- Prospective studies of the effect of oxygen in nonhypoxaemic patients with major trauma and head injury.

- Use of oxygen in myocardial infarction (at date of guideline publication, AVOID study is published in 2016 and DETO2X-AMI trial is ongoing). ${ }^{330-333}$

- Use of oxygen in unstable coronary syndromes or type 2 myocardial infarction.

- Use of oxygen in chest pain of presumed cardiac origin.

- Studies to determine if different types of oxygen mask can affect clinical outcomes.

- Use of oxygen in obstetric emergencies.

- Prospective studies to determine the optimal target saturation range for patients not at risk of hypercapnia, for example, target range $92-96 \%$ vs $94-98 \%$.

- Use of nasal cannulae to deliver low-dose oxygen therapy (eg, nasal cannulae at $0.5-1.0 \mathrm{~L} / \mathrm{min}$ compared with $24 \%$ and $28 \%$ face masks).

- Prospective studies to establish the ideal target saturation range in patients with exacerbated COPD; for example, should the target range be $88-92 \%$ or slightly lower or slightly higher for optimal outcome?

- RCTs of nasal high-flow (but low concentration) humidified oxygen compared with NIV in patients exacerbated COPD with hypercapnic respiratory failure and $\mathrm{pH} 7.30-7.35$ following initial optimisation of treatment.

- RCTs of titrated oxygen therapy in acute presentations of patients with conditions associated with chronic respiratory failure (eg, neuromuscular disease or obesity-associated hypoventilation).

- RCTs of titrated oxygen therapy in patients presenting acutely with non-hypercapnic respiratory failure (eg, heart failure, pneumonia).

- Randomised trials to determine if nitrous oxide is an effective analgesic agent and if the oxygen component affects clinical outcomes.

- Randomised trials to determine if clinical outcomes can be improved by the use of Heliox mixtures and, if so, which conditions are likely to benefit.

- Use of oxygen as required for relief or breathlessness in nonhypoxaemic patients with acute illness.

- Effects of humidified high-flow oxygen on patient comfort.

- Effects of increased flow rates from Venturi masks on patient comfort and oxygen saturation.

- Further comparisons of high-flow nasal oxygen with reservoir masks and high-concentration Venturi masks.

Implementation studies

- Benefits of alert cards and personalised oxygen masks for patients with prior hypercapnic respiratory failure.

- Further studies of clinical outcomes of patients exposed to hyperoxia.
- Relationship between oxygen levels and outcomes in a wide range of conditions.

- Studies to determine if implementation of this guideline improves patient outcomes.

- Studies to determine the best way to help clinicians to comply with guideline recommendations.

Systematic reviews

- Outcomes from different levels of oxygen supplementation in AECOPD.

- Updated review of oxygen therapy in myocardial infarction.

- Review of oxygen therapy in stroke.

Collaborators BTS Emergency Oxygen Guideline Group: BROD, LH, JE, VM, Dr Sabrina Bajwah, Professor Richard Beasley, Dr Katrina Curtis, Professor Anthony Davison, Dr Alistair Dorward, Dr Chris Dyer, Angela Evans, Lucy Falconer, Clare Fitzpatrick, Dr Simon Gibbs, Dr Kim Hinshaw, Dr Robin Howard, Dr Binita Kane, Dr Jeff Keep, Carol Kelly, Hasanin Khachi, Dr Muhammed Asad Iqbal Khan,

Dr Roop Kishen, Leigh Mansfield, Dr Bruce Martin, Dr Fionna Moore, Dr Duncan Powrie, Dr Louise Restrick, Professor Christine Roffe, Dr Mervyn Singer, Dr Jasmeet Soar, Dr lain Small, Lisa Ward, David Whitmore, Professor Wisia Wedzicha, Dr Meme Wijesinghe.

Contributors BROD was lead author responsible for the overall document and production of supporting appendices. LH was lead author for sections 3-6. JE was lead author for section 14. VM was lead author for sections 9-11. All authors were responsible for the final approval of the guideline. Members of the guideline group and their contributions to individual sections of the guideline are listed in Annex 1.

Competing interests LH has received research funding support from Bayer PLC. VM has received lecture and meeting attendance support from Chiesi, GSK and AstraZeneca.

Provenance and peer review Not commissioned; externally peer reviewed.

\section{REFERENCES}

1 COPD NICE Clinical Guideline 101, 2010.

2 O'Driscoll BR, Howard LS, Davison AG, et al. BTS guideline for emergency oxygen use in adult patients. Thorax 2008;63(Suppl 6):vi1-68.

3 Hale KE, Gavin C, O'Driscoll BR. Audit of oxygen use in emergency ambulances and in a hospital emergency department. Emerg Med J 2008;25:773-6.

4 Wilson AT, Channer KS. Hypoxaemia and supplemental oxygen therapy in the first 24 hours after myocardial infarction: the role of pulse oximetry. $J R$ Coll Physicians Lond 1997:31:657-61.

5 Cook DJ, Reeve BK, Griffith LE, et al. Multidisciplinary education for oxygen prescription. A continuous quality improvement study. Arch Intern Med 1996:156:1797-801.

6 Small D, Duha A, Wieskopf B, et al. Uses and misuses of oxygen in hospitalized patients. Am J Med 1992;92:591-5.

7 Dodd ME, Kellet F, Davis A, et al. Audit of oxygen prescribing before and after the introduction of a prescription chart. BMJ 2000;321:864-5.

8 Howell M. An audit of oxygen prescribing in acute general medical wards. Prof Nurse 2001;17:221-4.

9 Boyle M, Wong J. Prescribing oxygen therapy. An audit of oxygen prescribing practices on medical wards at North Shore Hospital, Auckland, New Zealand. N Z Med J 2006;119:U2080.

10 Kbar FA, Campbell IA. Oxygen therapy in hospitalized patients: the impact of local guidelines. J Eval Clin Pract 2006;12:31-6.

11 AGREE II Instrument. Secondary AGREE II Instrument. http://www.agreetrust.org/ agree-ii/

12 JRCALC. UK Ambulance Services Clinical Practice Guidelines 2013. London: JRCALC, 2013

13 Hardinge M, Annandale J, Bourne $S$, et al. British Thoracic Society guidelines for home oxygen use in adults. Thorax 2015;70(Suppl 1):i1-43.

14 BTS. British Thoracic Society Guideline Production Manual. London: BTS, 2014:31.

15 Network SSIG. SIGN 50 methodology. Secondary SIGN 50 methodology 2011. http://sign.ac.uk/methodology/index.html

16 O'Driscoll BR, Howard LS, Bucknall C, et al. British Thoracic Society emergency oxygen audits. Thorax 2011:66:734-5.

17 Crapo RO, Jensen RL, Hegewald M, et al. Arterial blood gas reference values for sea level and an altitude of 1,400 meters. Am J Respir Crit Care Med 1999;160 (Pt 1):1525-31.

18 Witting MD, Scharf SM. Diagnostic room-air pulse oximetry: effects of smoking, race, and sex. Am J Emerg Med 2008;26:131-6.

19 Smith GB, Prytherch DR, Watson D, et al. S(p)O(2) values in acute medical admissions breathing air-implications for the British Thoracic Society guideline for emergency oxygen use in adult patients? Resuscitation 2012;83:1201-5. 
20 Hardie JA, Mørkve O, Ellingsen I. Effect of body position on arterial oxygen tension in the elderly. Respiration 2002;69:123-8.

21 Hardie JA, Vollmer WM, Buist AS, et al. Reference values for arterial blood gases in the elderly. Chest 2004;125:2053-60.

22 Guénard H, Marthan R. Pulmonary gas exchange in elderly subjects. Eur Respir J 1996:9:2573-7.

23 Blom H, Mulder M, Verweij W. Arterial oxygen tension and saturation in hospital patients: effect of age and activity. BMJ 1988;297:720-1.

24 Cerveri I, Zoia MC, Fanfulla F, et al. Reference values of arterial oxygen tension in the middle-aged and elderly. Am J Respir Crit Care Med 1995;152:934-41.

25 Hansen JE, Casaburi R. Patterns of dissimilarities among instrument models in measuring $\mathrm{PO2}, \mathrm{PCO} 2$, and $\mathrm{pH}$ in blood gas laboratories. Chest 1998;113:780-7.

26 Beall CM. Oxygen saturation increases during childhood and decreases during adulthood among high altitude native Tibetians residing at 3,800-4,200m. High Alt Med Biol 2000;1:25-32.

27 Beall CM. Andean, Tibetan, and Ethiopian patterns of adaptation to high-altitude hypoxia. Integr Comp Biol 2006;46:18-24.

28 Grocott MP, Martin DS, Levett DZ, et al. Arterial blood gases and oxygen content in climbers on Mount Everest. N Eng/ J Med 2009;360:140-9.

29 Bowton DL, Scuderi PE, Haponik EF. The incidence and effect on outcome of hypoxemia in hospitalized medical patients. Am J Med 1994;97:38-46.

30 Brain Trauma F, American Association of Neurological S, Congress of Neurological $S$, et al. Guidelines for the management of severe traumatic brain injury. IX. Cerebral perfusion thresholds. J Neurotrauma 2007;24(Suppl 1):S59-64.

31 Jubran A, Tobin MJ. Reliability of pulse oximetry in titrating supplemental oxygen therapy in ventilator-dependent patients. Chest 1990;97:1420-5.

32 Slutsky AS. Consensus conference on mechanical ventilation-January 28-30, 1993 at Northbrook, Illinois, USA. Part I. European Society of Intensive Care Medicine, the ACCP and the SCCM. Intensive Care Med 1994;20:64-79.

33 Gries RE, Brooks LJ. Normal oxyhemoglobin saturation during sleep. How low does it go? Chest 1996;110:1489-92.

34 Hurtado A, Aste-Salazar H. Arterial blood gases and acid-base balance at sea level and at high altitudes. J Appl Physiol 1948;1:304-25.

35 Stock MC, Downs JB, McDonald JS, et al. The carbon dioxide rate of rise in awake apneic humans. J Clin Anesth 1988;1:96-103.

36 Levin $\mathrm{KP}$, Hanusa $\mathrm{BH}$, Rotondi $\mathrm{A}$, et al. Arterial blood gas and pulse oximetry in initial management of patients with community-acquired pneumonia. J Gen Intern Med 2001;16:590-8.

37 Manley G, Knudson MM, Morabito D, et al. Hypotension, hypoxia, and head injury: frequency, duration, and consequences. Arch Surg 2001;136:1118-23.

38 Moed BR, Boyd DW, Andring RE. Clinically inapparent hypoxemia after skeletal injury. The use of the pulse oximeter as a screening method. Clin Orthop Relat Res 1993;293:269-73

39 Considine J. The reliability of clinical indicators of oxygenation: a literature review. Contemp Nurse 2005;18:258-67.

40 Brealey D, Singer M. Mitochondrial dysfunction in sepsis. Curr Infect Dis Rep 2003:5:365-71.

41 O'Driscoll BR, Rudenski A, Turkington PM, et al. An audit of hypoxaemia, hyperoxaemia, hypercapnia and acidosis in blood gas specimens. Eur Respir $J$ 2012;39:219-21.

42 Decalmer S, O'Driscoll BR. Oxygen: friend or foe in peri-operative care? Anaesthesia 2013;68:8-12.

43 Plant PK, Owen JL, Elliott MW. One year period prevalence study of respiratory acidosis in acute exacerbations of COPD: implications for the provision of non-invasive ventilation and oxygen administration. Thorax 2000;55:550-4.

44 Denniston AKO, O'Brien C, Stableforth D. The use of oxygen in acute exacerbations of chronic obstructive pulmonary disease: a prospective audit of pre-hospital and hospital emergency management. Clin Med (Lond) 2002;2:449-51.

45 Joosten SA, Koh MS, Bu X, et al. The effects of oxygen therapy in patients presenting to an emergency department with exacerbation of chronic obstructive pulmonary disease. Med J Aust 2007;186:235-8.

46 Roberts CM, Stone RA, Buckingham RJ, et al. Acidosis, non-invasive ventilation and mortality in hospitalised COPD exacerbations. Thorax 2011;66:43-8.

47 Wijesinghe $M$, Williams $M$, Perrin $K$, et al. The effect of supplemental oxygen on hypercapnia in subjects with obesity-associated hypoventilation: a randomized, crossover, clinical study. Chest 2011;139:1018-24.

48 Aubier M, Murciano D, Milic-Emili J, et al. Effects of the administration of $\mathrm{O} 2$ on ventilation and blood gases in patients with chronic obstructive pulmonary disease during acute respiratory failure. Am Rev Respir Dis 1980;122:747-54.

49 Dick CR, Liu Z, Sassoon CS, et al. 02-induced change in ventilation and ventilatory drive in COPD. Am J Respir Crit Care Med 1997;155:609-14.

50 Dunn WF, Nelson SB, Hubmayr RD. Oxygen-induced hypercarbia in obstructive pulmonary disease. Am Rev Respir Dis 1991;144(Pt 1):526-30.

51 Austin MA, Wills KE, Blizzard L, et al. Effect of high flow oxygen on mortality in chronic obstructive pulmonary disease patients in prehospital setting: randomised controlled trial. BMJ 2010;341:c5462.
52 Cutaia M, Rounds S. Hypoxic pulmonary vasoconstriction. Physiologic significance, mechanism, and clinical relevance. Chest 1990;97:706-18.

53 Wetzel RC, Herold CJ, Zerhouni EA, et al. Hypoxic bronchodilation. J Appl Physiol 1992:73:1202-6.

54 Fidone SJ, Gonzalez C. Initiation and control of chemoreceptor activity in the carotid body. In: Cherniack NS, Widdicombe J, eds. Handbook of physiology. Bethesda, MA: American Physiology Society, 1986:247-312.

55 Lahiri S, Mulligan E, Nishino T, et al. Relative responses of aortic body and carotid body chemoreceptors to carboxyhemoglobinemia. J Appl Physiol Respir Environ Exerc Physiol 1981;50:580-6.

56 Matsuoka T, Saiki C, Mortola JP. Metabolic and ventilatory responses to anemic hypoxia in conscious rats. J Appl Physiol 1994;77:1067-72.

57 Johannes T, Mik EG, Nohé B, et al. Acute decrease in renal microvascular PO2 during acute normovolemic hemodilution. Am J Physiol Renal Physiol 2007;292: F796-803.

58 Rosenberger C, Mandriota S, Jürgensen JS, et al. Expression of hypoxia-inducible factor-1alpha and -2alpha in hypoxic and ischemic rat kidneys. J Am Soc Nephrol 2002;13:1721-32.

59 Beasley R, McNaughton A, Robinson G. New look at the oxyhaemoglobin dissociation curve. Lancet 2006;367:1124-6.

60 Tyuma I. The Bohr effect and the Haldane effect in human hemoglobin. Jpn J Physiol 1984;34:205-16.

61 Kahler RL, Goldblatt A, Braunwald E. The effects of acute hypoxia on the systemic venous and arterial systems and on myocardial contractile force. I Clin Invest 1962;41:1553-63.

62 Heistad DD, Abboud FM. Dickinson W. Richards lecture: circulatory adjustments to hypoxia. Circulation 1980;61:463-70.

63 Clifford PS, Hellsten Y. Vasodilatory mechanisms in contracting skeletal muscle. J Appl Physiol 2004;97:393-403.

64 Berne RM, Levy MN. Transport of oxygen and carbon dioxide: tissue oxygenation. In: Berne RM, Levy MN, ed. Physiology. Philadelphia: Mosby, 2005.

65 Mitchell RA, Loeschcke HH, Massion WH, et al. Respiratory responses mediated through superficial chemosensitive areas on the medulla. J Appl Physiol 1963;18:523-33.

66 Dorrington KL, Talbot NP. Human pulmonary vascular responses to hypoxia and hypercapnia. Pflugers Arch 2004;449:1-15.

67 Balanos GM, Talbot NP, Dorrington KL, et al. Human pulmonary vascular response to $4 \mathrm{~h}$ of hypercapnia and hypocapnia measured using Doppler echocardiography. J Appl Physiol 2003;94:1543-51.

68 Lumb A. Nunn's applied respiratory physiology. 6th edn. Oxford: Butterworth Heinemann, 2005

69 Marks B, Mitchell DG, Simelaro JP. Breath-holding in healthy and pulmonary-compromised populations: effects of hyperventilation and oxygen inspiration. J Magn Reson Imaging 1997;7:595-7.

70 Danias PG, Stuber M, Botnar RM, et al. Navigator assessment of breath-hold duration: impact of supplemental oxygen and hyperventilation. AJR Am J Roentgenol 1998;171:395-7.

71 McCarthy RM, Shea SM, Deshpande VS, et al. Coronary MR angiography: true FISP imaging improved by prolonging breath holds with preoxygenation in healthy volunteers. Radiology 2003:227:283-8.

72 Hebert PC, Wells G, Blajchman MA, et al. A multicenter, randomized, controlled clinical trial of transfusion requirements in critical care. Transfusion requirements in critical care investigators, Canadian Critical Care Trials Group. N Engl I Med 1999;340:409-17.

73 Bishop MH, Shoemaker WC, Appel PL, et al. Prospective, randomized trial of survivor values of cardiac index, oxygen delivery, and oxygen consumption as resuscitation endpoints in severe trauma. J Trauma 1995;38:780-7.

74 Velmahos GC, Demetriades D, Shoemaker WC, et al. Endpoints of resuscitation of critically injured patients: normal or supranormal? A prospective randomized trial. Ann Surg 2000;232:409-18.

75 Kern JW, Shoemaker WC. Meta-analysis of hemodynamic optimization in high-risk patients. Crit Care Med 2002;30:1686-92.

76 Boyd 0. Optimisation of oxygenation and tissue perfusion in surgical patients. Intensive Crit Care Nurs 2003;19:171-81.

77 Hayes MA, Yau EH, Timmins AC, et al. Response of critically ill patients to treatment aimed at achieving supranormal oxygen delivery and consumption. Relationship to outcome. Chest 1993;103:886-95.

78 Hayes MA, Timmins AC, Yau EH, et al. Elevation of systemic oxygen delivery in the treatment of critically ill patients. N Eng/ I Med 1994;330:1717-22.

79 Gattinoni L, Brazzi L, Pelosi P, et al. A trial of goal-oriented hemodynamic therapy in critically ill patients. SvO2 Collaborative Group. N Engl J Med 1995:333:1025-32.

80 Hoffman CE, Clark RT Jr, Brown EB Jr. Blood oxygen saturations and duration of consciousness in anoxia at high altitudes. Am J Physiol 1946;145:685-92.

81 Comroe JH Jr, Bahnson ER, Coates EO Jr. Mental changes occurring in chronically anoxemic patients during oxygen therapy. J Am Med Assoc 1950;143:1044-8.

82 Boycott AE, Haldane JS. The effects of low atmospheric pressures on respiration. J Physiol 1908;37:355-77. 
83 Yoneda I, Tomoda M, Tokumaru O, et al. Time of useful consciousness determination in aircrew members with reference to prior altitude chamber experience and age. Aviat Space Environ Med 2000;71:72-6.

84 Murphy R, Driscoll P, O'Driscoll R. Emergency oxygen therapy for the COPD patient. Emerg Med J 2001;18:333-9.

85 Warrell DA, Edwards RH, Godfrey $\mathrm{S}$, et al. Effect of controlled oxygen therapy on arterial blood gases in acute respiratory failure. BMJ 1970;1:452-5.

86 Peacock AJ, Jones PL. Gas exchange at extreme altitude: results from the British 40th Anniversary Everest Expedition. Eur Respir J 1997;10:1439-44.

87 Campbell EJ. The J. Burns Amberson Lecture. The management of acute respiratory failure in chronic bronchitis and emphysema. Am Rev Respir Dis 1967:96:626-39.

88 Kilburn KH, Dowell AR. Renal function in respiratory failure. Effects of hypoxia, hyperoxia, and hypercapnia. Arch Intern Med 1971;127:754-62.

89 Henrion J, Schapira M, Luwaert R, et al. Hypoxic hepatitis: clinical and hemodynamic study in 142 consecutive cases. Medicine (Baltimore) 2003:82:392-406.

90 Henrion J, Minette P, Colin L, et al. Hypoxic hepatitis caused by acute exacerbation of chronic respiratory failure: a case-controlled, hemodynamic study of 17 consecutive cases. Hepatology 1999;29:427-33.

91 Akerø A, Christensen CC, Edvardsen A, et al. Hypoxaemia in chronic obstructive pulmonary disease patients during a commercial flight. Eur Respir $J$ 2005;25:725-30.

92 Humphreys S, Deyermond R, Bali I, et al. The effect of high altitude commercial air travel on oxygen saturation. Anaesthesia 2005;60:458-60.

93 Cottrell JJ, Lebovitz BL, Fennell RG, et al. Inflight arterial saturation: continuous monitoring by pulse oximetry. Aviat Space Environ Med 1995;66:126-30.

94 Quarterman CP, Thomas AN, McKenna M, et al. Use of a patient information system to audit the introduction of modified early warning scoring. J Eval Clin Pract 2005;11:133-8.

95 Subbe $C P$, Kruger $M$, Rutherford $P$, et al. Validation of a modified Early Warning Score in medical admissions. QJM 2001;94:521-6.

96 London RCOP. Standardising the assessment of acute-illness severity in the NHS NEWS score. London: RCP London, 2012.

97 Tan A, Schulze A, O'Donnell CPF, et al. Air versus oxygen for resuscitation of infants at birth. Cochrane Database Syst Rev 2005;(2):CD002273.

98 Kuisma M, Boyd J, Voipio V, et al. Comparison of 30 and the $100 \%$ inspired oxygen concentrations during early post-resuscitation period: a randomised controlled pilot study. Resuscitation 2006;69:199-206.

99 Grubb NR, Simpson C, Sherwood RA, et al. Prediction of cognitive dysfunction after resuscitation from out-of-hospital cardiac arrest using serum neuron-specific enolase and protein S-100. Heart 2007;93:1268-73.

100 Kranke P, Bennett M, Roeckl-Wiedmann I, et al. Hyperbaric oxygen therapy for chronic wounds. Cochrane Database Syst Rev 2004;(2):CD004123.

101 Whitney JD, Heiner S, Mygrant BI, et al. Tissue and wound healing effects of short duration postoperative oxygen therapy. Biol Res Nurs 2001;2:206-15.

102 Downs JB. Has oxygen administration delayed appropriate respiratory care? Fallacies regarding oxygen therapy. Respiratory care 2003;48:611-20.

103 Lopez-Majano V, Dutton RE. Regulation of respiration during oxygen breathing in chronic obstructive lung disease. Am Rev Respir Dis 1973;108:232-40.

104 Massaro DJ, Katz S, Luchsinger PC. Effect of various modes of oxygen administration on the arterial gas values in patients with respiratory acidosis. BMJ 1962;2:627-9.

105 Thomson AJ, Webb DJ, Maxwell SR, et al. Oxygen therapy in acute medical care. BMJ 2002;324:1406-7.

106 Beasley R, Aldington S, Robinson G. Is it time to change the approach to oxygen therapy in the breathless patient? Thorax 2007;62:840-1.

107 Niesters M, Mahajan RP, Aarts L, et al. High-inspired oxygen concentration further impairs opioid-induced respiratory depression. Br J Anaesth 2013;110:837-41.

108 Pain MC, Read DJ, Read J. Changes of arterial carbon-dioxide tension in patients with chronic lung disease breathing oxygen. Australas Ann Med 1965; 14:195-204.

109 Berry RB, Mahutte CK, Kirsch JL, et al. Does the hypoxic ventilatory response predict the oxygen-induced falls in ventilation in COPD? Chest 1993;103:820-4.

110 Erbland ML, Ebert RV, Snow SL. Interaction of hypoxia and hypercapnia on respiratory drive in patients with COPD. Chest 1990;97:1289-94.

111 Castaing Y, Manier G, Guénard $H$. Effect of $26 \%$ oxygen breathing on ventilation and perfusion distribution in patients with cold. Bull Eur Physiopathol Respir 1985;21:17-23.

112 Robinson TD, Freiberg DB, Regnis JA, et al. The role of hypoventilation and ventilation-perfusion redistribution in oxygen-induced hypercapnia during acute exacerbations of chronic obstructive pulmonary disease. Am J Respir Crit Care Med 2000;161:1524-9.

113 Feller-Kopman D, Schwartzstein R. The role of hypoventilation and ventilation-perfusion redistribution in oxygen-induced hypercapnia during acute exacerbations of chronic obstructive pulmonary disease. Am J Respir Crit Care Med 2001;163:1755.
114 Johnson JE, Peacock MD, Hayes JA, et al. Forced expiratory flow is reduced by $100 \%$ oxygen in patients with chronic obstructive pulmonary disease. South Med J 1995;88:443-9.

115 Jensen AG, Johnson A, Sandstedt S. Rebreathing during oxygen treatment with face mask. The effect of oxygen flow rates on ventilation. Acta Anaesthesio/ Scand 1991;35:289-92.

116 Kane B, Turkington PM, Howard LS, et al. Rebound hypoxaemia after administration of oxygen in an acute exacerbation of chronic obstructive pulmonary disease. BMJ 2011;342:d1557.

117 Rudolf $M$, Turner JA, Harrison BD, et al. Changes in arterial blood gases during and after a period of oxygen breathing in patients with chronic hypercapnic respiratory failure and in patients with asthma. Clin Sci 1979;57:389-96.

118 Bryan CL, Jenkinson SG. Oxygen toxicity. Clin Chest Med 1988:9:141-52.

119 Kallet RH, Matthay MA. Hyperoxic acute lung injury. Respir Care 2013;58: 123-41.

120 Saugstad OD. Bronchopulmonary dysplasia-oxidative stress and antioxidants Semin Neonatol 2003;8:39-49.

121 Cannon RO III. Mechanisms, management and future directions for reperfusion injury after acute myocardial infarction. Nat Clin Pract Cardiovasc Med 2005;2:88-94

122 Smith JL. The pathological effects due to increase of oxygen tension in the air breathed. J Physiol (Lond) 1899;24:19-35.

123 Griffith DE, Holden WE, Morris JF, et al. Effects of common therapeutic concentrations of oxygen on lung clearance of 99mTc DTPA and bronchoalveolar lavage albumin concentration. Am Rev Respir Dis 1986;134:233-7.

124 Fu ES, Downs JB, Schweiger JW, et al. Supplemental oxygen impairs detection of hypoventilation by pulse oximetry. Chest 2004;126:1552-8.

125 Doelman CJ, Bast A. Oxygen radicals in lung pathology. Free Radic Biol Med 1990;9:381-400.

126 Smith LL. Mechanism of paraquat toxicity in lung and its relevance to treatment. Hum Toxicol 1987;6:31-6.

127 Cersosimo RJ, Matthews SJ, Hong WK. Bleomycin pneumonitis potentiated by oxygen administration. Drug Intell Clin Pharm 1985;19:921-3.

128 Nader-Djalal N, Knight PR III, Thusu K, et al. Reactive oxygen species contribute to oxygen-related lung injury after acid aspiration. Anesth Analg 1998;87:127-33.

129 Knight PR, Kurek C, Davidson BA, et al. Acid aspiration increases sensitivity to increased ambient oxygen concentrations. Am J Physiol Lung Cell Mol Physiol 2000;278:L1240-7.

130 Refsum HE. Relationship between state of consciousness and arterial hypoxaemia and hypercapnia in patients with pulmonary insufficiency, breathing air. Clin Sci 1963;25:361-7.

131 Seevers $\mathrm{MH}$. The narcotic properties of carbon dioxide. NY State J Med 1944;44:597-602.

132 Dripps RD, Comroe JH Jr. The respiratory and circulatory response of normal man to inhalation of 7.6 and 10.4 per cent $\mathrm{CO} 2$ with a comparison of the maximal ventilation produced by severe muscular exercise, inhalation of $\mathrm{CO} 2$ and maximal voluntary hyperventilation. Am J Physiol 1947;149:43-51.

133 Sieker HO, Hickam JB. Carbon dioxide intoxication: the clinical syndrome, its etiology and management with particular reference to the use of mechanical respirators. Medicine (Baltimore) 1956;35:389-423.

134 Westlake EK, Simpson T, Kaye M. Carbon dioxide narcosis in emphysema. Q J Med 1955;24:155-73.

135 Cameron L, Pilcher J, Weatherall $M$, et al. The risk of serious adverse outcomes associated with hypoxaemia and hyperoxaemia in acute exacerbations of COPD. Postgrad Med J 2012;88:684-9.

136 Perrin K, Wijesinghe M, Healy B, et al. Randomised controlled trial of high concentration versus titrated oxygen therapy in severe exacerbations of asthma. Thorax 2011;66:937-41.

137 Wijesinghe M, Perrin K, Healy B, et al. Randomized controlled trial of high concentration oxygen in suspected community-acquired pneumonia. J $R$ Soc Med 2012;105:208-16.

138 Burri $\mathrm{E}$, Potocki $\mathrm{M}$, Drexler $\mathrm{B}$, et al. Value of arterial blood gas analysis in patients with acute dyspnea: an observational study. Crit Care 2011;15:R145.

139 Barer GR, Howard P, McCurrie JR. The effect of carbon dioxide and changes in blood pH on pulmonary vascular resistance in cats. Clin Sci 1967;32: $361-76$.

140 Kiely DG, Cargill RI, Lipworth BJ. Effects of hypercapnia on hemodynamic, inotropic, lusitropic, and electrophysiologic indices in humans. Chest 1996;109:1215-21.

141 Loeppky JA, Scotto P, Riedel CE, et al. Effects of acid-base status on acute hypoxic pulmonary vasoconstriction and gas exchange. J Appl Physiol 1992;72:1787-97.

142 Domino KB, Swenson ER, Hlastala MP. Hypocapnia-induced ventilation/perfusion mismatch: a direct $\mathrm{CO} 2$ or pH-mediated effect? Am J Respir Crit Care Med 1995; 152(Pt 1):1534-9.

143 Cullen DJ, Eger El II. Cardiovascular effects of carbon dioxide in man. Anesthesiology 1974;41:345-9.

144 Goldstein B, Shannon DC, Todres ID. Supercarbia in children: clinical course and outcome. Crit Care Med 1990;18:166-8. 
145 Norman JN, Macintyre J, Shearer JR, et al. Effect of carbon dioxide on renal blood flow. Am J Physiol 1970;219:672-6.

146 O'Neill B, Mahon JM, Bradley J. Short-burst oxygen therapy in chronic obstructive pulmonary disease. Respir Med 2006;100:1129-38.

147 Philip J, Gold M, Milner A, et al. A randomized, double-blind, crossover trial of the effect of oxygen on dyspnea in patients with advanced cancer. J Pain Symptom Manage 2006;32:541-50.

148 Clemens KE, Klaschik E. [Symptomatic treatment of dyspnoea in patients receiving palliative care: nasal delivery of oxygen compared with opioid administration]. Dtsch Med Wochenschr 2007;132:1939-43.

149 Clemens KE, Klaschik E. Symptomatic therapy of dyspnea with strong opioids and its effect on ventilation in palliative care patients. J Pain Symptom Manage 2007;33:473-81.

150 Abernethy AP, McDonald CF, Frith PA, et al. Effect of palliative oxygen versus room air in relief of breathlessness in patients with refractory dyspnoea: a double-blind, randomised controlled trial. Lancet 2010;376:784-93.

151 Kilgannon JH, Jones $\mathrm{AE}$, Shapiro $\mathrm{Nl}$, et al. Association between arterial hyperoxia following resuscitation from cardiac arrest and in-hospital mortality. JAMA 2010;303:2165-71.

152 Kilgannon JH, Jones AE, Parrillo JE, et al. Relationship between supranormal oxygen tension and outcome after resuscitation from cardiac arrest. Circulation 2011;123:2717-22.

153 de Jonge E, Peelen L, Keijzers PJ, et al. Association between administered oxygen, arterial partial oxygen pressure and mortality in mechanically ventilated intensive care unit patients. Crit Care 2008;12:R156.

154 Bellomo R, Bailey M, Eastwood GM, et al. Arterial hyperoxia and in-hospital mortality after resuscitation from cardiac arrest. Crit Care 2011:15:R90.

155 Eastwood G, Bellomo R, Bailey M, et al. Arterial oxygen tension and mortality in mechanically ventilated patients. Intensive Care Med 2012;38:91-8.

156 Janz DR, Hollenbeck RD, Pollock JS, et al. Hyperoxia is associated with increased mortality in patients treated with mild therapeutic hypothermia after sudden cardiac arrest. Crit Care Med 2012;40:3135-9.

157 Gattinoni L. Therapeutic approach to respiratory failure. In: Webb AS, Singer MJ, Suter PM, eds. Oxford texbook of critical care. Oxford: Oxford University Press, 1999:74-8

158 Uronis H, McCrory DC, Samsa G, et al. Symptomatic oxygen for non-hypoxaemic chronic obstructive pulmonary disease. Cochrane Database Syst Rev 2011;(6): CD006429.

159 Gallagher R, Roberts D. A systematic review of oxygen and airflow effect on relief of dyspnea at rest in patients with advanced disease of any cause. J Pain Palliat Care Pharmacother 2004;18:3-15.

160 Rowat AM, Wardlaw JM, Dennis MS, et al. Patient positioning influences oxygen saturation in the acute phase of stroke. Cerebrovasc Dis 2001;12:66-72.

161 Tyson SF, Nightingale P. The effects of position on oxygen saturation in acute stroke: a systematic review. Clin Rehabil 2004;18:863-71.

162 Bourke SJ, Munro NC, White JE, et al. Platypnoea-orthodeoxia in cryptogenic fibrosing alveolitis. Respir Med 1995:89:387-9.

163 Excellence NIfHaC. Acutely ill patients in hospital. Secondary Acutely ill patients in hospital 2007. http://publications.nice.org.uk/acutely-ill-patients-in-hospital-cc50

164 O'Driscoll R. Emergency oxygen use. BMJ 2012;345:e6856.

165 O'Driscoll BR, Bakerly ND, Murphy P, et al. Concerns regarding the design of the bedside monitoring chart for use with the NEWS (National Early Warning System). Clin Med (Lond) 2013;13:319-20.

166 Bota GW, Rowe BH. Continuous monitoring of oxygen saturation in prehospital patients with severe illness: the problem of unrecognized hypoxemia. J Emerg Med 1995:13:305-11.

167 Brown LH, Manring EA, Kornegay HB, et al. Can prehospital personnel detect hypoxemia without the aid of pulse oximeters? Am J Emerg Med 1996;14:43-4.

168 Lambert MA, Crinnion J. The role of pulse oximetry in the accident and emergency department. Arch Emerg Med 1989;6:211-15.

169 O'Driscoll BR, Murphy P, Turkington PM. Acute monitoring of patients with chronic respiratory disease during hospital admission. Clin Med (Lond) 2012;12:79-81.

170 Goodacre S, Turner J, Nicholl J. Prediction of mortality among emergency medical admissions. Emerg Med J 2006;23:372-5.

171 Thrush DN, Downs JB, Hodges M, et al. Does significant arterial hypoxemia alter vital signs? J Clin Anesth 1997;9:355-7.

172 Howes DW, Field B, Leary T, et al. Justification of pulse oximeter costs for paramedic prehospital providers. Prehosp Emerg Care 2000;4:151-5.

173 Macnab AJ, Susak L, Gagnon FA, et al. The cost-benefit of pulse-oximeter use in the prehospital environment. Prehosp Disaster Med 1999;14:245-50.

174 Jensen LA, Onyskiw JE, Prasad NG. Meta-analysis of arterial oxygen saturation monitoring by pulse oximetry in adults. Heart Lung 1998;27:387-408.

175 Aughey K, Hess D, Eitel D, et al. An evaluation of pulse oximetry in prehospital care. Ann Emerg Med 1991;20:887-91.

176 Modica R, Rizzo A. Accuracy and response time of a portable pulse oximeter. The Pulsox-7 with a finger probe. Respiration 1991;58:155-7.
177 Severinghaus JW, Naifeh KH. Accuracy of response of six pulse oximeters to profound hypoxia. Anesthesiology 1987;67:551-8.

178 Severinghaus JW, Naifeh KH, Koh SO. Errors in 14 pulse oximeters during profound hypoxia. J Clin Monit 1989:5:72-81.

179 Kelly AM, McAlpine R, Kyle E. How accurate are pulse oximeters in patients with acute exacerbations of chronic obstructive airways disease? Respir Med 2001;95:336-40.

180 Perkins GD, McAuley DF, Giles S, et al. Do changes in pulse oximeter oxygen saturation predict equivalent changes in arterial oxygen saturation? Crit Care 2003; 7:R67.

181 Pedersen T, Møller AM, Hovhannisyan K. Pulse oximetry for perioperative monitoring. Cochrane Database Syst Rev 2009;(4):CD002013.

182 Falconer RJ, Robinson BJ. Comparison of pulse oximeters: accuracy at low arterial pressure in volunteers. Br J Anaesth 1990;65:552-7.

183 Lee WW, Mayberry K, Crapo R, et al. The accuracy of pulse oximetry in the emergency department. Am J Emerg Med 2000;18:427-31.

184 Bickler PE, Feiner JR, Severinghaus JW. Effects of skin pigmentation on pulse oximeter accuracy at low saturation. Anesthesiology 2005;102:715-9.

185 Lee $\mathrm{KH}$, Hui KP, Tan WC, et al. Factors influencing pulse oximetry as compared to functional arterial saturation in multi-ethnic Singapore. Singapore Med $J$ 1993:34:385-7.

186 Zeballos RJ, Weisman IM. Reliability of noninvasive oximetry in black subjects during exercise and hypoxia. Am Rev Respir Dis 1991;144:1240-4.

187 Comber JT, Lopez BL. Evaluation of pulse oximetry in sickle cell anemia patients presenting to the emergency department in acute vasoocclusive crisis. Am J Emerg Med 1996;14:16-18.

188 Ortiz FO, Aldrich TK, Nagel RL, et al. Accuracy of pulse oximetry in sickle cell disease. Am J Respir Crit Care Med 1999;159:447-51.

189 Ahmed S, Siddiqui AK, Sison CP, et al. Hemoglobin oxygen saturation discrepancy using various methods in patients with sickle cell vaso-occlusive painful crisis. Eur J Haematol 2005;74:309-14.

190 Blaisdell CJ, Goodman S, Clark K, et al. Pulse oximetry is a poor predictor of hypoxemia in stable children with sickle cell disease. Arch Pediatr Adolesc Med 2000;154:900-3.

191 Barker SJ. "Motion-resistant" pulse oximetry: a comparison of new and old models. Anesth Analg 2002;95:967-72.

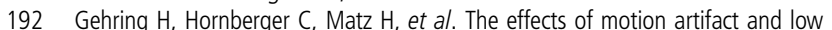
perfusion on the performance of a new generation of pulse oximeters in volunteers undergoing hypoxemia. Respir Care 2002;47:48-60.

193 Barker SJ, Hyatt J, Shah NK, et al. The effect of sensor malpositioning on pulse oximeter accuracy during hypoxemia. Anesthesiology 1993;79:248-54.

194 Hamber EA, Bailey PL, James SW, et al. Delays in the detection of hypoxemia due to site of pulse oximetry probe placement. I Clin Anesth 1999;11:113-18.

195 Tittle M, Flynn MB. Correlation of pulse oximetry and co-oximetry. Dimens Crit Care Nurs 1997;16:88-95.

196 Eaton T, Rudkin S, Garrett JE. The clinical utility of arterialized earlobe capillary blood in the assessment of patients for long-term oxygen therapy. Respir Med 2001;95:655-60.

197 Murphy R, Thethy S, Raby S, et al. Capillary blood gases in acute exacerbations of COPD. Respir Med 2006;100:682-6.

198 Zavorsky GS, Cao J, Mayo NE, et al. Arterial versus capillary blood gases: a meta-analysis. Respir Physiol Neurobiol 2007;155:268-79.

199 Wimpress S, Vara DD, Brightling CE. Improving the sampling technique of arterialized capillary samples to obtain more accurate $\mathrm{PaO} 2$ measurements. Chron Respir Dis 2005;2:47-50.

200 Pitkin $A D$, Roberts $C M$, Wedzicha JA. Arterialised earlobe blood gas analysis: an underused technique. Thorax 1994;49:364-6.

201 Dar K, Williams T, Aitken R, et al. Arterial versus capillary sampling for analysing blood gas pressures. BMJ 1995;310:24-5.

202 Lightowler JV, Elliott MW. Local anaesthetic infiltration prior to arterial puncture for blood gas analysis: a survey of current practice and a randomised double blind placebo controlled trial. J R Coll Physicians Lond 1997;31:645-6.

203 Giner J, Casan P, Belda J, et al. Pain during arterial puncture. Chest 1996;110:1443-5.

204 Ibrahim I, Yau YW, Ong L, et al. Arterial puncture using insulin needle is less painful than with standard needle: a randomized crossover study. Acad Emerg Med 2015;22:315-20.

205 Valentine RJ, Modrall JG, Clagett GP. Hand ischemia after radial artery cannulation. J Am Coll Surg 2005;201:18-22.

206 Tatevossian RG, Wo CC, Velmahos GC, et al. Transcutaneous oxygen and CO2 as early warning of tissue hypoxia and hemodynamic shock in critically ill emergency patients. Crit Care Med 2000;28:2248-53.

207 Slinger P, Blundell PE, Metcalf IR. Management of massive grain aspiration. Anesthesiology 1997;87:993-5.

208 Cham GWM, Tan WP, Earnest A, et al. Clinical predictors of acute respiratory acidosis during exacerbation of asthma and chronic obstructive pulmonary disease. Eur J Emerg Med 2002;9:225-32. 
209 British Thoracic Society Standards of Care Committee. Non-invasive ventilation in acute respiratory failure. Thorax 2002;57:192-211.

210 Kelly AM, Kyle E, McAlpine R. Venous pCO(2) and pH can be used to screen for significant hypercarbia in emergency patients with acute respiratory disease. J Emerg Med 2002;22:15-19.

211 Malatesha G, Singh NK, Bharija A, et al. Comparison of arterial and venous pH, bicarbonate, PCO2 and PO2 in initial emergency department assessment. Emerg Med J 2007:24:569-71.

212 Rang LC, Murray HE, Wells GA, et al. Can peripheral venous blood gases replace arterial blood gases in emergency department patients? CJEM 2002;4:7-15.

213 McCanny P, Bennett K, Staunton P, et al. Venous vs arterial blood gases in the assessment of patients presenting with an exacerbation of chronic obstructive pulmonary disease. Am J Emerg Med 2012;30:896-900.

214 McKeever TM, Hearson G, Housley $G$, et al. Using venous blood gas analysis in the assessment of COPD exacerbations: a prospective cohort study. Thorax 2016;71:210-15.

215 Lim BL, Kelly AM. A meta-analysis on the utility of peripheral venous blood gas analyses in exacerbations of chronic obstructive pulmonary disease in the emergency department. Eur J Emerg Med 2010;17:246-8.

216 Levine RL, Wayne MA, Miller CC. End-tidal carbon dioxide and outcome of out-of-hospital cardiac arrest. N Engl J Med 1997;337:301-6.

217 Grmec S, Klemen P. Does the end-tidal carbon dioxide (EtCO2) concentration have prognostic value during out-of-hospital cardiac arrest? Eur J Emerg Med 2001;8:263-9.

218 Ahrens T, Schallom L, Bettorf K, et al. End-tidal carbon dioxide measurements as a prognostic indicator of outcome in cardiac arrest. Am J Crit Care 2001;10:391-8.

219 Isserles SA, Breen PH. Can changes in end-tidal PCO2 measure changes in cardiac output? Anesth Analg 1991;73:808-14.

220 Kocher S, Rohling R, Tschupp A. Performance of a digital PCO2/SPO2 ear sensor. J Clin Monit Comput 2004;18:75-9.

221 Parker SM, Gibson GJ. Evaluation of a transcutaneous carbon dioxide monitor ("TOSCA") in adult patients in routine respiratory practice. Respir Med 2007;101:261-4.

222 Janssens JP, Howarth-Frey C, Chevrolet JC, et al. Transcutaneous PCO2 to monito noninvasive mechanical ventilation in adults: assessment of a new transcutaneous PCO2 device. Chest 1998:113:768-73.

223 Cox M, Kemp R, Anwar S, et al. Non-invasive monitoring of $\mathrm{CO} 2$ levels in patients using NIV for AECOPD. Thorax 2006;61:363-4.

224 Maniscalco M, Zedda A, Faraone $\mathrm{S}$, et al. Evaluation of a transcutaneous carbon dioxide monitor in severe obesity. Intensive Care Med 2008;34:1340-4.

225 Wijesinghe $\mathrm{M}$, Perrin $\mathrm{K}$, Weatherall $\mathrm{M}$, et al. A randomised controlled trial of high flow versus titrated oxygen therapy in the management of patients with community acquired pneumonia. Respirology 2009;14(Suppl 1):A83.

226 Perrin $\mathrm{K}$, Wijesinghe $\mathrm{M}$, Weatherall $\mathrm{M}$, et al. Assessing $\mathrm{PaCO} 2$ in acute respiratory disease: accuracy of a transcutaneous carbon dioxide device. Intern Med J 2011;41:630-3.

227 Lawson-Smith P, Jansen EC, Hyldegaard O. Cyanide intoxication as part of smoke inhalation - a review on diagnosis and treatment from the emergency perspective. Scand I Trauma Resusc Emerg Med 2011;19:14.

228 Raffin TA. Indications for arterial blood gas analysis. Ann Intern Med 1986:105:390-8.

229 BTS/SIGN. British Guideline for the Management of Asthma. SIGN, 2016:1-144.

230 Waldau T, Larsen VH, Bonde J. Evaluation of five oxygen delivery devices in spontaneously breathing subjects by oxygraphy. Anaesthesia 1998;53:256-63.

231 Soar J, Deakin C, Lockey A, et al. Resuscitation Guidelines 2015 Adult Life Support. London: Resuscitation Council UK, 2015.

232 Elmer J, Scutella M, Pullalarevu R, et al. The association between hyperoxia and patient outcomes after cardiac arrest: analysis of a high-resolution database. Intensive Care Med 2015;41:49-57.

233 Wang $\mathrm{CH}$, Chang WT, Huang $\mathrm{CH}$, et al. The effect of hyperoxia on survival following adult cardiac arrest: a systematic review and meta-analysis of observational studies. Resuscitation 2014;85:1142-8.

234 Forst $\mathrm{H}$. Maximising oxygen delivery in critically ill patients: a rational therapeutic approach (in German). Anaesthetist 1997;46:46-52.

235 Gueugniaud PY, Bertin-Maghit M, Hirschauer C, et al. In the early stage of major burns, is there a correlation between survival, interleukin-6 levels and oxygen delivery and consumption? Burns 1997:23:426-31.

236 Holm C, Melcer B, Hörbrand F, et al. The relationship between oxygen delivery and oxygen consumption during fluid resuscitation of burn-related shock. J Burn Care Rehabil 2000;21:147-54.

237 Steltzer $\mathrm{H}$, Hiesmayr $\mathrm{M}$, Mayer $\mathrm{N}$, et al. The relationship between oxygen delivery and uptake in the critically ill: is there a critical or optimal therapeutic value? A meta-analysis. Anaesthesia 1994;49:229-36.

238 Dellinger RP, Carlet JM, Masur H, et al. Surviving Sepsis Campaign guidelines for management of severe sepsis and septic shock. Crit Care Med 2004;32:858-73.

239 Reinhart K, Bloos F, König F, et al. Reversible decrease of oxygen consumption by hyperoxia. Chest 1991;99:690-4.
240 Rossi P, Tauzin L, Weiss M, et al. Could hyperoxic ventilation impair oxygen delivery in septic patients? Clin Physiol Funct Imaging 2007;27:180-4.

241 Dyson A, Stidwill R, Taylor V, et al. The impact of inspired oxygen concentration on tissue oxygenation during progressive haemorrhage. Intensive Care Med 2009;35:1783-91.

242 Damiani E, Adrario E, Girardis $\mathrm{M}$, et al. Arterial hyperoxia and mortality in critically ill patients: a systematic review and meta-analysis. Crit Care 2014;18:711.

243 Helmerhorst HJ, Roos-Blom MJ, van Westerloo DJ, et al. Association between arterial hyperoxia and outcome in subsets of critical illness: a systematic review, meta-analysis, and meta-regression of cohort studies. Crit Care Med 2015;43:1508-19.

244 Girardis M, Busani S, Damiani E, et al. Effect of conservative vs conventional oxygen therapy on mortality among patients in an intensive care unit: the oxygen-ICU randomized clinical trial. JAMA 2016;316:1583-9.

245 Dellinger RP, Levy MM, Rhodes A, et al. Surviving sepsis campaign: international guidelines for management of severe sepsis and septic shock: 2012. Crit Care Med 2013;41:580-637.

246 Mildh LH, Piilonen A, Kirvelä OA. Supplemental oxygen is not required in trauma patients treated with IV opiates. Am J Emerg Med 2003;21:35-8.

247 Price GF, Goodwin NM. Hypoxaemia and leucopaenia after fractures. Injury 1980;12:115-18.

248 Wong MWN, Tsui HF, Yung SH, et al. Continuous pulse oximeter monitoring for inapparent hypoxemia after long bone fractures. J Trauma 2004;56:356-62.

249 Szpilman D, Bierens JJ, Handley AJ, et al. Drowning. N Engl J Med 2012;366:2102-10.

250 Soar J, Pumphrey R, Cant A, et al. Emergency treatment of anaphylactic reactions — guidelines for healthcare providers. Resuscitation 2008;77:157-69.

251 Gentleman D. Causes and effects of systemic complications among severely head injured patients transferred to a neurosurgical unit. Int Surg 1992;77: 297-302.

252 Pfenninger EG, Lindner KH. Arterial blood gases in patients with acute head injury at the accident site and upon hospital admission. Acta Anaesthesiol Scand 1991;35:148-52.

253 Robertson C. Desaturation episodes after severe head injury: influence on outcome. Acta Neurochir Suppl (Wien) 1993;59:98-101.

254 Adamides AA, Winter CD, Lewis PM, et al. Current controversies in the management of patients with severe traumatic brain injury. ANZ I Surg 2006;76:163-74.

255 Magnoni S, Ghisoni L, Locatelli M, et al. Lack of improvement in cerebral metabolism after hyperoxia in severe head injury: a microdialysis study. J Neurosurg 2003;98:952-8.

256 Longhi L, Stocchetti N. Hyperoxia in head injury: therapeutic tool? Curr Opin Crit Care 2004;10:105-9.

257 Diringer MN, Aiyagari V, Zazulia AR, et al. Effect of hyperoxia on cerebral metabolic rate for oxygen measured using positron emission tomography in patients with acute severe head injury. J Neurosurg 2007;106:526-9.

258 Davis DP, Meade W, Sise MJ, et al. Both hypoxemia and extreme hyperoxemia may be detrimental in patients with severe traumatic brain injury. I Neurotrauma 2009:26:2217-23.

259 Asher SR, Curry P, Sharma D, et al. Survival advantage and PaO2 threshold in severe traumatic brain injury. J Neurosurg Anesthesiol 2013;25:168-73.

260 Brenner M, Stein D, Hu P, et al. Association between early hyperoxia and worse outcomes after traumatic brain injury. Arch Surg 2012;147:1042-6.

261 Rincon F, Kang J, Vibbert M, et al. Significance of arterial hyperoxia and relationship with case fatality in traumatic brain injury: a multicentre cohort study. J Neurol Neurosurg Psychiatr 2014;85:799-805.

262 Kao LW, Nañagas KA. Toxicity associated with carbon monoxide. Clin Lab Med 2006;26:99-125.

263 Buckley NA, Juurlink DN, Isbister $G$, et al. Hyperbaric oxygen for carbon monoxide poisoning. Cochrane Database Syst Rev 2011;(4):CD002041.

264 Weaver LK, Valentine KJ, Hopkins RO. Carbon monoxide poisoning: risk factors for cognitive sequelae and the role of hyperbaric oxygen. Am J Respir Crit Care Med 2007;176:491-7.

265 Ford DJ, Rothwell RP. 'Safe oxygen' in acute asthma: prospective trial using 35\% Ventimask prior to admission. Respir Med 1989;83:189-94.

266 Rodrigo GJ, Rodriquez Verde M, Peregalli V, et al. Effects of short-term $28 \%$ and $100 \%$ oxygen on $\mathrm{PaCO} 2$ and peak expiratory flow rate in acute asthma: a randomized trial. Chest 2003;124:1312-17.

267 Chien JW, Ciufo R, Novak R, et al. Uncontrolled oxygen administration and respiratory failure in acute asthma. Chest 2000;117:728-33.

268 Lim WS, Baudouin SV, George RC, et al., Pneumonia Guidelines Committee of the BTS Standards of Care Committee. BTS guidelines for the management of community acquired pneumonia in adults: update 2009. Thorax 2009;64(Suppl III): $1-55$.

269 Dudgeon DJ, Lertzman M. Dyspnea in the advanced cancer patient. J Pain Symptom Manage 1998;16:212-19.

270 Ripamonti C. Management of dyspnea in advanced cancer patients. Support Care Cancer 1999;7:233-43.

271 Northfield TC. Oxygen therapy for spontaneous pneumothorax. BMJ 1971;4:86-8. 
272 Shih $\mathrm{CH}$, Tsai WH, Huang SW, et al. "Effects of high concentration oxygen treatment on traumatic pneumothorax in adult rabbits". Chin J Physiol 2012;55:178-83.

273 MacDuff A, Arnold A, Harvey J, BTS Pleural Disease Guideline Group. Management of spontaneous pneumothorax: British Thoracic Society pleural disease guideline 2010. Thorax 2010;65(Suppl 2):8-31.

274 Jardin F, Gurdjian F, Desfonds P, et al. Hemodynamic factors influencing arterial hypoxemia in massive pulmonary embolism with circulatory failure. Circulation 1979;59:909-12.

275 Creamer JE, Edwards JD, Nightingale P. Hemodynamic and oxygen transport variables in cardiogenic shock secondary to acute myocardial infarction, and response to treatment. Am J Cardiol 1990:65:1297-300.

276 Aubier M, Trippenbach T, Roussos C. Respiratory muscle fatigue during cardiogenic shock. J Appl Physiol Respir Environ Exerc Physiol 1981;51:499-508.

277 Miñana G, Núñez J, Bañuls $P$, et al. Prognostic implications of arterial blood gases in acute decompensated heart failure. Eur J Intern Med 2011;22: 489-94.

278 McMurray JJ, Adamopoulos S, Anker SD, et al., ESC Committee for Practice Guidelines. ESC guidelines for the diagnosis and treatment of acute and chronic heart failure 2012: the Task Force for the Diagnosis and Treatment of Acute and Chronic Heart Failure 2012 of the European Society of Cardiology. Developed in collaboration with the Heart Failure Association (HFA) of the ESC. Eur Heart J 2012;33:1787-847

279 Retter A, Wyncoll D, Pearse R, et al. Guidelines on the management of anaemia and red cell transfusion in adult critically ill patients. $\mathrm{Br} J$ Haematol 2013;160:445-64

280 Robieux IC, Kellner JD, Coppes MJ, et al. Analgesia in children with sickle cell crisis: comparison of intermittent opioids vs. continuous intravenous infusion of morphine and placebo-controlled study of oxygen inhalation. Pediatr Hematol Oncol 1992;9:317-26.

281 Zipursky A, Robieux IC, Brown EJ, et al. Oxygen therapy in sickle cell disease. Am J Pediatr Hematol Oncol 1992;14:222-8.

282 Homi J, Levee L, Higgs D, et al. Pulse oximetry in a cohort study of sickle cell disease. Clin Lab Haematol 1997;19:17-22.

283 Rees DC, Olujohungbe AD, Parker NE, et al. Guidelines for the management of the acute painful crisis in sickle cell disease. Br J Haematol 2003;120:744-52.

284 Centre for Clinical Practice at NICE (UK). Sickle Cell Acute Painful Episode: Management of an Acute Painful Sickle Cell Episode in Hospital. Manchester, UK: National Institute for Health and Clinical Excellence, Jun 2012.

285 Jeffrey AA, Warren PM, Flenley DC. Acute hypercapnic respiratory failure in patients with chronic obstructive lung disease: risk factors and use of guidelines for management. Thorax 1992;47:34-40.

286 Gomersall CD, Joynt GM, Freebairn RC, et al. Oxygen therapy for hypercapnic patients with chronic obstructive pulmonary disease and acute respiratory failure: a randomized, controlled pilot study. Crit Care Med 2002;30:113-16.

287 Esteban A, Cerda E, De La Cal MA, et al. Hemodynamic effects of oxygen therapy in patients with acute exacerbations of chronic obstructive pulmonary disease. Chest 1993;104:471-5.

288 Schumaker GL, Epstein SK. Managing acute respiratory failure during exacerbation of chronic obstructive pulmonary disease. Respir Care 2004;49:766-82.

289 Warren PM, Flenley DC, Millar JS, et al. Respiratory failure revisited: acute exacerbations of chronic bronchitis between 1961-68 and 1970-76. Lancet 1980;1:467-70.

290 Hutchison DC, Flenley DC, Donald KW. Controlled oxygen therapy in respiratory failure. BMJ 1964;2:1159-66.

291 Mithoefer JC, Karetzky MS, Mead GD. Oxygen therapy in respiratory failure. N Engl J Med 1967;277:947-9.

292 Schiff MM, Massaro D. Effect of oxygen administration by a Venturi apparatus on arterial blood gas values in patients with respiratory failure. $N$ Engl J Med 1967;277:950-3.

293 King TK, Ali N, Briscoe WA. Treatment of hypoxia with 24 percent oxygen. A new approach to the interpretation of data collected in a pulmonary intensive care unit. Am Rev Respir Dis 1973;108:19-29.

294 Rudolf M, Banks RA, Semple SJ. Hypercapnia during oxygen therapy in acute exacerbations of chronic respiratory failure. Hypothesis revisited. Lancet 1977;2:483-6.

295 McNicol MW, Campbell EJ. Severity of respiratory failure. Arterial blood-gases in untreated patients. Lancet 1965;1:336-8.

296 Smith JP, Stone RW, Muschenheim C. Acute respiratory failure in chronic lung disease. Observations on controlled oxygen therapy. Am Rev Respir Dis 1968;97:791-803.

297 Campbell EJ. Respiratory failure: the relation between oxygen concentrations of inspired air and arterial blood. Lancet 1960;2:10-11.

298 Campbell EJ. A method of controlled oxygen administration which reduces the risk of carbon-dioxide retention. Lancet 1960;2:12-14.

299 Davidson AC, Banham S, Elliott M, et al., BTS Standards of Care Committee Member, British Thoracic Society/Intensive Care Society Acute Hypercapnic Respiratory Failure Guideline Development Group, On behalf of the British Thoracic
Society Standards of Care Committee. BTS/ICS guideline for the ventilatory management of acute hypercapnic respiratory failure. Thorax 2016;71(Suppl 2): ii1-35.

300 Williams TA, Finn J, Fatovich D, et al. Paramedic differentiation of asthma and COPD in the prehospital setting is difficult. Prehosp Emerg Care 2015;19:535-43.

301 Lal S. Blood gases in respiratory failure. State on admission to hospital and management. Lancet 1965;1:339-41.

302 Madden BP, Kariyawasam H, Siddiqi AJ, et al. Noninvasive ventilation in cystic fibrosis patients with acute or chronic respiratory failure. Eur Respir J 2002;19:310-13.

303 Jones HA, Turner SL, Hughes JM. Performance of the large-reservoir oxygen mask (Ventimask). Lancet 1984;1:1427-31.

304 NICE. Motor neuron disease: assessment and management. 2016.

305 Quint JK, Ward L, Davison AG. Previously undiagnosed obesity hypoventilation syndrome. Thorax 2007;62:462-3.

306 Nowbar S, Burkart KM, Gonzales R, et al. Obesity-associated hypoventilation in hospitalized patients: prevalence, effects, and outcome. Am J Med 2004;116:1-7.

307 McNulty PH, King N, Scott S, et al. Effects of supplemental oxygen administration on coronary blood flow in patients undergoing cardiac catheterization. $\mathrm{Am}$ J Physiol Heart Circ Physiol 2005;288:H1057-62.

308 Harten JM, Anderson KJ, Kinsella J, et al. Normobaric hyperoxia reduces cardiac index in patients after coronary artery bypass surgery. J Cardiothorac Vasc Anesth 2005; 19:173-5

309 Frøbert 0 , Moesgaard J, Toft E, et al. Influence of oxygen tension on myocardia performance. Evaluation by tissue Doppler imaging. Cardiovasc Ultrasound 2004;2:22.

310 Maroko PR, Radvany P, Braunwald E, et al. Reduction of infarct size by oxygen inhalation following acute coronary occlusion. Circulation 1975;52:360-8.

311 Madias JE, Hood WB Jr. Reduction of precordial ST-segment elevation in patients with anterior myocardial infarction by oxygen breathing. Circulation 1976;53(3 Suppl):1198-200.

312 Fillmore SJ, Shapiro M, Killip T. Arterial oxygen tension in acute myocardial infarction. Serial analysis of clinical state and blood gas changes. Am Heart J 1970;79:620-9.

313 Waring WS, Thomson AJ, Adwani SH, et al. Cardiovascular effects of acute oxygen administration in healthy adults. J Cardiovasc Pharmacol 2003;42:245-50.

314 Kaneda T, Ku K, Inoue T, et al. Postischemic reperfusion injury can be attenuated by oxygen tension control. Jpn Circ J 2001;65:213-18.

315 Haque WA, Boehmer J, Clemson BS, et al. Hemodynamic effects of supplemental oxygen administration in congestive heart failure. J Am Coll Cardiol 1996;27:353-7.

316 McNulty PH, Robertson BJ, Tulli MA, et al. Effect of hyperoxia and vitamin C on coronary blood flow in patients with ischemic heart disease. J App/ Physiol 2007; 102:2040-5.

317 Bodetoft $\mathrm{S}$, Carlsson $\mathrm{M}$, Arheden $\mathrm{H}$, et al. Effects of oxygen inhalation on cardiac output, coronary blood flow and oxygen delivery in healthy individuals, assessed with MRI. Eur J Emerg Med 2011;18:25-30.

318 Ranchord AM, Perrin K, Weatherall M, et al. A randomised controlled trial of the effect of high concentration oxygen on myocardial ischaemia during exercise. Int J Cardiol 2012;160:201-5.

319 Rawles JM, Kenmure AC. Controlled trial of oxygen in uncomplicated myocardial infarction. BMJ 1976;1:1121-3.

320 Ranchord AM, Argyle R, Beynon R, et al. High-concentration versus titrated oxygen therapy in ST-elevation myocardial infarction: a pilot randomized controlled trial. Am Heart J 2012;163:168-75.

321 Ukholkina GB, Kostianov I, Kuchkina NV, et al. Oxygen therapy in combination with endovascular reperfusion during the first hours of acute myocardial infarction: clinical laboratory findings. Int J Intervent Cardioangiol 2005;9:45-51.

322 Nicholson C. A systematic review of the effectiveness of oxygen in reducing acute myocardial ischaemia. J Clin Nurs 2004;13:996-1007.

323 Beasley $R$, Aldington $S$, Weatherall $M$, et al. Oxygen therapy in myocardial infarction: an historical perspective. J $R$ Soc Med 2007;100:130-3.

324 Burls A, Cabello JB, Emparanza Jl, et al. Oxygen therapy for acute myocardial infarction: a systematic review and meta-analysis. Emerg Med J 2011;28:917-23.

325 Wijesinghe M, Perrin K, Ranchord A, et al. Routine use of oxygen in the treatment of myocardial infarction: systematic review. Heart 2009;95:198-202.

326 Neill WA. Effects of arterial hypoxemia and hyperoxia on oxygen availability for myocardial metabolism. Patients with and without coronary heart disease. Am J Cardiol 1969:24:166-71.

327 NICE. NICE guidelines for chest pain of recent onset. London: NICE, 2010.

328 Steg PG, James SK, Atar D, et al., Task Force on the management of ST-segment elevation acute myocardial infarction of the European Society of Cardiology (ESC). ESC Guidelines for the management of acute myocardial infarction in patients presenting with ST-segment elevation. Eur Heart J 2012;33:2569-619.

329 Anderson JL, Adams CD, Antman EM, et al. 2011 ACCF/AHA focused update incorporated into the ACC/AHA 2007 guidelines for the management of patients with unstable angina/non-ST-elevation myocardial infarction: a report of the American College of Cardiology Foundation/American Heart Association Task Force on Practice Guidelines. Circulation 2011;123:e426-579. 
330 Stub D, Smith K, Bernard S, et al. A randomized controlled trial of oxygen therapy in acute myocardial infarction Air Verses Oxygen In myocarDial infarction study (AVOID Study). Am Heart J 2012;163:339-45.e1.

331 Stub D, Smith K, Bernard S, et al. Air versus oxygen in ST-segment-elevation myocardial infarction. Circulation 2015;131:2143-50.

332 Nehme Z, Stub D, Bernard S, et al. Effect of supplemental oxygen exposure on myocardial injury in ST-elevation myocardial infarction. Heart 2016;102:444-51.

333 Institutet K. An Efficacy and Outcome Study of Supplemental Oxygen Treatment in Patients With Suspected Myocardial Infarction (DETO2X-AMI). Secondary An Efficacy and Outcome Study of Supplemental Oxygen Treatment in Patients With Suspected Myocardial Infarction (DETO2X-AMI). 2013. http://www.clinicaltrials.gov/ ct2/show/NCT01787110?term=ami+oxygen\&rank=1

334 JRCALC. UK ambulance service clinical practice guideline 2006. JRCALC, 2006.

335 Ronning OM, Guldvog B. Should stroke victims routinely receive supplemental oxygen? A quasi-randomized controlled trial. Stroke 1999;30:2033-7.

336 Roffe C, Ali K, Warusevitane A, et al. The SOS pilot study: a RCT of routine oxygen supplementation early after acute stroke-effect on recovery of neurological function at one week. PLOS ONE 2011;6:e19113.

337 Ali K, Warusevitane A, Lally F, et al. The stroke oxygen pilot study: a randomized controlled trial of the effects of routine oxygen supplementation early after acute stroke-effect on key outcomes at six months. PLOS ONE 2013;8:e59274.

338 Stroke Oxygen Study. Secondary Stroke Oxygen Study [Website]. 2014. http:/l www.so2s.co.uk/

339 Padma MV, Bhasin A, Bhatia R, et al. Normobaric oxygen therapy in acute ischemic stroke: a pilot study in Indian patients. Ann Indian Acad Neurol 2010;13:284-8.

340 Normobaric Oxygen Therapy in Acute Ischemic Stroke Trial. Secondary Normobaric Oxygen Therapy in Acute Ischemic Stroke Trial 2013. http://clinicaltrials.gov/show/ NCT00414726

341 Rincon F, Kang J, Maltenfort M, et al. Association between hyperoxia and mortality after stroke: a multicenter cohort study. Crit Care Med 2014;42:387-96.

342 Rowat AM, Dennis MS, Wardlaw JM. Hypoxaemia in acute stroke is frequent and worsens outcome. Cerebrovasc Dis 2006;21:166-72.

343 Bravata DM, Wells CK, Lo AC, et al. Processes of care associated with acute stroke outcomes. Arch Intern Med 2010;170:804-10.

344 Roffe C, Sills S, Halim M, et al. Unexpected nocturnal hypoxia in patients with acute stroke. Stroke 2003:34:2641-5.

345 Ali $K$, Sills S, Roffe $C$. The effect of different doses of oxygen administration on oxygen saturation in patients with stroke. Neurocrit Care 2005;3:24-6.

346 Chiu EHH, Liu CS, Tan TY, et al. Venturi mask adjuvant oxygen therapy in severe acute ischemic stroke. Arch Neurol 2006;63:741-4.

347 Saisch SG, Wessely S, Gardner WN. Patients with acute hyperventilation presenting to an inner-city emergency department. Chest 1996;110:952-7.

348 Steurer J, Hoffmann U, Dür $\mathrm{P}$, et al. Changes in arterial and transcutaneous oxygen and carbon dioxide tensions during and after voluntary hyperventilation. Respiration 1997;64:200-5.

349 Callaham M. Hypoxic hazards of traditional paper bag rebreathing in hyperventilating patients. Ann Emerg Med 1989;18:622-8.

350 Demeere JL. Paraquat toxicity. The use of hypoxic ventilation. Acta Anaesthesiol Belg 1984;35:219-30.

351 May A, Leone M, Afra J, et al. EFNS guidelines on the treatment of cluster headache and other trigeminal-autonomic cephalalgias. Eur J Neurol 2006;13:1066-77

352 Fogan L. Treatment of cluster headache. A double-blind comparison of oxygen v air inhalation. Arch Neurol 1985;42:362-3.

353 NICE. Headaches in over 12s: diagnosis and management. London: NICE, 2012.

354 Cohen AS, Burns B, Goadsby PJ. High-flow oxygen for treatment of cluster headache: a randomized trial. JAMA 2009;302:2451-7.

355 Ozkurt B, Cinar O, Cevik E, et al. Efficacy of high-flow oxygen therapy in all types of headache: a prospective, randomized, placebo-controlled trial. Am J Emerg Med 2012;30:1760-4.

356 Simpson KR. Intrauterine resuscitation during labor: should maternal oxygen administration be a first-line measure? Semin Fetal Neonatal Med 2008;13:362-7.

357 Fawole B, Hofmeyr GJ. Maternal oxygen administration for fetal distress. Cochrane Database Syst Rev 2012;12:CD000136.

358 Nesterenko TH, Acun C, Mohamed MA, et al. Is it a safe practice to administer oxygen during uncomplicated delivery: a randomized controlled trial? Early Hum Dev 2012:88:677-81.

359 Hamel MS, Anderson BL, Rouse DJ. Oxygen for intrauterine resuscitation: of unproved benefit and potentially harmful. Am J Obstet Gynecol 2014;211:124-7.

360 Chatmongkolchart S, Prathep S. Supplemental oxygen for caesarean section during regional anaesthesia. Cochrane Database Syst Rev 2013;6:CD006161.

361 Güngördük K, Asicioğlu O, Yildirim G, et al. Is post-partum oxygen inhalation useful for reducing vaginal blood loss during the third and fourth stages of labour? A randomised controlled study. Aust N Z J Obstet Gynaecol 2011;51:441-5.

362 Aldren CP, Barr LC, Leach RD. Hypoxaemia and postoperative pulmonary complications. Br J Surg 1991;78:1307-8.
363 Conway CM, Payne JP. Hypoxaemia associated with anaesthesia and controlled respiration. Lancet 1964;1:12-4.

364 Moller JT, Wittrup M, Johansen SH. Hypoxemia in the postanesthesia care unit: an observer study. Anesthesiology 1990;73:890-5.

365 Canet J, Ricos M, Vidal F. Early postoperative arterial oxygen desaturation. Determining factors and response to oxygen therapy. Anesth Analg 1989;69:207-12.

366 Gift AG, Stanik J, Karpenick J, et al. Oxygen saturation in postoperative patients at low risk for hypoxemia: is oxygen therapy needed? Anesth Analg 1995:80:368-72.

367 Parke R, McGuinness S, Dixon R, et al. Open-label, phase II study of routine high-flow nasal oxygen therapy in cardiac surgical patients. Br J Anaesth 2013;111:925-31.

368 Martin DS, Grocott MP III. Oxygen therapy in anaesthesia: the yin and yang of 02 Br J Anaesth 2013;111:867-71.

369 Moller JT, Johannessen NW, Espersen K, et al. Randomized evaluation of pulse oximetry in 20,802 patients: II. Perioperative events and postoperative complications. Anesthesiology 1993;78:445-53.

370 SIGN. Postoperative management in adults: guideline no 77. Edinburgh: SIGN, 2004.

371 Strachan L, Noble DW. Hypoxia and surgical patients - prevention and treatment of an unnecessary cause of morbidity and mortality. J $R$ Coll Surg Edinb 2001;46:297-302.

372 Rothen HU. Oxygen: avoid too much of a good thing! Eur J Anaesthesiol 2010;27:493-4.

373 Cashman JN, Dolin SJ. Respiratory and haemodynamic effects of acute postoperative pain management: evidence from published data. $\mathrm{Br} J$ Anaesth 2004;93:212-23.

374 Walder B, Schafer M, Henzi I, et al. Efficacy and safety of patient-controlled opioid analgesia for acute postoperative pain. A quantitative systematic review. Acta Anaesthesiol Scand 2001;45:795-804.

375 Kopka A, Wallace E, Reilly G, et al. Observational study of perioperative PtcCO2 and $\mathrm{SpO} 2$ in non-ventilated patients receiving epidural infusion or patient-controlled analgesia using a single earlobe monitor (TOSCA). $\mathrm{Br} J$ Anaesth 2007;99:567-71.

376 Al-Niaimi A, Safdar N. Supplemental perioperative oxygen for reducing surgical site infection: a meta-analysis. J Eval Clin Pract 2009;15:360-5.

377 Qadan M, Akça O, Mahid SS, et al. Perioperative supplemental oxygen therapy and surgical site infection: a meta-analysis of randomized controlled trials. Arch Surg 2009;144:359-66; discussion 66-7.

378 Gardella C, Goltra LB, Laschansky E, et al. High-concentration supplemental perioperative oxygen to reduce the incidence of postcesarean surgical site infection: a randomized controlled trial.[Erratum appears in Obstet Gynecol. 2008 Dec;112(6):1392]. Obstet Gynecol 2008;112:545-52.

379 Meyhoff CS, Wetterslev J, Jorgensen LN, et al. Effect of high perioperative oxygen fraction on surgical site infection and pulmonary complications after abdominal surgery: the PROXI randomized clinical trial. JAMA 2009;302:1543-50.

380 Bickel A, Gurevits M, Vamos R, et al. Perioperative hyperoxygenation and wound site infection following surgery for acute appendicitis: a randomized, prospective, controlled trial.[Erratum appears in Arch Surg. 2011 Aug;146(8):993 Note: Dosage error in article text]. Arch Surg 2011;146:464-70.

381 Togioka B, Galvagno S, Sumida S, et al. The role of perioperative high inspired oxygen therapy in reducing surgical site infection: a meta-analysis. Anesth Analg 2012;114:334-42.

382 Hovaguimian F, Lysakowski C, Elia N, et al. Effect of intraoperative high inspired oxygen fraction on surgical site infection, postoperative nausea and vomiting, and pulmonary function: systematic review and meta-analysis of randomized controlled trials. Anesthesiology 2013;119:303-16.

383 Meyhoff CS, Jorgensen LN, Wetterslev J, et al. Increased long-term mortality after a high perioperative inspiratory oxygen fraction during abdominal surgery: follow-up of a randomized clinical trial. Anesth Analg 2012;115:849-54.

384 Kurz A, Fleischmann E, Sessler DI, et al. Effects of supplemental oxygen and dexamethasone on surgical site infection: a factorial randomized trial. $\mathrm{Br} J$ Anaesth 2015:115:434-43.

385 Orhan-Sungur M, Kranke $P$, Sessler D, et al. Does supplemental oxygen reduce postoperative nausea and vomiting? A meta-analysis of randomized controlled trials. Anesth Analg 2008;106:1733-8.

386 Azevedo LA, Parra RS, Da Rocha JJ, et al. Hyperbaric oxygen on the healing of ischemic colonic anastomosis - an experimental study in rats. Undersea Hyperb Med 2010;37:405-11.

387 Gorur R, Hahoglu A, Uzun G, et al. Effects of hyperbaric oxygen therapy on wound healing after tracheal resection and end-to-end anastomoses in rats: results of early observations. Thorac Cardiovasc Surg 2008;56:359-62.

388 Schietroma M, Carlei F, Cecilia EM, et al. Colorectal Infraperitoneal anastomosis: the effects of perioperative supplemental oxygen administration on the anastomotic dehiscence. J Gastrointest Surg 2012;16:427-34.

389 Schietroma M, Cecilia EM, Carlei F, et al. Prevention of anastomotic leakage after total gastrectomy with perioperative supplemental oxygen administration: a 
prospective randomized, double-blind, controlled, single-center trial. Ann Surg Oncol 2013;20:1584-90.

390 Skelly A. Analgesia and sedation. In: Watkinson A, Adam A, eds. Interventional radiology. Oxford: Radcliffe Medical Press, 1996:3-11.

391 Milman N, Faurschou P, Grode G, et al. Pulse oximetry during fibreoptic bronchoscopy in local anaesthesia: frequency of hypoxaemia and effect of oxygen supplementation. Respiration 1994;61:342-7.

392 Katz AS, Michelson EL, Stawicki J, et al. Cardiac arrhythmias. Frequency during fiberoptic bronchoscopy and correlation with hypoxemia. Arch Intern Med 1981;141:603-6.

393 Meghjee SP, Marshall M, Redfern EJ, et al. Influence of patient posture on oxygen saturation during fibre-optic bronchoscopy. Respir Med 2001;95:5-8.

394 Payne CB Jr, Goyal PC, Gupta SC. Effects of transoral and transnasal fiberoptic bronchoscopy on oxygenation and cardiac rhythm. Endoscopy 1986;18:1-3.

395 Lundgren R, Häggmark S, Reiz S. Hemodynamic effects of flexible fiberoptic bronchoscopy performed under topical anesthesia. Chest 1982;82:295-9.

396 Arai T, Hatano Y, Komatsu K, et al. Real-time analysis of the change in arterial oxygen tension during endotracheal suction with a fiberoptic bronchoscope. Crit Care Med 1985;13:855-8.

397 Pirożyński M, Sliwiński P, Radwan L, et al. Bronchoalveolar lavage: comparison of three commonly used procedures. Respiration 1991;58:72-6.

398 van Zwam JP, Kapteijns EF, Lahey S, et al. Flexible bronchoscopy in supine or sitting position: a randomized prospective analysis of safety and patient comfort. J Bronchol Interv Pulmonol 2010;17:29-32.

399 Shrader DL, Lakshminarayan S. The effect of fiberoptic bronchoscopy on cardiac rhythm. Chest 1978;73:821-4.

400 Jones AM, O'Driscoll R. Do all patients require supplemental oxygen during flexible bronchoscopy? Chest 2001;119:1906-9.

401 Attaran D, Towhidi M, Amini M, et al. The relationship between peak expiratory flow rate before bronchoscopy and arterial oxygen desaturation during bronchoscopy. Acta Med Iran 2008:46:95-8.

402 Du Rand IA, Blaikley J, Booton R, et al. British Thoracic Society guideline for diagnostic flexible bronchoscopy in adults: accredited by NICE. Thorax 2013;68 (Suppl 1):i1-i44.

403 Block R, Jankowski J, Johnston D, et al. The administration of supplementary oxygen to prevent hypoxia during upper alimentary endoscopy. Endoscopy 1993;25:269-73.

404 Bell GD, Quine A, Antrobus JH, et al. Upper gastrointestinal endoscopy: a prospective randomized study comparing continuous supplemental oxygen via the nasal or oral route. Gastrointest Endosc 1992:38:319-25.

405 Bowling TE, Hadjiminas CL, Polson RJ, et al. Effects of supplemental oxygen on cardiac rhythm during upper gastrointestinal endoscopy: a randomised controlled double blind trial. Gut 1993:34:1492-7.

406 Crantock L, Cowen AE, Ward M, et al. Supplemental low flow oxygen prevents hypoxia during endoscopic cholangiopancreatography. Gastrointest Endosc 1992;38:418-20.

407 Yen D, Hu SC, Chen LS, et al. Arterial oxygen desaturation during emergent nonsedated upper gastrointestinal endoscopy in the emergency department. Am J Emerg Med 1997;15:644-7.

408 Jurell KR, O'Connor KW, Slack J, et al. Effect of supplemental oxygen on cardiopulmonary changes during gastrointestinal endoscopy. Gastrointest EndosC 1994; 40:665-70.

409 Reshef $R$, Shiller $M$, Kinberg $R$, et al. A prospective study evaluating the usefulness of continuous supplemental oxygen in various endoscopic procedures. Isr J Med Sci 1996;32:736-40

410 Thompson AM, Park KG, Kerr F, et al. Safety of fibreoptic endoscopy: analysis of cardiorespiratory events. Br J Surg 1992;79:1046-9.

411 Fisher L, Fisher A, Thomson A. Cardiopulmonary complications of ERCP in older patients. Gastrointest Endosc 2006;63:948-55.

412 Fujimura M, Ishiura $\mathrm{Y}$, Myou S, et al. Cardiopulmonary complications during gastroscopy in patients with chronic respiratory failure undergoing long-term home oxygen therapy. Endoscopy 2000;32:33-6.

413 Amar D, Greenberg MA, Menegus MA, et al. Should all patients undergoing cardiac catheterization or percutaneous transluminal coronary angioplasty receive oxygen? Chest 1994;105:727-32.

414 Deitch K, Chudnofsky CR, Dominici P. The utility of supplemental oxygen during emergency department procedural sedation and analgesia with midazolam and fentanyl: a randomized, controlled trial. Ann Emerg Med 2007;49:1-8.

415 Deitch K, Chudnofsky CR, Dominici P. The utility of supplemental oxygen during emergency department procedural sedation with propofol: a randomized, controlled trial. Ann Emerg Med 2008;52:1-8.

416 Deitch K, Miner J, Chudnofsky CR, et al. Does end tidal CO2 monitoring during emergency department procedural sedation and analgesia with propofol decrease the incidence of hypoxic events? A randomized, controlled trial. Ann Emerg Med 2010;55:258-64.

417 Friedrich-Rust M, Welte M, Welte C, et al. Capnographic monitoring of propofol-based sedation during colonoscopy. Endoscopy 2014;46:236-44.
418 Booth S, Wade R, Johnson M, et al., Expert Working Group of the Scientific Committee of the Association of Palliative Medicine. The use of oxygen in the palliation of breathlessness. A report of the expert working group of the Scientific Committee of the Association of Palliative Medicine. Respir Med 2004;98:66-77

419 Booth S, Kelly MJ, Cox NP, et al. Does oxygen help dyspnea in patients with cancer? Am J Respir Crit Care Med 1996;153:1515-18.

420 Bruera $E$, de Stoutz N, Velasco-Leiva $A$, et al. Effects of oxygen on dyspnoea in hypoxaemic terminal-cancer patients. Lancet 1993;342:13-14.

421 Currow DC, Agar M, Smith J, et al. Does palliative home oxygen improve dyspnoea? A consecutive cohort study. Palliat Med 2009;23:309-16.

422 Cranston JM, Crockett A, Currow D. Oxygen therapy for dyspnoea in adults. Cochrane Database Syst Rev 2008;(3):CD004769.

423 Bajwah S, Ross JR, Peacock JL, et al. Interventions to improve symptoms and quality of life of patients with fibrotic interstitial lung disease: a systematic review of the literature. Thorax 2013;68:867-79.

424 Uronis HE, Currow DC, McCrory DC, et al. Oxygen for relief of dyspnoea in mildlyor non-hypoxaemic patients with cancer: a systematic review and meta-analysis. $\mathrm{Br}$ J Cancer 2008:98:294-9.

425 Galbraith S, Fagan P, Perkins P, et al. Does the use of a handheld fan improve chronic dyspnea? A randomized, controlled, crossover trial. J Pain Symptom Manage 2010;39:831-8

426 Clemens KE, Quednau I, Klaschik E. Use of oxygen and opioids in the palliation of dyspnoea in hypoxic and non-hypoxic palliative care patients: a prospective study. Support Care Cancer 2009;17:367-77.

427 Ben-Aharon I, Gafter-Gvili A, Leibovici L, et al. Interventions for alleviating cancer-related dyspnea: a systematic review and meta-analysis. Acta Oncol 2012:51:996-1008.

428 Campbell ML, Yarandi H, Dove-Medows E. Oxygen is nonbeneficial for most patients who are near death. J Pain Symptom Manage 2013;45:517-23.

429 Berkenbosch JW, Grueber RE, Graff GR, et al. Patterns of helium-oxygen (heliox) usage in the critical care environment. J Intensive Care Med 2004:19:335-44.

430 Rodrigo GJ, Rodrigo C, Pollack CV, et al. Use of helium-oxygen mixtures in the treatment of acute asthma: a systematic review. Chest 2003:123:891-6.

431 Colebourn CL, Barber V, Young JD. Use of helium-oxygen mixture in adult patients presenting with exacerbations of asthma and chronic obstructive pulmonary disease: a systematic review. Anaesthesia 2007;62:34-42.

432 Brandão DC, Britto MC, Pessoa MF, et al. Heliox and forward-leaning posture improve the efficacy of nebulized bronchodilator in acute asthma: a randomized trial. Respir Care 2011;56:947-52.

433 Gerbeaux P, Gainnier M, Boussuges A, et al. Use of heliox in patients with severe exacerbation of chronic obstructive pulmonary disease. Crit Care Med 2001:29:2322-4.

434 Andrews R, Lynch M. Heliox in the treatment of chronic obstructive pulmonary disease. Emerg Med J 2004;21:670-5.

435 Giles TL, Lasserson TJ, Smith BJ, et al. Continuous positive airways pressure for obstructive sleep apnoea in adults. Cochrane Database Syst Rev 2006;(1):CD001106.

436 Kaw R, Chung F, Pasupuleti V, et al. Meta-analysis of the association between obstructive sleep apnoea and postoperative outcome. $\mathrm{Br} J$ Anaesth 2012:109:897-906.

437 Squadrone V, Coha M, Cerutti E, et al., Piedmont Intensive Care Units Network (PICUN). Continuous positive airway pressure for treatment of postoperative hypoxemia: a randomized controlled trial. JAMA 2005;293:589-95.

438 Rennotte MT, Baele P, Aubert G, et al. Nasal continuous positive airway pressure in the perioperative management of patients with obstructive sleep apnea submitted to surgery. Chest 1995;107:367-74.

439 Society BOaMS. BOMSS Standards for Clinical Services \& Guidance on Commissioning. London: BOMSS, 2012.

440 Ahmad S, Nagle A, McCarthy RJ, et al. Postoperative hypoxemia in morbidly obese patients with and without obstructive sleep apnea undergoing laparoscopic bariatric surgery. Anesth Analg 2008;107:138-43.

441 Peter JV, Moran JL, Phillips-Hughes J, et al. Effect of non-invasive positive pressure ventilation (NIPPV) on mortality in patients with acute cardiogenic pulmonary oedema: a meta-analysis. Lancet 2006;367:1155-63.

442 Vital FM, Ladeira MT, Atallah AN. Non-invasive positive pressure ventilation (CPAP or bilevel NPPV) for cardiogenic pulmonary oedema. Cochrane Database Syst Rev 2013:5:CD005351.

443 Gray A, Goodacre S, Newby DE, et al. Noninvasive ventilation in acute cardiogenic pulmonary edema. N Engl J Med 2008;359:142-51.

444 Thompson J, Petrie DA, Ackroyd-Stolarz $S$, et al. Out-of-hospital continuous positive airway pressure ventilation versus usual care in acute respiratory failure: a randomized controlled trial. Ann Emerg Med 2008;52:232-41, 241.e1.

445 Austin M, Wood-Baker R. Oxygen therapy in the pre-hospital setting for acute exacerbations of chronic obstructive pulmonary disease. Cochrane Database Syst Rev 2006;(3):CD005534.

446 HSCIC. Hospital Episode statistics 2011/12 Admitted patient care England. Secondary Hospital Episode statistics 2011/12 Admitted patient care England. 
2012. http://www.hscic.gov.uk/article/2021/Website-Search?productid=9161\&q= hospital+episode+statistics $+2012+$ copd\&sort=Relevance $\&$ size $=10 \&$ page $=$ 1\&area=both\#top

447 Durrington HJ, Flubacher M, Ramsay CF, et al. Initial oxygen management in patients with an exacerbation of chronic obstructive pulmonary disease. QJM 2005:98:499-504

448 Gooptu B, Ward L, Ansari SO, et al. Oxygen alert cards and controlled oxygen: preventing emergency admissions at risk of hypercapnic acidosis receiving high inspired oxygen concentrations in ambulances and A\&E departments. Emerg Med J 2006;23:636-8.

449 Sprehn M, Trautner $\mathrm{S}$, Wiingaard $\mathrm{S}$, et al. [Prehospital pain relief with nitrous oxide (Entonox)]. Ugeskr Laeg 1994;156:5830-5.

450 Su F, Wei $X$, Chen $X$, et al. [Clinical study on efficacy and safety of labor analgesia with inhalation of nitrous oxide in oxygen]. Zhonghua Fu Chan Ke Za Zhi 2002;37:584-7.

451 BOC. Discover Entonox. Secondary Discover Entonox 2013. http://www.entonox.co. uk/en/discover_enotonox/index.shtml

452 Kelly FE, Hardy R, Hall EA, et al. Fire on an intensive care unit caused by an oxygen cylinder. Anaesthesia 2013;68:102-4.

453 Macdonald AG. A brief historical review of non-anaesthetic causes of fires and explosions in the operating room. Br J Anaesth 1994;73:847-56.

454 MacDonald AG. A short history of fires and explosions caused by anaesthetic agents. Br J Anaesth 1994;72:710-22.

455 Boumphrey SM, Morris EAJ, Kinsella SM. 100\% inspired oxygen from a Hudson mask-a realistic goal? Resuscitation 2003;57:69-72.

456 Milross J, Young IH, Donnelly P. The oxygen delivery characteristics of the Hudson Oxy-one face mask. Anaesth Intensive Care 1989;17:180-4.

457 Stausholm K, Rosenberg-Adamsen S, Skriver M, et al. Comparison of three devices for oxygen administration in the late postoperative period. $\mathrm{Br} J$ Anaesth 1995;74:607-9.

458 Costello RW, Liston R, McNicholas WT. Compliance at night with low flow oxygen therapy: a comparison of nasal cannulae and Venturi face masks. Thorax 1995;50:405-6.

459 Zevola DR, Maier CB. Use of nasal cannula versus face mask after extubation in patients after cardiothoracic surgery. Crit Care Nurse 2001;21:47-53.

460 Hunter J, Olson LG. Performance of the Hudson Multi-Vent oxygen mask. Med J Aust 1988:148:444-7.

461 DeGaute JP, Domenighetti G, Naeije R, et al. Oxygen delivery in acute exacerbation of chronic obstructive pulmonary disease. Effects of controlled oxygen therapy. Am Rev Respir Dis 1981;124:26-30.

462 Bone RC, Pierce AK, Johnson RL Jr. Controlled oxygen administration in acute respiratory failure in chronic obstructive pulmonary disease: a reappraisal. Am J Med 1978;65:896-902.

463 Bazuaye EA, Stone TN, Corris PA, et al. Variability of inspired oxygen concentration with nasal cannulas. Thorax 1992;47:609-11.

464 Wettstein RB, Shelledy DC, Peters JI. Delivered oxygen concentrations using low-flow and high-flow nasal cannulas. Respir Care 2005;50:604-9.

465 Agustí AG, Carrera M, Barbé $F$, et al. Oxygen therapy during exacerbations of chronic obstructive pulmonary disease. Eur Respir J 1999;14:934-9.

466 Williams $A B$, Jones PL, Mapleson WW. A comparison of oxygen therapy devices used in the postoperative recovery period. Anaesthesia 1988;43:131-5.

467 Bolton J, Russell WJ. Are nasal spectacles adequate for supplementary oxygen in patients after anaesthesia? Int J Nurs Pract 2001;7:329-35.

468 Nolan KM, Winyard JA, Goldhill DR. Comparison of nasal cannulae with face mask for oxygen administration to postoperative patients. $\mathrm{Br} J$ Anaesth 1993;70:440-2.

469 Ritchie JE, Williams AB, Gerard C, et al. Evaluation of a humidified nasal high-flow oxygen system, using oxygraphy, capnography and measurement of upper airway pressures. Anaesth Intensive Care 2011;39:1103-10.

470 Tiruvoipati R, Lewis D, Haji K, et al. High-flow nasal oxygen vs high-flow face mask: a randomized crossover trial in extubated patients. J Crit Care 2010;25:463-8.
471 Hernández G, Vaquero C, González P, et al. Effect of postextubation high-flow nasal cannula vs conventional oxygen therapy on reintubation in low-risk patients: a randomized clinical trial. JAMA 2016;315:1354-61.

472 Roca 0 , Riera J, Torres $\mathrm{F}$, et al. High-flow oxygen therapy in acute respiratory failure. Respir Care 2010;55:408-13.

473 Cuquemelle E, Pham T, Papon JF, et al. Heated and humidified high-flow oxygen therapy reduces discomfort during hypoxemic respiratory failure. Respir Care 2012;57:1571-7.

474 Parke RL, McGuinness SP, Eccleston ML. A preliminary randomized controlled trial to assess effectiveness of nasal high-flow oxygen in intensive care patients. Respir Care 2011;56:265-70.

475 Frat JP, Thille AW, Mercat A, et al. High-flow oxygen through nasal cannula in acute hypoxemic respiratory failure. N Engl J Med 2015;372:2185-96.

476 HSE. Safe use of gas cylinders. 2004; (1). http://www.hse.gov.uk/cdg/pdf/safusgc.pdf

477 HSE. The carriage of dangerous goods and use of transportable pressure equipment regulations 2004. London: HMSO, 2004.

478 Khan SY, O'Driscoll BR. Is nebulized saline a placebo in COPD? BMC Pulm Med 2004;4:9.

479 Poole PJ, Brodie SM, Stewart JM, et al. The effects of nebulised isotonic saline and terbutaline on breathlessness in severe chronic obstructive pulmonary disease (COPD). Aust N Z J Med 1998;28:322-6.

480 Conway JH, Fleming JS, Perring $S$, et al. Humidification as an adjunct to chest physiotherapy in aiding tracheo-bronchial clearance in patients with bronchiectasis. Respir Med 1992;86:109-14.

481 Andres D, Thurston N, Brant R, et al. Randomized double-blind trial of the effects of humidified compared with nonhumidified low flow oxygen therapy on the symptoms of patients. Can Respir J 1997;4:76-80.

482 Campbell EJ, Baker MD, Crites-Silver P. Subjective effects of humidification of oxygen for delivery by nasal cannula. A prospective study. Chest 1988;93:289-93.

483 Cameron JL, Reese WA, Tayal VS, et al. Bacterial contamination of ambulance oxygen humidifier water reservoirs: a potential source of pulmonary infection. Ann Emerg Med 1986;15:1300-2.

484 Khoukaz G, Gross NJ. Effects of salmeterol on arterial blood gases in patients with stable chronic obstructive pulmonary disease. Comparison with albuterol and ipratropium. Am J Respir Crit Care Med 1999;160:1028-30.

485 Gunawardena KA, Patel B, Campbell IA, et al. Oxygen as a driving gas for nebulisers: safe or dangerous? BMJ (Clin Res Ed) 1984;288:272-4.

486 Hedges JR, Cionni DJ, Amsterdam JT, et al. Oxygen desaturation in adults following inhaled metaproterenol therapy. J Emerg Med 1987;5:77-81.

487 Higgins RM, Cookson WO, Chadwick GA. Changes in blood gas levels after nebuhaler and nebulizer administration of terbutaline in severe chronic airway obstruction. Bull Eur Physiopathol Respir 1987;23:261-4.

488 Edwards L, Perrin K, Williams M, et al. Randomised controlled crossover trial of the effect on PtCO2 of oxygen-driven versus air-driven nebulisers in severe chronic obstructive pulmonary disease. Emerg Med J 2012;29:894-8.

489 Standley TDA, Smith HL, Brennan LJ, et al. Room air dilution of heliox given by facemask. Intensive Care Med 2008;34:1469-76.

490 Teague R. Safety and sedation during endoscopic procedures: BSG guideline. London: British Society of Gastroenterology, 2003.

491 Green J. Guidelines on complications of gastrointestinal endoscopy. London: British Society for Gastroenterology, 2006.

492 Evans EN, Ganeshalingam K, Ebden P. Changes in oxygen saturation and transcutaneous carbon dioxide and oxygen levels in patients undergoing fibreoptic bronchoscopy. Respir Med 1998;92:739-42.

493 Society BT. BTS National Respiratory Audit Programme Annual Report 2012/13. Secondary BTS National Respiratory Audit Programme Annual Report 2012/13. 2013. https://www.brit-thoracic.org.uk/document-library/ audit-and-quality-improvement/audit-reports/ bts-national-respiratory-audit-programme-annual-report-2012-2013/

494 O'Driscoll BR. British Thoracic Society Emergency Oxygen Audit Report 2015. London: British Thoracic Society, 2015:8. 
Annex 1 Guideline group members, representation and guideline sections

\begin{tabular}{|c|c|c|c|c|}
\hline Name & Location & Specialty & Representing & Guideline section \\
\hline $\begin{array}{l}\text { Dr Ronan O'Driscoll } \\
\text { Co-Chair } \\
\text { Organisation and Chief Editor }\end{array}$ & Salford & General/respiratory medicine & BTS & Full and concise \\
\hline $\begin{array}{l}\text { Dr Luke Howard } \\
\text { Co-Chair } \\
\text { Academic }\end{array}$ & London & Respiratory medicine & BTS & Full and concise \\
\hline $\begin{array}{l}\text { Dr Vincent Mak } \\
\text { Co-Chair implementation }\end{array}$ & London & Respiratory medicine & BTS & Full and concise \\
\hline $\begin{array}{l}\text { Dr John Earis } \\
\text { Co-Chair } \\
\text { Education }\end{array}$ & Liverpool & Respiratory medicine/education & BTS & Full and concise \\
\hline Professor Anthony Davison & Southend & Respiratory medicine & Royal College of Physicians, London & Overview \\
\hline Professor Richard Beasley & New Zealand & Respiratory medicine & & Overview \\
\hline Dr Duncan Powrie & Southend & General/respiratory medicine & & 7 \\
\hline Dr Louise Restrick & London & Respiratory medicine & & Overview \\
\hline Dr Mervyn Singer & UCL & Intensive care medicine & & Overview \\
\hline Professor Wisia Wedzicha & London & Respiratory medicine & & Overview \\
\hline Dr Jeff Keep & London & Emergency medicine & College of Emergency Medicine & 8 \\
\hline Dr Roop Kishen & Salford & Intensive care & Intensive Care Society & 8 \\
\hline Dr Muhammed Asad Iqbal Khan & Bury & Acute medicine & Society for Acute Medicine & $3,4,5$ \\
\hline Dr Meme Wijesinghe & Truro & Respiratory consultant & & 8 \\
\hline Dr lain Small & & Primary care & PCRS UK & 10,11 \\
\hline Carol Kelly & Ormskirk & Nurse & RCN/ARNS & 8 \\
\hline Lisa Ward & Southend & Nurse & ARNS & 8,9 \\
\hline Leigh Mansfield & Plymouth & Physiotherapy & ACPRC/CSP & $3,4,5$ \\
\hline Angela Evans & North Staffs & ARTP & ARTP & 7 \\
\hline Dr Fionna Moore & London & Ambulance service & JRCALC & $3,4,5$ \\
\hline David Whitmore & London & Ambulance service & $\begin{array}{l}\text { College of Paramedics/LAS } \\
\text { Faculty of Pre-Hospital Care, RCSE }\end{array}$ & 8 \\
\hline Dr Simon Gibbs & London & Cardiology & British Cardiovascular Society & $3,4,5$ \\
\hline Dr Bruce Martin & London & Anaesthesia & Royal College of Anaesthetists & 8 \\
\hline Dr Kim Hinshaw & London & Obstetrics & Royal College of Obstetrics and Gynaecology & 8 \\
\hline Dr Jasmeet Soar & & Intensive care medicine & Resuscitation Council UK & 8 \\
\hline Professor Christine Roffe & Keele & Stroke medicine & British Association of Stroke Physicians & 8 \\
\hline Dr Robin Howard & & Neurology & Association of British Neurologists & 8 \\
\hline Dr Alistair Dorward & Glasgow & Respiratory & Royal College of Physicians of Edinburgh & 8 \\
\hline Dr Chris Dyer & & Geriatrics & British Geriatric Society & 8,9 \\
\hline Clare Fitzpatrick & & Midwifery & Royal College of Midwives & 8 \\
\hline Hasanin Khachi & Barts and the London NHS Trust & Pharmacy & Royal Pharmaceutical Society & 8 \\
\hline Dr Sabrina Bajwah & King's College Hospital & Palliative medicine & Association of Palliative Medicine & 8 \\
\hline Dr Binita Kane & Salford & ST & & 8 \\
\hline Dr Katrina Curtis & Bristol & ST & & 8 \\
\hline Lucy Falconer & & Patient representative & & Overview \\
\hline
\end{tabular}

